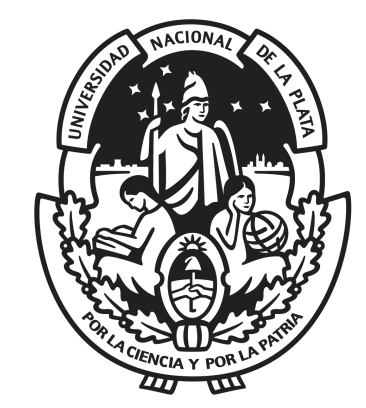

\title{
Control de sistemas de Generación Distribuida Renovable mediante conceptos de Pasividad y de moldeo de potencia.
}

\author{
Tesis de Doctorado
}

Ricardo R. Peña

\author{
Presentada ante la Facultad de Ingeniería de la \\ Universidad Nacional de La Plata \\ como requisito para la obtención del grado académico de \\ DOCTOR EN INGENIERÍA
}

Dirección de tesis:

\author{
Ing. Ricardo J. Mantz \\ Dr. Roberto D. Fernández
}

Jurado de Tesis:

Dr. Jorge Alberto Solsona

Dr. Patricio Donato

Dr. Guillermo R. Bossio

Fecha de la defensa oral y pública: 7 de julio de 2016 . 

A Silvana y a mi familia. 



\section{Agradecimientos}

Deseo expresar mi agradecimiento a:

A mis Directores, Ricardo Julian Mantz y Roberto Daniel Fernández por su paciencia, explicaciones acerca de control automático, máquinas eléctricas y consejos en función de su amplia experiencia.

A Pedro Eduardo Battaiotto por todas las explicaciones acerca de máquinas y redes eléctricas y sobre todo por su permanente entusiasmo.

A mi papá Ricardo y a mi mamá Carmen por su permanente apoyo durante todos estos años.

A Silvana Diaz, por tenerme tanta paciencia y por cada palabra de aliento para terminar la tesis.

A la Facultad de Ingeniería de la Universidad Nacional de la Patagonia y al departamento de electrónica de la UNPSJB.

A Jaqueline Taxer, Lucas Luengo y Nacho Verde por brindarme una mano en mi estadía en La Plata, su amistad y todos los asados que compartimos.

A Walter Olivera, Julia Steger, Javier Askenazi, Cristian Sosa, Nicolas Costa, Gerardo Arthz, Raul Ceballos y a mis tíos Arturo y Matilde.

Finalmente, a todos los integrantes y amigos del instituto LEICI, dependiente de la FIUNLP por brindarme una mano cada vez que necesite ayuda con algo, mates, charlas, etc. 


\section{ÍNDICE GENERAL}

$\begin{array}{lll}\text { Lista de figuras } & \text { XI }\end{array}$

1. Introducción 1

1.1. Antecedentes y motivaciones . . . . . . . . . . . . . . . 1

1.2. Organización y estructura de la tesis . . . . . . . . . . . . . 2

1.3. Principales aportes . . . . . . . . . . . . . . . . . 4

2. Recurso eólico $\quad 7$

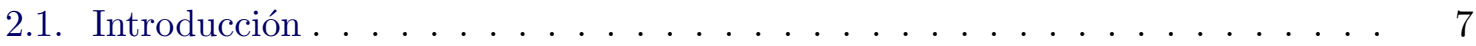

2.2. Naturaleza del viento . . . . . . . . . . . . . . . . . . . . . . 8

2.2.1. Distribución estadística . . . . . . . . . . . . . . . . . 99 9

2.2.2. Energía y potencia disponible en el viento . . . . . . . . . . . . . . 10

2.2.3. Turbulencias . . . . . . . . . . . . . . . . . . . . 10

2.3. Turbina eólica . . . . . . . . . . . . . . . . . . . . . . 10

2.3.1. Curva de potencia eléctrica de un aerogenerador . . . . . . . . . . 17

2.3.2. Modelo de la turbina eólica . . . . . . . . . . . . . . . . . . . . . . 18

2.4. Topologías de sistemas de conversión de energía eólica . . . . . . . . . . 20

2.4.1. Aerogeneradores de velocidad fija . . . . . . . . . . . . . . . . 20

2.4.2. Aerogeneradores de velocidad variable . . . . . . . . . . . . . 21

2.5. Requerimientos de red para la integración de energía eólica . . . . . . . . 23

2.5.1. Control de potencia activa y respuesta a cambios de frecuencia . . . . 25

2.5.2. Regulación de potencia reactiva y de tensión . . . . . . . . . . 25

2.5.3. Rango de frecuencias de funcionamiento . . . . . . . . . . . . 26

2.5.4. Capacidad para manejar una falla (FRT) . . . . . . . . . . . . 27

2.6. Retrospección . . . . . . . . . . . . . . . . . . . . 28

3. Redes 29

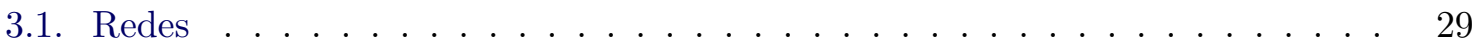

3.1.1. Líneas de transmisión de AC . . . . . . . . . . . . . 30

3.1.2. Clasificación de los diferentes tipos de buses . . . . . . . . . . . . . . 32

3.1.3. Ecuaciones de redes . . . . . . . . . . . . . . . . . . . 32

3.1.4. Redes débiles . . . . . . . . . . . . . . . . . . . . . 34

3.2. Cargas estáticas y dinámicas . . . . . . . . . . . . . 34

3.2.1. Cargas estáticas . . . . . . . . . . . . . . 35 
3.2.2. Cargas dinámicas . . . . . . . . . . . . . . . . 36

3.3. Generador sincrónico . . . . . . . . . . . . . . . . . . 37

3.4. Sistemas de almacenamiento de energía . . . . . . . . . . . . . . 40

3.4.1. Tecnologías en sistemas de almacenamiento . . . . . . . . . . . . 41

3.4.2. Sistemas de almacenamiento de energía aplicados a sistemas de generación de energía eólica . . . . . . . . . . . . . . . . . . 44

3.5. Micro red . . . . . . . . . . . . . . . . . . . . . . 45

3.5.1. Conceptos de Micro red . . . . . . . . . . . . . . . . 46

3.5.2. Configuración típica de una micro red . . . . . . . . . . . . 46

3.5.3. Sistemas de control en una micro red . . . . . . . . . . . . . . . . 48

3.5.4. Curvas de decaimiento . . . . . . . . . . . . . . . . . . 50

3.6. Retrospección . . . . . . . . . . . . . . . . . . 53

4. Control Vectorial de máquinas DARB $\mathbf{5 5}$

4.1. Introducción al control vectorial en una máquina de inducción . . . . . . . . 55

4.2. Transformación del marco de referencia . . . . . . . . . . . . . . . . . 57

4.2.1. Transformación del marco de referencia $a b c / d q \ldots \ldots . . . . . .557$

4.2.2. Transformación del marco de referencia $a b c / D Q \ldots \ldots . . . . . .59$

4.2.3. Par electromagnético en una máquina de inducción de corriente alterna 59

4.2.4. Ecuaciones fasoriales de la máquina . . . . . . . . . . . . . 63

4.2.5. Aplicación de un marco de referencia fijo al fasor de flujo estatórico . $\quad 64$

4.3. Control de la máquina de inducción de rotor bobinado (DFIG) . . . . . . 66

4.3.1. Control por flujo estatórico . . . . . . . . . . . . . 67

4.3.2. Tensión estatórica para una máquina de rotor bobinado . . . . . . . 68

4.3.3. Tensión rotórica para una máquina de rotor bobinado . . . . . . . . . . 69

4.3.4. Control vectorial orientado según flujo estatórico . . . . . . . . . . . 69

4.3.5. Control vectorial según la tensión de línea o flujo de red . . . . . . . . 71

4.4. Retrospección . . . . . . . . . . . . . . . . . . . . . 73

5. Control basado en Pasividad $\quad 75$

5.1 . Introducción . . . . . . . . . . . . . . . . . . . 75

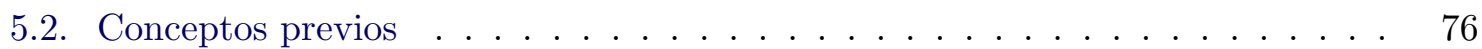

5.2.1. Disipatividad y pasividad . . . . . . . . . . . . . 77

5.2.2. Interconexión de sistemas pasivos . . . . . . . . . . . . . . 78

5.2.3. Exceso y falta de pasividad . . . . . . . . . . . . . . . 81

5.2.4. Estabilidad en el sentido de Lyapunov . . . . . . . . . . . . . . . . 82

5.2.5. Lema de Kalman-Yakubovich-Popov (KYP) . . . . . . . . . . . . . . . 84

5.2.6. Estabilidad de sistemas pasivos . . . . . . . . . . . . . . . . 85

5.3. CBP a partir del balance de energía . . . . . . . . . . . . 86

5.3.1. Balance de energía . . . . . . . . . . . . . . 87

5.4. CBP a partir del balance de potencia . . . . . . . . . . . . . . . 90

5.4.1. Modelado basado en potencia utilizando las ecuaciones de Brayton-Moser 90

5.4.2. Síntesis de un controlador empleando moldeo de potencia . . . . . . . 91

5.4.3. Representación alternativa de los pares $Q(x), P(x) \ldots \ldots \ldots 9$

5.4.4. Balance de potencia con Control Integral _ . . . . . . . . . . . . . . 95

5.5. Retrospección . . . . . . . . . . . . . . . . . 96 
6. Control por moldeo de potencia en GDR 99

6.1. Introducción . . . . . . . . . . . . . . . . . . . . . . . 99

6.2. Control de aerogeneradores con conexión a red . . . . . . . . . . . . . . 101

6.2.1. Modelo dinámico del aerogenerador . . . . . . . . . . . . . . . . . . . 102

6.2.2. Control por moldeo de potencia . . . . . . . . . . . . . . . . . . . . 104

6.2.3. Control de la potencia activa . . . . . . . . . . . . . 106

6.2.4. Controladores de potencias activa y reactiva con acción integral . . . . 111

6.2.5. Control de una granja eólica . . . . . . . . . . . . . . . . . 114

6.3. Aplicación a una micro red . . . . . . . . . . . . . . . . . 121

6.3.1. Topología base y componentes . . . . . . . . . . . . . . . . . 121

6.3.2. Ley de control a partir de moldeo de potencia . . . . . . . . . . . . 126

6.3.3. Evaluación de la ley de control . . . . . . . . . . . . . . . . . . 128

6.4. Retrospección . . . . . . . . . . . . . . . . . . . . 135

$\begin{array}{ll}\text { 7. Conclusiones } & 137\end{array}$

7.1. Evaluación de resultados . . . . . . . . . . . . . . . . . . . 137

7.2. Futuras Líneas de investigación . . . . . . . . . . . . . . . . 138

$\begin{array}{ll}\text { Referencias } & 141\end{array}$ 


\section{ÍNDICE DE FIGURAS}

2.1. Espectro de Van der Hoven. . . . . . . . . . . . . . . . . . . 8

2.2. Distribución de Weibull $k=1,2, k=2, k=3$ en líneas de trazo y punto, trazo y llena, respectivamente. . . . . . . . . . . . . . . . 9

2.3. Elementos principales de un aerogenerador. . . . . . . . . . . . . . . . 11

2.4. Turbina eólica de eje horizontal. . . . . . . . . . . . . . . . . 11

2.5. Perfil vertical de la velocidad del viento según la altura, según aproximación

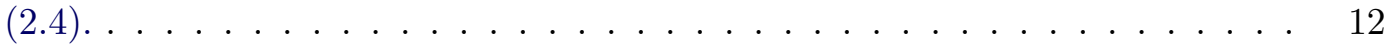

2.6. Tubo de corriente de aire. . . . . . . . . . . . . . . . . . . 13

2.7. Velocidades y fuerzas en un elemento de pala de una turbina eólica. . . . . . 14

2.8. Potencia mecánica de una turbina eólica y curva de la potencia óptima (línea azul) desarrollada. . . . . . . . . . . . . . . . . . . . 15

2.9. Par mecánico desarrollado por una turbina eólica para diferentes velocidades de viento y curva del par óptimo (línea azul). . . . . . . . . . . . . . . 16

2.10. Aproximación analítica del coeficiente de potencia $\left(C_{p}(\lambda, \beta)\right) \ldots \ldots \ldots$

2.11. Curva de potencia típica de un aerogenerador de $1,8 \mathrm{MW} \ldots \ldots \ldots \ldots$

2.12. Modelo del actuador de pitch. . . . . . . . . . . . . . . . 20

2.13. Esquema de aerogenerador de velocidad fija. . . . . . . . . . . . . 20

2.14. Esquema de aerogenerador equipado con un DFIG. . . . . . . . . . . . . 22

2.15. Esquema de un aerogenerador con un convertidor completo. . . . . . . . . . 23

2.16. Forma típica de expresar requerimientos de operación continua y reducida. . . 24

2.17. Especificaciones de control de potencia, delta y gradiente. . . . . . . . . 26

2.18. Rango de frecuencias de operación para diferentes operadores. . . . . . . . . 27

2.19. Límite para un requerimiento de FRT . . . . . . . . . . . . . . . . . 28

3.1. Diagrama equivalente del sistema de potencia. . . . . . . . . . . . . . . . . 30

3.2. Diagrama fasorial. . . . . . . . . . . . . . . . . . 30

3.3. Sistema de Control para un sistema de generación sincrónica. . . . . . . . . . 37

3.4. Esquema de un ESS directo. . . . . . . . . . . . . . . . . . . . . 42

3.5. Sistemas de almacenamiento indirecto. . . . . . . . . . . . . . . . 42

3.6. ESS, aplicados a un sistema de potencia. . . . . . . . . . . . . . . 43

3.7. Diagrama de una micro red. . . . . . . . . . . . . . . . . . . 47

3.8. Esquema básico de un sistema de generación de energía. . . . . . . . . . . 50

3.9. Curva decaimiento de Q-V. . . . . . . . . . . . . . . 50 
3.10. Curva de decaimiento de la potencia activa en función de la frecuencia para una máquina. . . . . . . . . . . . . . . . . . 51

3.11. Cálculo del ángulo $\left(\delta_{v}\right)$ a partir de la característica de decaimiento. . . . . . 52

3.12. Curvas de decaimiento de la potencia activa en función de la frecuencia. . . . 52

4.1. Fasor $\bar{x}$ y las tres variables $x_{a}, x_{b}$ y $x_{c} \ldots \ldots \ldots \ldots \ldots \ldots$

4.2. Transformación de las variables en el marco de referencia trifásico abc a un

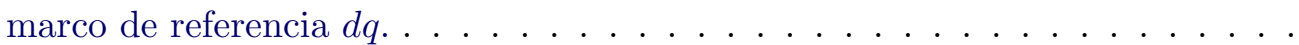

4.3. Relación entre el marco de referencia especial fijo al estator y el marco de referencia estacionario. . . . . . . . . . . . . . . .

4.4. Esquema de conexión de un generador de inducción doblemente alimentado

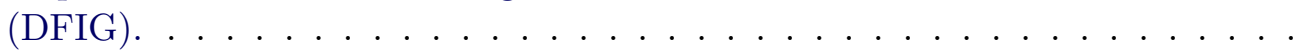

4.5. Fasor de la corriente magnetizante del estator y fasor de la corriente del rotor.

4.6. Esquema del control de una máquina de rotor bobinado (DFIG) empleando el marco de referencia según el flujo estatórico. . . . . . . . . . . . . . . .

4.7. Esquema del control de una máquina de rotor bobinado (DFIG) empleando el marco de referencia orientado por flujo de red. . . . . . . . . . . . .

5.1. Interconexión en paralelo de sistemas pasivos. . . . . . . . . . . . . . . . 79

5.2. Interconexión en realimentación de sistemas pasivos. . . . . . . . . . . . . 80

5.3. Pre y post multiplicación por una matriz dependiente de los estados. . . . . . 80

6.1. Sistema de conversión con generador asincrónico de rotor bobinado conectado a una red de distribución. . . . . . . . . . . . . . . . . . . . . . . . 102

6.2. Esquema del sistema considerado. . . . . . . . . . . . . . . . . . . 109

6.3. Curva de seguimiento del aerogenerador. . . . . . . . . . . . . . . . . . 110

6.4. Comportamiento del sistema analizado ante un escalón de viento. a) Escalón de viento. b) Potencia activa. c) Velocidad del eje del aerogenerador. . . . . . 111

6.5. Hueco de tensión. a) Tensión sobre el sistema de $120 \mathrm{kV}$ y el punto de conexión. b) Potencia activa. c) Velocidad del eje del aerogenerador. . . . . . . . . . . . 111

6.6. Esquema de la implementación de la técnica de control con acción integral para la granja eólica. . . . . . . . . . . . . . . . . . . . . 116

6.7. Sistema analizado frente a un escalón de viento. a) Escalón en la velocidad del viento. b) Potencia activa. c) Par mecánico. d) Velocidad en el eje. . . . . . . 117

6.8. Sistema analizado frente a un escalón de viento. a) Escalón en la velocidad del viento. b) Acción integral. c) Error en $i_{r y} ;\left(x_{2}-x_{2}^{*}\right) . \ldots$. . . . . . . . . . 117

6.9. a) Tensión en el bus de $120 \mathrm{kV}$ (línea continua y tensión en el punto de conexión utilizando CC(línea continua), SC (línea de trazos) y una estrategia PI (línea de trazos y puntos). b) Corriente inyectada por la granja eólica c) Potencia reactiva en los tres casos analizados. . . . . . . . . . . . . . .

6.10. a) Tensión en el bus de $120 \mathrm{kV}$ (línea continua) y tensión en el punto de conexión para los tres casos analizados. b) Potencia activa. c) Velocidad del eje del rotor. . . . . . . . . . . . . . . . . . .

6.11. a) Tensión en el bus de $120 k V$ (línea continua) y tensión en el punto de conexión para las dos estrategias analizadas. b) Salida del integrador. c) Error en $i_{r x} ;\left(x_{1}^{*}-x_{1}\right) \ldots \ldots \ldots \ldots \ldots \ldots \ldots \ldots$

6.12. a) Corriente de secuencia positiva (línea continua) y corriente de secuencia negativa (línea de trazos) en el punto de conexión. b) Potencia activa. c) Potencia reactiva. d) Par electromagnético. . . . . . . . . . . . . . . . . . . . 120

6.13. Esquema de la red considerada. . . . . . . . . . . . . . . . . . . 122 
6.14. Esquema de la micro red considerada. . . . . . . . . . . . . . . . . 123

6.15. Esquema de la implementación del lazo de acondicionamiento de la referencia. 128

6.16. Curvas de nivel y superficie de la función $P(x)$ (lazo abierto). . . . . . . . . 129

6.17. Curvas de nivel y superficie de la función $P_{d}(x) \ldots \ldots \ldots \ldots$. . . . . . 130

6.18. Curvas de nivel de las funciones $P(x)$ (líneas de trazos) y $P_{d}(x)$ (en líneas llenas).130

6.19. Curvas de nivel y superficie de la función $P_{d}(x)$ para dos elecciones del par

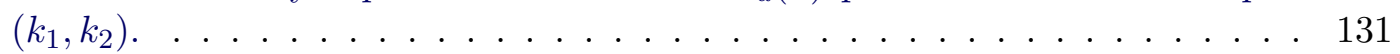

6.20. Tensión y $\delta$ en el nodo $1 . \ldots \ldots \ldots \ldots \ldots$. . . . . . . . . . . . 132

6.21. Potencia activa en la red eléctrica (en línea continua se representa cuando interviene el ESS y en línea de trazos cuando el ESS no participa). . . . . . . 133

6.22. Potencia reactiva en la red eléctrica (en línea continua se representa cuando interviene el ESS y en línea de trazos cuando el ESS no participa). . . . . . . 134 


\section{CAPÍtulo 1}

\section{INTRODUCCIÓN}

\subsection{Antecedentes y motivaciones}

Los sistemas tradicionales de generación de energía enfrentan los vaivenes del valor de los combustibles fósiles e inducen, en muchos casos, la dependencia geopolítica de los países respecto de un recurso (el combustible) que, generalmente, no les pertenece. En ese sentido, se busca la diversificación de la matriz energética y la optimización de la eficiencia de conversión tanto para maximizar cada recurso como para evitar la contaminación del medio ambiente (Chowdhury et al., 2009). De hecho, el Consejo Mundial de la Energía prevé que en el año 2050 la generación de energía eléctrica deberá ser una combinación de, al menos, ocho fuentes de generación: carbón, petróleo, gas, nuclear, hidráulica, eólica, solar, biomasa, ninguno de estos métodos deberá superar el $30 \%$ de la generación. Así, la integración de energías renovables y de la generación de electricidad existente en un marco unificado representa un reto tecnológico enorme (Iov, 2006; Heier, 2014).

Este desafío ha llevado a una nueva tendencia en la generación de energía, la generación local utilizando fuentes de energías alternativas como micro turbinas, celdas fotovoltaicas, aerogeneradores, celdas de combustibles, etc. Este tipo de generación (local + energías alternativas) es llamado generación distribuida renovable. En forma general, el término "generación distribuida" ha sido concebido para distinguir este concepto en contrapartida a la generación convencional centralizada.

Dentro de las energías renovables, la eólica y la solar son las de mayor crecimiento. En las últimas tres décadas, en lo referente al recurso eólico en particular, se ha desarrollado un marco teórico sólido para comprender la naturaleza del viento y la manera de obtener energía aprovechable a partir del mismo (Ackermann, 2012; Heier, 2014). A partir de las previsiones del aumento del consumo de energía eléctrica para el año 2020 se han propuesto diferentes escenarios para analizar la potencia instalada de energía eólica instalada en la Unión Europea. Uno de los escenarios centrales prevé que la capacidad instalada aumentará un $64 \%$ comparada con la producción del año 2013 y abastecerá aproximadamente el $15 \%$ 
de la demanda total de energía (EWEA, 2014).

La naturaleza variable del viento y la manera en que las turbinas eólicas extraen su energía, comprometen la calidad de la potencia eléctrica obtenida, en relación a los estándares vigentes. A los efectos de mantener la generación dentro de dichos estándares, se hace indispensable la implementación de acciones tendientes a evitar y/o corregir tales apartamientos. La variabilidad del recurso eólico renovable no sólo perturba la calidad de la potencia eléctrica generada, sino que también puede comprometer el desempeño del sistema de generación en su conjunto. En este sentido, es indispensable el análisis de tales efectos para asegurar el comportamiento integral del sistema. Estos inconvenientes se ven incrementados cuando los sistemas de generación eólica se conectan junto con otros sistemas de generación convencional y no convencional en redes distribuidas. A ello se deberá agregar también el estudio de situaciones particulares como los huecos de tensión y cortocircuitos que afectan fuertemente la estabilidad de cualquier sistema eléctrico (Anderson, 2003; Kundur, 1993). Las leyes de control desarrolladas en redes eléctricas han mostrado su eficacia en contribuir a estabilizarlas desde el comienzo mismo de la generación. En este sentido, algunos sistemas de generación distribuida fueron estudiados empleando técnicas lineales (Tang et al., 2009, 2014). Sin embargo, en un contexto de dinámicas cambiantes y complejas como los sistemas de generación distribuida usualmente requieren técnicas más avanzadas de control. En este sentido, es importante explorar el empleo de herramientas no lineales de control a los efectos de maximizar el empleo del recurso eólico a la vez que se asegure la estabilidad del sistema. En este contexto, las herramientas provenientes de la Pasividad garantizan la estabilidad (por Lyapunov) de las redes eléctricas.

En estos últimos 15 años, se ha realizado un gran esfuerzo por parte de diferentes investigadores con respecto al desarrollo de técnicas no lineales de control que explotan la estructura del sistema. Las ideas del control basado en pasividad emplean conceptos básicos de energía. Efectivamente un sistema físico puede ser estudiado como un subsistema que intercambia energía con el medio y es común que el sistema dinámico pueda ser visto como un dispositivo que transforma la energía. En particular, las técnicas basadas en pasividad, definen una metodología para la síntesis de un controlador mediante una función de energía y en algunos casos inyectando amortiguamiento (Ortega y García-Canseco, 2004). Además del enfoque basado en la energía del sistema otro marco de trabajo fue desarrollado recientemente con el objetivo de balancear la potencia del sistema. Este nuevo marco de trabajo, llamado moldeo de potencia Jeltsema y Scherpen (2007); García-Canseco et al. (2007), emplea para la modelización del sistema las expresiones de Brayton-Moser Brayton y Moser (1964a,b) y las propiedades de la función de potencial mixto para producir una función de Lyapunov. Además, las variables que se utilizan para realizar la modelización suelen ser corrientes y tensiones. Este último es el enfoque empleado en esta tesis para abordar problemas de sistemas de conversión de energía eólica y del control de un sistema de almacenamiento de energía en el marco de una micro red.

\subsection{Organización y estructura de la tesis}

Este trabajo de tesis ha sido organizado de la siguiente manera. Los Capítulos 2, 3 y 4 introducen los conceptos de los sistemas de conversión de energía eólica, las redes eléctricas y el control vectorial. En el Capítulo 5 se desarrolla la descripción y el desarrollo de las técnicas de control por pasividad y por moldeo de potencia mientras que en el Capítulo 6 se exponen 
las aplicaciones desarrolladas. Finalmente, en el Capítulo 7 se presentan las conclusiones y las futuras líneas de investigación.

En el Capítulo 2 se presentan los fundamentos básicos referidos a la energía eólica y a su conversión. Se presenta un modelo de una turbina eólica y se comentan diferentes topologías de los sistemas de conversión de energía eólica. Además, se indican los requerimientos de red para la integración de sistemas de generación de energía eólica.

En el Capítulo 3 se desarrollan los conceptos básicos para el análisis de una red eléctrica. Se presentan las expresiones de intercambio de potencias entre dos nodos y se describen los modelos estáticos y dinámicos para una carga conectada a una red eléctrica. Estas cargas dinámicas pueden representar el comportamiento de los motores de inducción conectados a la red eléctrica. Se presenta también un modelo del generador sincrónico con sus controladores respectivos.

En el Capítulo 4 se desarrollan las ideas del control vectorial aplicado a una máquina de inducción. Estos conceptos han sido empleados para el análisis de una máquina eléctrica. Con el objetivo de controlar la máquina de inducción de rotor bobinado se presentan las técnicas de control vectorial empleando los marcos de referencia de flujo estatórico y según el flujo de red.

En el Capítulo 5 se introducen las ideas básicas referidas a pasividad y las propiedades de los sistemas pasivos. A partir de los conceptos básicos de conservación y de balance de la energía se presenta la modelización de un sistema físico empleando estas ideas. En este sentido, la teoría de sistemas hamiltonianos presenta un marco de trabajo sistematizado para el análisis y el control de sistemas. En particular, se presentan las técnicas de moldeo de la energía del sistema utilizando inyección de amortiguamiento y de moldeo de potencia las cuales brindan mayor flexibilidad en la obtención de la función de Lyapunov. Finalmente, a partir de la equivalencia entre los formalismos basados en la teoría de puertos hamiltonianos y las expresiones de Brayton-Moser se presenta la inclusión de un control integral para un controlador por moldeo de potencia.

En el Capítulo 6, en el contexto de los sistemas de generación distribuida renovable se considera el control de un generador asincrónico de rotor bobinado para un aerogenerador con conexión a red. Empleando los conceptos del control vectorial para una máquina de rotor bobinado y empleando las ideas de pasividad/moldeo de potencia se presenta un controlador para la potencia activa y reactiva. La ley de control propuesta se la evalúa en diferentes escenarios de funcionamiento del aerogenerador. Además, se presenta una micro red que opera en forma aislada. Con el objetivo de aumentar la calidad del suministro eléctrico se considera un sistema de almacenamiento en la estructura de la misma. En esta estructura en particular se considera el control del sistema de almacenamiento de energía mediante moldeo de potencia con el objetivo de controlar la potencia activa y reactiva que entrega al punto de conexión.

Finalmente, en el Capítulo 7 se presentan las conclusiones de este trabajo y las futuras líneas de investigación. 


\subsection{Principales aportes}

Los aportes originales presentados en esta tesis son los siguientes:

- Aplicar nuevas herramientas de control no lineal en contraposición a las capacidades limitadas de las herramientas lineales en el contexto de los sistemas de generación distribuida renovable.

- Diseño de un controlador para el control de la potencia activa para un aerogenerador equipado con un generador asincrónico de rotor bobinado mediante los conceptos de control vectorial y la técnica basada en pasividad por moldeo de potencia.

- A partir de los resultados anteriores empleando las ideas de un aerogenerador equivalente y el control por balance de potencia se desarrolla un controlador para las potencias activa y reactiva que intercambia un parque eólico vinculado a una red.

- Desarrollo de una acción integral para los controladores de las potencias activa y reactiva para los generadores de rotor bobinado de los aerogeneradores que conforman el parque eólico considerado sin perder la estructura de las ecuaciones de Brayton-Moser.

- Diseño de dos estrategias de control basadas en Moldeo de Potencia para el generador de rotor bobinado considerando que el generador opere neutral a la red y otra que regula la tensión en caso de que exista una variación en el punto de conexión.

- Diseño de un controlador para un sistema de almacenamiento de energía que interviene en una micro red, que opera en forma aislada, mediante moldeo de potencia garantizando la estabilidad del sistema no lineal mediante una función de Lyapunov.

- Diseño de una estrategia de acondicionamiento de la referencia para la potencia que entrega el sistema de almacenamiento de energía.

- En los análisis y evaluaciones de los controladores de Moldeo de Potencia, se utiliza una carga dinámica para representar la gran capacidad instalada de motores de inducción en una red eléctrica típica.

- A través de la síntesis por moldeo de potencia de los controladores empleados en un sistema de conversión de energía eólica y en una micro red se muestra que puede incrementarse la zona de funcionamiento estable de cada sistema.

Finalmente, cabe destacar que los resultados de esta tesis han dado origen a una serie de artículos publicados en revistas internacionales y congresos que a continuación se detallan

- Peña, R.R, Fernández, R.D., Mantz, R.J., Battaiotto, P.E. "Power-based control with integral action for wind turbines connected to the grid". International Journal of Control. Volume 88, número 10, pp. 2143-2153, 2015. DOI:10.1080/00207179.2015.1039064.

- Peña, R.R, Fernández, R.D., Mantz, R.J. "Passivity control via Power Shaping of a wind turbine in a dispersed network". International Journal of Hydrogen Energy. Volumen 39, número 16, pp. 8846-8851, 2014, DOI: http://dx.doi.org/10.1016/j.ijhydene.2013.12.023. 
- Peña, R.R., Fernández, R.D. Mantz, R.J., Battaiotto, P.E. "Passivity-based control of energy storage units in Distributed Generation Systems". 2015 IEEE PES Innovative Smart Grid Technologies Latin America (ISGT LATAM),(2015 ISGT-LA), pp. 1-6, 2015 .

- Peña, R.R, Fernández, R.D., Mantz, R.J. "Control por moldeo de potencia de un aerogenerador en generación distribuida", Reunión de Trabajo en Procesamiento de la Información y Control (RPIC 2013). 2013.

- Peña, R.R, Fernández, R.D., Mantz, R.J."Control por pasividad de un aerogenerador en una red dispersa", 5to. Congreso Nacional - 4to. Congreso Iberoamericano Hidrógeno y fuentes sustentables de energía Córdoba, Argentina, 10 - 14 de junio de 2013.

Adicionalmente los siguientes trabajos, que también forman parte del cuerpo de la tesis, han sido desarrollados como colaborador.

- Fernández, R.D., Valenciaga F., Peña R.R. "Nonlinear Control of a Permanent Magnet Synchronous Wind Generator", 2015 IEEE PES Innovative Smart Grid Technologies Latin America (ISGT LATAM),(2015 ISGT-LA), pp. 236-239, 2015.

- Toccaceli, G.M., Peña, R.R., Cendoya, M. Battaiotto, P.E. "RDG System in Remote Areas with Utility or Local Feed Topology and Operation Strategy". Journal of Mechanics Engineering and Automation. Volumen 4, número 7, pp. 575-583, 2014.

- Toccaceli, G.M. and Peña, R.R. and Cendoya, M.G. and Battaiotto, P.E. "Sistema de generación distribuida renovable con capacidad de operación en isla", AADECA 2012. 2012 .

- Toccaceli, G.M., Battaiotto, P.E., Cendoya, M.G., Peña, R.R. "GDR in weak grid for desalination plant. System topology and control strategy", Transmission and Distribution: Latin America Conference and Exposition (T D-LA), 2012 Sixth IEEE/PES, pp. 1-8, 2012. 


\section{Capítulo 2}

\section{RECURSO EÓLICO}

\section{Resumen}

En este capítulo se exponen los conceptos básicos referidos a la energía eólica y a su conversión. En este sentido, se introducen los aspectos generales referidos al recurso eólico y como las turbinas eólicas extraen energía del viento. Se introducen los requerimientos para la integración de granjas eólicas a un sistema interconectado.

\subsection{Introducción}

La energía es el principal motor de crecimiento económico de un país y es vital para lograr el desarrollo económico y social.

El aumento de la penetración de generación empleando energías renovables ha ido creciendo en estas últimas décadas. Actualmente, es posible montar aerogeneradores de potencias elevadas lo que permite construir granjas eólicas con una capacidad de potencia instalada importante. Estas granjas eólicas deben cumplir con códigos de red para su conexión a la red eléctrica (Tsili y Papathanassiou, 2009). Al utilizar fuentes de energía renovables se disminuye la contaminación en la producción de energía eléctrica. En Shanker y Singh (2012) se estima que la producción de energía utilizando un recurso renovable como el viento podría producir el $12 \%$ de la electricidad necesaria a nivel mundial para el 2020, evitando la emisión de 1,5 billones de toneladas de $\mathrm{CO}_{2}$ por año. En este sentido, la utilización de energía eólica para la producción de energía eléctrica resulta una alternativa viable para disminuir la utilización de combustibles fósiles. 


\subsection{Naturaleza del viento}

El viento puede considerarse como el movimiento de una masa de aire en la atmósfera originado principalmente por diferencias de temperatura. Estas diferencias que dan lugar a un gradiente de presión, son por causa del calentamiento desigual de la superficie terrestre por el sol. En las zonas próximas al ecuador las masas de aire son calentadas más que en el resto del planeta. Así, la masa de aire asciende y posteriormente se dirige a zonas de menor temperatura.

Los efectos del calentamiento diferencial disminuyen para latitudes superiores a $30^{\circ} \mathrm{N} \mathrm{y}$ $30^{\circ} \mathrm{S}$ donde predominan los vientos del oeste. Esta circulación de aire es debida a las fuerzas de coriolis, es decir como resultado de la rotación de la tierra, de forma tal que la capa superior del viento se desvía hacia el este mientras que la capa inferior se desvía hacia el oeste. Estos flujos de aire de gran escala constituyen el viento geostrófico. En las regiones por debajo de los 100 metros existen los vientos llamados de superficie donde el movimiento de la masa de aire es perturbado por las características del terreno (rugosidad, orografía, obstáculos, etc ) (Burton et al., 2011).

Así, el viento cerca de la superficie de la tierra resulta de la combinación del viento geostrófico y el viento local.

La previsibilidad del viento es importante para la integración de grandes cantidades de energía eólica a la red eléctrica. Basado en registros a corto y a largo plazo, es posible construir un gráfico espectral del viento llamado espectro de Van der Hoven (Van der Hoven, 1957). Un espectro típico es presentado en la Figura 2.1, en el cual $\Phi\left[\frac{m^{2}}{s^{2}}\right]$ es la densidad espectral de potencia del viento.

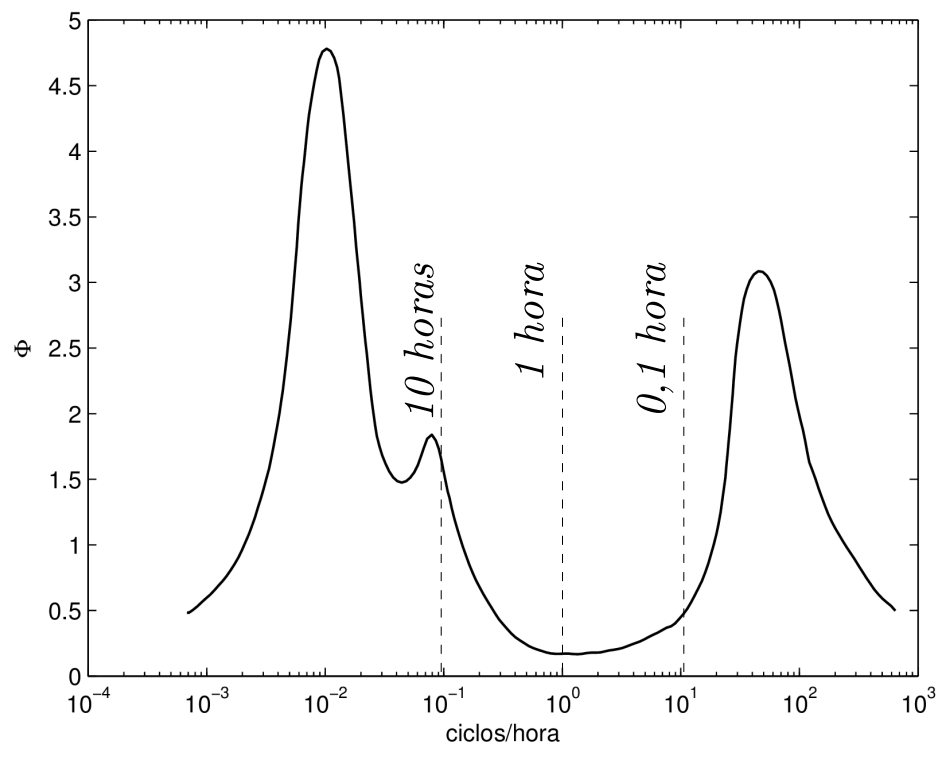

Figura 2.1: Espectro de Van der Hoven.

En la zona de baja frecuencia de la Figura 2.1 se puede observar un pico de energía debido a efectos diurnos. Mientras que en escalas de tiempo más pequeños se producen variaciones en el orden del minuto o los segundos conocidas como turbulencias, las cuales pueden tener un 
impacto significativo en el diseño de las turbinas eólicas debido a las cargas estructurales como así también en la calidad de la energía entregada a la red eléctrica. Como las características del régimen del viento son variables en cada lugar geográfico, el espectro de Van der Hoven no puede ser aplicado universalmente, sin embargo la forma de la distribución se repite. Luego el concepto de hueco espectral en el rango de 10 minutos a dos horas es útil al realizar suposiciones del régimen del viento (Olimpo et al., 2009).

\subsubsection{Distribución estadística}

Mediciones recolectadas durante años ayudan a caracterizar el recurso, y en particular predecir la velocidad media del viento. Las variaciones de la velocidad pueden ser caracterizadas anualmente en términos de una distribución de probabilidad llamada de Weibull.

La distribución de Weibull es dada por:

$$
P(v)=\left(\frac{k}{c}\right)\left(\frac{v}{c}\right)^{k-1} e^{-\left(\frac{v}{c}\right)^{k}},
$$

donde $k$ y $c$ son coeficientes de forma y escala respectivamente y pueden ser ajustados para que coincidan con datos experimentales del viento $(v)$ (Bianchi et al., 2006). Los coeficientes de la distribución estadística varían según el lugar geográfico dependiendo de las condiciones climáticas, superficie, etc. En los trabajos Arraña et al. (2014) y Palese et al. (2009) se presentan diferentes valores de los coeficientes de forma y de escala de la distribución de Weibull para diferentes localizaciones en la República Argentina.

En la Figura 2.2 se muestra la distribución de Weibull para diferentes valores de $k$. El coeficiente $c$ es 7,3 para las curvas de la Figura 2.2. Un caso particular de la expresión (2.1) es cuando $k=2$, el cual da lugar a la distribución de Rayleigh.

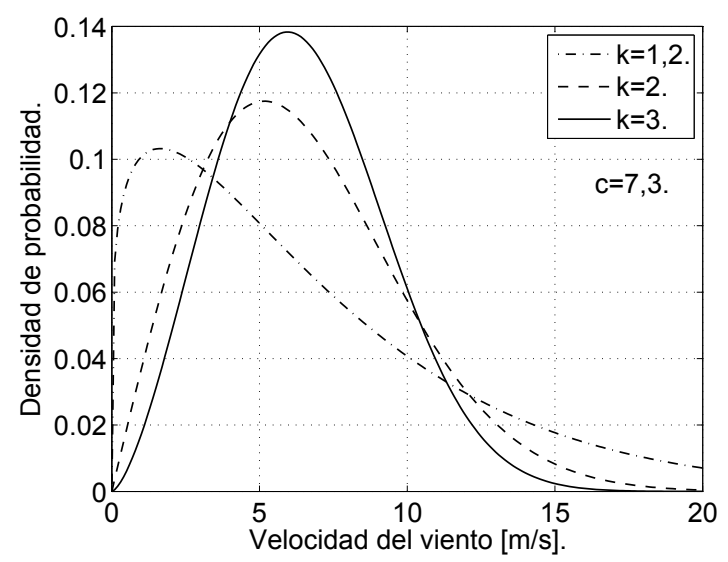

Figura 2.2: Distribución de Weibull $k=1,2, k=2, k=3$ en líneas de trazo y punto, trazo y llena, respectivamente.

Como se observa en la Figura 2.2 para $k<3$ la distribución está sesgada hacia las velocidades bajas. 


\subsubsection{Energía y potencia disponible en el viento}

La energía cinética almacenada en un flujo de aire por unidad de volumen es:

$$
E_{k}=\frac{1}{2} \rho v^{2},
$$

donde $\rho$ es la densidad del aire $\left[\frac{\mathrm{kg}}{\mathrm{m}^{3}}\right]$ y $v$ es la velocidad del viento $\left[\frac{\mathrm{m}}{\mathrm{s}}\right]$.

Luego, la potencia disponible del viento está definida como:

$$
P_{v}(v)=\frac{1}{2} \rho A v^{3}
$$

donde $A$ es el área considerada. De la expresión (2.3), se puede observar que la potencia disponible en el viento varía con el cubo de su velocidad.

\subsubsection{Turbulencias}

Las turbulencias se corresponden con el pico de alta frecuencia, el cual se puede observar en la gráfica de Van der Hoven (Figura 2.1) y se encuentran en el rango de 5 segundos a 10 minutos. Principalmente son producidas por dos causas:

- Fricción con la superficie de la tierra, debidas a que el flujo de aire experimenta variaciones al atravesar diferentes características topográficas del terreno.

- Efectos térmicos, es decir, variaciones de temperaturas que producen el movimiento de una masa de aire verticalmente.

A menudo el efecto de la fricción y el efecto térmico están relacionados.

\subsection{Turbina eólica}

La turbina eólica es un dispositivo que puede intercambiar cantidad de movimiento con el viento. La energía capturada por la turbina puede ser utilizada para moler granos, impulsar una bomba de agua o impulsar un generador eléctrico. Particularmente, es de interés para este trabajo el empleo de aerogeneradores para producir energía eléctrica.

Los componentes principales de un aerogenerador son la turbina eólica, la caja multiplicadora, el generador, el transformador y opcionalmente convertidores electrónicos de potencia. Un esquema básico es presentado en la Figura 2.3 (Chen y Blaabjerg, 2009).

Según el tipo de generador eléctrico utilizado en el diseño de un aerogenerador se debe seleccionar un convertidor electrónico de potencia asociado al mismo, aunque, en los generadores de velocidad fija la inclusión de un convertidor electrónico es opcional. A menudo los aerogeneradores son instalados con otros aerogeneradores formando un parque o una granja eólica. Los grandes parques eólicos pueden presentar una contribución significativa de energía a la redes eléctricas y hoy en día pueden jugar un papel importante en la calidad y la disponibilidad de la energía eléctrica. Por lo tanto, para satisfacer los requerimientos del sistema donde serán conectadas las unidades de generación tienen que cumplir con los códigos de red (Chen y Blaabjerg, 2009). 


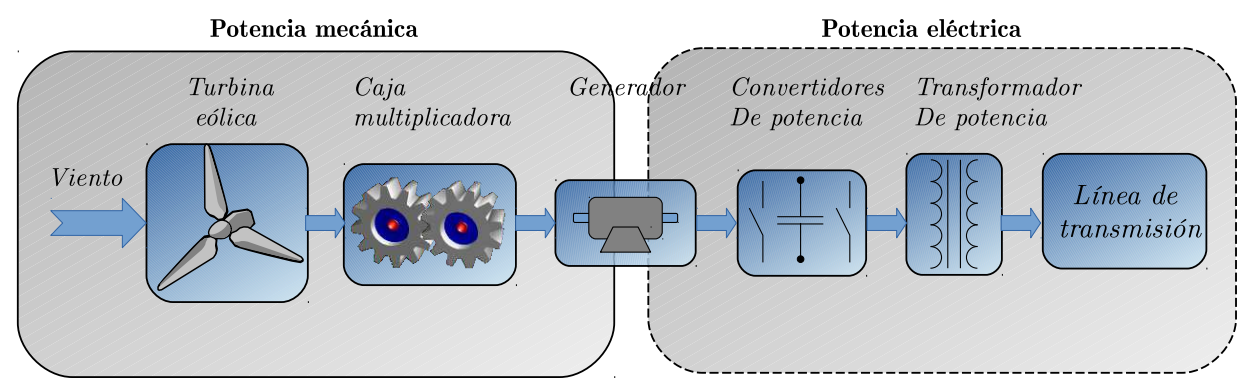

Figura 2.3: Elementos principales de un aerogenerador.

\section{Tipos de turbinas eólicas}

Para capturar la energía proveniente del viento (energía cinética) y transformarla mediante un generador en energía eléctrica, diferentes diseños han sido desarrollados en (Heier, 2014).

En adición a la eficiencia de conversión, los aerogeneradores deben ser diseñados para soportar los esfuerzos y cargas aerodinámicas. En este sentido cabe señalar que el número de palas, la velocidad del rotor, la relación de velocidad de punta de pala, la geometría de las palas y el control de paso de palas también conocido como control de "pitch" juegan un rol importante (Heier, 2014).

Existen numerosas formas de clasificar turbinas eólicas. Una primera clasificación es si el eje del rotor está en posición vertical u horizontal. La turbina más utilizada de eje vertical es el modelo Darrieus (Bianchi et al., 2006). Sin embargo, el mercado de aerogeneradores de potencias elevadas ha ido convergiendo a turbinas eólicas de eje horizontal de 3 palas. Esta elección es esencialmente motivada por razones estructurales y de eficiencia. En la Figura 2.4 se puede observar un esquema de un aerogenerador de eje horizontal con sus partes constitutivas. La góndola contiene en su interior la caja multiplicadora, los frenos de emergencia, el generador, los sistemas de control, etc. Además, la góndola está equipada con un anemómetro que mide la velocidad del viento y también la dirección.

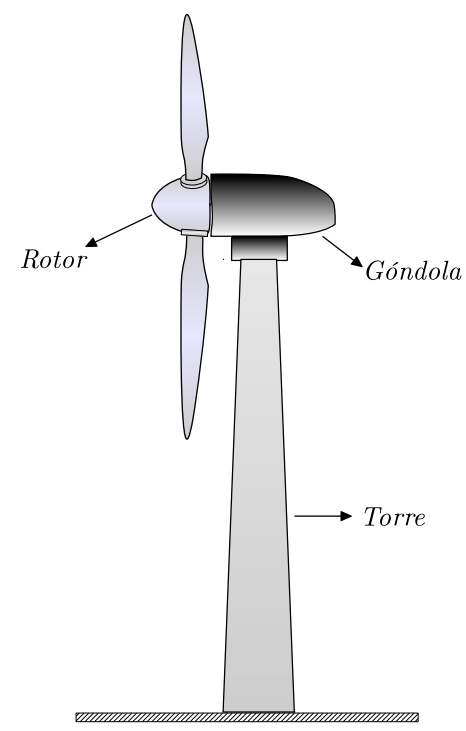

Figura 2.4: Turbina eólica de eje horizontal. 
Los aerogeneradores de tamaño considerable generalmente se controlan para operar a velocidad variable mientras que los de pequeña escala suelen operar a velocidad fija. Las turbinas eólicas pueden estar orientadas de cara al viento (barlovento) o contra el viento (sotavento). Actualmente la mayoría de las turbinas están orientadas a barlovento.

\section{Variación de la velocidad con respecto a la altura}

Los perfiles de velocidad de viento varían con la altura. Es decir la pala que se encuentra más cerca del nivel del suelo experimenta una velocidad de viento menor que las otras que están a una altura mayor. Esta situación se grafica en la Figura 2.5. Además, ráfagas y cambios en la velocidad del viento no afectan al rotor en el mismo instante. Para velocidades de viento que se encuentran en el rango operacional de la turbina y exceden los $4 \mathrm{~m} / \mathrm{s}$ es posible utilizar la siguiente expresión para la velocidad en función de la altura:

$$
v(h)=v_{10}\left(\frac{h}{h_{10}}\right)^{a},
$$

donde $0,14 \leq a \leq 0,17$ es llamado exponente de Hellman, $v_{10}$ es la velocidad determinada para una altura $h_{10}=10 \mathrm{~m}$ y $h$ corresponde a la posición de cada elemento de las palas (Heier, 2014). En la Figura 2.5 se gráfica la expresión (2.4) para $h_{10}=10 \mathrm{~m}, v_{10}=9,2 \mathrm{~m} / \mathrm{s}$ y $a=0,16$. También se puede observar en la Figura 2.5 la diferencia de velocidades $(\Delta v)$ que experimenta la pala entre los extremos inferior y superior.

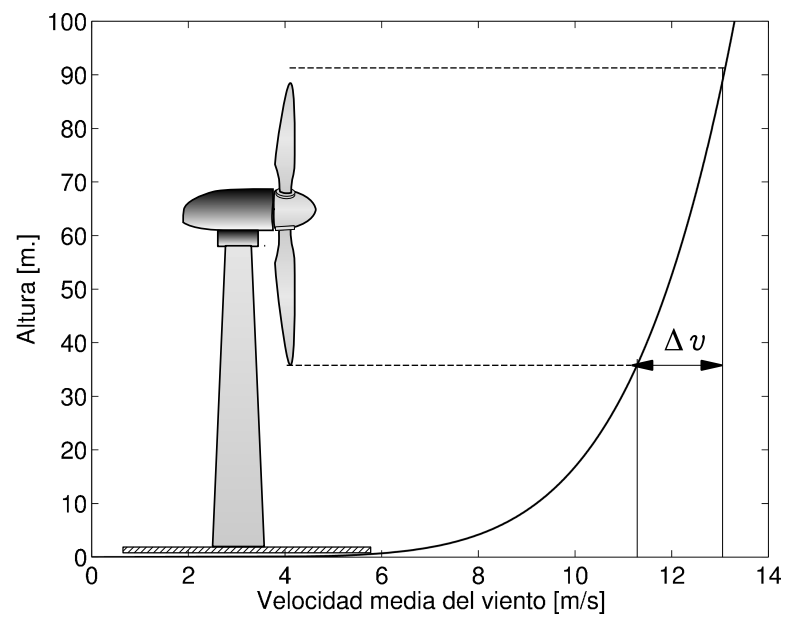

Figura 2.5: Perfil vertical de la velocidad del viento según la altura, según aproximación (2.4).

\section{Eficiencia de conversión de una turbina eólica}

A continuación, a partir de la teoría del disco actuador, se describe la eficiencia de conversión de una turbina eólica y mediante la teoría del elemento de pala es posible estudiar las fuerzas aerodinámicas con el objetivo de obtener una expresión para la potencia extraída del viento por el aerogenerador. En efecto, el método del disco actuador nos permite obtener de una forma simple la energía extraída y además un límite teórico para la extracción de energía por parte de una turbina eólica. Para estudiar el comportamiento aerodinámico de una turbina eólica se considera que el rotor se comporta como un disco que extrae energía 
del viento, Figura 2.6. Además, se asume un tubo de corriente que encierra al rotor eólico y que el viento que incide en el disco es uniforme. El viento en la Figura 2.6 ingresa por la superficie $A_{\infty}$ con una velocidad $v_{\infty}$. El aire al atravesar el disco actuador pierde velocidad ya que cede parte de su energía al disco actuador. Luego de atravesar el disco el área de la sección considerada es mayor $A_{w}$ y su velocidad es indicada como $v_{w}$.

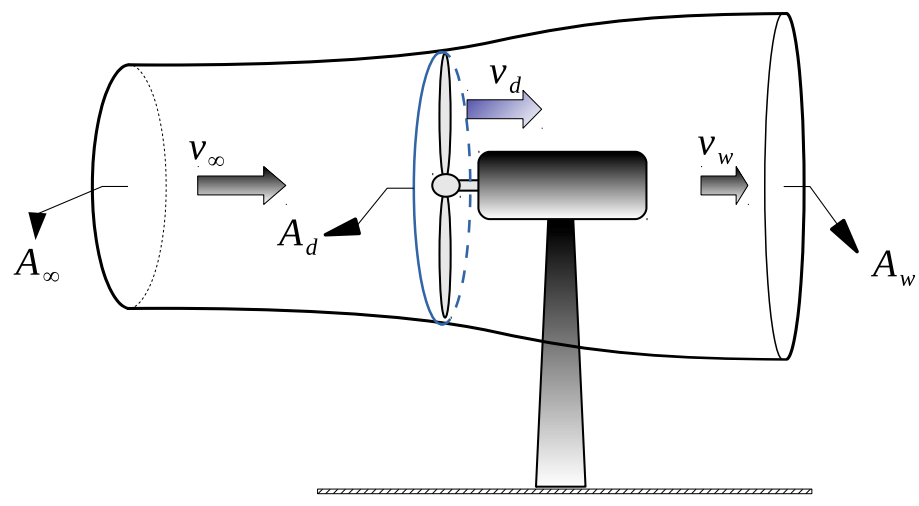

Figura 2.6: Tubo de corriente de aire.

Siguiendo a Burton et al. (2011) se puede caracterizar la habilidad de una turbina eólica para capturar la energía del viento a través del coeficiente de potencia, que se define como la potencia que puede extraer la turbina eólica sobre la potencia total disponible en el viento, dada por la expresión (2.3), es decir:

$$
C_{p} \approx \frac{P_{d}}{P_{v}}
$$

Con $P_{d}$ dada por:

$$
P_{d}=2 \rho A_{d} v_{\infty}^{3} a(1-a)^{2},
$$

y $a$ es un factor que tiene en cuenta como se reduce la velocidad en el disco actuador, concretamente, $v_{d}=v_{\infty}(1-a)$. Entonces, el coeficiente de potencia $\left(C_{p}\right)$ resulta:

$$
C_{p}=\frac{P_{d}}{P_{v}}=\frac{2 \rho A_{d} v_{\infty}^{3} a(1-a)^{2}}{\frac{1}{2} \rho v_{\infty}^{3} A_{d}} \Rightarrow 4 a(1-a)^{2} \Rightarrow 4 a-8 a^{2}+4 a^{3} .
$$

Con el objetivo de calcular el máximo de la expresión del coeficiente de potencia se puede derivar (2.7) con respecto a la variable $a$ e igualarla a cero:

$$
\frac{d C_{p}}{d a}=4-16 a+12 a^{2}=0 .
$$

Las soluciones para la ecuación (2.8) son $a_{1}=\frac{1}{3}$ y $a_{2}=1$. El máximo de la expresión (2.7) se obtiene para $a=\frac{1}{3}$. Entonces, reemplazando en la ecuación (2.7) con el valor previamente calculado de la variable $a$, el valor máximo para el coeficiente de potencia es:

$$
C_{P \max }=\frac{16}{27}=0,593 .
$$

Este valor, conocido como el límite de Betz establece que una turbina eólica no podrá extraer más que el $59.3 \%$ de la energía total del viento.

El coeficiente de potencia para turbinas eólicas de potencia está en el rango del $25 \%$ al $45 \%$ (Olimpo et al., 2009). Los datos del coeficiente de potencia son provistos por el fabricante de la turbina eólica (Bianchi et al., 2006). 
Para lograr obtener la máxima potencia eléctrica en un aerogenerador se emplean turbinas con palas de secciones transversales similares a la utilizadas en perfiles alares. La Figura 2.7 muestra un corte típico de una pala utilizada en un aerogenerador. Las velocidades que se muestran en la Figura 2.7 son la velocidad real del viento que incide en la pala $V(1-a)$, la componente de la velocidad del viento en el plano del rotor $v_{p}$ y por último la velocidad relativa $V_{\text {rel }}$ que resulta de la suma de las dos anteriores.

Los perfiles alares utilizados están sometidos a diferentes cargas, las cuales deben ser consideradas en el momento del diseño de una turbina. En la Figura 2.7 se pueden observar las dos fuerzas (fuerza de arrastre y fuerza de sustentación) que intervienen en la generación del par responsable de que la turbina eólica gire.

La fuerza de arrastre $\left(f_{D}\right)$, está en la dirección de $V_{\text {rel }}$ y la fuerza de sustentación $\left(f_{L}\right)$ la cual es ortogonal a $f_{D}$. Estas fuerzas son expresadas como:

$$
\begin{aligned}
f_{D} & =\frac{\rho c}{2} V_{r e l}^{2} C_{D}(\alpha), \\
f_{L} & =\frac{\rho c}{2} V_{r e l}^{2} C_{L}(\alpha),
\end{aligned}
$$

donde $c$ es la cuerda del elemento de la pala, $C_{L}$ y $C_{D}$ son los coeficientes de sustentación y de arrastre respectivamente que son función del ángulo de incidencia $\alpha$ definido como:

$$
\alpha=\phi-\beta,
$$

donde $\phi$ es el ángulo entre la dirección de la velocidad $\left(V_{\text {rel }}\right)$ y el plano del rotor y $\beta$ es el ángulo de pitch, el cual es medido entre el plano del rotor y la cuerda (Figura 2.7).
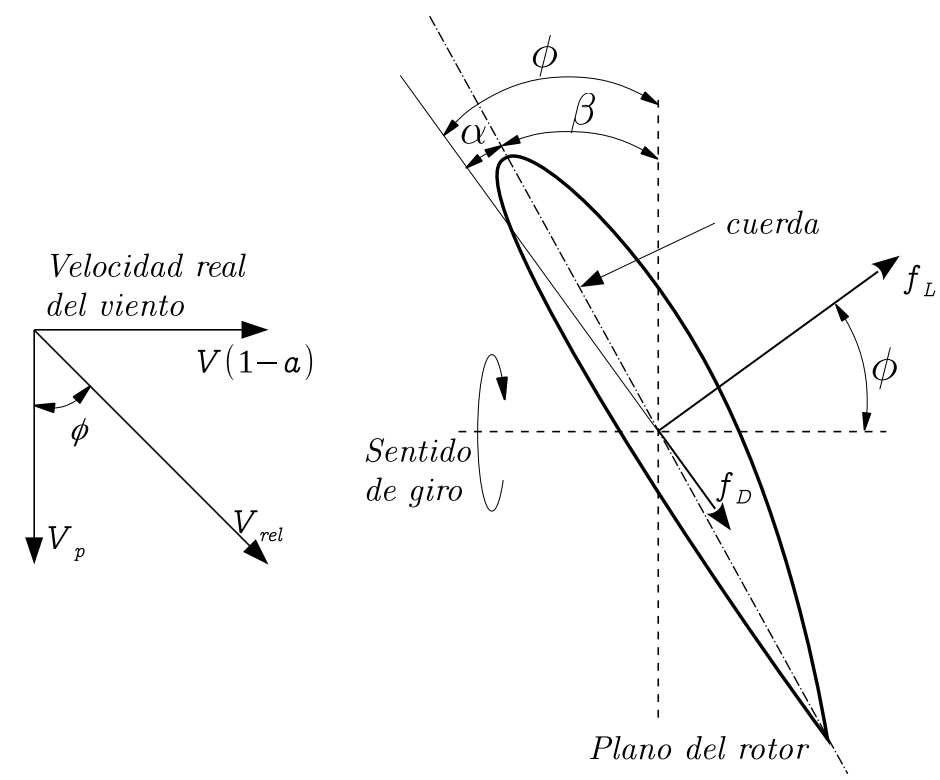

Figura 2.7: Velocidades y fuerzas en un elemento de pala de una turbina eólica.

El par desarrollado en el eje de la turbina eólica que resulta de la integración de las componentes de las fuerzas $f_{L}$ y $f_{D}$ en el plano del rotor a lo largo de las palas, es dado por:

$$
T_{t}=\frac{1}{2} \rho \pi R^{3} C_{Q}(\lambda, \beta) v^{2},
$$


donde

$$
C_{Q}(\lambda, \beta)=\frac{C_{p}(\lambda, \beta)}{\lambda}
$$

es el coeficiente de par, que de la misma forma que el coeficiente de potencia $C_{p}(\lambda, \beta)$ depende de la relación de punta de pala $\lambda$ y del ángulo $\beta$.

La relación de punta de pala se define como:

$$
\lambda=\frac{\Omega R}{v},
$$

donde $\Omega$ es la velocidad del rotor, $R$ es el radio de la pala y $v$ es la velocidad del viento. De esta manera, la relación de punta de pala relaciona la velocidad tangencial en el extremo de las palas con la velocidad del viento. La relación de velocidad de punta de pala puede ir de 50 a $150 \mathrm{~m} / \mathrm{s}$. Aunque, debido a problemas de ruido $\lambda$ no suele superar $\operatorname{los} 75 \mathrm{~m} / \mathrm{s}$. Esta relación es crítica para establecer las dimensiones de las palas tanto en aerogeneradores pequeños como en grandes (Heier, 2014).

De esta forma, la potencia que puede extraer una turbina eólica está dada por la expresión:

$$
P_{t}=C_{p}(\lambda, \beta) P_{v}=C_{p}(\lambda, \beta) \frac{1}{2} \rho A v^{3} .
$$

En las Figuras 2.8 y 2.9 se puede observar la potencia mecánica y el par de una turbina eólica en función de la velocidad angular del eje del rotor para diferentes velocidades de viento, respectivamente.

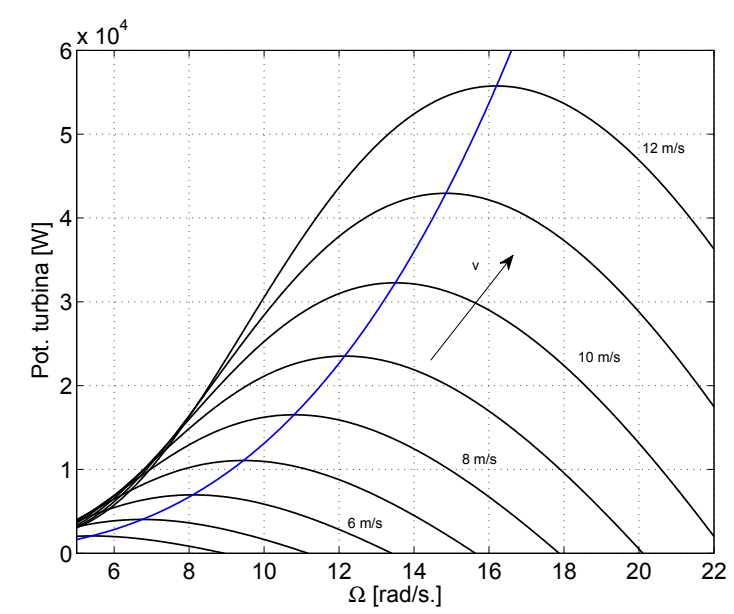

Figura 2.8: Potencia mecánica de una turbina eólica y curva de la potencia óptima (línea azul) desarrollada.

De la expresión (2.15) con el valor de relación de punta de pala óptimo $\left(\lambda_{0}\right)$, para el cual $C_{p}(\lambda, \beta)$ es máximo, podemos despejar la variable " $v$ " de la siguiente forma:

$$
v=\frac{\Omega R}{\lambda_{0}},
$$

y reemplazando en la ecuación (2.16) podemos obtener la expresión para la potencia óptima:

$$
P_{0}=k_{0} \Omega^{3},
$$


y el correspondiente par:

$$
T_{0}=k_{0} \Omega^{2}
$$

con

$$
k_{0}=\frac{1}{2} \rho A C_{p \max }\left(\frac{R}{\lambda_{0}}\right)^{3}
$$

donde $C_{p \max }=C_{p}\left(\lambda_{0}, \beta_{0}\right)$ es el valor máximo del coeficiente de potencia. En las Figuras 2.8 y 2.9 se puede observar con trazo de color azul las expresiones referidas a la potencia (2.18) y el par (2.19) óptimos desarrollados por una turbina eólica para diferentes velocidades de viento. También se distingue que la velocidad $\Omega$ del máximo de potencia no corresponde con la velocidad del máximo de par.

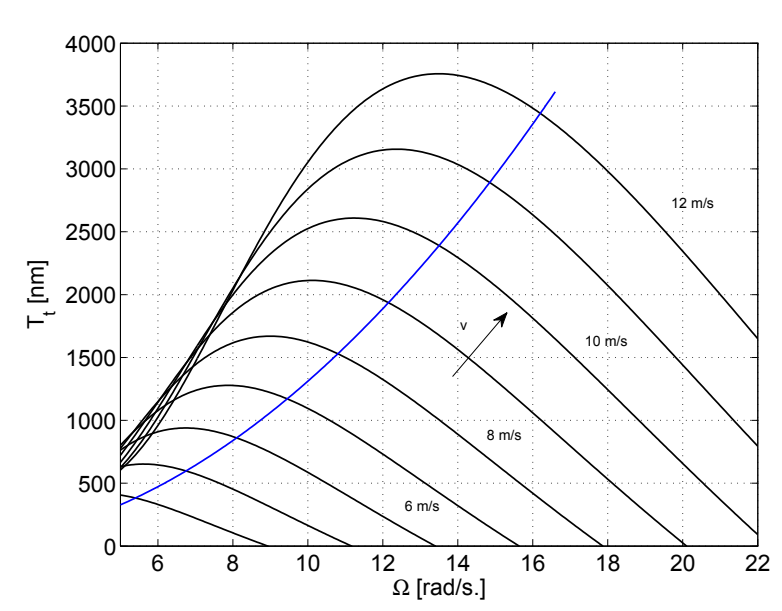

Figura 2.9: Par mecánico desarrollado por una turbina eólica para diferentes velocidades de viento y curva del par óptimo (línea azul).

\section{Aproximación del coeficiente de potencia por una función analítica.}

La expresión correspondiente al coeficiente de potencia $\left(C_{p}(\lambda, \beta)\right)$ de una turbina eólica real puede ser aproximada por una superficie no lineal. En Heier (2014) se presenta un modelo del coeficiente de potencia $\left(C_{p}(\lambda, \beta)\right)$ mediante la aproximación de una función analítica dada por:

$$
C_{P}(\lambda, \beta)=c_{1}\left(c_{2}-c_{3} \beta-c_{4} \beta^{x}-c_{5}\right) e^{-c_{6}(\lambda, \beta)},
$$

donde $c_{1}, c_{2}, c_{3}, c_{4}, c_{5}$ y $c_{6}$ son coeficientes que deben ajustarse para cada turbina.

En la Figura 2.10 se pueden observar diferentes curvas del coeficiente de potencia $\left(C_{p}\right)$ en función de $\lambda$ para diferentes valores del ángulo de pitch $(\beta)$ de las palas. La gráfica corresponde a un aerogenerador de una potencia nominal de 2,5 MW del tipo MOD-2, cuyas características y parámetros son presentados en Heier (2014), donde $c_{1}=0,5, c_{2}=\frac{116}{\lambda_{i}}, c_{3}=0,4, c_{4}=0$, $x=1,5, c_{5}=5, c_{6}=\frac{21}{\lambda_{i}} \mathrm{y}$

$$
\frac{1}{\lambda_{i}}=\frac{1}{\lambda+0,08 \beta}-\frac{0,035}{\beta^{3}+1}
$$




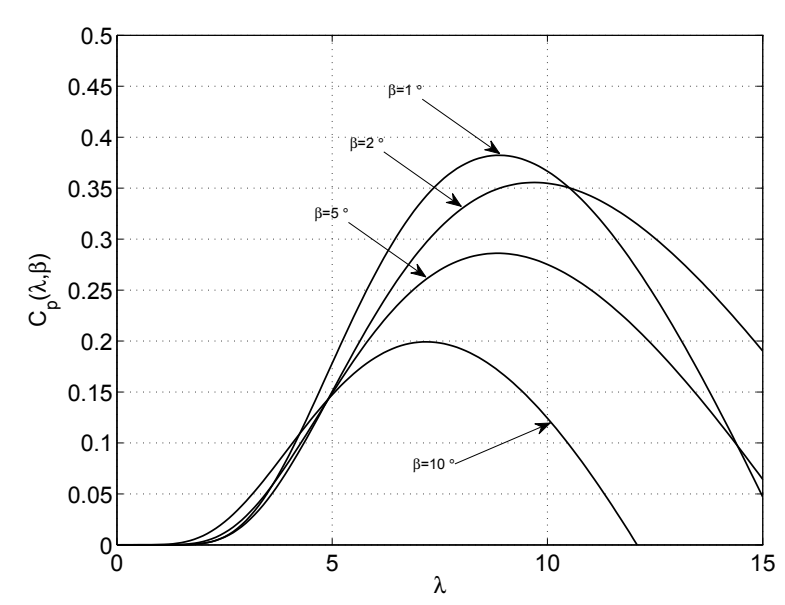

Figura 2.10: Aproximación analítica del coeficiente de potencia $\left(C_{p}(\lambda, \beta)\right)$.

Cuando el ángulo de paso de pala $\beta$ aumenta, el ángulo de ataque se reduce y por consiguiente el coeficiente de potencia $C_{p}(\lambda, \beta)$ se reduce. Esta característica se utiliza para limitar la velocidad de giro en algunas turbinas eólicas, como se comentó anteriormente. Si la variación del ángulo $\beta$ es positiva la estrategia para ajustar el valor de $C_{p}(\lambda, \beta)$ se denomina "to feather", mientras que si la variación es negativa se denomina "to stall".

La técnica de control del ángulo de la pala por pérdida o "stall" es más sensible a pequeñas variaciones del ángulo de pitch que reducen significativamente el valor del coeficiente de potencia $C_{p}(\lambda, \beta)$. El problema que presenta esta técnica es que induce mayores esfuerzos y las palas de la turbina están sometidas a más problemas de fatiga que si tuvieran paso fijo (Burton et al., 2011). La técnica conocida como pitch "to feather" necesita modificar más su ángulo para producir el mismo efecto de limitación de potencia que la técnica "to stall", reduciendo los efectos de las cargas aerodinámicas sobre la turbina. En condiciones con mucho viento, grandes excursiones del pitch de las palas es necesario para mantener la potencia constante (Burton et al., 2011). El rango de variación del ángulo de pitch usualmente va de unos pocos grados negativos hasta 30 grados.

Si bien el control de la pala por pérdida aerodinámica es una estrategia válida, en turbinas grandes preferentemente se utiliza el control por paso de pala "to feather" (Bianchi et al., 2006).

\subsubsection{Curva de potencia eléctrica de un aerogenerador}

La capacidad de generación de los aerogeneradores, se expresa por medio de la curva de potencia, la cual representa la potencia eléctrica en función de la velocidad del viento. En la Figura 2.11 se presenta una curva de potencia típica de un aerogenerador de 1,8 MW. Esta curva de potencia es obtenida por el fabricante del aerogenerador haciendo mediciones de la velocidad del viento y de la potencia generada por la máquina. En la misma se puede definir el rango de funcionamiento del aerogenerador en función de las siguientes velocidades de viento: 
- Velocidad de corte inferior, es la velocidad mínima en la cual el aerogenerador puede producir potencia útil.

- Velocidad de corte superior, es el punto en la velocidad del viento cuando se alcanza el límite de integridad de la máquina $(25 \mathrm{~m} / \mathrm{s}$ para el aerogenerador de la Figura 2.11) y se produce la desconexión.

Además, en la Figura 2.11 se indica el valor de la velocidad del viento donde se obtiene la potencia nominal.

El aerogenerador permanecerá apagado cuando la velocidad es demasiado baja para la producción de energía eléctrica. Para una velocidad de viento anual de $8 \mathrm{~m} / \mathrm{s}$ la velocidad de corte inferior puede ser de $5 \mathrm{~m} / \mathrm{s}$ (Olimpo et al., 2009). Cuando el viento supera el umbral de velocidad de corte inferior, el aerogenerador puede producir energía eléctrica. Desde el punto indicado en la Figura 2.11 como "Velocidad de corte inferior" hasta el valor de velocidad señalado como "Velocidad nominal" una máquina de velocidad variable es operada para maximizar la extracción de la energía del viento. Para la correcta elección de las velocidades

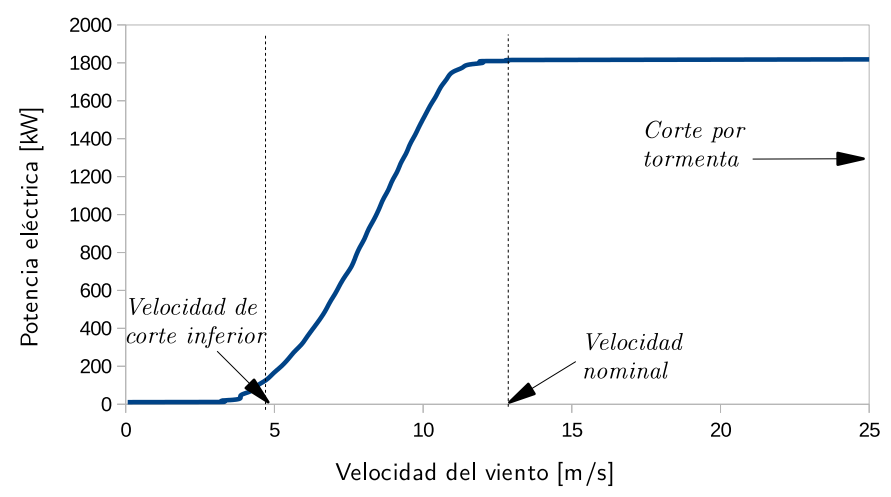

Figura 2.11: Curva de potencia típica de un aerogenerador de 1,8 MW.

de corte inferior y de corte por tormenta del aerogenerador, el diseñador debe balancear el recurso, la extracción de energía por parte del Aerogenerador y el costo de mantenimiento del mismo.

\subsubsection{Modelo de la turbina eólica}

La complejidad del modelo está relacionada con los problemas que se desean estudiar. En este sentido, pueden requerirse modelos dinámicos de distinta complejidad que incluyan:

- Las palas.

- El eje de baja velocidad.

- La caja multiplicadora.

- El eje de alta velocidad. 
Si el objetivo es estudiar el esfuerzo en las palas o en el tren de transmisión es necesario un modelo de varias masas. En este sentido, un mayor conocimiento sobre la dinámica del tren motriz de los aerogeneradores es de utilidad en el momento del diseño de los componentes internos de los sistemas de transmisión. En particular, las fallas debidas a problemas en las cajas de transmisión son responsables en más del $20 \%$ del tiempo de inactividad de los aerogeneradores (Girsang et al., 2013).

Por el contrario, para evaluar la interacción entre aerogeneradores y la red eléctrica puede emplearse un modelo de una masa como el que se presenta en las siguientes expresiones. En este modelo un eje rígido conecta el rotor de la turbina al generador, es decir no hay un desplazamiento angular en el eje. (Bustos et al., 2012).

La expresión dinámica del tren de potencia puede ser escrita del lado de la turbina como:

$$
\frac{d \Omega}{d t}=\frac{1}{J_{e q}^{\prime}}\left(T_{t}-T_{e m}^{\prime}\right),
$$

donde el par electromagnético equivalente está dado por:

$$
T_{e m}^{\prime}=k T_{e m},
$$

y el momento de inercia equivalente es:

$$
J_{e q}^{\prime}=k^{2} J_{g}+J_{T},
$$

donde $\Omega$ es la velocidad angular de la turbina, $T_{t}$ es el par aerodinámico de la turbina, $T_{e m}$ es el par electromagnético del generador, $J_{g}$ y $J_{T}$ son el momento de inercia del generador y de la turbina, respectivamente y $k$ es la relación de la caja multiplicadora.

\section{Modelo del control de pitch}

En general, el actuador de pitch es un servo no lineal que gira todas las palas a la vez o parte de ellas. En la Figura 2.12 se presenta un modelo de primer orden con saturación en la amplitud y la velocidad de paso de pala. El comportamiento dinámico del actuador operando en la zona lineal es capturado por la siguiente ecuación diferencial:

$$
\dot{\beta}=-\frac{1}{\tau} \beta+\frac{1}{\tau} \beta_{d},
$$

donde $\beta$ y $\beta_{d}$ es el valor actual y el valor deseado del pitch, respectivamente.

El rango típico del ángulo $\beta$ es de $-2^{\circ}$ a $30^{\circ}$ y la máxima tasa de variación es de $\pm 10^{\circ} / \mathrm{s}$ (Bianchi et al., 2006). 


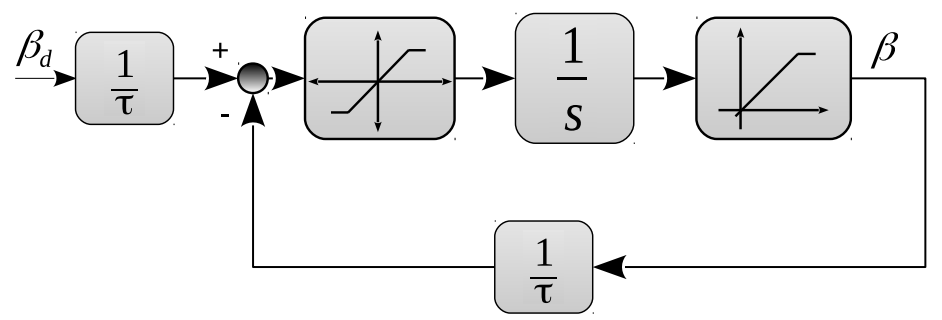

Figura 2.12: Modelo del actuador de pitch.

\subsection{Topologías de sistemas de conversión de energía eólica}

Una forma de clasificar a los aerogeneradores es considerando la configuración de conexión y el tipo de generador eléctrico utilizado. Otra posible clasificación puede realizarse según el modo en que los generadores son operados: a velocidad fija o a velocidad variable.

En las siguientes subsecciones se presentan las topologías más utilizadas y las ideas básicas de la electrónica de potencia asociada.

\subsubsection{Aerogeneradores de velocidad fija}

La Figura 2.13 muestra el esquema de un aerogenerador de velocidad fija con un generador asincrónico tipo jaula de ardilla.

Estos aerogeneradores giran a una velocidad casi constante, con una pequeña variación, del orden del $1 \%$ al $2 \%$. La velocidad es determinada por la relación de engranajes, la frecuencia de red y el número de polos del generador.

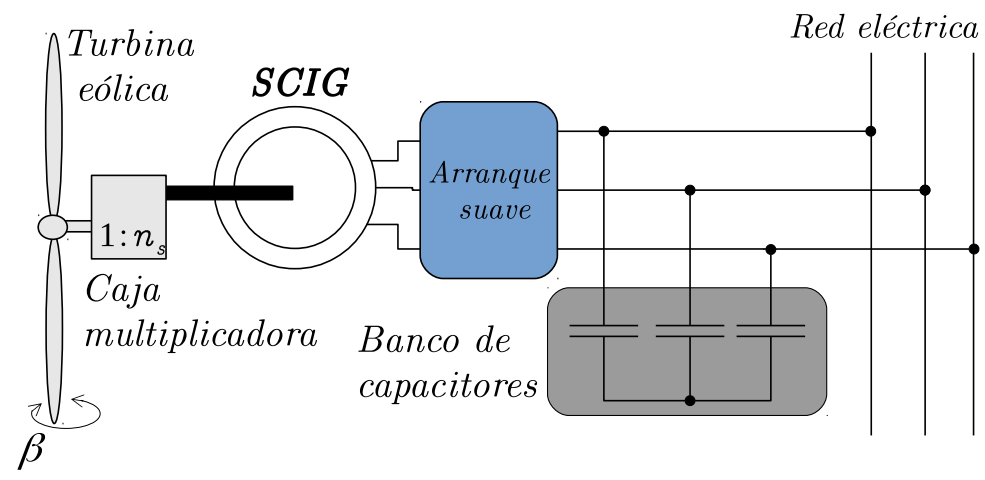

Figura 2.13: Esquema de aerogenerador de velocidad fija.

En general, este tipo de sistema de conversión de energía eólica emplea una turbina eólica con control de pitch "to stall" para limitar la potencia a velocidades de viento por encima de la nominal. El acople entre el generador y la turbina eólica es a través de una caja multiplicadora. La caja multiplicadora adapta las velocidades del eje de la turbina con la del generador. El generador se conecta de forma directa a la red y como los generadores de inducción de jaula de ardilla (SCIG, por su sigla en inglés) consumen potencia reactiva, para compensar esta potencia se coloca un banco de capacitores en cada aerogenerador (Olimpo et al., 2009). 
La conexión y desconexión del aerogenerador a la red eléctrica se lleva a cabo empleando un arranque suave. El arranque suave es utilizado para aumentar lentamente el flujo magnético, minimizando los transitorios de corriente cuando se energizan los arrollamientos del SCIG.

La máxima eficiencia de conversión puede ser lograda solo a una velocidad de viento. La eficiencia se degrada para otras velocidades de viento (Wu et al., 2011).

\subsubsection{Aerogeneradores de velocidad variable}

El concepto dominante detrás de aerogeneradores de gran escala son las máquinas de velocidad y pitch variable (Martin, 2010). Este concepto fue impulsado principalmente por los nuevos códigos de red para la conexión de granjas eólicas y por la reducción en la carga mecánica lograda con la operación a velocidad variable. La operación de los aerogeneradores a velocidad variable puede lograr la máxima eficiencia de conversión de energía para un gran rango de velocidades de viento.

La operación a velocidad variable se logra combinando generadores eléctricos (sincrónicos o asincrónicos) con una interfaz de electrónica de potencia (convertidor). Efectivamente, los aerogeneradores de velocidad variable pueden desacoplar la velocidad de rotación del rotor de la frecuencia de la red a través de convertidores electrónicos de potencia.

El principal inconveniente de estas topologías ha sido el costo del convertidor electrónico. Sin embargo, este costo adicional es compensado por el aumento en la eficiencia de conversión de energía. Los aerogeneradores de velocidad variable pueden producir alrededor de 8 a $10 \%$ más de potencia que los aerogeneradores de velocidad fija. Además, la operación a velocidad variable permite reducir los esfuerzos mecánicos sobre la turbina, el sistema de transmisión, la caja de engranajes (en caso de poseerla) y la estructura de la turbina eólica. En la tabla 2.1 se presentan las principales ventajas y desventajas de ambas arquitecturas en base a Wu et al. (2011).

Actualmente, las topologías más comunes que operan a velocidad variable emplean:

- Aerogeneradores equipados con generadores de inducción asincrónico doblemente alimentados, (DFIG, por su sigla en inglés) conectados a red por estator con un convertidor electrónico de potencia espalda contra espalda en el circuito del rotor.

- Aerogeneradores basados en generadores sincrónicos con convertidores electrónicos de potencia entre el estator y la red. En este caso el convertidor procesa el flujo completo de potencia.

Un aerogenerador que emplea un generador de inducción asincrónico doblemente alimentado (DFIG) se muestra en la Figura 2.14. El generador DFIG está constituido por un rotor bobinado con anillos rozantes para permitir la circulación de la corriente de forma bidireccional al bobinado rotórico de la máquina. El rotor del generador está conectado a la red a través de convertidores que controlan las corrientes del rotor, desacoplando la velocidad del rotor de la frecuencia de red. El control electrónico por rotor permite el manejo independiente de las potencias activas y reactivas.

Un DFIG puede inyectar potencia a través del estator y del rotor. El rotor puede absorber 
Tabla 2.1: Ventajas y desventajas de los aerogeneradores de velocidad fija y variable.

\begin{tabular}{|l|l|l|}
\hline Velocidad fija & \multicolumn{1}{|c|}{ Ventajas } & \multicolumn{1}{|c|}{ Desventajas } \\
\hline & $\begin{array}{l}\text { Bajo costo de operación } \\
\text { y de mantenimiento. }\end{array}$ & $\begin{array}{l}\text { Stress mecánico elevado. } \\
\text { de energía eléctrica. }\end{array}$ \\
\hline & Velocidad Variable & Stress mecánico reducido. \\
& $\begin{array}{l}\text { Costo adicional debido a } \\
\text { los convertidores de potencia. }\end{array}$ \\
\hline & de conversión de energía. & Sistema de control más complejo. \\
\hline & $\begin{array}{l}\text { Mejora de la calidad de } \\
\text { la energía eléctrica. }\end{array}$ & \\
\hline & Controlabilidad. & \\
\hline & Verifica los códigos de red. & \\
\hline
\end{tabular}

o entregar potencia dependiendo de su velocidad. Si la velocidad del rotor está por encima de la velocidad sincrónica, el DFIG entrega potencia a través de los convertidores conectados al rotor de la máquina. A su vez el rotor, operando por debajo de la velocidad sincrónica, absorbe potencia de la red eléctrica mediante los convertidores conectados al rotor.

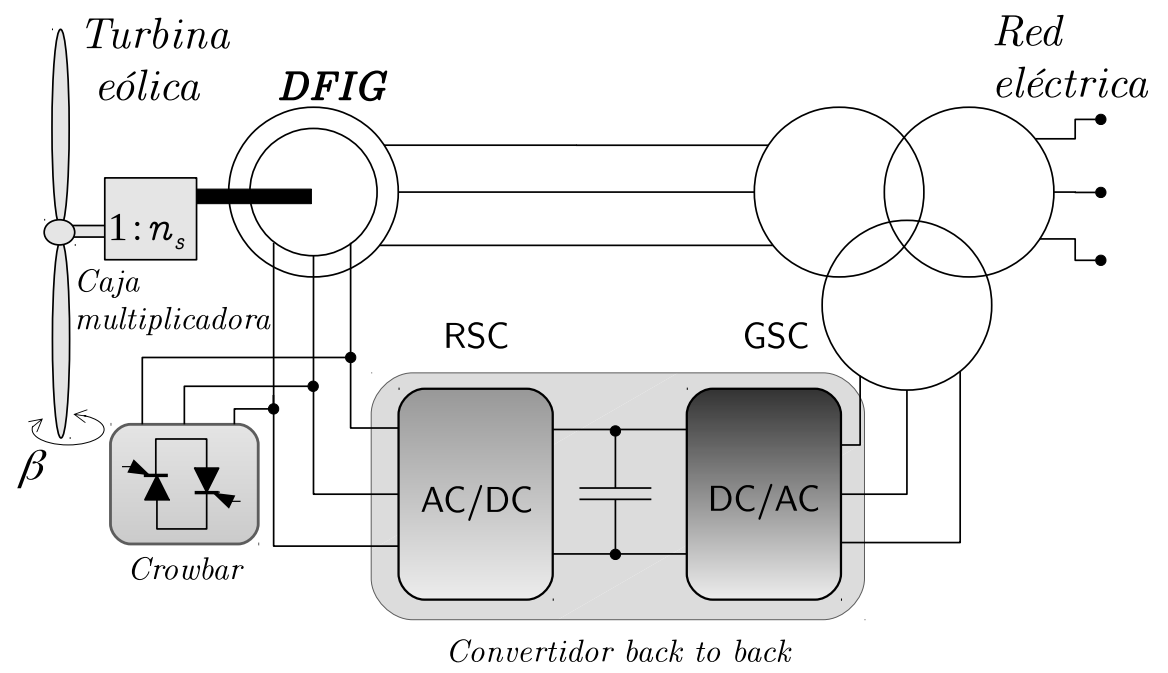

Figura 2.14: Esquema de aerogenerador equipado con un DFIG.

Para proteger los convertidores de potencia de sobretensiones y sobrecorrientes, los generadores DFIG suelen estar equipados con un dispositivo conocido como "crowbar", el cual 
cortocircuita los terminales del bobinado del rotor frente a una falla. Los "crowbars" pueden ser implementados utilizando bancos de tiristores en antiparalelo o empleando transistores IGBT (Olimpo et al., 2009).

En general, el objetivo del convertidor del lado del rotor (RSC, por su sigla en inglés "Rotor Side Converter"), es la de controlar independientemente la potencia activa y la reactiva del generador. Mientras que el objetivo del convertidor del lado de la red (GSC, por su sigla en inglés "Grid Side Converter") es la de mantener constante la tensión en el bus de continua para garantizar el flujo de potencia (Hansen et al., 2006). Adicionalmente, el convertidor del lado de la red puede ser controlado para contribuir con potencia reactiva a la red.

Con respecto a los esquemas con un convertidor completo en el estator, la configuración con un DFIG emplea un convertidor que solo maneja del 25 al $30 \%$ de la potencia eléctrica generada. Esto se ve reflejado en el menor costo del filtro de salida y de la electrónica de potencia asociada al convertidor espalda contra espalda (Ezzat et al., 2013).

Otra configuración típica de un aerogenerador de velocidad variable es presentado en la Figura 2.15. Este tipo de configuración emplea un convertidor de potencia bidireccional que maneja toda la potencia. Además, es posible utilizar diferentes tipos de generadores.

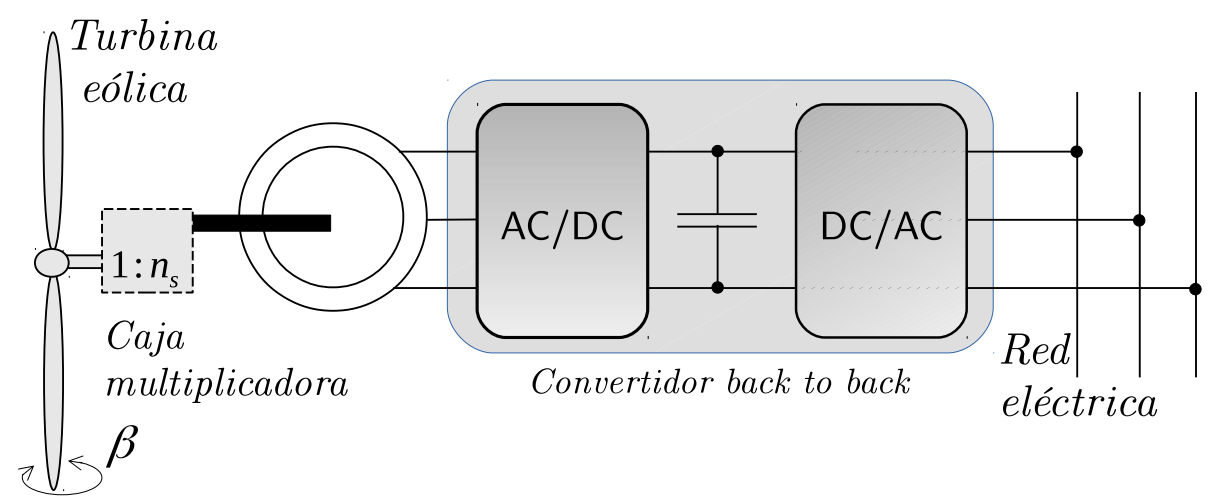

Figura 2.15: Esquema de un aerogenerador con un convertidor completo.

La dinámica de operación del generador es aislada de la red eléctrica en el sentido que la velocidad del rotor del generador puede ser controlada independientemente de la frecuencia de la red. Esto permite la operación del aerogenerador a velocidad variable.

\subsection{Requerimientos de red para la integración de energía eólica}

Los códigos de red definen los requerimientos de conexión de sistemas de generación de energía eléctrica y cargas a una red eléctrica para asegurar eficiencia, calidad, seguridad y operación económica de los sistemas de distribución y transmisión.

En muchos casos, estas disposiciones exigen a las estaciones de generación eólica características operativas similares a las centrales eléctricas convencionales.

Los requerimientos de red difieren de país en país y también pueden diferir entre 
regiones. Estas pueden tener características comunes pero algunos requerimientos pueden ser sustancialmente diferentes, reflejando la particularidad de las redes individuales. Como otro requisito, los niveles y períodos de tiempo de la salida de potencia de una planta de generación deben ser mantenidos en valores especificados.

Una forma en que los requerimientos suelen ser definidos se observa en la Figura 2.16. Donde se señala la zona en la que debería operar el sistema de forma continua y con recuadros sombreados las zonas en donde se puede operar por un corto período de tiempo.

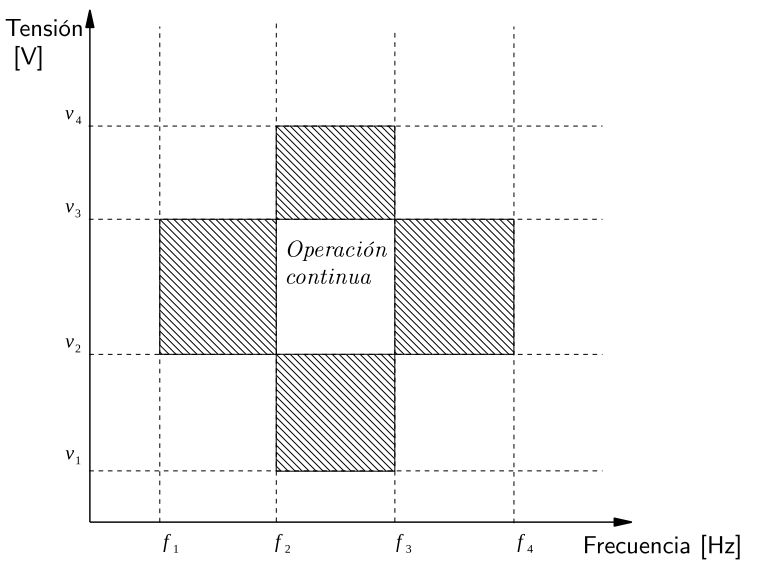

Figura 2.16: Forma típica de expresar requerimientos de operación continua y reducida.

Las reglas de interconexión para las granjas están siendo reformuladas en paralelo con el incremento de la penetración y el desarrollo de tecnología. Entre otros requisitos vinculados a distintos aspectos de la conexión de las granjas eólicas al sistema eléctrico, pueden citarse (Ackermann, 2012):

- Control de potencia activa: aunque con diferentes objetivos, varios códigos de red requieren del control de potencia activa de las granjas.

- Control de frecuencia: contribuir a mantener la frecuencia del sistema dentro de límites aceptables garantiza la seguridad del servicio, se previene la sobrecarga del equipamiento y se satisfacen los estándares de calidad.

- Rangos de frecuencia y de tensión: se requiere que las granjas eólicas se mantengan en operación aún cuando los valores de frecuencia y tensión se encuentren alejados de sus valores nominales.

- Calidad de tensión (flicker, armónicas, cambios rápidos): diversos requerimientos según los distintos países.

- Transformadores tipo "tap changing": algunos códigos los requieren en caso de necesitar el cambio de la relación de transformación entre la granja eólica y la red.

- Protección de las granjas eólicas: considera perturbaciones en la red. Un sistema de protección tipo relé debe actuar en presencia de corrientes de cortocircuito, caídas de tensión, sobretensiones, durante y después de una falla. Esto debe asegurar que la granja obedezca a requisitos para la operación de la red y la apoye durante y después de una falla, por ejemplo debe permanecer conectada durante fuertes fallas de tensión (FRT, por su sigla en inglés, Fault ride-through). 
- Modelado y verificación de una granja eólica: algunos códigos requieren los modelos de las granjas eólicas a los efectos de permitir al operador investigar por simulaciones la interacción entre la granja y el sistema de potencia. Además, se puede requerir de la instalación y monitoreo de diversas variables para verificar el comportamiento real durante fallas y para verificar el modelo.

- Comunicaciones y control externo.

Adicionalmente existen requerimientos para salvaguardar a los aerogeneradores de daños provenientes de defectos propios o de su instalación y contra perturbaciones exteriores como:

- corrientes de cortocircuito entre fases y tierra;

- bajas tensiones por recuperación de cortocircuitos;

- incrementos de tensión por fallas trifásicas y de una fase a tierra;

- falta de fase;

- recierre por falta de fase y otros impactos producto de operaciones anormales.

\subsubsection{Control de potencia activa y respuesta a cambios de frecuencia}

Para que las granjas eólicas puedan participar activamente en el control primario y secundario, con capacidad de respuesta ante cambios en la frecuencia y la limitación de las velocidades de cambio de la potencia activa generada, los requerimientos pueden incluir (Tsili y Papathanassiou, 2009):

1. Control de potencia: la producción de la granja es ajustada a un valor especifico dentro de las limitaciones que impone el recurso renovable. En la Figura 2.17 a) se observa este concepto.

2. Control delta: la granja puede operar con una reserva constante de potencia activa en MW o con un porcentaje de la potencia disponible de manera de disponer de esa reserva para actuar cuando se modifique la frecuencia Figura $2.17 \mathrm{~b}$ ).

3. Limitación de gradiente: se limita la velocidad con que la potencia activa de la granja puede ser cambiada de manera de no perturbar (desbalanceando) el equilibrio de potencias con el resto de la red. Así, se buscan limitar los cambios de frecuencia asociados a variaciones extremas causadas por cortes o arranques de toda una central eólica Figura 2.17 c).

4. Respuesta a cambios de frecuencia: es la capacidad de las granjas de variar la potencia activa generada frente a cambios en la frecuencia.

\subsubsection{Regulación de potencia reactiva y de tensión}

Los requerimientos de control de la potencia reactiva dependen de las características constitutivas de cada red eléctrica. Es decir, para redes con valores diferentes de impedancia 

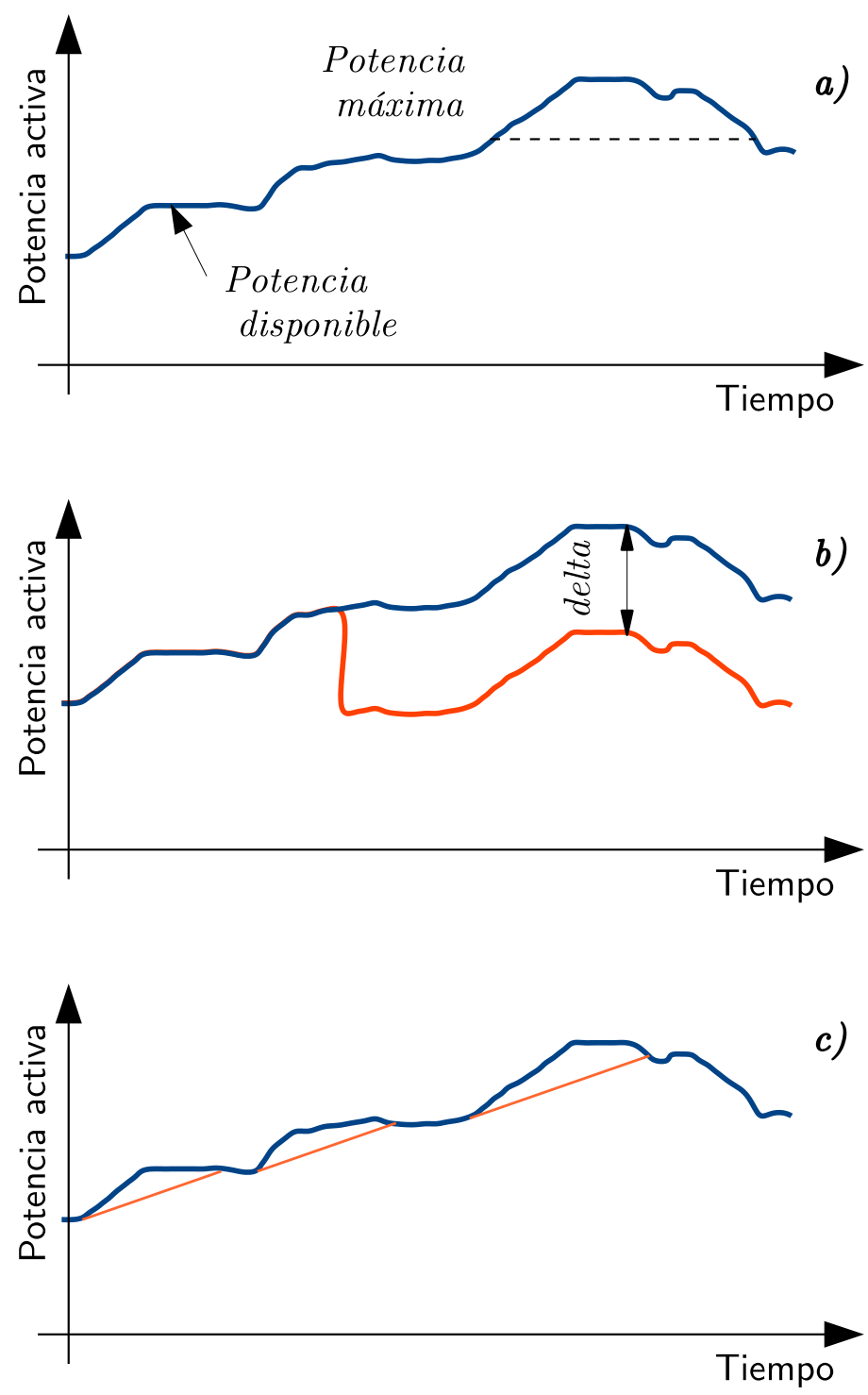

Figura 2.17: Especificaciones de control de potencia, delta y gradiente.

la misma inyección de potencia reactiva puede producir diferentes incrementos en la tensión (Di Marzio et al., 2007). En general, los requisitos de los códigos para la potencia reactiva se extienden desde los factores de potencia 0.925 en adelanto a los 0.85 en atraso. Con respecto a la tensión, en algunos códigos de red se está comenzando a solicitar, que las granjas controlen la tensión en el punto de la conexión a un valor de referencia predeterminado, definido por el operador de la red de transmisión.

\subsubsection{Rango de frecuencias de funcionamiento}

Desviaciones de la potencia generada o consumida respecto de las previsiones corren la frecuencia fuera de los valores deseados. Un incremento anormal en la frecuencia aumenta las temperaturas de operación de los generadores disminuyendo la vida útil de las aislaciones a la vez que puede dañar al equipamiento electrónico. Antiguamente, los operadores de la red eléctrica requerían la desconexión de las granjas eólicas en el caso de una caída de frecuencia, 
no obstante si grandes cantidades de energía eólica se desconectan se afecta la habilidad del sistema para recuperar su estado normal. Los operadores de red definen rangos de frecuencias y duración permitida. También algunos operadores de red definen tasas de cambio de la frecuencia (ROCOF, rate of change of frequency) (Mokui et al., 2014).

En la Figura 2.18 se observan los rangos de funcionamiento para una granja eólica conectada a una red eléctrica para diferentes países (Tsili y Papathanassiou, 2009).

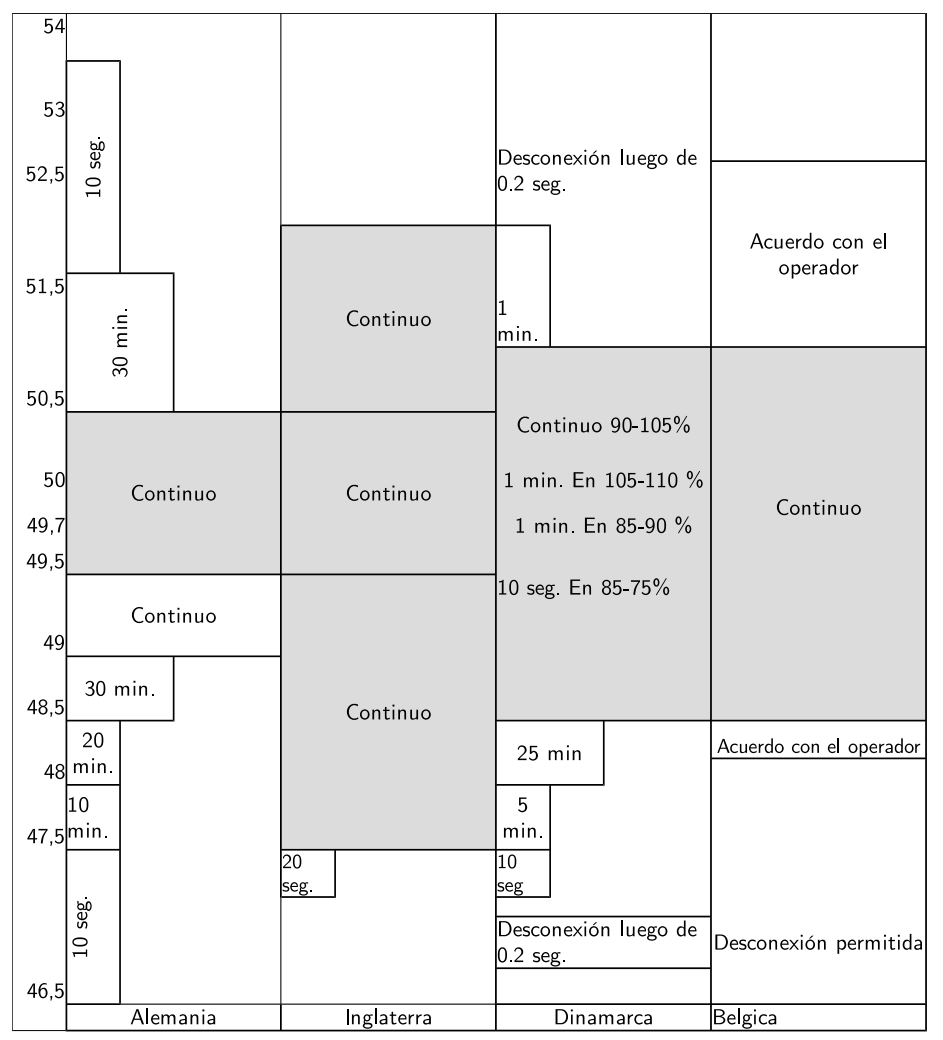

Figura 2.18: Rango de frecuencias de operación para diferentes operadores.

\subsubsection{Capacidad para manejar una falla (FRT)}

La ocurrencia de un falla en algún punto de la red eléctrica puede resultar en un hueco de tensión en una o más fases. Dependiendo del tipo y la localización de la falla ésta puede ser propagada en toda la red, especialmente en el caso de redes débiles. Además, con el incremento de la penetración de parques eólicos conectados a redes de transmisión es de utilidad que los sistemas de generación puedan seguir funcionando ante una falla en la red eléctrica. Estas capacidades son conocidas como capacidad para manejar la falla. Las especificaciones para manejar una falla (FRT, por su sigla en ) suelen indicarse como en la Figura 2.19. En esta figura se definen dos zonas de operación, indicadas como "a)" y "b)". En el caso en que la tensión en el punto de conexión se encuentre en la zona "a)" los aerogeneradores que componen la granja eólica deben permanecer conectados. Mientras, que si la tensión se encuentra en la zona por debajo de la curva, es decir en la zona "b)", los aerogeneradores se pueden desconectar de la red. 


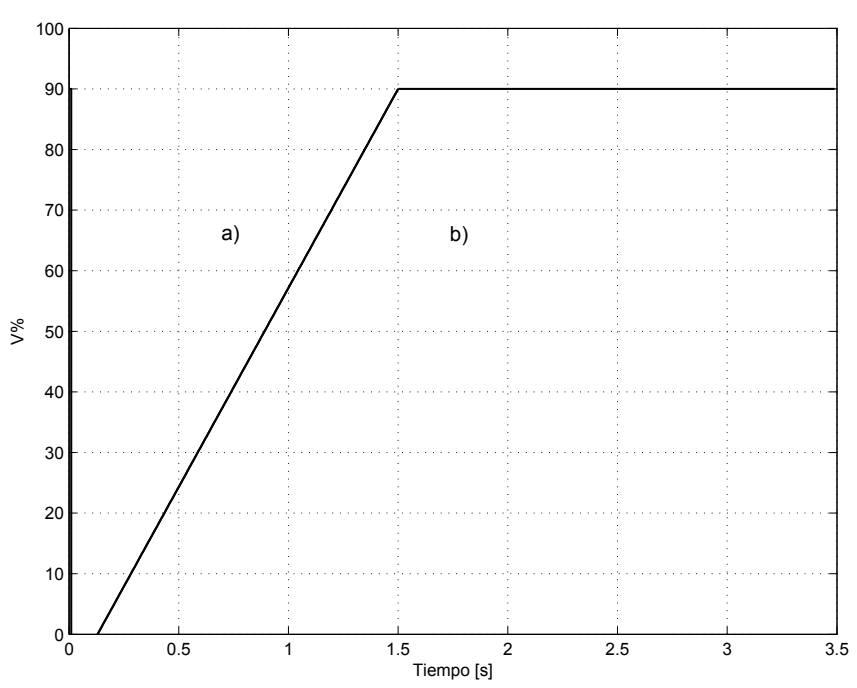

Figura 2.19: Límite para un requerimiento de FRT .

La especificación del hueco es dependiente del tiempo de respuesta de las protecciones y puede variar de una décima de segundo a algunos segundos (Tsili y Papathanassiou, 2009). Algunas regulaciones prevén que las granjas eólicas deben dar soporte a la red eléctrica en caso de una falla, como una caída de tensión. Esto se realiza inyectando una potencia reactiva en el punto de conexión para tratar de mantener y restaurar la tensión en el PCC rápidamente.

\subsection{Retrospección}

En este capítulo se presentaron características sobresalientes de la energía eólica a los efectos de poder obtener un mejor entendimiento del recurso renovable.

Se comentó acerca de la existencia de un límite teórico, conocido como límite de Betz, para la extracción de energía por parte de una turbina eólica de eje horizontal.

Se presentaron las ecuaciones de energía y potencia eólica disponible. También se presentaron las expresiones del par y la potencia de un aerogenerador de eje horizontal.

Se presentó el modelo mecánico de la turbina y un modelo del actuador de pitch.

Se presentó una clasificación genérica para los aerogeneradores en función de las máquinas utilizadas.

A pesar que hace unos años atrás el control de una granja estaba diseñado para solo obtener la máxima potencia disponible del viento, se requiere que hoy la granja eólica sea vista por la red como una planta de generación equipada con generadores sincrónicos. 


\section{Capítulo 3}

\section{REDES}

\section{Resumen}

En este capítulo se presentan las herramientas básicas para el análisis de una red eléctrica. Se exponen las expresiones para la potencia activa y la potencia reactiva de un sistema eléctrico de potencia.

Se presentan los diferentes tipos de buses considerados en un sistema de transmisión de energía.

Se presentan los modelos estáticos y dinámicos de las cargas de un sistema de potencia. Estos modelos representan a la carga a través de la potencia activa y reactiva que ésta consume en función de las variables del sistema.

Se presentan las ecuaciones que describen el comportamiento del generador sincrónico a través de las ecuaciones de oscilación o swing.

También se presentan las diferentes tecnologías referidas a sistemas de almacenamiento de energía aplicados a sistemas de generación de energía renovable. Estas tecnologías presentan beneficios en el sentido que mejoran la estabilidad, la calidad y la confiabilidad de la energía eléctrica.

Finalmente, se introducen las ideas básicas de una micro red.

\subsection{Redes}

Los elementos principales de una red eléctrica son generadores, transformadores, líneas de transmisión y distribución, cargas eléctricas y sistemas de protección y control. Una red eléctrica de potencia debe satisfacer la demanda de energía eléctrica con una buena calidad de la energía. La calidad del servicio eléctrico puede ser medida en función de frecuencia constante, factor de potencia, fases balanceadas, formas de onda sinusoidales, bajo contenido armónico, etc. 
Tradicionalmente una red eléctrica de potencia es dividida en 4 partes llamadas generación, transmisión, distribución y utilización. La red de transmisión opera en los niveles de alta tensión, en el orden de $66 \mathrm{kV}$ a $220 \mathrm{kV}$, permitiendo mayor eficiencia para transportar grandes cantidades de energía (Hacha, 2002). La tensión en una línea de distribución varía entre los $220 \mathrm{~V}$ y los $66 \mathrm{kV}$. Los sistemas de distribución se dividen en sistemas de distribución de media tensión y de baja tensión. Los sistemas de baja tensión o de utilización manejan tensiones de 50-1000 $V$ y los sistemas de distribución de media tensión de $1 \mathrm{kV}$ a $66 \mathrm{kV}$.

\subsubsection{Líneas de transmisión de AC}

En esta subsección se describen los factores que influyen en la transferencia de potencia activa y reactiva de un sistema. Con este objetivo, se considera el sistema de la Figura 3.1 compuesto por dos fuentes interconectadas por un sistema de transmisión inductivo (Kundur, 1993).

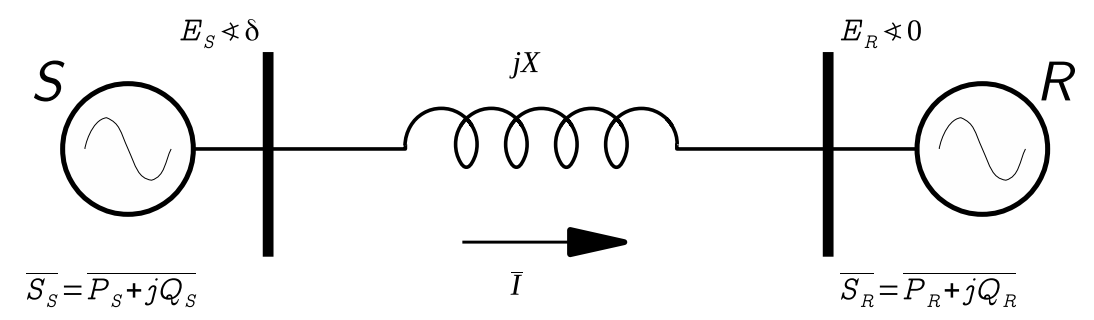

Figura 3.1: Diagrama equivalente del sistema de potencia.

En la Figura 3.2 se presenta un diagrama fasorial del sistema analizado, donde $E_{S}$ y $E_{R}$ son los módulos de la tensión en las fuentes "S" y "R", respectivamente, $\delta$ es el ángulo de la carga y $\phi$ es el ángulo del factor de potencia.

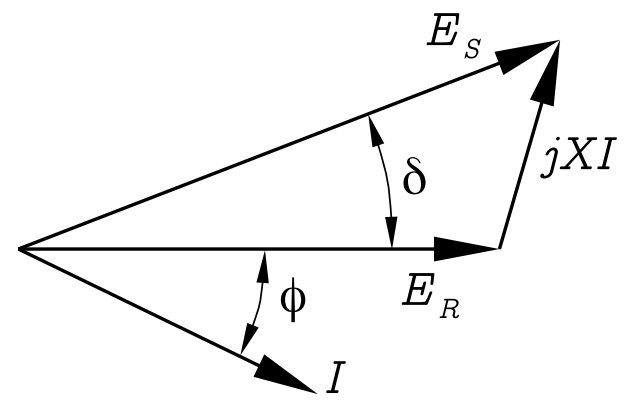

Figura 3.2: Diagrama fasorial.

A partir del análisis de la Figura 3.1 es posible escribir la potencia compleja en el nodo $R$ como:

$$
\bar{S}_{R}=P_{R}+j Q_{R}=\bar{E}_{R} \bar{I}^{*} \Rightarrow \bar{E}_{R}\left[\frac{\bar{E}_{S}-\bar{E}_{R}}{j X}\right]^{*} .
$$

La tensión en el nodo $S$, puede escribirse como:

$$
\bar{E}_{S}=E_{S}(\cos (\delta)+j \operatorname{sen}(\delta)) .
$$


Reemplazando la expresión anterior de la tensión en el nodo $S$ en la ecuación (3.1) se obtiene

$$
\bar{S}_{R}=E_{R}\left[\frac{E_{S}(\cos (\delta)+j \operatorname{sen}(\delta))-E_{R}}{j X}\right]^{*}
$$

A partir de la ecuación de la potencia compleja anterior es posible obtener las expresiones de flujo de potencia activa y reactiva en un sistema de potencia. La potencia activa en el nodo $R$ es:

$$
P_{R}=\frac{E_{R} E_{S}}{X} \operatorname{sen}(\delta),
$$

mientras que la parte imaginaria, (potencia reactiva), de la potencia compleja es:

$$
Q_{R}=\frac{E_{R} E_{S} \cos (\delta)-E_{R}^{2}}{X} .
$$

De manera similar se pueden obtener las expresiones para la potencia activa y reactiva en el nodo "S", de la siguiente manera:

$$
P_{S}=\frac{E_{S} E_{R}}{X} \operatorname{sen}(\delta)
$$

y

$$
Q_{S}=\frac{E_{S}^{2}-E_{S} E_{R} \cos (\delta)}{X} .
$$

Note la simetría en esta última expresión y la ecuación de $Q_{R}$ (3.5). Las ecuaciones (3.4), (3.5), (3.6) y (3.7) describen la forma en que se realiza el intercambio de potencia activa y potencia reactiva entre dos nodos de un sistema de potencia. Si se considera $\delta=0$ el término para la potencia activa es $P_{r}=0$ y la expresión para la potencia reactiva es:

$$
Q_{R}=\frac{E_{R} E_{S}-E_{R}^{2}}{X} .
$$

Suponiendo que el valor de la tensión en la fuente "S" es mayor a la fuente "R", $\left(E_{S}>E_{R}\right)$. Entonces en la expresión (3.8) se puede observar que $E_{R} E_{S}>E_{R}^{2}$, por lo tanto $Q_{R}>0$. La potencia reactiva fluye de la fuentes "S" a la fuente "R". En el caso que $E_{R}>E_{S}$ se presenta la situación inversa, la potencia circula de la fuente "R" a "S". De esta forma se puede observar que en un sistema como el de la Figura $3.1 \operatorname{con} \delta=0$ para transmitir potencia reactiva de un punto a otro uno de ellos debe tener una tensión mayor (Kundur, 1993).

Considerando que el módulo de las dos tensiones en las dos fuentes son iguales, es decir $E_{S}=E_{R}=E$ y $\delta \neq 0$. Las expresiones para la potencia activa y reactiva en la fuente "R" son:

$$
\begin{gathered}
P_{R}=\frac{E^{2}}{X} \operatorname{sen}(\delta), \\
-Q_{S}=Q_{R}=\frac{E^{2}}{X}(\cos (\delta)-1) .
\end{gathered}
$$

Para la potencia activa, cuando $\delta>0 \Rightarrow P_{R}>0$, la potencia activa fluye de la fuente "S" a la fuente "R". Si $\delta<0$ la potencia activa fluye de la fuente "R" a "S". Mientras que el consumo de la línea de transmisión es provisto por las dos fuentes "S" y "R".

La transferencia de potencia reactiva depende básicamente de la diferencia de las magnitudes de tensión entre los nodos. De esta forma es posible observar que la potencia 
reactiva no puede ser transportada por grandes distancias debido a la diferencia de tensión asociada. Se puede observar en la expresión (3.9) que la potencia activa es función de la tensión y del ángulo $(\delta)$. Sin embargo, en un sistema de potencia se intenta que la tensión sea constante, por lo tanto para modificar el flujo de potencia activa entre dos fuentes se prefiere variar fundamentalmente el ángulo $\delta$.

\subsubsection{Clasificación de los diferentes tipos de buses}

Las definiciones de un bus de potencia son utilizadas para el cálculo del flujo de potencia. $\mathrm{Si}$ el sistema se asume balanceado, es posible representar el sistema con una sola fase. Las variables primarias para analizar el problema del flujo de potencia son: la potencia activa en la red, la potencia reactiva, la magnitud de la tensión y el ángulo de la tensión con respecto a un punto de referencia.

Los siguientes tipos de buses o nodos son representados por dos de las cuatro cantidades.

- P-V, la potencia activa y la magnitud de la tensión son especificados. Estos valores por ejemplo pueden ser controlados por el control de velocidad y la excitación de una máquina sincrónica.

- P-Q, las potencias activa y reactiva son especificadas. El bus P-Q también es conocido como barras de carga. Generalmente las cargas son para lograr potencia constante.

- Slack, Swing u oscilantes, la magnitud de la tensión y la fase del ángulo son especificadas.

En la Tabla 3.1 se resumen las característica de los buses.

Tabla 3.1: Tipos de buses

\begin{tabular}{|l|l|l|l|l|}
\hline & $\mathbf{P}$ & $\mathbf{Q}$ & V & delta \\
\hline \hline Bus P-V & conocido & desconocido & conocido & desconocido \\
\hline Bus P-Q & conocido & conocido & desconocido & desconocido \\
\hline Bus Slack & desconocido & desconocido & conocido & conocido \\
\hline
\end{tabular}

\subsubsection{Ecuaciones de redes}

En está subsección resumimos las expresiones de nodos y las expresiones de potencia de un sistema eléctrico de potencia para su análisis.

\section{Ecuaciones de nodos}

Las ecuaciones de nodos son preferidas porque el número de ecuaciones de nodos es más pequeño que el número de ecuaciones de lazos independientes. Las ecuaciones de red en 
términos de la matriz de admitancia puede ser escrita como sigue:

$$
\left[\begin{array}{c}
\bar{I}_{1} \\
\bar{I}_{2} \\
\cdots \\
\bar{I}_{n}
\end{array}\right]=\left[\begin{array}{cccc}
Y_{11} & Y_{12} & \cdots & Y_{1 n} \\
Y_{21} & Y_{22} & \cdots & Y_{2 n} \\
\cdots & \cdots & \cdots & \cdots \\
Y_{n 1} & Y_{n 2} & \cdots & Y_{n n}
\end{array}\right]\left[\begin{array}{c}
\bar{V}_{1} \\
\bar{V}_{2} \\
\cdots \\
\bar{V}_{n}
\end{array}\right],
$$

donde $n$ es el número total de nodos, $Y_{i i}$ es la admitancia en el nodo $i$ (es decir, la suma de todas las admitancias en el nodo $i$ ), $Y_{i j}$, es la admitancia mutua entre los nodos $i$ y $j$ (o sea, es la suma negativa de todas las admitancias entre los nodos $i-j), \bar{V}_{i}$ es el fasor de tensión con respecto a tierra en el nodo $i$ y $\bar{I}_{i}$ es el fasor de corriente en el nodo $i$.

\section{Ecuaciones de potencias}

Además de la descripción empleando las ecuaciones de nodo, una red eléctrica puede ser analizada mediante las potencias activa y reactiva.

La expresión de la potencia compleja en el nodo $k$ puede desarrollarse como:

$$
\bar{S}_{k}=P_{k}+j Q_{k}=\bar{V}_{k} \bar{I}_{k}^{*} .
$$

Las ecuaciones de las potencias son de utilidad tanto en el análisis de estado estacionario como en el análisis de estabilidad del sistema ya que capturan la dinámica de la red en términos de potencia.

La corriente en el nodo $k$ puede desarrollarse como:

$$
\bar{I}_{k}=\sum_{m=1}^{n} Y_{k m} V_{m}
$$

donde la admitancia en el nodo $k$ está dada por:

$$
Y_{k m}=G_{k m}+j B_{k m}
$$

Si en la expresión (3.12) se reemplaza la definición de la admitancia de la expresión (3.14) se obtiene:

$$
P_{k}+j Q_{k}=V_{k} \sum_{m=1}^{n}\left(G_{k m}-j B_{k m}\right) \bar{V}_{m}^{*},
$$

El producto de los fasores $\bar{V}_{k}$ y $\bar{V}_{m}^{*}$ puede ser expresado como:

$$
\bar{V}_{k} \bar{V}_{m}^{*}=\left(V_{k} e^{j \theta_{k}}\right) V_{m} e^{-j \theta_{m}} \Rightarrow V_{k} V_{m} e^{j\left(\theta_{k m}\right)},
$$

donde $\theta_{k m}=\theta_{k}-\theta_{m}$ y aplicando la relación de Euler, la expresión anterior queda como:

$$
\bar{V}_{k} \bar{V}_{m}^{*}=V_{k} V_{m}\left(\cos \left(\theta_{k m}\right)+j \operatorname{sen}\left(\theta_{k m}\right)\right) .
$$

De esta manera las expresiones para la potencia activa y la potencia reactiva son:

$$
\begin{aligned}
& P_{n k}=V_{k} \sum_{m=1}^{n}\left(G_{k m} V_{m} \cos \left(\theta_{k m}\right)+B_{k m} V_{m} \operatorname{sen}\left(\theta_{k m}\right)\right), \\
& Q_{n k}=V_{k} \sum_{m=1}^{n}\left(G_{k m} V_{m} \operatorname{sen}\left(\theta_{k m}\right)-B_{k m} V_{m} \cos \left(\theta_{k m}\right)\right),
\end{aligned}
$$


donde $V_{k}$ es la tensión en el nodo genérico $k, G_{k}$ es la conductancia y $B_{k}$ es la susceptancia en el nodo $k$ de los dispositivos que componen la red eléctrica.

Las ecuaciones anteriores, (3.18) y (3.19), describen las potencias activa y reactiva en un nodo en función del módulo de la tensión y el ángulo $\theta$ de todos los buses.

Las ecuaciones (3.18) y (3.19) pueden ser utilizadas como una herramienta para evaluar el comportamiento de estado estacionario de una red eléctrica de potencia. Estas ecuaciones son no lineales y pueden ser resueltas empleando un método numérico. El método NewtonRaphson es utilizado eficientemente para resolver las expresiones (3.18) y (3.19). Dos variables son especificadas, según el tipo de bus ( P-V, P-Q, Slack), y las otras dos variables son obtenidas a través del método numérico con la precisión deseada (Hacha, 2002).

\subsubsection{Redes débiles}

El término red débil o fuerte es utilizado para caracterizar un nodo de una red eléctrica de potencia. De forma cualitativa, un nodo es débil cuando cambios en el flujo de las potencias activa y reactiva pueden causar variaciones significativas en la frecuencia o la tensión en el nodo analizado. Estas variaciones a su vez pueden afectar a los nodos vecinos ya que están interconectados por una línea. Es necesario remarcar que existen redes que pueden ser fuertes en frecuencia pero débiles en tensión y viceversa. Debido a que las redes en las zonas rurales están alejadas de las centrales de generación y las impedancias asociadas a las redes no son despreciables, éstas por lo general son más débiles que en las redes de las zonas urbanas o industriales. Por otra parte, el término débil también está referido a que en el nodo en cuestión existe una baja potencia de corto circuito (Tande, 2000).

La relación entre la potencia de cortocircuito y la potencia activa en el punto común de conexión (PCC) de una unidad de generación de energía eléctrica da una idea de que tan débil es la red y está dada por la siguiente relación:

$$
r=\frac{S_{s c}}{P}
$$

donde $S_{s c}$ es la potencia de cortocircuito en el nodo y $P$ es la potencia activa de la unidad de generación conectada al nodo. Los valores $r<20$ corresponden a una red débil (Farias et al., 2010).

\subsection{Cargas estáticas y dinámicas}

Las características de la carga tienen un rol importante en el análisis de estabilidad de un sistema de potencia. El modelo de una carga utilizada en estudios de estabilidad debe considerar un gran número de dispositivos como lámparas, motores de inducción, etc. La precisión del modelo de la carga tiene un impacto significativo en el análisis de estabilidad y control del sistema de potencia (Ma et al., 2006). Los modelos de cargas son tradicionalmente clasificados en modelos estáticos y modelos dinámicos (Kundur, 1993). Un modelo estático de una carga es representado como una función dependiente de la tensión o la frecuencia. Mientras que el modelo dinámico de la demanda de carga esta compuesto con una variable adicional que es el tiempo (Kundur, 1993; Song et al., 2013). 


\subsubsection{Cargas estáticas}

Un modelo estático de la carga describe la relación entre una tensión o frecuencia en el bus y la carga (potencia activa y reactiva). Generalmente, existen dos modelos para representar una carga estática, el modelo exponencial y el modelo ZIP.

El modelo exponencial para la carga describe la relación de la potencia activa y reactiva con la tensión en el punto de conexión con una función exponencial. La carga se puede representar escribiendo las expresiones para la potencia activa y reactiva de forma separada, es decir:

$$
\begin{aligned}
& P=P_{0}(\bar{V})^{a}, \\
& Q=Q_{0}(\bar{V})^{b},
\end{aligned}
$$

donde, $\bar{V}=\frac{V}{V_{0}}, P$ y $Q$ son las componentes de la potencia activa y reactiva de la carga. $V$ es la tensión en el bus, $P_{0}, Q_{0}$ y $V_{0}$ son la potencia activa, reactiva y la tensión en la condición de operación inicial. Los valores " $a$ " y " $b$ " son próximos a las pendiente $\frac{d p}{d v}$ y $\frac{d q}{d v}$ en $V=V_{0}$, respectivamente. Los valores típicos para los parámetros $a$ y $b$ son:

$$
0,5 \leq a \leq 1,8
$$

y

$$
1,5 \leq b \leq 6
$$

Una carga puede ser representada por un modelo polinómico dependiente de la tensión, conocido como ZIP. Dicho modelo es una representación polinómica en el que cada elemento varía de forma cuadrática, lineal o constante con respecto a la tensión de la siguiente manera:

$$
P_{Z I P}=P_{0}\left[p_{1} \bar{V}^{2}+p_{2} \bar{V}+p_{3}\right]
$$

y

$$
Q_{Z I P}=Q_{0}\left[q_{1} \bar{V}^{2}+q_{2} \bar{V}+q_{3}\right]
$$

donde $P_{0}$ y $Q_{0}$ son las condiciones iniciales, $p_{1}$ a $p_{3}$ y $q_{1}$ a $q_{3}$ son los parámetros que representan la proporción de cada componente. El modelo ZIP ha sido utilizado ampliamente como un modelo por defecto en las industrias de servicio publico (Hou et al., 2011).

La dependencia de la carga con la frecuencia es representada en el modelo exponencial como:

$$
P=P_{0}(\bar{V})^{a}\left(1+k_{p f} \Delta f\right)
$$

y

$$
Q=Q_{0}(\bar{V})^{a}\left(1+k_{q f} \Delta f\right) .
$$

A su vez es posible escribir la dependencia de la carga con la frecuencia utilizando el modelo ZIP de la siguiente forma:

$$
P=P_{Z I P}\left(1+k_{p f} \Delta f\right)
$$

y

$$
Q=Q_{Z I P}\left(1+k_{q f} \Delta f\right),
$$

donde $\Delta f$ es la desviación de frecuencia $\left(f-f_{0}\right)$. Típicamente, el rango de valores para las constantes $k_{p f}$ y $k_{q f}$ son:

$$
0 \leq k_{p f} \leq 3
$$




$$
-2 \leq k_{q f} \leq 0
$$

La frecuencia del bus usualmente no es una variable de estado en el modelo del sistema utilizado para realizar el análisis de estabilidad.

\subsubsection{Cargas dinámicas}

La respuesta de la mayoría de las cargas compuestas es rápida a variaciones de tensión y frecuencia y la respuesta de estado estacionario es alcanzada rápidamente. De esta manera la utilización de modelos estáticos está justificada en la mayoría de los casos. Sin embargo, existen situaciones donde resulta conveniente tener en cuenta el modelo dinámico de la carga. Los problemas que requieren un modelo dinámico de la carga son: estudios de oscilaciones interáreas, estabilidad de tensión, estudios de sistemas con una gran concentración de motores de inducción, etc.

Desde el colapso del sistema eléctrico de potencia en Suecia en el año 1983 se han empezado a utilizar modelos que emplean motores de inducción para el estudio de colapsos de tensión en un sistema. A partir de ensayos experimentales en los trabajos Hill (1993); Karlsson y Hill (1994) se presenta un modelo no lineal de una carga con recuperación exponencial que captura los comportamientos observados en buses de alta tensión, el cual incluye motores de inducción.

En los trabajos de Hill (1993) y Karlsson y Hill (1994) se asume un modelo dinámico de primer orden, que corresponde con el comportamiento del sistema. El modelo de la carga dinámica queda representado como:

$$
T_{p} \dot{P}_{d}+P_{d}=P_{s}(V)+k_{p}(V) \dot{V}
$$

donde

$$
P_{s}(V)=P_{0}\left(\frac{V}{V_{0}}\right)^{a}
$$

La solución para la ecuación diferencial (3.33) es:

$$
P_{d}(t)=P_{s}\left(V_{1}\right)+\left[P_{s}\left(V_{0}\right)-\frac{1}{T_{p}} K_{p}\left(V_{0}\right)-P_{s}\left(V_{1}\right)+\frac{1}{T_{p}} K_{p}\left(V_{1}\right)\right] e^{\frac{t-t_{0}}{T_{p}}}
$$

donde $t>t_{0}$. Para tener en cuenta otro tipo de comportamientos es posible construir un modelo de orden superior.

Otro modelo que incluye un motor de inducción y una carga PQ es debido a los trabajos de Walve (Walve, 1986). El motor de inducción es especificado a través de su demanda de potencia activa y reactiva en función de la tensión en el punto de conexión y de la frecuencia. El modelo combinado para el motor y la carga PQ es (Dobson y Chiang, 1989):

$$
\begin{gathered}
P_{d}=P_{0}+P_{1}+k_{p w} \dot{\delta}+k_{p v}(V+T \dot{V}), \\
Q_{d}=Q_{0}+Q_{1}+k_{q w} \dot{\delta}+k_{q v} V+k_{q v 2} V^{2}
\end{gathered}
$$


donde $P_{0}$ y $Q_{0}$ son las potencias constantes activa y reactiva del motor de inducción, $P_{1}$ y $Q_{1}$ representan la carga $\mathrm{PQ}, V$ es la tensión en el punto de conexión y $\dot{\delta}$ es la frecuencia. Los valores de las constantes $k_{p w}, k_{p v}, k_{q w}, k_{q v}$ y $k_{q v 2}$ para cargas típicas pueden consultarse en Dobson y Chiang (1989). Existen otros modelos para la carga que no dependen de la frecuencia, en particular en Hill (1993) se presenta un modelo simplificado a partir de Dobson y Chiang (1989).

\subsection{Generador sincrónico}

Los generadores sincrónicos trifásicos representan la principal fuente de energía eléctrica en sistemas de potencia. Los impulsores típicos para los generadores sincrónicos pueden ser motores diésel, turbinas de vapor, gas e hidráulicas, etc. Estos impulsores son los responsables de imponer el par mecánico en el eje del generador sincrónico.

La velocidad del rotor está dada por:

$$
n=\frac{120 f}{P_{f}},
$$

donde $n$ es la velocidad en rev/min, $f$ es la frecuencia en Hertz y $P_{f}$ es el número de polos en el rotor.

El comportamiento de un generador sincrónico puede modificarse a través del par mecánico impulsor $\left(T_{m}\right)$ y la tensión de campo $\left(E_{f d}\right)$. El dispositivo físico que controla el par aplicado al eje del generador se denomina impulsor y el dispositivo que controla $E_{f d}$ es llamado excitador. Si un generador sincrónico es utilizado en un sistema eléctrico de potencia, y este es utilizado en un rango amplio de funcionamiento, debe ser capaz de participar en el intento de mantener la tensión y la frecuencia constante en los bornes del generador. Esto se logra ajustando la tensión de campo y el par mecánico impulsor aplicado al generador sincrónico para diferentes puntos de equilibrio del sistema (Sauer y Pai, 1998).

Generalmente, un sistema de generación sincrónica que es impulsado por una turbina de vapor es controlado mediante el combustible del impulsor, el control de velocidad conocido como "governor" y la excitación. En la Figura 3.3 se puede observar un esquema de un generador sincrónico con sus sistemas de control.

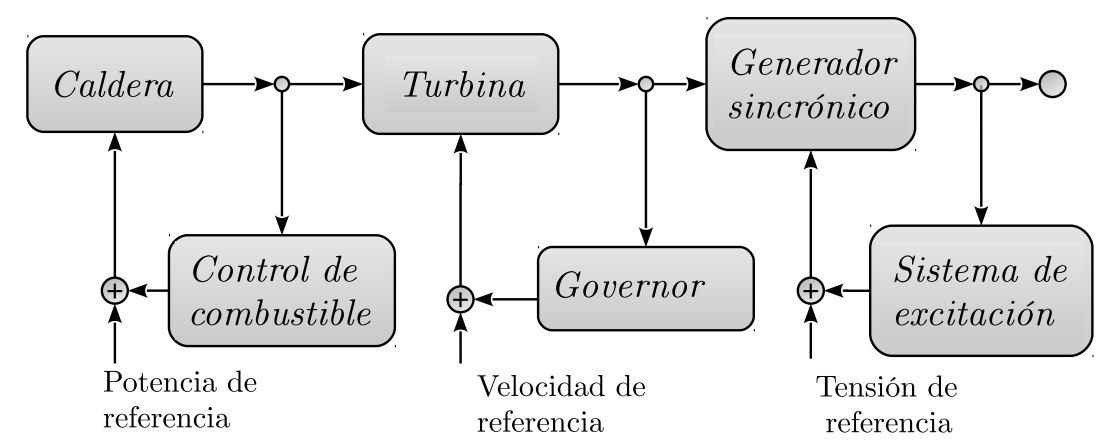

Figura 3.3: Sistema de Control para un sistema de generación sincrónica.

Para estudiar la estabilidad de un sistema de potencia se puede considerar que está operando con una carga en estado estacionario y que en un determinado momento el sistema 
es perturbado. Usualmente, estas perturbaciones causan un transitorio que es de naturaleza oscilatoria. Si el sistema de potencia es estable las oscilaciones son amortiguadas. Si no hay variaciones en el consumo de potencia por parte de la carga el sistema regresa al punto donde estaba operando y si existe una variación el sistema se mueve a un nuevo punto de trabajo.

La estabilidad en las máquinas sincrónicas y en las líneas de interconexión son problemas comunes en sistemas de potencia.

\section{Oscilaciones en líneas de interconexión}

En un sistema de potencia que opera de forma normal, perturbaciones que ocurren en la carga pueden producir variaciones de potencia de forma aleatoria. Debido a este efecto aleatorio cada generador está en continua oscilación con respecto a los otros sistemas de generación. Normalmente estas oscilaciones no son perjudiciales, aunque la situación es diferente cuando la línea de interconexión conecta dos grupos de generación de potencias elevadas. Esto representa un problema a tener en cuenta debido a que un pequeño ajuste en el control de uno de los sistemas de generación puede implicar una variación considerable de potencia en la línea de interconexión.

\section{Estabilidad en las máquinas sincrónicas}

En un sistema de potencia, un desbalance entre la generación y la carga puede iniciar un transitorio que causa que el rotor del generador sincrónico oscile. Esta oscilación es debida a que las fluctuaciones de potencia en la red provocan que el par en la máquina varíe. Si este par es lo suficientemente grande entonces el rotor de alguna de las máquinas puede oscilar y de esta forma se puede perder el sincronismo.

\section{Ecuaciones de swing de un generador sincrónico}

Durante un transitorio, producto de una perturbación en el sistema de potencia, visto desde un generador sincrónico las variables que se modifican son la tensión en el punto de conexión, el ángulo del rotor y la frecuencia. La señal que se utiliza para comprobar la estabilidad en un generador sincrónico es el ángulo del rotor medido con respecto a un marco de referencia rotante.

Las ecuaciones de relevancia en el análisis de estabilidad de un sistema son las expresiones que describen el desbalance entre el par electromagnético y el par mecánico de las máquinas individuales. Las ecuaciones de swing representan las características mecánicas de la máquina sincrónica en el estudio de la estabilidad.

En un generador el par impulsor es el par mecánico $T_{m}$ y el par de carga es el par electromagnético $T_{e}$, de esta forma, el par de aceleración está dado por:

$$
T_{a}=T_{m}-T_{e} .
$$

Luego, un valor positivo del par $T_{a}$ acelera la máquina, mientras que un valor negativo la 
frena. La expresión de movimiento es:

$$
J \frac{d \omega_{1}}{d t}=T_{a}=T_{m}-T_{e}
$$

donde $J$ es el momento de inercia combinada del generador y la turbina y $\omega_{1}$ es la velocidad angular del rotor.

Entonces, las expresiones de oscilación o de swing como dos ecuaciones diferenciales de primer orden son:

$$
\begin{gathered}
\dot{\delta_{1}}=\omega_{1}-\omega_{r}, \\
M_{1} \dot{\omega}_{1}+D_{1} \omega_{1}=P_{m}-P_{e},
\end{gathered}
$$

donde $\omega_{r}$ es la frecuencia de referencia del sistema, $M_{1}=J \omega_{1}$ es la constante de inercia, $D_{1}$ es la componente interna de fricción de la máquina, $P_{m}$ es la potencia mecánica suministrada por el impulsor y $P_{e}$ es la potencia eléctrica (inyectada a la red eléctrica). En el contexto de una red eléctrica pequeña y con el objetivo de aplicar técnicas de análisis y control para la misma es posible simplificar el modelo del generador sincrónico introduciendo el ángulo relativo del mismo, como se comenta en Sauer y Pai (1998). Esta característica es utilizada en el capítulo de aplicaciones para modelar el generador sincrónico.

Las ecuaciones de swing capturan la dinámica del generador sincrónico bajo la suposición de flujo constante en el bobinado del rotor, par mecánico constante y la ausencia de reguladores de tensión (Salam et al., 1984).

\section{Control de la velocidad y la frecuencia del generador sincrónico}

El impulsor del generador sincrónico suministra los mecanismos para controlar la velocidad del rotor del mismo y de esta forma la frecuencia de la tensión en los bornes de la máquina. Para controlar automáticamente la velocidad del rotor un dispositivo debe sensar la velocidad o la frecuencia. Con esta medición y el valor deseado de velocidad es posible generar una señal que ejerza una acción correctiva sobre la velocidad de la máquina. En este sentido, se desarrolla un modelo de un gobernador hidráulico para el sistema de la Figura 3.3 de forma genérica. Las ecuaciones del sistema describen la apertura de una válvula que controla el paso de vapor en un turbina, el cual está dado por:

$$
T_{s v} \frac{d P_{s v}}{d t}=-P_{s v}+P_{c}-\frac{1}{R_{d}}\left(\frac{\omega}{\omega_{s}}-1\right),
$$

donde $P_{s v}$ es la potencia, dada por la posición de la válvula, $P_{c}$ es la potencia en la carga, $\omega$ es la velocidad del eje, $\omega_{s}=2 \pi f_{s}$, con $f_{s}$ la frecuencia de la señal de tensión y por último una velocidad de regulación $R_{d}$ definida como:

$$
R_{d} \approx \frac{2 \pi D}{\omega_{s}}
$$

con una velocidad de decaimiento $D$ expresada en $\mathrm{Hz} /(\mathrm{PU}) \mathrm{MW}$. La apertura de la válvula de combustible tiene restricciones en los valores máximos y mínimos, dados por:

$$
0 \leq P_{s v} \leq P_{s v}^{\max }
$$


Además de los límites para la apertura de la válvula de combustible también resulta de interés restringir la velocidad de apertura de la misma. Este modelo es válido para pequeñas variaciones. Además, para que el sistema no presente error de estado estacionario es necesario añadir un control integral (Sauer y Pai, 1998).

\section{Control de tensión en generadores sincrónicos}

El sistema de excitación de campo proporciona los mecanismos para controlar la magnitud de la tensión en los terminales del generador sincrónico. En los generadores de gran tamaño el sistema de excitación de campo es una parte importante del diseño global, debido a que este debe estabilizar la tensión en los bornes ante una variación en la carga.

El sistema de control de excitación consiste de un excitador y un AVR (Automatic voltage regulator). Este último regula la tensión generada controlando la cantidad de corriente inyectada al bobinado de campo por el excitador. Un modelo simple para el control de la tensión es presentado en (Sauer y Pai, 1998):

$$
T_{A} \frac{d V_{r}}{d t}=-V_{R}+K_{a} V_{i n},
$$

donde $V_{R}$ es la entrada para el excitador, $V_{i n}$ es la diferencia de tensión $\left(V_{\text {ref }}-V_{m}\right), V_{m}$, es la tensión medida en los bornes del generador sincrónico, $T_{A}$ es una constante de tiempo y $K_{a}$ es una ganancia. El actuador del sistema de excitación esta acotado por:

$$
V_{R}^{\min } \leq V_{R} \leq V_{R}^{\max } .
$$

\subsection{Sistemas de almacenamiento de energía}

Cuando ocurre una perturbación en la red eléctrica producto de una variación rápida en la carga, los generadores sincrónicos no siempre pueden actuar con la suficiente rapidez para mantener la estabilidad del sistema. Una solución para este problema la puede aportar un sistema de almacenamiento de energía. Con el desarrollo de la electrónica de potencia los sistemas de almacenamiento de energía pueden ser controlados para entregar o absorber potencia activa o reactiva de forma flexible (Tang et al., 2014). De esta forma, las tecnologías de almacenamiento de energía (ESS, por la sigla en inglés de Energy Storage System) pueden proporcionar valiosos beneficios adicionales para mejorar la calidad de la energía y la fiabilidad del suministro de energía eléctrica.

En un sistema de generación distribuida la incorporación de un sistema de almacenamiento de energía conlleva un aumento de la calidad de la energía cuando el sistema esta operando de forma autónoma (Tang et al., 2014). Como una consecuencia de la utilización de ESS, se reduce el consumo de combustibles fósiles asociados a sistemas de generación distribuida. Además, una motivación adicional se ha producido con la aplicación de ESS a micro redes y a redes inteligentes (Boicea, 2014).

Diferentes tecnologías fueron estudiadas para poder implementar sistemas de almacenamiento de energía para sistemas de potencia en Ribeiro et al. (2001), las cuales resumiremos a continuación. 


\subsubsection{Tecnologías en sistemas de almacenamiento}

Los dispositivos que pueden almacenar energía eléctrica sin convertirla a otro tipo de energía se denominan ESS directos. En esta categoría están los dispositivos que utilizan el campo electromagnético para almacenar energía y que están construidos empleando capacitores e inductores. En la Figura 3.4 se puede observar dos esquemas típicos de sistemas de almacenamiento de energía directo, basadas en supercapacitores y superconducción conectados a convertidores de potencia.

Los dispositivos que convierten la energía en otro tipo de energía se denominan ESS indirectos. La transformaciones pueden ser a energía mecánica o química. En la Figura 3.5 se puede observar un diagrama con un sistema de almacenamiento de energía indirecto, donde se muestran las diferentes tecnologías asociadas. El dispositivo que almacena en forma de energía cinética es el volante de inercia o Flywheel, mientras que los elementos que almacenan energía en forma de energía potencial son los sistemas que utilizan aire comprimido (CAES, por sus siglas en inglés Compressed Air Energy Storage) y sistemas de bombeo de agua (HPES, por sus siglas en inglés Hydro Pumped Energy Storage), los dispositivos que se utilizan para almacenar energía de forma química están basados en baterías (Grbovic, 2014; Boicea, 2014).

Dos factores caracterizan a los sistemas de almacenamiento de energía. Uno de ellos es la cantidad de energía que puede ser almacenada en el dispositivo. La otra es la tasa con la cual el ESS puede absorber o entregar energía (Ribeiro et al., 2001).

En la Figura 3.6 se puede observar una comparación entre diferentes tecnologías empleadas en sistemas de almacenamiento de energía. La potencia que puede suministrar el sistema se representa en el eje de las abscisas, mientras que en el eje de las ordenadas se puede observar el tiempo en el cual el ESS puede entregar energía. También se señala la aplicación del ESS, es decir, si se puede aplicar a grandes sistemas de energía, a líneas de transmisión y distribución de energía o si se emplea para mejorar la calidad de potencia (Boicea, 2014).

\section{Sistemas de almacenamiento basados en baterías (BSS)}

Los sistemas de almacenamiento de energía basados en baterías fueron los pioneros, aun hoy siguen siendo la tecnología más accesible en el mercado. La carga de la batería está basada en una reacción interna generada por una tensión aplicada a los bornes de la misma. Este proceso es reversible. Actualmente, los BSS se utilizan para mejorar el desempeño de los sistemas de potencia como así también para sistemas de transmisión y distribución, como se observa en la Figura 3.6.

\section{Sistemas de almacenamiento de energía magnética por superconducción (SMES)}

Un SMES, (por su sigla en inglés de Superconducting Magnetic Energy Storage), es un dispositivo que tiene la capacidad de almacenar energía en el campo magnético generado por una corriente continua que circula en una bobina superconductora, la cual está refrigerada.

La energía almacenada en un SMES es:

$$
E=\frac{1}{2} L I^{2}
$$




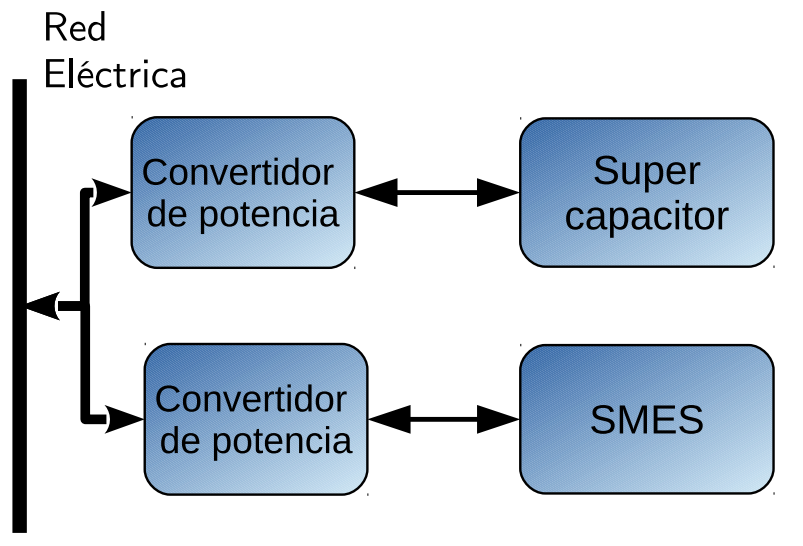

Figura 3.4: Esquema de un ESS directo.

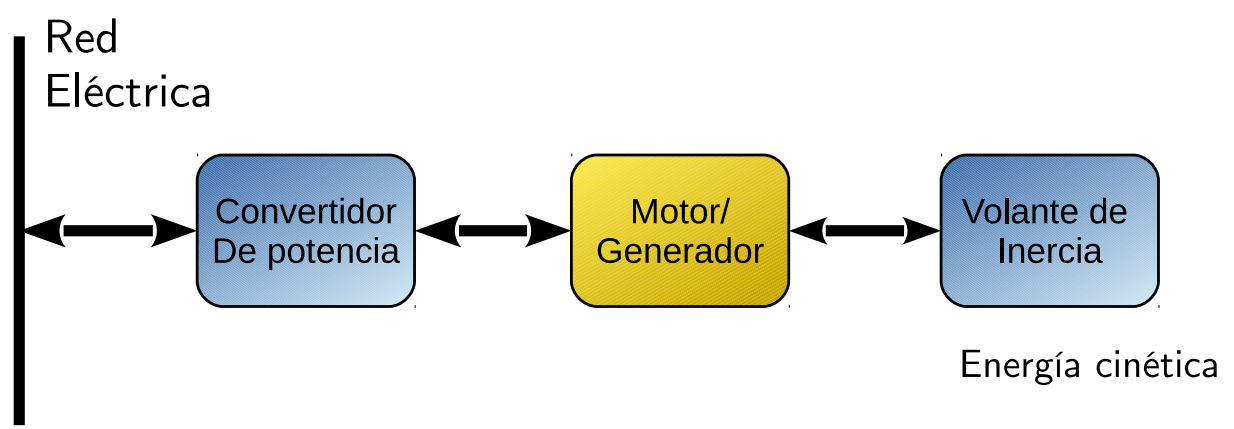

Figura 3.5: Sistemas de almacenamiento indirecto.

donde $L$ es la inductancia de la bobina, $I$ es la corriente continua que circula en la misma. Empleando SMES se logran corrientes de cientos de Amperes.

Los sistemas de almacenamiento basado en SMES tienen una buena eficiencia y pueden responder rápidamente, estas características hacen que el sistema se pueda aplicar en diferentes niveles de potencia. Actualmente, los sistemas de almacenamiento basados en SMES pueden ser utilizados en sistemas tanto de distribución de energía como de transmisión, como se observa en la Figura 3.6, pudiendo entregar potencias en el orden de los cientos de MW por un tiempo del orden de los segundos (Ribeiro et al., 2001).

\section{Sistemas de almacenamiento de energía basados en un volante de inercia}

Los sistemas de almacenamiento de energía FES, (por su sigla en inglés Flywheel Energy Storage) están compuestos por un volante de inercia acoplado a una máquina eléctrica impulsora y un convertidor de potencia que permite operar el sistema impulsor en un rango amplio de funcionamiento (Figura 3.5). La energía almacenada depende del momento de inercia $(I)$ y de la velocidad de rotación $\omega$ de la siguiente forma:

$$
E=\frac{1}{2} I \omega^{2} .
$$

Para maximizar la energía almacenada podemos variar la inercia o la velocidad de giro del volante o ambas. Una opción es incrementar la inercia utilizando una masa de acero con 


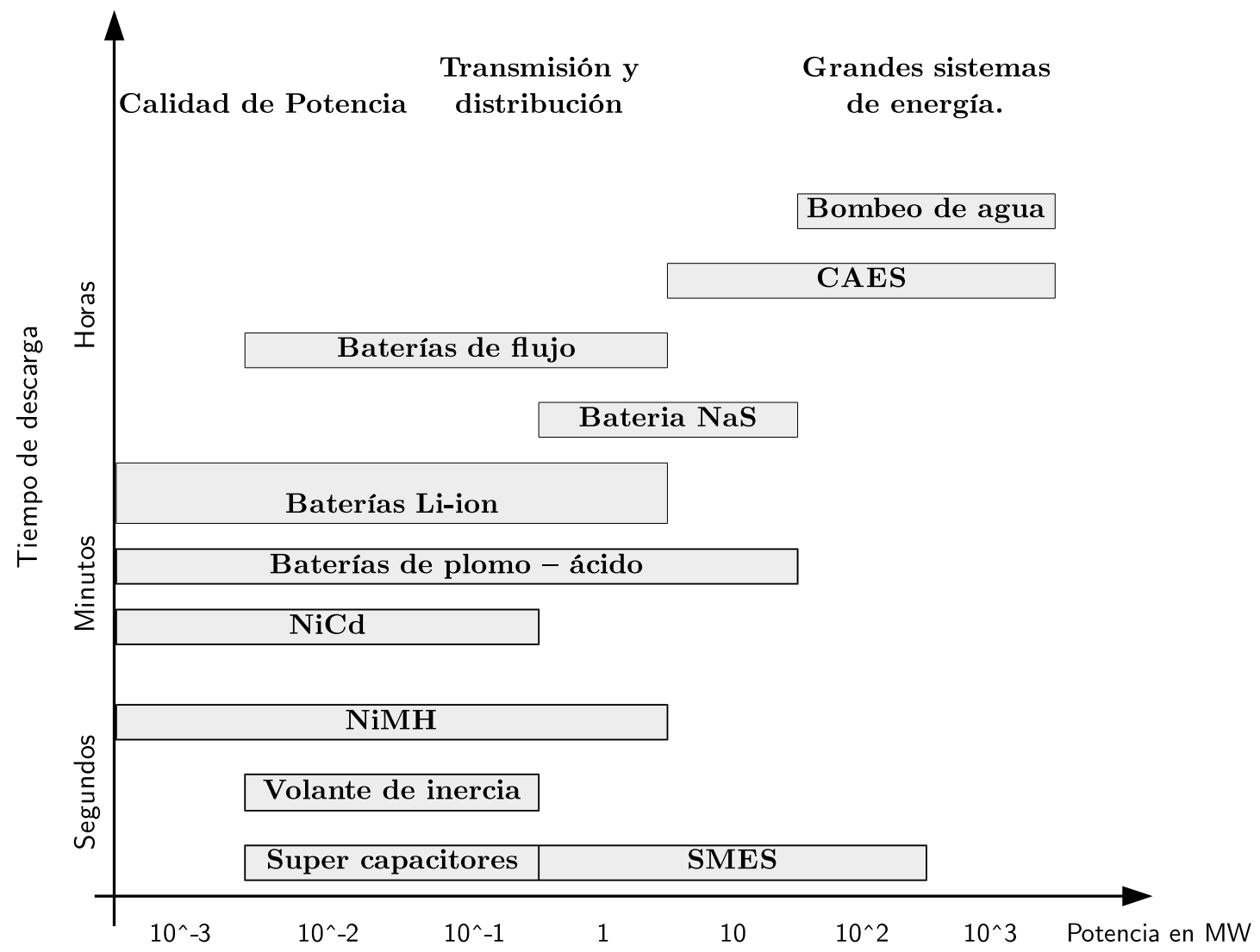

Figura 3.6: ESS, aplicados a un sistema de potencia.

un gran radio con una velocidad de aproximadamente de 10000 revoluciones por minuto. La segunda estrategia de diseño es producir volantes con un rotor liviano a una velocidad de rotación mayor (por ejemplo de $100.000 \mathrm{rpm}$ ).

\section{Supercapacitores}

La energía almacenada en un capacitor es:

$$
E=\frac{1}{2} C V^{2}
$$

donde $C$ es la capacitancia del condensador y $V$ es la tensión entre los bornes del capacitor. El sistema de almacenamiento basado en un super o ultra capacitor se implementa con un convertidor, como se muestra en la Figura 3.4.

El super o ultra capacitor es un capacitor electroquímico, el cual está compuesto de dos electrodos conductores porosos y un separador que impide el contacto directo entre ellos. El super capacitor es un capacitor no lineal, donde la capacitancia es función de la tensión y está dada por:

$$
C(V)=C_{0}+k_{c} U_{0}
$$

donde $C_{0}$ representa la capacitancia electrostática del capacitor, $U_{0}$ es la tensión en los bornes y $k_{c}$ es el coeficiente que representa la capa difusa del super capacitor (Grbovic, 2014). 


\section{Sistemas de almacenamiento de energía por bombeo de agua}

Esta tecnología representa la solución más eficiente para almacenar grandes cantidades de energía. Hay momentos en los cuales la demanda de energía eléctrica puede incrementarse o caer. De esta manera entonces se puede aprovechar cuando la demanda cae para bombear agua desde un acuífero a una altura inferior a uno de altura superior (Boicea, 2014). Cuando es necesario utilizar esta energía almacenada la bomba trabaja como una turbina e impulsa el generador. De esta forma el generador impulsado por la turbina inyecta energía a la red eléctrica a través de un convertidor.

La energía almacenada en el depósito superior está dada por la energía potencial cuya expresión es:

$$
E=V_{0} \rho g H,
$$

donde $V_{0}$ es el volumen del depósito superior, $\rho$ es la densidad del agua, $g$ es la gravedad y $H$ es la diferencia de altura entre la bomba y el depósito de agua superior. Los sistemas de almacenamiento basados en el bombeo de agua son utilizados en sistemas de gran escala y alta potencia como se indica en la Figura 3.6.

En nuestro país existen centrales de bombeo de agua en la provincia de Córdoba y Mendoza. La central ubicada en Córdoba tiene una potencia instalada de $750 \mathrm{MW}$ (Trombotto, 2004).

\subsubsection{Sistemas de almacenamiento de energía aplicados a sistemas de generación de energía eólica}

Si la conexión a la red eléctrica es débil y existen problemas para mantener la calidad de la energía se pueden instalar sistemas de almacenamiento de energía para tratar de mitigar los problemas de la conexión de aerogeneradores en redes débiles, en particular para mantener la estabilidad de la potencia y el control de la tensión en la red. La adición de un ESS puede ayudar a incrementar la penetración de energías renovables (Hamsic et al., 2007).

Generalmente, los sistemas de generación autónomos, es decir que no están conectados a una red de distribución, emplean un generador sincrónico para suministrar energía a las cargas eléctricas conectadas al mismo. Si existe la posibilidad de instalar aerogeneradores, la carga en el generador sincrónico se reduce. De esta forma el consumo de combustible y el costo de la energía se reduce por el aporte de energía eólica. El generador sincrónico puede operar en un mínimo de carga, típicamente del orden del $40 \%$ de la potencia nominal (Kaldellis, 2010). La operación por debajo del mínimo de potencia del sistema de generación sincrónica aumenta el mantenimiento y reduce la vida útil del motor diésel. Existen varias formas de reducir este inconveniente. Por ejemplo la operación del generador sincrónico puede ser programada de acuerdo a la potencia eólica disponible y la demanda. Algunos sistemas pueden aprovechar el excedente de energía para producir algún otro tipo de producto. También se puede emplear el remanente de energía para calentar agua, producir hidrógeno o para desalinizar agua, etc.

Como parte de este trabajo de tesis se consideraron los siguientes sistemas:

En Toccaceli et al. (2012a) se propuso la topología y la estrategia de operación de un sistema de generación distribuida renovable que alimenta una planta de ósmosis inversa para la producción de agua dulce en zonas costeras. La planta desalinizadora está conectada a una red 
débil que le proporciona asistencia y es alimentada a través de un aerogenerador de velocidad variable equipado con un generador BDFIG (por su siglas en inglés Brushless Double Fed Induction Generator) donde el estator principal de esta máquina está directamente conectado al sistema eléctrico de potencia, mientras que el bobinado de control del estator es controlado por un convertidor electrónico. Además, se empleó un sistema de almacenamiento de energía basado en un volante de inercia para atenuar las perturbaciones debidas a variaciones en el recurso eólico.

En Toccaceli et al. (2012b) se presentó la topología y las estrategias de control para un sistema de generación distribuida con capacidad de operar en isla. En este trabajo se consideró una pequeña micro red construida con un aerogenerador equipado con un generador asincrónico de rotor bobinado (DFIG), una carga, un sistema de almacenamiento basado en super capacitores y un generador sincrónico impulsado por un motor diésel/gas. En esta arquitectura, el sistema de almacenamiento de energía basado en super capacitores se conecta a la red a través de un inversor trifásico de tensión. En operación en isla se prioriza la reducción del consumo de combustible por parte del motor diésel. Esta capacidad de operación, hace al sistema particularmente útil en áreas con frecuentes interrupciones en el suministro eléctrico.

Tanto en Toccaceli et al. (2012a) como en Toccaceli et al. (2012b) se utilizaron los conceptos relacionados al espectro típico de distribución de la energía del viento desarrollado por Van der Hoven. Utilizando este espectro se sintonizó un filtro con el cual se realiza el control del sistema de almacenamiento de energía.

\subsection{Micro red}

Aspectos económicos, tecnológicos y ambientales están cambiando la perspectiva de la generación y del transporte de la energía eléctrica. En este sentido iniciativas relacionadas al cambio climático, las cuales involucran reducción de la emisión de $\mathrm{CO}_{2}$, reducción de contaminación del medio ambiente e incentivos en la utilización de energías renovables impulsan el desarrollo de nuevas tecnologías.

Los sistemas de generación distribuida (DER, por su sigla en inglés Distributed Energy Resources) están compuestos por una amplia gama de sistemas generación de energía eléctrica como sistemas fotovoltaicos, turbinas a gas, microturbinas y turbinas eólicas que impulsan generadores de inducción, etc. Los sistemas de generación distribuida están emergiendo como un nuevo paradigma con respecto a la generación de energía eléctrica tradicional. Estas nuevas tecnologías tienen como premisa la calidad en el servicio eléctrico.

Utilizando generación distribuida en sistemas de distribución se reduce la distancia entre los sistemas de generación y las cargas. Acercar los sistemas de generación a las cargas potencialmente puede contribuir con el perfil de tensión, a reducir el cuello de botella en la distribución y transmisión de energía y además, posponer la inversión de nuevos sistemas de generación y transmisión en gran escala. Los conceptos de micro red con sus controladores locales y su asistencia en la calidad de la energía permiten la integración escalable de generación de energía local y cargas dentro de una red eléctrica existente permitiendo una alta penetración de generación distribuida renovable (Bidram et al., 2014). 


\subsubsection{Conceptos de Micro red}

El departamento de energía de los Estados Unidos (DOE) definió el concepto de micro red como un conjunto de cargas interconectadas y recursos de energía distribuidos con un claro objetivo de actuar como una sola unidad controlable con respecto a la red (Parhizi et al., 2015). Las micro redes pueden ser híbridas de corriente alterna (AC) y corriente continua (DC) y además pueden operar conectadas a una red de distribución o pueden operar de forma aislada.

Existen muchas otras definiciones de micro redes pero todas involucran un grupo de cargas, sistemas de generación y sistemas de almacenamiento de energía conectados entre sí (Montoya et al., 2013).

Los objetivos básicos de una micro red son mejorar la confiabilidad, promover la alta penetración de fuentes renovables, aislamiento dinámico y la mejora de la eficiencia a través del aprovechamiento del calor residual a través de sistemas de generación utilizando ciclos combinados. Los conceptos relacionados con una micro red tienen en cuenta la alta penetración de generación distribuida sin requerir el rediseño del sistema de distribución (Piagi y Lasseter, 2006).

La implementación de sistemas de generación distribuida requiere controlar un número significativo de unidades de generación, lo cual ha sido un disparador para el desarrollo de sensores rápidos y el desarrollo de nuevas estrategias de control.

En términos de seguridad de la fuente de energía, múltiples unidades de generación son más eficientes que un solo sistema de generación de energía. Es decir, estas pequeñas unidades de generación pueden hacer un mejor seguimiento de una carga que un gran generador sincrónico. Además, teniendo múltiples sistemas de generación en una red eléctrica si una de las unidades falla el resto debería entregar más potencia para que el sistema continúe operando sin inconvenientes. De esta forma el sistema eléctrico de potencia se vuelve mucho más confiable ya que no depende de un único sistema de generación de energía eléctrica.

Los sistemas de comunicación en una micro red son un medio para intercambiar información y controlar diversos elementos de la micro red con el objetivo de llevar a cabo una correcta operación y protección de la misma.

\subsubsection{Configuración típica de una micro red}

Con el objetivo de introducir los componentes de una micro red se describe el esquema de una micro red típica mostrada en la Figura 3.7. Dicha micro red, que es presentada en Chowdhury et al. (2009), consiste de cargas y micro fuentes conectadas a través de una red de baja tensión en una red de distribución. La micro red consiste de tres alimentadores A, B y C que proveen energía a las cargas eléctricas. En el esquema hay dos micro fuentes de ciclo combinado (CHP, por su sigla en inglés Combined Heat and Power) y dos micro fuentes no CHP (NCHP) y sistemas de almacenamiento de energía. Las micro fuentes y los ESS se conectan a los puntos de conexión A y C.

Los elementos de generación deberían tener características del tipo plug and play, es decir capacidad para conectarse y desconectarse sin que se requiera una configuración especial en sus respectivos controladores locales. Estas fuentes están provistas de interfaces de potencia 


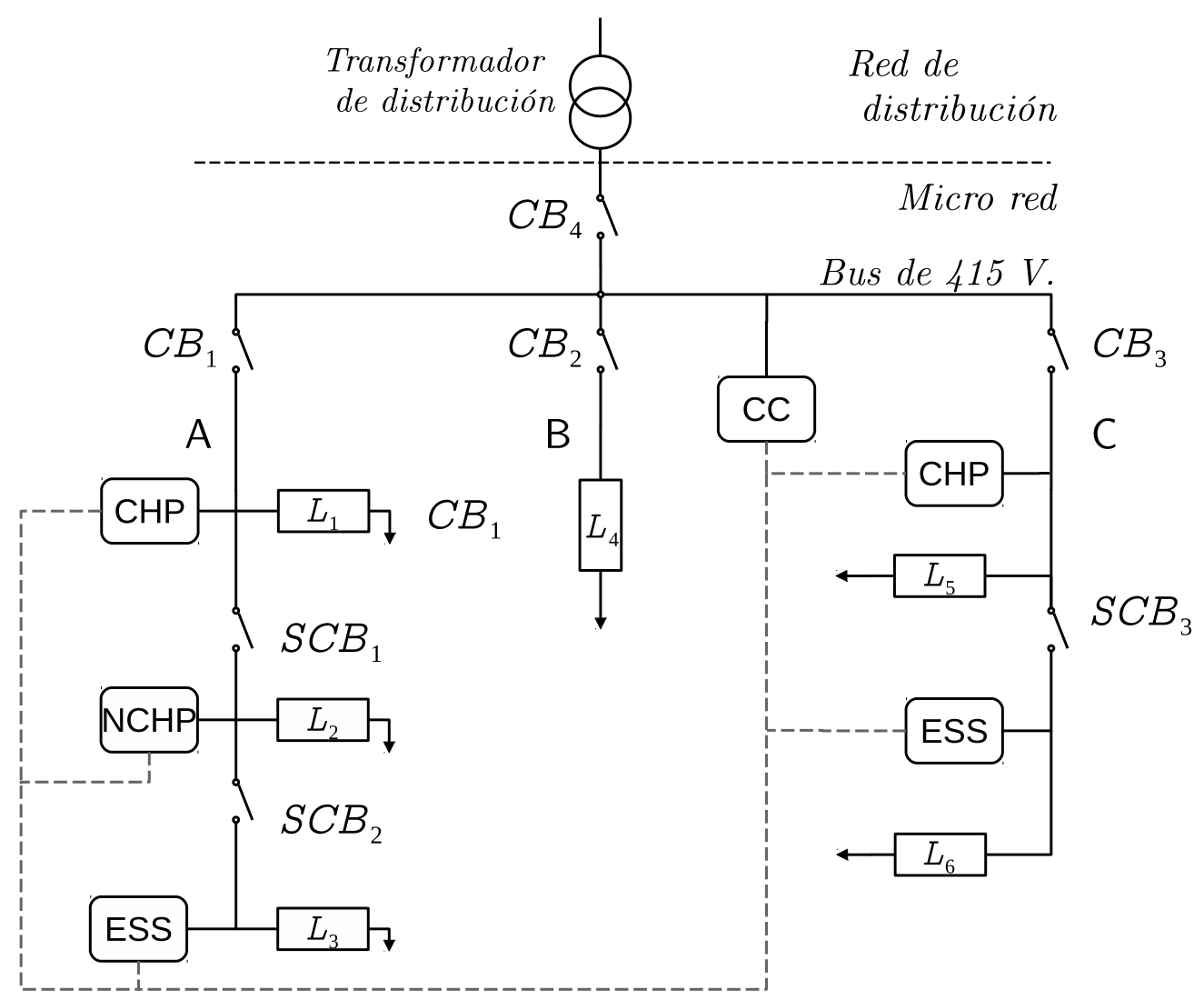

Figura 3.7: Diagrama de una micro red.

electrónicas para implementar el monitoreo y las funciones de protección durante la operación en isla y en red. En la micro red de la Figura 3.7 se definen cargas prioritarias que requieren un sistema ininterrumpido de aprovisionamiento de energía, las cuales se encuentran conectadas a los alimentadores A y C. Con el objetivo de simplificar el diagrama, todas las cargas no prioritarias que se encuentran conectadas al alimentador B se representan como $L_{4}$. La micro red es acoplada con la red de media tensión a través del punto común de conexión (PCC). El elemento que puede conectar y desconectar la micro red completa de la red principal es el interruptor $\left(C B_{4}\right)$. Además, los alimentadores $\mathrm{A}, \mathrm{B}$ y $\mathrm{C}$ pueden desconectarse utilizando los interruptores $C B_{1}, C B_{2}$ y $C B_{3}$. Las micro fuentes sobre el alimentador $\mathrm{A}$ o $\mathrm{C}$ pueden mejorar el perfil de tensión. Cada micro fuente y cada sistema de almacenamiento está gobernado por un sistema de control local.

En el modo conectado a la red la microgrid puede exportar o importar potencia con la red de distribución. En el caso que exista una perturbación en la red de distribución la micro red puede desconectarse y de esta forma la micro red trabaja como un sistema autónomo. La otra opción es abrir $C B_{1}$ y $C B_{3}$. Esta configuración permite suministrar potencia a las cargas $L_{1}, L_{2}, L_{3}, L_{5}$ y $L_{6}$ a través de los generadores conectados al bus A y C. Mientras que la carga $L_{4}$, la cual no es prioritaria será alimentada por la red de distribución. 


\subsubsection{Sistemas de control en una micro red}

Debido a la integración de sistemas de generación de energía eléctrica (renovables y no renovables) y de diferentes sub sistemas es un gran desafío el estudio de la topología y las estrategias aplicables a una micro red. En este sentido, las capacidades de los controladores de una micro red son cruciales para cumplir con los requerimientos de la misma, siendo el principal objetivo garantizar la calidad del suministro de potencia a la carga a pesar de los cambios en el sistema. En particular, son necesarios algoritmos de detección de aislamiento para asegurar una transición suave entre los modos de operación "conectado a la red" y "aislada" de la misma. Por otra parte, también los problemas debidos a diferentes fallas en la red deben ser tenidos en cuenta y el sistema de control de la micro red debe asegurar los valores de tensión y frecuencia apropiados (Guerrero et al., 2011) (Ustun et al., 2011).

Los controladores locales (CL) gestionan los sistemas de generación de energía eléctrica y las cargas controlables. Las principales funciones del controlador local de la micro fuente son administrar el flujo de potencia de la fuente y regular el perfil de tensión en el punto de conexión, donde se encuentra conectada la unidad de generación. Este controlador actúa de forma independiente, es decir, estas dos funciones deben ser llevadas a cabo sin la necesidad de comunicación con un control central.

El aspecto más significativo acerca de los controladores locales es que estos pueden monitorear la tensión y la corriente en el punto de conexión y con estas mediciones controlar la unidad de generación.

Existen varios tipos de estructuras de control, centralizado y descentralizado, para el adecuado funcionamiento de una micro red.

\section{Control centralizado de la micro red}

Mediante un control centralizado el intercambio de potencia entre los sistemas de generación y la carga pueden ser optimizados maximizando la producción local en función de los costos internacionales y las restricciones de seguridad (Chica Leal et al., 2015). Típicamente, en un sistema centralizado la operación y las estrategias de la micro red en diferentes modos de operación es controlado y coordinado por el controlador central, el cual toma decisiones periódicamente y envía los valores de referencia a los controladores locales (Katiraei et al., 2008).

El controlador central lleva a cabo la gestión y la protección de la micro red en su conjunto a través de los controles locales. Sus objetivos son:

- Mantener la tensión y la frecuencia en la carga a través de la inyección de potencia activa y de potencia reactiva a través de las unidades de generación.

- Establecer estrategias de optimización de la energía en la micro red. En este sentido el controlador central provee los valores de referencia para cada control local.

Cuando la micro red opera conectada a la red, las señales de control del módulo de manejo de energía están restringidas a las potencias activa y reactiva. El control local de tensión y del factor de potencia son ejercidos por el control local de las unidades de generación. De 
esta manera, la unidad de generación se puede ver como una carga controlable que trabaja con un factor de potencia unitario.

Cuando la micro red está trabajando de forma aislada de la red, la función principal del módulo es contribuir con los valores de referencia de la potencia activa y la tensión para los microcontroladores de las unidades de generación.

En un esquema centralizado la comunicación es necesaria para realizar el control de los dispositivos de generación (Parhizi et al., 2015). La comunicación entre el control central y las unidades de generación puede ser llevado a cabo mediante una red digital de servicios integrados (ISDN, por sus siglas en inglés Integrated Services for Digital Network) o mediante una línea de abonado digital asimétrica (ADSL, por sus siglas en inglés Asymmetric Digital Subscriber Line) provistas por un proveedor local de internet (ISP), etc (Hossain et al., 2014). También se puede emplear sistemas de comunicación por la misma red eléctrica, como PLC (por sus siglas en inglés Power Line Communications), o sistemas inalámbricos (Galli et al., 2011; Khan y Khan, 2012).

\section{Control de la micro red de forma descentralizada}

En los sistemas descentralizados, las unidades de generación comparten la carga de acuerdo a sus características y capacidades de control de la potencia. En este sentido, las unidades de control pueden utilizar una estrategia de control basada en las curvas de decaimiento, sin la necesidad de comunicación (Chica Leal et al., 2015).

Sin embargo, teniendo en cuenta que si el sistema debiera incluir funciones económicas, factores ambientales y requerimientos técnicos es necesario un sistema de comunicación para cumplir con estos objetivos. De esta manera, el controlador local se puede comunicar con cada elemento de la red para formar una gran entidad inteligente (Katiraei et al., 2008; Parhizi et al., 2015).

\section{Estrategias para la micro red}

Cuando la micro red se encuentra operando conectada a la red, es decir cuando se opera en modo seguimiento, los valores de tensión y frecuencia son impuestos por la red. Si se emplea el método no interactivo los sistemas de generación distribuida son operados de forma tal de obtener la máxima potencia disponible. Esto se puede lograr a través de seguimiento del punto de máxima potencia (MPPT) que garantizan la máxima extracción de potencia o una potencia constante. Estos métodos son aplicables a sistemas de generación eólica y solar (Ustun et al., 2011).

Cuando se emplea el método interactivo la potencia inyectada por los sistemas de generación distribuida depende de la carga y de la red. De esta forma, se puede lograr que toda la potencia que consume la carga sea provista por los sistemas de generación distribuida renovable economizando la energía que proviene de la red de distribución.

Cuando la micro red se encuentra operando en forma autónoma, se pueden emplear dos modos de operación. El método no interactivo implica mantener la tensión y la frecuencia constante. Esto puede ser logrado por algunas unidades de generación entonces el resto puede 
operar aportando potencia para su correcto balance. El segundo método emplea las curvas de decaimiento con el objetivo de compartir la carga por los sistemas de generación (Katiraei et al., 2008). Las curvas de decaimiento para una micro red son abordadas en la siguiente subsección.

\subsubsection{Curvas de decaimiento}

En la Figura 3.8 se muestra una unidad de generación de energía eléctrica en una micro red la cual está compuesta por una micro fuente, un convertidor electrónico y una línea de transmisión "L".

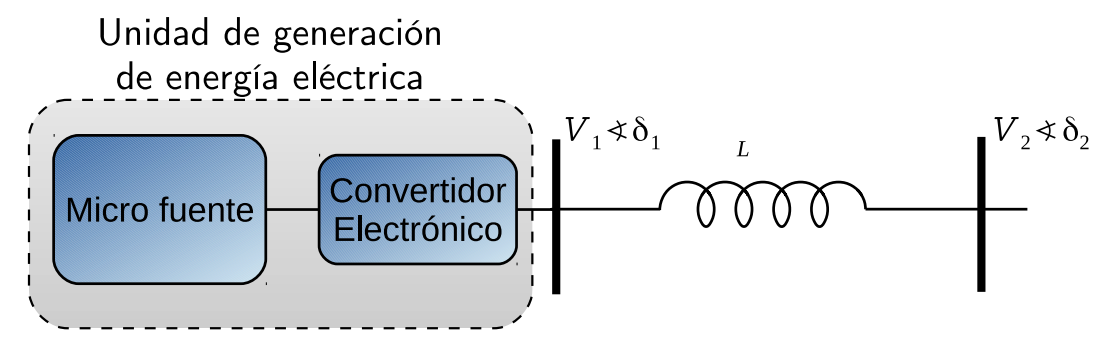

Figura 3.8: Esquema básico de un sistema de generación de energía.

El convertidor electrónico asociado a la micro fuente puede controlar la tensión $\left(V_{1}\right)$ y el ángulo de fase $\left(\delta_{1}\right)$. Generalmente la potencia activa en una micro red es controlada modificando la diferencia $\left(\delta_{1}-\delta_{2}\right)$ controlando el valor $\delta_{1}$, según la ecuación (3.4). La inyección de potencia reactiva es dada por la expresión (3.5), variando el módulo de la tensión $V_{1}$. Además de controlar la inyección de potencia activa y reactiva en una micro red es de utilidad controlar la tensión del punto de conexión de la unidad de generación o del sistema de almacenamiento. La tensión en el punto de conexión puede ser controlada utilizando una curva de decaimiento de tensión en función de la potencia reactiva $(\mathrm{Q}-\mathrm{V})$ como se muestra en la Figura 3.9.

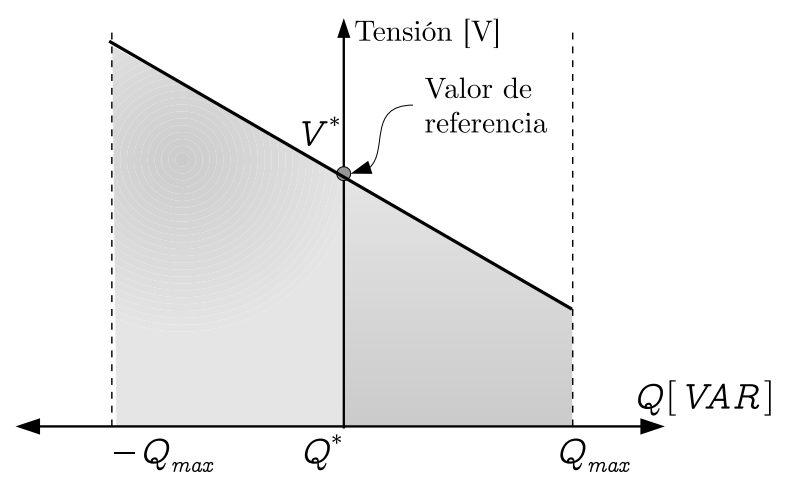

Figura 3.9: Curva decaimiento de Q-V.

La función que describe la curva de decaimiento está dada por (Guerrero et al., 2011):

$$
V=V^{*}-G_{q}(s)\left(Q-Q^{*}\right),
$$

donde $V^{*}$ es la tensión de referencia, $G_{q}(s)$ es una función de transferencia, $Q$ y $Q^{*}$ son las potencias reactiva medida y nominal, respectivamente. Generalmente, el valor de $G_{q}(s)$ es 
igual a $n$ y puede ser diseñado como: $n=\frac{\Delta V}{2 Q_{\max }}, \Delta V$ y $Q_{\max }$ es la máxima variación de tensión y la máxima potencia reactiva entregada por el inversor respectivamente.

La función del controlador es modificar la referencia de la potencia reactiva cuando la tensión es diferente a la tensión de referencia empleando la expresión (3.51), teniendo en cuenta que la potencia reactiva no tiene que superar el siguiente límite:

$$
Q_{\max }=\sqrt{\left(S^{2}-P^{2}\right)}
$$

\section{Curvas de decaimiento P-f}

La expresión para la curva de decaimiento para la potencia activa de una unidad de generación está dada por (Guerrero et al., 2011):

$$
\omega=\omega^{*}-G_{p}(s)\left(P-P^{*}\right),
$$

donde $\omega^{*}$ es el valor de referencia para la frecuencia, $G_{p}(s)$ es una función de transferencia, $P^{*}$ es la referencia para la potencia activa y $P$ es la potencia activa medida.

En la Figura 3.10 se observa la curva típica de decaimiento de la frecuencia en función de la potencia activa para una sola máquina. El bloque de control que implementa la curva de decaimiento es esquematizado en la Figura 3.11. Además, en la figura se representa el ángulo $\delta_{v}$ con el cual se ataca el inversor con el objetivo de controlar la potencia activa que este inyecta a la red (Lasseter y Paolo, 2006). Al bloque que implementa la curva de decaimiento

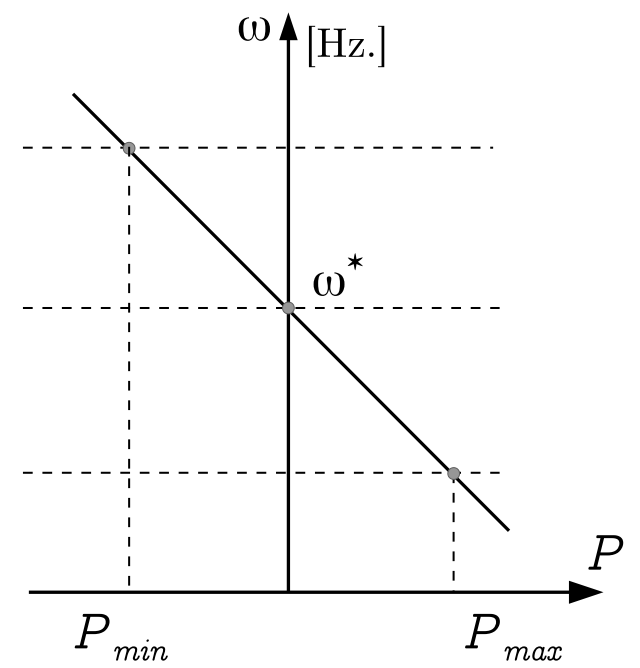

Figura 3.10: Curva de decaimiento de la potencia activa en función de la frecuencia para una máquina.

P-f ingresan tres señales las potencia medida $(P)$, la potencia deseada $\left(P^{*}\right)$ y la frecuencia nominal del sistema $\left(\omega^{*}\right), G_{p}(s)$ representa una función de transferencia, típicamente es el valor de la pendiente de la curva de decaimiento. $G_{p}(s)$ depende de la potencia máxima y del apartamiento de $\omega$ con respecto a la frecuencia nominal. Es decir:

$$
G_{p}(s)=\frac{\Delta \omega}{P_{\max }},
$$


donde $\Delta \omega$ es la máxima diferencia de frecuencia permitida y $P_{\max }$ es la máxima potencia entregada por el inversor.

El valor del ángulo $\delta_{v}$ es obtenido integrando en el tiempo el valor de $\omega$ desde $-\pi$ y $\pi$ (Lasseter y Paolo, 2006).

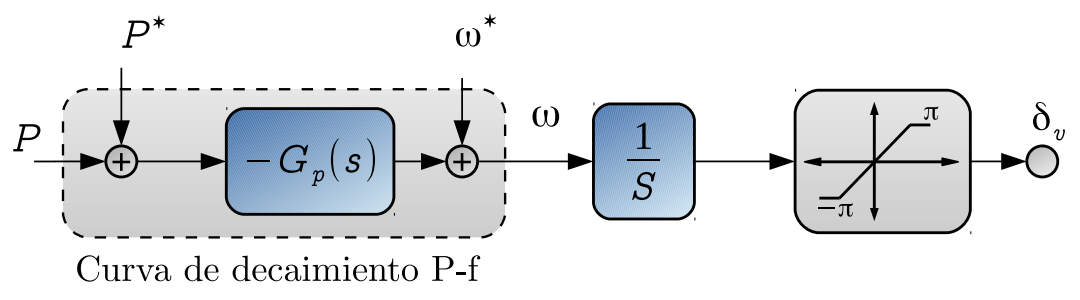

Figura 3.11: Cálculo del ángulo $\left(\delta_{v}\right)$ a partir de la característica de decaimiento.

\section{Curva de decaimiento para dos máquinas}

Los controladores de la micro red aseguran un cambio suave y automático en la transición del modo conectado a la red al modo aislado y viceversa. Para esto se pueden utilizar las curvas de decaimiento como método para lograr que las unidades de generación aporten potencia según sus capacidades. En la Figura 3.12 se muestran las curvas típicas de decaimiento P-f para dos unidades de generación conectadas al mismo bus.

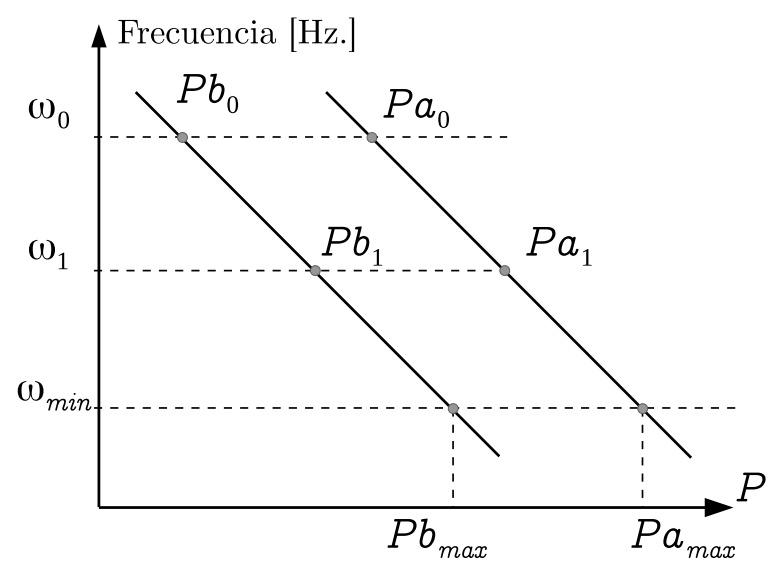

Figura 3.12: Curvas de decaimiento de la potencia activa en función de la frecuencia.

Los dos casos que son analizados es cuando la micro red está funcionando de forma aislada y cuando está conectada a la red de distribución ante una variación del consumo de la carga. Cuando la micro red está operando conectada a la red, las potencias que entregan las micro fuentes son $P a_{0}+P b_{0}$ y el resto de potencia la suministra la red de distribución. Como se mencionó previamente la red de distribución impone la frecuencia en el bus y las dos unidades de generación siguen esta frecuencia operando a la frecuencia $\omega_{0}$.

Cuando existe una falla en la red la micro red puede operar de forma aislada, entonces las dos micro fuentes tienen que entregar toda la potencia que consume la carga. En la Figura 3.12 se pueden observar los aportes de cada una de las micro fuentes, es decir, la potencia total generada es $P_{t}=P a_{1}+P b_{1}$. En la Figura 3.12 se observan también las potencias 
máximas que pueden entregar las dos fuentes $\left(P b_{\max }\right.$ y $\left.P a_{\max }\right)$ para un valor de frecuencia $\omega_{\min }$ (Chowdhury et al., 2009).

\section{Comentarios acerca de la curva de decaimiento}

Un aspecto interesante de las curvas de decaimiento es que no necesitan un control centralizado o un sistema de comunicación entre las unidades de generación.

El método tradicional de decaimiento no es adecuado cuando se comparten cargas no lineales debido a que los generadores deberían tener en cuenta las corrientes armónicas y a su vez deben controlar la potencia activa y reactiva que aportan. Utilizando el método de decaimiento la potencia compartida es afectada por la impedancia de salida del sistema de generación de energía y la impedancia de línea (Guerrero et al., 2011). Por lo tanto, se han propuesto técnicas de reparto de corriente armónicas para evitar la distorsión de energía que circula al compartir cargas no lineales. Recientemente, se han propuesto técnicas que presentan una impedancia virtual, la cual puede ser vista como otra variable de control. Estas técnicas que ajustan la impedancia de salida de los sistemas de generación de energía eléctrica agregan a la salida inductores o resistores virtuales con el objetivo de compartir la corriente armónica apropiadamente (Guerrero et al., 2011).

\subsection{Retrospección}

A partir de los colapsos de los sistemas eléctricos de potencia hay un interés renovado en la optimización de redes eléctricas y en los sistemas de generación de energía eléctrica. En este sentido existen propuestas como las micro redes. Este nuevo enfoque permite optimizar las pérdidas en la transmisión de energía acercando la generación al centro de consumo y además fomenta la incorporación de sistemas de generación distribuida renovable. Los sistemas de almacenamiento de energía permiten aumentar la confiabilidad y la calidad de la energía para un mercado cada vez más exigente. En este contexto, se presentaron las herramientas para poder estudiar redes eléctricas. Se presentó de una forma simple la transferencia tanto de potencia activa y reactiva en una red. Utilizando este mismo enfoque se presentaron los modelos de cargas estáticas y dinámicas. Las cargas dinámicas son empleadas a menudo en el análisis de una red eléctrica, mientras que en este trabajo de tesis se pretende incorporarlas para modelar el consumo de una carga de una micro red con el objetivo de sintetizar un controlador. Estas expresiones permiten capturar la estructura de un sistema de potencia. En este sentido además de la topología de la red es de interés poder describir las partes constitutivas de la red como funciones de la potencia activa y reactiva que intercambian. Teniendo en cuenta que la mayor potencia generada en una red eléctrica tradicionalmente es utilizando generadores sincrónicos, se presentan las ecuaciones de oscilación empleadas en el análisis de estabilidad de una red. 


\section{Capítulo 4}

\section{Control Vectorial de máquinas doblemente ALIMENTADAS DE ROTOR BOBINADO}

\section{Resumen}

En este capítulo se desarrolla brevemente la motivación y las ideas del control vectorial aplicado a una máquina de inducción, siguiendo a (Vas, 1998) y a (Wu et al., 2011). Se desarrolla el modelo de la máquina de inducción y se plantean las ecuaciones del generador de rotor bobinado. Para el control de la máquina de inducción de rotor bobinado se presentan las técnicas de control vectorial empleando los marcos de referencia de flujo estatórico y según el flujo de red. La nomenclatura para los marcos de referencias utilizados en este trabajo se basa en Vas (1998).

\subsection{Introducción al control vectorial en una máquina de inducción}

Motivados en el trabajo de Park (Park, 1929) del año 1929, en el cual se propuso una nueva forma de analizar una máquina eléctrica, se desarrolló la teoría del control vectorial. La transformación propuesta por Park tiene la propiedad única de eliminar toda inductancia variable en el tiempo de las ecuaciones de tensión de la máquina que se producen debido a los circuitos eléctricos en movimiento relativo y circuitos eléctricos con diferentes reluctancias magnéticas. Actualmente, el desarrollo de la potencia de cálculo y la disponibilidad de diferentes tecnologías asociadas permiten el control de un máquina de inducción eficientemente.

Muchas aplicaciones industriales requieren un control preciso de movimiento. En el pasado 
los motores que cumplían ese rol eran los motores de corriente continua, con el avance de la electrónica de potencia y la capacidad de cálculo de los nuevos microprocesadores estos requerimientos puede cumplirse con máquinas de inducción de corriente alterna. La estructura de control de la máquina de continua es relativamente simple a pesar de que la construcción de la misma es complicada. En la máquina de continua de excitación independiente, el par electromagnético es: (Vas, 1998)

$$
T_{e}=C i_{f} i_{a}=c_{1} \lambda i_{a}
$$

donde $C$ y $c_{1}$ son constantes, $\lambda$ es el flujo de campo, $i_{f}$ es la corriente de flujo e $i_{a}$ es la corriente de armadura. En la ecuación (4.1), asumiendo que el flujo de campo es constante, se observa que el par electromagnético puede variar rápidamente con el cambio de la corriente de armadura. Actualmente las máquinas de inducción dominan el mercado mundial debido a su robustez y simplicidad. Sin embargo, la estructura del control de una máquina de inducción de jaula de ardilla es compleja debida al campo magnético rotante y a la medición de las corrientes del rotor. En sistemas de generación eólica se emplean típicamente generadores de rotor de jaula de ardilla y de rotor bobinado DFIG, (por su sigla en inglés de Doubly-Fed Induction Generator). El DFIG en particular es muy utilizado en aerogeneradores de velocidad variable. Actualmente, esta tecnología ocupa el $50 \%$ del mercado de aerogeneradores (Cardenas et al., 2013). Estos generadores tienen la misma estructura en el estator que los generadores de jaula de ardilla y difieren solamente en la estructura del rotor. El rotor del generador asincrónico de jaula de ardilla es construido empleando un núcleo de hierro laminado y barras conductoras longitudinales. Las láminas están aisladas para minimizar las pérdidas en el hierro. Mientras que un DFIG posee arrollamientos en su rotor, los cuales son alimentados mediante anillos rozantes. Aunque, la máquina de inducción es superior en comparación con la máquina de corriente continua con respecto al tamaño, peso, eficiencia, velocidad máxima, etc, el inconveniente que presenta es que su dinámica es altamente no lineal con una fuerte interacción entre las variables. Por lo tanto, la máquina de inducción requiere un esquema de control más complejo que una de corriente continua. Es posible demostrar que el par electromagnético en una máquina de inducción empleando un marco de referencia de flujo orientado también puede estar representado como el producto de una corriente y un flujo.

Con el objetivo de entender y diseñar accionamientos de par para una máquina de inducción, es necesario conocer el modelo dinámico de la máquina a controlar. A partir de la teoría del vector espacial es posible obtener un modelo válido para la máquina de inducción que contemple variaciones instantáneas de la tensión y de la corriente y que además pueda describir el comportamiento de la máquina en diferentes situaciones de operación (Vas, 1998).

Típicamente, el modelo de la máquina de inducción está compuesto por tres conjuntos de ecuaciones. El primer par de ecuaciones describe las tensiones del estator y del rotor. El segundo par de ecuaciones describe las ecuaciones de flujo en función de las corrientes estatóricas y rotóricas. La última expresión representa las características dinámicas de la velocidad mecánica del rotor en términos del par mecánico y electromagnético (Wu et al., 2011). A continuación se presentan las ecuaciones de la tensión en el estator y en el rotor, el par electromagnético, las ecuaciones fasoriales de la máquina y finalmente la aplicación de un marco de referencia fijo al fasor de flujo estatórico. 


\subsection{Transformación del marco de referencia}

La teoría del marco de referencia puede ser utilizada para simplificar el análisis de máquinas eléctricas y también para facilitar la simulación y la implementación digital de la estrategia de control. Los marcos de referencia comúnmente utilizados son: estacionario, rotórico y sincrónico.

Los marcos de referencia más utilizados son: el marco de referencia " $a b c$ ", el marco de referencia de dos fases $D Q$ y el marco de referencia sincrónico $d q$. A través de una transformación es posible representar un vector en otro marco de referencia. Por ejemplo, las ecuaciones de tensión de una máquina de inducción para cada marco de referencia pueden ser obtenidas asignando la velocidad apropiada $\omega$. Esto es, $\omega=0$ para el marco de referencia estacionario, $\omega=\omega_{r}$ para el marco de referencia en el rotor y $\omega=\omega_{e}$ para el marco de referencia rotante sincrónico. Generalmente, las condiciones de operación determinan el marco de referencia más conveniente para el análisis de un sistema o la simulación (Krause et al., 1995).

\subsubsection{Transformación del marco de referencia $a b c / d q$}

En la Figura 4.1 se considera un fasor genérico y además la relación entre el fasor espacial y el sistema de coordenadas en tres variables $x_{a}, x_{b}$ y $x_{c}$, asumiendo que el módulo del fasor $x$ es constante y que las formas de onda $x_{a}, x_{b}$ y $x_{c}$ son sinusoidales con un desplazamiento de fase de 120 grados (Wu et al., 2011).

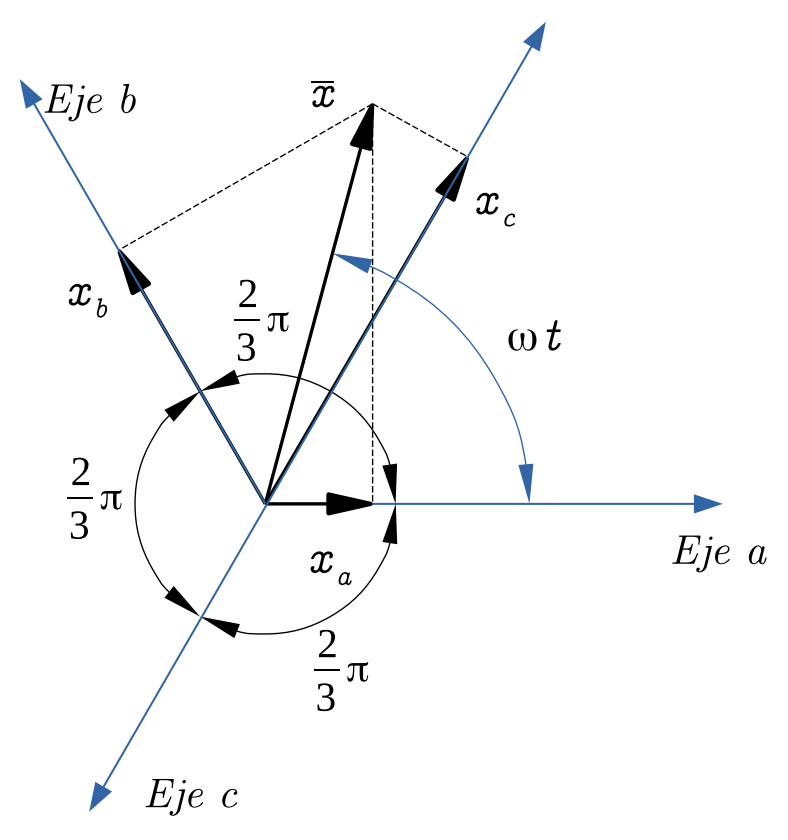

Figura 4.1: Fasor $\bar{x}$ y las tres variables $x_{a}, x_{b}$ y $x_{c}$.

El fasor representado por los vectores $x_{a}, x_{b}$ y $x_{c}$ en el marco de referencia $a b c$, puede ser transformado a un marco de referencia definido como directo $(d)$ y en cuadratura $(q)$. El ángulo $\theta$ relaciona la posición del marco de referencia $d q$, con respecto a los ejes a, b y c del marco estacionario. El marco de referencia $d q$ rota en el espacio a una velocidad $\omega$, definida 
como, $\omega=\frac{d \theta}{d t}$.

Para transformar las variables en el marco estacionario abc al marco de referencia rotante $d q$ se utilizan simples relaciones trigonométricas que involucran la proyección de un vector sobre un eje determinado. En la Figura 4.2 se pueden observar las proyecciones en el eje " $d$ " de las variables $x_{a}, x_{b}$ y $x_{c}$. La suma de todas las proyecciones sobre el eje " $d$ " corresponden al sistema transformado, entonces:

$$
x_{d}=\frac{2}{3}\left[x_{a} \cos (\theta)+x_{b} \cos \left(\theta-\frac{2}{3} \pi\right)+x_{c} \cos \left(\theta-\frac{4}{3} \pi\right)\right] .
$$

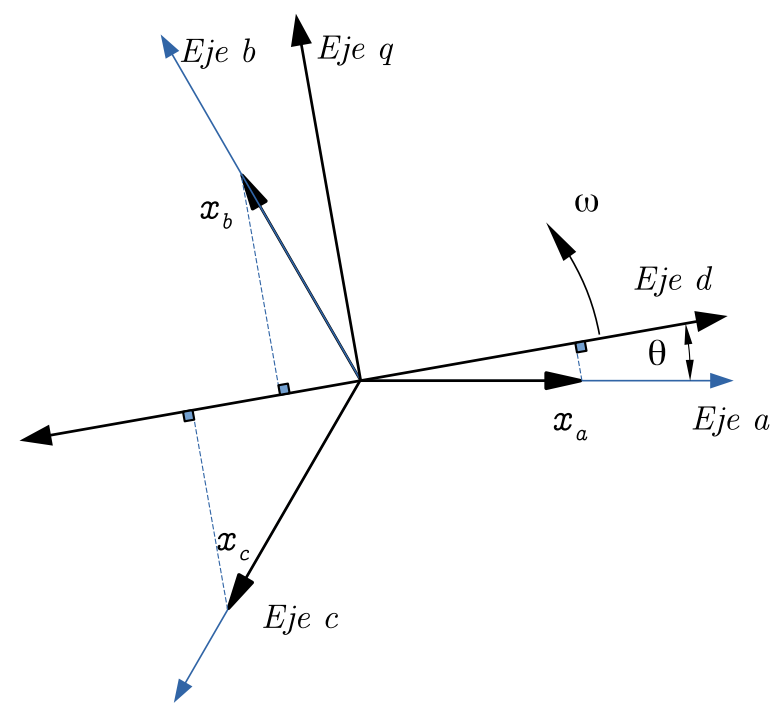

Figura 4.2: Transformación de las variables en el marco de referencia trifásico abc a un marco de referencia $d q$.

Del mismo modo es posible plantear las proyecciones de las variables $x_{a}, x_{b}$ y $x_{c}$ sobre el eje $q$. La transformación de las variables $a b c$ al marco $d q$ pueden expresarse de la siguiente forma:

$$
\left[\begin{array}{l}
x_{d} \\
x_{q}
\end{array}\right]=\frac{2}{3}\left[\begin{array}{ccc}
\cos (\theta) & \cos \left(\theta-\frac{2}{3} \pi\right) & \cos \left(\theta-\frac{4}{3} \pi\right) \\
-\operatorname{sen}(\theta) & -\operatorname{sen}\left(\theta-\frac{2}{3} \pi\right) & -\operatorname{sen}\left(\theta-\frac{4}{3} \pi\right)
\end{array}\right]\left[\begin{array}{l}
x_{a} \\
x_{b} \\
x_{c}
\end{array}\right]
$$

Del mismo modo que para el fasor genérico $x$, es posible representar la relación entre el fasor de tensión de una máquina de inducción en un marco de referencia estacionario representado por

$$
\bar{u}_{s}=\frac{2}{3}\left[u_{s A}(t)+a u_{s B}(t)+a^{2} u_{s C}(t)\right]
$$

donde $a=e^{j \frac{2}{3} \pi}$ y $a^{2}=e^{j \frac{4}{3} \pi}, u_{s A}, u_{s B}$ y $u_{s C}$ son las tensiones instantáneas de fase en el estator con las tensiones $u_{s d}$ y $u_{s q}$ de forma matricial como:

$$
\left[\begin{array}{l}
u_{s d} \\
u_{s q}
\end{array}\right]=\frac{2}{3}\left[\begin{array}{ccc}
\cos (\theta) & \cos \left(\theta-\frac{2}{3} \pi\right) & \cos \left(\theta-\frac{4}{3} \pi\right) \\
-\operatorname{sen}(\theta) & -\operatorname{sen}\left(\theta-\frac{2}{3} \pi\right) & -\operatorname{sen}\left(\theta-\frac{4}{3} \pi\right)
\end{array}\right]\left[\begin{array}{c}
u_{s A} \\
u_{s B} \\
u_{s C}
\end{array}\right]
$$


De la misma forma se puede definir el fasor de tensión en el rotor en el marco de referencia fijo al rotor como:

$$
\bar{u}_{r}=\frac{2}{3}\left[u_{r a}(t)+a u_{r b}(t)+a^{2} u_{r c}(t)\right]=u_{r \alpha}+j u_{r \beta},
$$

donde $u_{r a}(t), u_{r b}(t)$ y $u_{r c}(t)$ son la tensiones instantáneas de fase en el rotor, $u_{r \alpha}$ y $u_{r \beta}$ son las componentes de la tensión en términos de los ejes directos y de los ejes en cuadratura del rotor. Una transformación similar con el objetivo de obtener una representación matricial, expresión (4.5), puede aplicarse a la expresión (4.6).

A su vez existe una transformación inversa que modifica las variables $d q$ en el marco de referencia rotante a las variables $a b c$ en el marco de referencia estacionario. La transformación del marco de referencia dq al marco de referencia estacionario abc puede escribirse de forma matricial de la siguiente forma:

$$
\left[\begin{array}{l}
x_{a} \\
x_{b} \\
x_{c}
\end{array}\right]=\left[\begin{array}{cc}
\cos (\theta) & -\operatorname{sen}(\theta) \\
\cos \left(\theta-\frac{2}{3} \pi\right) & -\operatorname{sen}\left(\theta-\frac{2}{3} \pi\right) \\
\cos \left(\theta-\frac{4}{3} \pi\right) & -\operatorname{sen}\left(\theta-\frac{4}{3} \pi\right)
\end{array}\right]\left[\begin{array}{l}
x_{d} \\
x_{q}
\end{array}\right]
$$

donde:

$$
\begin{gathered}
\omega_{s}=2 \pi f_{s}, \\
\theta_{s}=\int_{0}^{t} \omega_{s}(t) d t+\theta_{0},
\end{gathered}
$$

y $\theta_{0}$ es la posición angular inicial (Wu et al., 2011).

\subsubsection{Transformación del marco de referencia $a b c / D Q$}

Las variables $x_{a}, x_{b}$ y $x_{c}$ en el marco de referencia estacionario pueden transformarse en dos variables en el marco de referencia estacionario $D Q$. Teniendo en cuenta que el marco de referencia $D Q$ no rota en el espacio y empleando la expresión (4.3) se obtiene la siguiente forma matricial:

$$
\left[\begin{array}{l}
x_{D} \\
x_{Q}
\end{array}\right]=\frac{2}{3}\left[\begin{array}{ccc}
1 & -\frac{1}{2} & -\frac{1}{2} \\
0 & \frac{\sqrt{3}}{2} & -\frac{\sqrt{3}}{2}
\end{array}\right]\left[\begin{array}{l}
x_{a} \\
x_{b} \\
x_{c}
\end{array}\right] .
$$

De forma similar la transformación inversa puede escribirse de la siguiente forma:

$$
\left[\begin{array}{l}
x_{a} \\
x_{b} \\
x_{c}
\end{array}\right]=\left[\begin{array}{cc}
1 & 0 \\
-\frac{1}{2} & -\frac{\sqrt{3}}{2} \\
-\frac{1}{2} & -\frac{\sqrt{3}}{2}
\end{array}\right]\left[\begin{array}{l}
x_{D} \\
x_{Q}
\end{array}\right]
$$

\subsubsection{Par electromagnético en una máquina de inducción de corriente alterna}

Como ya se comentó, el par electromagnético es de interés para analizar el problema del control de la máquina de inducción. A continuación se desarrollan las expresiones para el 
mismo mediante la rapidez de cambio de energía mecánica $\left(\frac{d W_{m e c}}{d t}\right)$ e igualándola a la potencia mecánica de salida de la máquina $\left(P_{m e c}\right)$ de la siguiente forma:

$$
\frac{d W_{m e c}}{d t}=P_{m e c}=T_{e} \omega_{r},
$$

donde $\omega_{r}$ es la velocidad de giro del eje del rotor, definida como $\omega_{r}=\frac{d \theta_{r}}{d t}$. En la ecuación (4.12), por simplicidad es considerada una máquina de dos polos. Dado que el principio de conservación de la energía debe cumplirse, la energía eléctrica provista a la máquina debe cubrir las pérdidas en el estator y en el rotor $\left(W_{l}\right)$, la energía almacenada en el campo magnético $\left(W_{m a g}\right)$ y la energía mecánica de salida $\left(W_{m e c}\right)$ es decir:

$$
W_{e}=W_{l}+W_{m a g}+W_{m e c} .
$$

Además, la derivada de la ecuación anterior es la potencia mecánica de salida y está dada por:

$$
\dot{W}_{m e c}=\dot{W}_{e}-\dot{W}_{l}-\dot{W}_{m a g}
$$

Las tres componentes de la ecuación anterior son desarrolladas a continuación para una máquina polifásica doblemente alimentada.

La tasa de cambio de la energía eléctrica en la máquina es la suma de la potencia instantánea en el rotor y en el estator. La potencia en el estator se puede escribir como:

$$
P_{s}=\frac{3}{2} \operatorname{Re}\left(\bar{u}_{s} \bar{i}_{s}^{*}\right)
$$

donde $\bar{u}_{s}$ e $\bar{i}_{s}^{*}$ son los fasores espaciales de tensión y de corriente en el estator expresados en un marco de referencia estacionario. El asterisco en la expresión anterior representa el complejo conjugado. La potencia en el rotor es:

$$
P_{r}=\frac{3}{2} \operatorname{Re}\left(\bar{u}_{r}^{\prime} \bar{i}_{r}^{* *}\right)
$$

donde $\bar{u}_{r}^{\prime}$ e $\bar{i}_{r}^{\prime}$ son los fasores espaciales de tensión y corriente en el rotor en un marco de referencia fijo al estator.

De esta forma la tasa de cambio de la energía eléctrica, está dada por:

$$
\frac{d W_{e}}{d t}=P_{s}+P_{r}=\frac{3}{2} \operatorname{Re}\left(\bar{u}_{s} \bar{i}_{s}^{*}+\bar{u}_{r}^{\prime} \bar{i}_{r}^{*}\right) .
$$

Considerando solamente las pérdidas resistivas en los arrollamientos del estator y del rotor, entonces la tasa de cambio de $W_{l}$ queda definida como:

$$
\frac{d W_{l}}{d t}=\frac{3}{2}\left(R_{s}\left|\bar{i}_{s}\right|^{2}+R_{r}\left|\bar{i}_{r}\right|^{2}\right)
$$

donde $R_{s}$ y $R_{r}$ son los valores de la resistencia estatórica y rotórica, respectivamente.

Por último, la tasa de variación de la energía almacenada en el campo magnético se puede escribir como:

$$
\frac{d W_{m a g}}{d t}=\frac{3}{2} \operatorname{Re}\left(\bar{u}_{s i} \bar{i}_{s}^{*}+\bar{u}_{r i}^{\prime} i_{r}^{*}\right)
$$


donde $\bar{u}_{s i}$ y $\bar{u}_{r i}^{\prime}$ son los fasores de la tensiones inducidas (f.e.m.i.) en el estator y en el rotor, respectivamente. Ambas están expresadas en un marco de referencia fijo al estator. La f.e.m.i. en el estator $\left(\bar{u}_{s i}\right)$ es definida como:

$$
\bar{u}_{s i}=\frac{d \bar{\lambda}_{s}}{d t}
$$

donde $\bar{\lambda}_{s}$ es el flujo de acoplamiento estatórico dado por:

$$
\bar{\lambda}_{s}=L_{s} \bar{i}_{s}+L_{m} \bar{i}_{r}^{\prime}=L_{s} \bar{i}_{s}+L_{m} \bar{i}_{r} e^{j \theta_{r}}
$$

donde $L_{s}$ es la inductancia total de las tres fases del estator, $L_{m}$ es la inductancia magnetizante. El fasor de acoplamiento de flujo $\left(\bar{\lambda}_{s}\right)$ describe el módulo y el ángulo de fase del pico de la distribución sinusoidal de flujo del entrehierro.

En la expresión (4.21) $L_{s} \bar{i}_{s}$ es el fasor espacial de flujo de acoplamiento propio de las fases del estator causado por las corrientes en el estator. $L_{m} \bar{i}_{r}^{\prime}$ es el fasor espacial de acoplamiento mutuo, el cual es debido a las corrientes rotóricas y es expresado en un marco de referencia estacionario.

La f.e.m.i. en el rotor $\left(\bar{u}_{r i}\right)$ es debida a la tasa de variación de flujo de acoplamiento en el rotor, dada por:

$$
\bar{u}_{r i}=\frac{d \bar{\lambda}_{r}^{\prime}}{d t}
$$

donde $\bar{\lambda}_{r}^{\prime}$ es el flujo de acoplamiento en el rotor en el marco de referencia estacionario.

El flujo de acoplamiento en el rotor se define como:

$$
\bar{\lambda}_{r}=L_{r} \bar{i}_{r}+L_{m} \bar{i}_{s}^{\prime},
$$

con $L_{r}$ la inductancia rotórica trifásica e $\bar{i}_{s}^{\prime}$ el fasor de la corriente del estator expresado en el marco de referencia del rotor. A su vez el flujo de acoplamiento en el rotor en el marco de referencia estacionario está dado por:

$$
\bar{\lambda}_{r}^{\prime}=L_{r} \bar{i}_{r}^{\prime}+L_{m} \bar{i}_{s} .
$$

La ecuación de la tasa de variación de la energía del campo magnético (4.19) puede ser reescrita con las definiciones de la f.e.m.i. en el estator (4.20) y en el rotor (4.22) de la siguiente manera:

$$
\frac{d W_{m a g}}{d t}=\frac{3}{2} \operatorname{Re}\left(\frac{d \bar{\lambda}_{s}}{d t} \bar{i}_{s}^{*}+\frac{d \bar{\lambda}_{r}^{\prime}}{d t} \bar{i}_{r}^{*}\right),
$$

entonces, $W_{\text {mag }}$ puede ser expresado como:

$$
W_{\text {mag }}=\frac{3}{2} \operatorname{Re}\left(\bar{i}_{s}^{*} \bar{\lambda}_{s}+\bar{i}_{r}^{*} \bar{\lambda}_{r}^{\prime}\right),
$$

La potencia mecánica puede escribirse considerando la potencia desarrollada en el estator y en el rotor de la siguiente forma:

$$
\dot{W}_{m e c}=\dot{W}_{m e c s}+\dot{W}_{m e c r} .
$$


Además, aplicando las expresiones de la tasa de variación de la energía eléctrica $\left(\dot{W}_{e}\right)$, las pérdidas eléctricas $\left(\dot{W}_{l}\right)$ y la energía en el campo $\left(\dot{W}_{l}\right)$ se puede escribir la potencia mecánica en el estator y en el rotor como:

$$
\dot{W}_{\text {mecs }}=\frac{3}{2}\left[\operatorname{Re}\left(\bar{u}_{s} \bar{i}_{s}^{*}\right)-R_{s}\left|\bar{i}_{s}\right|^{2}-\operatorname{Re}\left(\bar{i}_{s}^{*} \frac{d \bar{\lambda}_{s}}{d t}\right)\right],
$$

y

$$
\dot{W}_{\text {mecr }}=\frac{3}{2}\left[\operatorname{Re}\left(\bar{u}_{r}^{\prime} \bar{i}_{r}^{\prime *}\right)-R_{r}\left|\bar{i}_{r}^{\prime}\right|^{2}-\operatorname{Re}\left(\bar{i}_{r}^{\prime *} \frac{d \lambda_{r}^{\prime}}{d t}\right)\right] .
$$

Desde un marco de referencia estacionario, el fasor de tensión del estator $\left(\bar{u}_{s}\right)$ puede estar solo balanceado por $\left(R_{s} \bar{i}_{s}\right)$ y $\frac{d \bar{\lambda}_{s}}{d t}$, entonces, $\dot{W}_{\text {mecs }}=0$. Empleando las consideraciones previas el fasor de la tensión en el rotor $\left(\bar{u}_{r}^{\prime}\right)$ debe ser balanceado por la suma de la caída de tensión en la resistencia óhmica del rotor $\left(R_{r} \bar{i}_{r}^{\prime}\right)$ más la tasa de cambio del flujo de acoplamiento $\left(\frac{d \lambda_{r}^{\prime}}{d t}\right)$ y una tensión rotacional $-j \omega_{r} \bar{\lambda}_{r}^{\prime}$. De esta forma, se puede escribir una expresión para la potencia mecánica de la siguiente forma:

$$
P_{m e c}=\frac{3}{2} \operatorname{Re}\left(j \omega_{r} \bar{\lambda}_{r}^{\prime} \bar{i}_{r}^{*}\right)=\frac{3}{2} \omega_{r} \operatorname{Re}\left(j \bar{\lambda}_{r}^{\prime} \bar{i}_{r}^{*}\right)=-\frac{3}{2} \omega_{r} \bar{\lambda}_{r}^{\prime} \times \bar{i}_{r}^{\prime} .
$$

Entonces, de acuerdo a la expresión anterior, la potencia mecánica es proporcional a la velocidad del rotor y al producto vectorial entre el flujo rotórico y el fasor de corriente en el rotor.

La expresión para el par electromagnético para una máquina con un par de polos puede escribirse utilizando la expresión (4.12) y la definición previa de la potencia mecánica de la siguiente forma:

$$
T_{e}=\frac{P_{m e c}}{\omega_{r}}=-\frac{3}{2} \bar{\lambda}_{r}^{\prime} \times \bar{i}_{r}^{\prime} .
$$

La expresión anterior del par electromagnético puede ser reescrita reemplazando $\lambda_{r}^{\prime}$ por la expresión (4.24), como:

$$
T_{e}=-\frac{3}{2}\left(L_{r} \bar{i}_{r}^{\prime}+L_{m} \bar{i}_{s}\right) \times \bar{i}_{r}^{\prime},
$$

donde $L_{r}$ y $L_{m}$ son la inductancia rotórica y la inductancia magnetizante de la máquina, respectivamente. Si se desarrolla el producto vectorial en la expresión anterior, se puede observar que uno de los términos queda como $\left(\bar{i}_{r}^{\prime} \times \bar{i}_{r}^{\prime}=0\right)$. Esta afirmación es simple de verificar calculando el módulo del vector resultante del producto vectorial. Entonces, la expresión (4.32) puede escribirse como:

$$
T_{e}=-\frac{3}{2} L_{m} \bar{i}_{s} \times \bar{i}_{r}^{\prime}
$$

Como se comentó previamente, el flujo de acoplamiento estatórico puede representarse como $\bar{\lambda}_{s}=L_{s} \bar{i}_{s}+L_{m} \bar{i}_{r}^{\prime}$, entonces el par electromagnético está dado por:

$$
T_{e}=-\frac{3 L_{m}}{2 L_{s}} \bar{\lambda}_{s} \times \bar{i}_{r}^{\prime}
$$

donde $L_{s}$ es la inductancia estatórica de la máquina de inducción. Cabe señalar que las expresiones generales desarrolladas derivadas de valores instantáneos del valor del par electromagnético son válidas para máquinas simétricas sin restricción alguna sobre la variación temporal de las corrientes del rotor y del estator. Además, se asume que tanto la distribución espacial de la densidad de flujo como la densidad de corriente son sinusoidales. 


\section{Diferentes expresiones para el par electromagnético}

Es posible escribir el par electromagnético y la potencia mecánica dada por la expresión (4.12) en un marco de referencia general de la siguiente forma:

$$
T_{e}=-\frac{3}{2} \bar{\lambda}_{r} \times \bar{i}_{r}^{\prime}=-\frac{3}{2} \bar{\lambda}_{r g} e^{j \theta_{g}} \times \bar{i}_{r g} e^{j \theta_{g}}=-\frac{3}{2} \bar{\lambda}_{r g} \times \bar{i}_{r g},
$$

$\mathrm{y}$

$$
P_{m e c}=-\frac{3}{2} \omega_{r} \bar{\lambda}_{r g} \times \bar{i}_{r g}
$$

Para una máquina de inducción con "np" pares de polos, el par electromagnético de acuerdo a la interacción entre los fasores del flujo rotórico y la corriente en el rotor en un marco de referencia general es:

$$
T_{e}=-\frac{3}{2} n p \bar{\lambda}_{r g} \times \bar{i}_{r g} .
$$

De la misma forma la ecuación que representa el par electromagnético como una interacción entre los fasores de las corrientes en el estator y el rotor (4.33) en un marco general puede ser escrita como:

$$
T_{e}=-\frac{3}{2} n p L_{m} \bar{i}_{s g} \times \bar{i}_{r g} .
$$

Aplicando el principio de acción y reacción es posible reescribir la ecuación del par electromagnético (4.37) como:

$$
T_{e}=\frac{3}{2} n p \bar{\lambda}_{s g} \times \bar{i}_{s g} .
$$

Otra forma de llegar a la expresión anterior puede ser considerando que el flujo estatórico en un marco de referencia general $\left(\bar{\lambda}_{s g}\right)$ está definido como

$$
\bar{\lambda}_{s g}=L_{s} \bar{i}_{s g}+L_{m} \bar{i}_{r g},
$$

y que el par electromagnético puede escribirse como $T_{e}=\frac{3}{2} n p \bar{\lambda}_{s g} \times \bar{i}_{s g}$, por último como el producto vectorial de dos vectores coincidentes es cero, es decir $\bar{i}_{s g} \times \bar{i}_{s g}=0$ se demuestra la expresión (4.39).

\subsubsection{Ecuaciones fasoriales de la máquina}

\section{Ecuaciones de tensión en diferentes marcos de referencia}

Las ecuaciones de los fasores de tensión en una máquina de inducción en un marco de referencia estacionario son presentados a continuación. Los fasores de tensión en el estator y en el rotor son:

$\mathrm{y}$

$$
\bar{u}_{s}=R_{s} \bar{i}_{s}+\frac{d \bar{\lambda}_{s}}{d t}
$$

$$
\bar{u}_{r}^{\prime}=R_{r} \bar{i}_{r}^{\prime}+\frac{d \bar{\lambda}_{r}^{\prime}}{d t}-j \omega_{r} \bar{\lambda}_{r}^{\prime}
$$

donde $\omega_{r}$ es la velocidad angular del rotor, además los flujos $\bar{\lambda}_{s}$ y $\bar{\lambda}_{r}$ están definidos en términos de la inductancia de la máquina de inducción y los fasores de las corrientes estatóricas y rotóricas. El primer término de las expresiones (4.41) y (4.42) representa las cargas óhmicas 
en el estator y en el rotor, respectivamente. El segundo término es un transformador de f.e.m., representado por la derivada del fasor de flujo estatórico y rotórico, respectivamente. El termino $-j \omega_{r} \bar{\lambda}_{r}^{\prime}$ representa una conversión electromecánica de la energía. Por último, en la expresión (4.42), $\bar{u}_{r}^{\prime}$ representa la tensión en el rotor referida a un marco de referencia estacionario.

Utilizando las definiciones de los flujos de acoplamiento estatórico y rotórico en el marco de referencia fijo al estator es posible escribir las expresiones de la tensión en el estator y en el rotor de la siguiente forma:

$$
\left[\begin{array}{c}
\bar{u}_{s} \\
\bar{u}_{r}^{\prime}
\end{array}\right]=\left[\begin{array}{cc}
R_{s} & 0 \\
0 & R_{r}
\end{array}\right]\left[\begin{array}{c}
\bar{i}_{s} \\
\bar{i}_{r}^{\prime}
\end{array}\right]+\frac{d}{d t}\left[\begin{array}{cc}
L_{s} & L_{m} \\
L_{m} & L_{r}
\end{array}\right]\left[\begin{array}{c}
\bar{i}_{s} \\
\bar{i}_{r}^{\prime}
\end{array}\right]-j \omega_{r}\left[\begin{array}{cc}
0 & 0 \\
L_{m} & L_{r}
\end{array}\right]\left[\begin{array}{c}
\bar{i}_{s} \\
\bar{i}_{r}^{\prime}
\end{array}\right] .
$$

Esta expresión a diferencia de un modelo trifásico es mucho más simple.

A partir de las siguientes relaciones fasoriales de la tensión y la corriente del rotor en un marco de referencia fijo al rotor

$$
\begin{gathered}
\bar{u}_{r}=\bar{u}_{r}^{\prime} e^{-j \theta_{r}}, \\
\bar{i}_{r}=\bar{i}_{r}^{\prime} e^{-j \theta_{r}},
\end{gathered}
$$

es posible reescribir la ecuación (4.43) de la siguiente forma:

$$
\left[\begin{array}{c}
\bar{u}_{s} \\
\bar{u}_{r}
\end{array}\right]=\left[\begin{array}{cc}
R_{s} & 0 \\
0 & R_{r}
\end{array}\right]\left[\begin{array}{l}
\bar{i}_{s} \\
\bar{i}_{r}
\end{array}\right]+\frac{d}{d t}\left[\begin{array}{cc}
L_{s} & L_{m} e^{j \theta_{r}} \\
L_{m} e^{-j \theta_{r}} & L_{r}
\end{array}\right]\left[\begin{array}{c}
\bar{i}_{s} \\
\bar{i}_{r}
\end{array}\right] .
$$

\subsubsection{Aplicación de un marco de referencia fijo al fasor de flujo estatórico}

Si un marco de referencia fijo al flujo estatórico es utilizado, donde la componente en cuadratura es nula, tanto en el transitorio como en estado estacionario, el par electromagnético desarrollado en una máquina de inducción con $n p$ pares de polos es:

$$
T_{e}=n p \frac{3}{2} \lambda_{s x} i_{s y},
$$

donde $\lambda_{s x}$ es el flujo estatórico en el eje real del marco de referencia especial e $i_{s y}$ es la componente imaginaria de la corriente en el estator en el mismo marco de referencia.

En la Figura 4.3 se muestra la relación entre el marco de referencia especial y el marco de referencia estacionario. En esta figura se puede observar que $\rho_{s}$ representa la posición de la corriente magnetizante con respecto al eje real $D$ del marco de referencia estacionario.

El fasor de flujo del estator puede ser expresado de la siguiente forma:

$$
\bar{\lambda}_{s}=\lambda_{s D}+j \lambda_{s Q}=\left|\bar{\lambda}_{s}\right| e^{j \rho_{s}} .
$$

La velocidad del marco especial de referencia está dada por:

$$
\omega=\frac{d \rho_{s}}{d t} .
$$




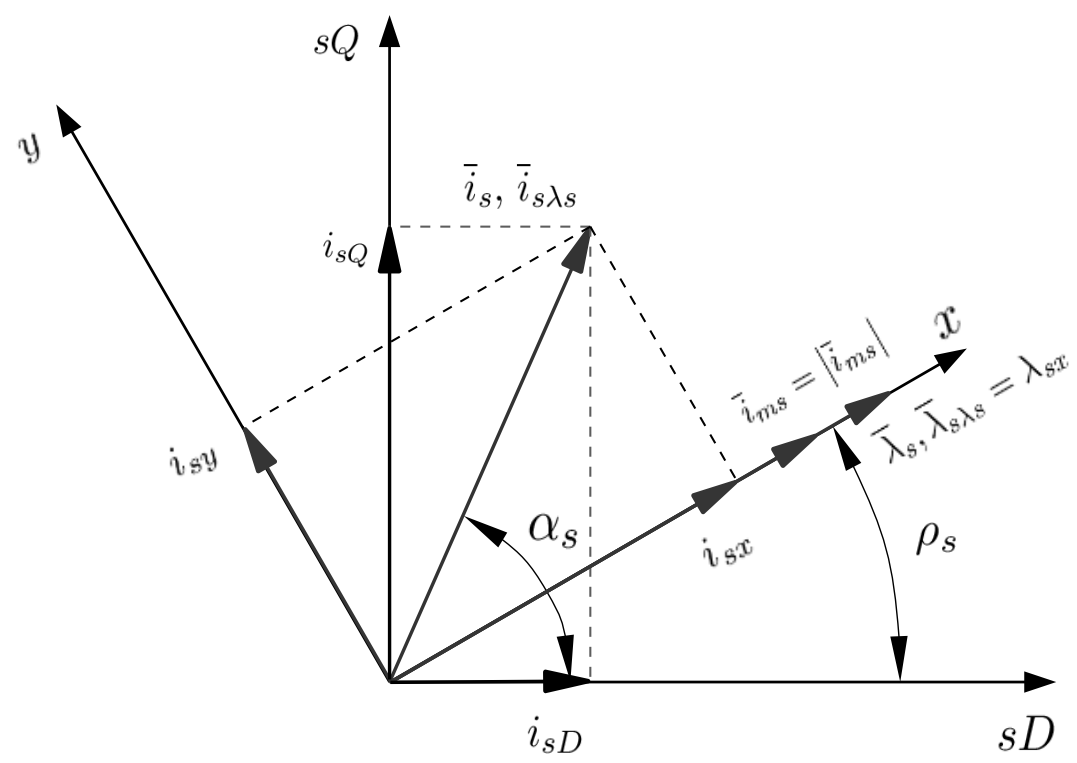

Figura 4.3: Relación entre el marco de referencia especial fijo al estator y el marco de referencia estacionario.

De esta manera, en el marco de referencia especial el fasor de corriente y el fasor de flujo son:

$$
\bar{i}_{s \lambda s}=i_{s x}+j i_{s y}=\bar{i}_{s} e^{-j \rho_{s}}=\left(i_{s D}+j i_{s Q}\right) e^{-j \rho_{s}}=\left(i_{s D}+j i_{s Q}\right)\left(\cos \left(\rho_{s}\right)-j \operatorname{sen}\left(\rho_{s}\right)\right),
$$

y

$$
\bar{\lambda}_{s \lambda s}=\bar{\lambda}_{s} e^{-j \rho_{s}}=\left(\lambda_{s D}+j \lambda_{s Q}\right) e^{-j \rho_{s}}=\lambda_{s x}+j \lambda_{s y} .
$$

Si se reemplaza la expresión (4.48) en la ecuación anterior se puede obtener:

$$
\bar{\lambda}_{s \lambda s}=\left|\bar{\lambda}_{s}\right| e^{j \rho_{s}} e^{-j \rho_{s}}=\left(\lambda_{s D}+j \lambda_{s Q}\right) e^{-j \rho_{s}} \Rightarrow\left|\bar{\lambda}_{s}\right|=\lambda_{s x}+j 0 .
$$

Si se desarrolla el producto de la ecuación (4.50) se obtiene la siguiente representación matricial de la transformación de rotación:

$$
\left[\begin{array}{l}
i_{s x} \\
i_{s y}
\end{array}\right]=\left[\begin{array}{cc}
\cos \left(\rho_{s}\right) & \operatorname{sen}\left(\rho_{s}\right) \\
-\operatorname{sen}\left(\rho_{s}\right) & \cos \left(\rho_{s}\right)
\end{array}\right]\left[\begin{array}{c}
i_{s D} \\
i_{s Q}
\end{array}\right],
$$

la cual representa la relación entre las corrientes $i_{s D}, i_{s Q}$ y las corrientes en el eje $x$ e $y$ $\left(i_{s x}\right.$ e $\left.i_{s y}\right)$. Es posible escribir el par electromagnético de forma tal que el flujo del estator es expresado en términos de las corrientes del estator y del rotor de la siguiente forma:

$$
\bar{\lambda}_{s \lambda s}=L_{s} \bar{i}_{s \lambda s}+L_{m} \bar{i}_{r \lambda s},
$$

donde $\bar{i}_{s \lambda s}$ está definida en la expresión (4.50), teniendo en cuenta que $\theta_{g}=\rho_{s}, \bar{i}_{r \lambda s}$ está definido como:

$$
\bar{i}_{r \lambda s}=i_{r x}+j i_{r y}=\bar{i}_{r} e^{-j\left(\rho_{s}-\theta_{r}\right)}=\bar{i}_{r} e^{j \theta_{r}} e^{-j \rho_{s}},
$$

en el marco de referencia fijo al estator $\bar{i}_{r}^{\prime}=\bar{i}_{r} e^{j \theta_{r}}$, reemplazando esta definición en la expresión anterior se obtiene:

$$
\bar{i}_{r \lambda s}=\bar{i}_{r}^{\prime} e^{-j \rho_{s}}=\left(i_{r d}+j i_{r q}\right) e^{-j \rho_{s}},
$$


donde $\bar{i}_{r}$ es el fasor de corriente en el marco de referencia del rotor e $\bar{i}_{r}^{\prime}$ es el fasor de corriente en el rotor en el marco de referencia estacionario fijo al estator. Luego se puede definir la corriente magnetizante $\bar{i}_{m s}$ en el marco especial de referencia que rota a la velocidad $\omega_{m s}$ como:

$$
\bar{i}_{m s}=i_{m s x}+j i_{m s y}=i_{m s x}=\left|\bar{i}_{m s}\right|=\frac{\lambda_{s x}}{L_{m}}=\bar{i}_{s \lambda s}\left(1+\sigma_{s}\right)+\bar{i}_{r \lambda s},
$$

con $\sigma_{s}$ un factor de pérdida del estator. Finalmente, el par electromagnético puede representarse como:

$$
T_{e}=\frac{3}{2} n p L_{m}\left|\bar{i}_{m s}\right| i_{s y}
$$

donde $\left|\bar{i}_{m s}\right|$ es la corriente que produce el flujo e $i_{s y}$ es la componente de la corriente del estator que produce el par electromagnético. De esta forma, se puede observar de la ecuación anterior que de la misma manera que en la máquina de corriente continua, en la máquina de inducción el par puede ser controlado a través de estas dos corrientes. Además, en estado estacionario estas dos corrientes son valores constantes.

\subsection{Control de la máquina de inducción de rotor bobinado (DFIG)}

En la Figura 4.4 se puede observar el esquema de la conexión eléctrica de un generador doblemente alimentado (DFIG) a una red. El estator del generador está directamente conectado a la red eléctrica mientras que el rotor se conecta mediante un convertidor espalda contra espalda que procesa una porción de la potencia total del generador. El convertidor

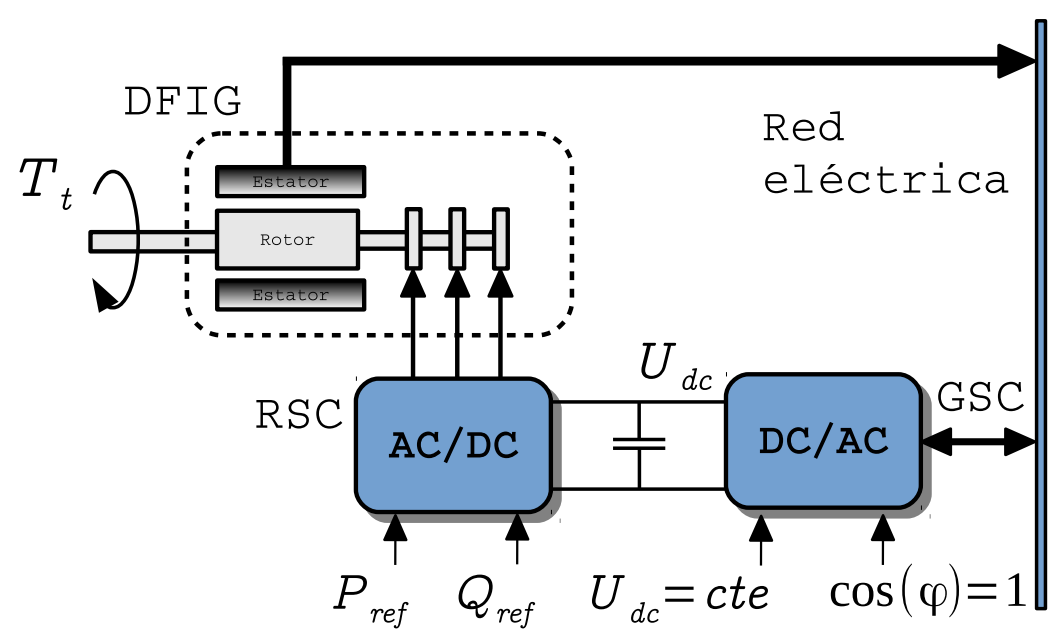

Figura 4.4: Esquema de conexión de un generador de inducción doblemente alimentado (DFIG).

espalda contra espalda está compuesto por dos convertidores de tensión VSC, (por su sigla en inglés Voltage Source Converter), uno de ellos está conectado a la red GSC, (por su sigla en inglés Grid Side Converter) y el otro convertidor está conectado al rotor de la máquina RSC, (por su sigla en inglés Rotor Side Converter). El control de la máquina se realiza a través del RSC empleando control vectorial, mientras que la finalidad del convertidor del lado de la red es la de garantizar las condiciones para el intercambio de potencia con la red. En particular, el control del GSC tiene como objetivo mantener la tensión del bus de continua 
$\left(U_{d c}\right)$ constante. Además, es frecuente considerar que el GSC presente un factor de potencia unitario al resto del sistema eléctrico, como se indica en la Figura 4.4. Los convertidores permiten que el sistema opere a velocidad variable, desvinculando la frecuencia del sistema eléctrico y la frecuencia mecánica del rotor.

\subsubsection{Control por flujo estatórico}

Como se observa en la expresión (4.58) el par electromagnético puede ser controlado a través de la corriente $\left(i_{s y}\right)$, la cual es la responsable de producir el momento electromagnético. En la expresión (4.58) el fasor de la corriente magnetizante puede escribirse en términos de los fasores de corriente del estator y del rotor expresados en términos del marco de referencia del flujo estatórico orientado $\left(\bar{i}_{s \lambda s}, \bar{i}_{r \lambda s}\right)$.

$$
\left|\bar{i}_{m s}\right|=\bar{i}_{r \lambda s}+\frac{\bar{i}_{s \lambda s} L_{s}}{L_{m}}=\left(i_{r x}+j i_{r y}\right)+\left(i_{s x}+j i_{s y}\right) \frac{L_{s}}{L_{m}},
$$

donde $i_{r x}, i_{r y}, i_{s x}$ e $i_{s y}$ son las componentes de los fasores de las corrientes del rotor y de estator, respectivamente. Estas corrientes están expresadas en el marco de referencia de flujo estatórico.

Para un mejor entendimiento, el fasor de la corriente magnetizante y el fasor de la corriente rotórica son graficados en la Figura 4.5. En la misma está superpuesto el nuevo marco de referencia $(x, y)$ y además se indican los ángulos $\alpha_{r}, \theta_{r}$ y $\rho_{s}$. El ángulo del fasor de la corriente magnetizante se indica como " $\phi_{r}$ " y está dado por:

$$
\phi_{r}=\alpha_{r}+\theta_{r}-\rho_{s}
$$

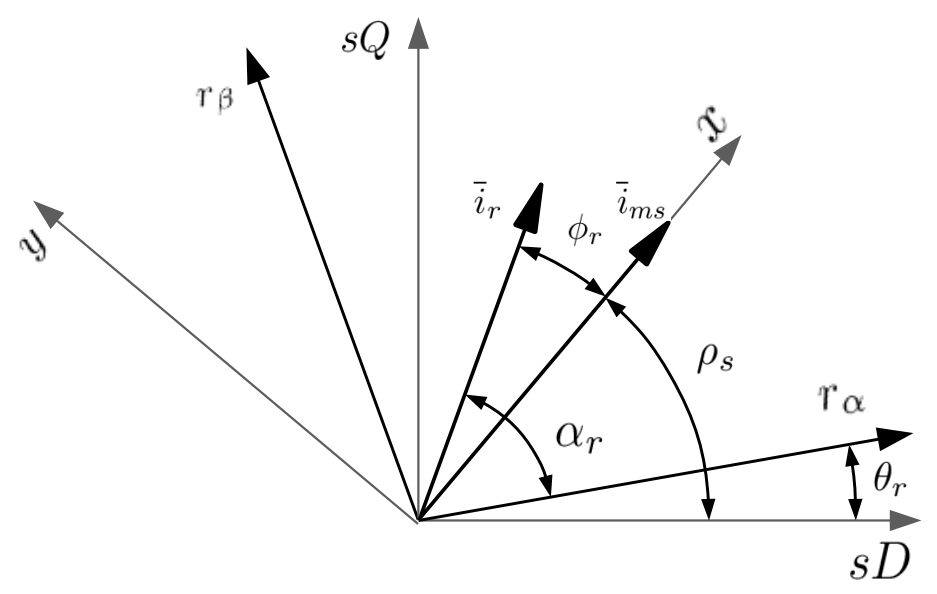

Figura 4.5: Fasor de la corriente magnetizante del estator y fasor de la corriente del rotor.

Una nueva expresión para el par electromagnético puede ser obtenida reescribiendo la corriente, $\left(i_{s y}\right)$, que lo produce de la siguiente forma:

$$
i_{s y}=-\frac{L_{m}}{L_{s}} i_{r y}
$$

siendo $i_{r y}$ la proyección del fasor de corriente en el rotor sobre el eje " $y$ ", dado por:

$$
i_{r y}=\left|\bar{i}_{r}\right| \operatorname{sen}\left(\phi_{r}\right) \text {. }
$$


Entonces, reemplazando la ecuación anterior (4.62) en la expresión de la corriente en el estator en el eje " $y$ " (4.61) se obtiene:

$$
i_{s y}=-\frac{L_{m}}{L_{s}}\left|\bar{i}_{r}\right| \operatorname{sen}\left(\phi_{r}\right) .
$$

Esta última expresión se puede reemplazar en la expresión del par electromagnético de la siguiente forma:

$$
T_{e}=-c\left|\bar{i}_{m s}\right| \frac{L_{m}}{L_{s}}\left|\bar{i}_{r}\right| \operatorname{sen}\left(\phi_{r}\right)
$$

\subsubsection{Tensión estatórica para una máquina de rotor bobinado}

Con el objetivo de escribir las ecuaciones de tensión en el estator para un DFIG es útil expresar $\bar{i}_{s \lambda s}$ en términos de $\left|\bar{i}_{m s}\right|$ e $\bar{i}_{r \lambda s}$. Entonces, a partir de la expresión de la corriente magnetizante definida en la expresión (4.59) se obtiene:

$$
\bar{i}_{s \lambda s}=\frac{L_{m}}{L_{s}}\left(\left|\bar{i}_{m s}\right|-\bar{i}_{r \lambda s}\right) .
$$

La tensión en el estator de una máquina de inducción es:

$$
\bar{u}_{s \lambda s}=R_{s} \bar{i}_{s \lambda s}+L_{s} \frac{d \bar{i}_{s \lambda s}}{d t}+L_{m} \frac{d \bar{i}_{r \lambda s}}{d t}+j \omega L_{s} \bar{i}_{s \lambda s}+j \omega L_{m} \bar{i}_{r \lambda s}
$$

con

$$
\omega=\frac{d \rho_{s}}{d t}
$$

La tensión en el estator en el mismo marco de referencia puede ser expresada como:

$$
\bar{u}_{s \lambda s}=u_{s x}+j u_{s y}=\left(u_{s D}+j u_{s Q}\right) e^{-j \rho_{s}}=\bar{u}_{s} e^{-j \rho_{s}} .
$$

Si se reemplaza en la expresión de la tensión en el estator (4.66) con la expresión de $\bar{i}_{s \lambda s}$ (4.65) se obtiene la tensión estatórica en un DFIG de la siguiente forma:

$$
\bar{u}_{s \lambda s}=\frac{R_{s} L_{m}}{L_{s}}\left(\left|\bar{i}_{m s}\right|-\bar{i}_{r \lambda s}\right)+L_{m} \frac{d\left|\bar{i}_{m s}\right|}{d t}+j \omega L_{m}\left|\bar{i}_{m s}\right| .
$$

Esta última expresión puede reescribirse de la siguiente forma:

$$
T_{s} \frac{d\left|\bar{i}_{m s}\right|}{d t}+\left|\bar{i}_{m s}\right|\left(1+j \omega T_{s}\right)=\bar{u}_{s \lambda s}\left(\frac{L_{s}}{R_{s} L_{m}}\right)+\bar{i}_{r \lambda s}
$$

donde $T_{s}=\frac{L_{s}}{R_{s}}$. La ecuación anterior (4.69) se puede descomponer en la parte real e imaginaria de la siguiente forma:

$$
T_{s} \frac{d\left|\bar{i}_{m s}\right|}{d t}+\left|\bar{i}_{m s}\right|=u_{s x}\left(\frac{L_{s}}{R_{s} L_{m}}\right)+i_{r x}
$$

y la parte imaginaria es:

$$
\omega T_{s}\left|\bar{i}_{m s}\right|=u_{s y}\left(\frac{L_{s}}{R_{s} L_{m}}\right)+i_{r y} .
$$

Las componentes de tensión del estator para una máquina de rotor bobinado pueden ser desarrolladas asumiendo una tensión trifásica simétrica con una frecuencia $\omega_{1}$. El fasor de tensión en el estator en el marco de referencia estacionario es:

$$
\bar{u}_{s}=\sqrt{2} U_{s} e^{j \omega_{1} t},
$$


donde $U_{s}$ es el valor r.m.s. de la tensión de línea al neutro. Entonces, sustituyendo la expresión anterior en (4.67) se obtiene:

$$
\bar{u}_{s \lambda s}=\bar{u}_{s} e^{-j \rho_{s}}=\sqrt{2} U_{s} e^{j\left(\omega_{1} t-\rho_{s}\right)}=u_{s x}+j u_{s y} .
$$

\subsubsection{Tensión rotórica para una máquina de rotor bobinado}

Aplicando un marco de referencia orientado según el flujo estatórico, el fasor de tensión en el rotor es:

$$
\bar{u}_{r \lambda s}=R_{r} \bar{i}_{r \lambda s}+L_{r} \frac{d \bar{i}_{r \lambda s}}{d t}+L_{m} \frac{d \bar{i}_{s \lambda s}}{d t}+j\left(\omega-\omega_{r}\right)\left(L_{r} \bar{i}_{r \lambda s}+L_{m} \bar{i}_{s \lambda s}\right),
$$

donde $\omega$, es la velocidad del marco de referencia y $\omega_{r}$ es la velocidad del rotor.

Reemplazando la ecuación de la corriente $\bar{i}_{s \lambda s}$ (4.65) en la expresión anterior (4.74), el fasor de la tensión rotórica en el marco de referencia orientado por el flujo estatórico está dado por:

$$
\bar{u}_{r \lambda s}=R_{r} \bar{i}_{r \lambda s}+L_{r}^{\prime} \frac{d \bar{i}_{r \lambda s}}{d t}+\frac{L_{m}^{2}}{L_{s}} \frac{d\left|\bar{i}_{m s}\right|}{d t}+j \omega_{s l}\left[\frac{L_{m}^{2}}{L_{s}}\left|\bar{i}_{m s}\right|+L_{r}^{\prime} \bar{i}_{r \lambda s}\right]
$$

donde

$$
\omega_{s l}=\omega-\omega_{r} .
$$

La expresión (4.75) puede expresarse empleando los ejes directos y en cuadratura como:

$$
T_{r}^{\prime} \frac{d i_{r x}}{d t}+i_{r x}=\frac{u_{r x}}{R_{r}}+\omega_{s l} T_{r}^{\prime} i_{r y}-\left(T_{r}-T_{r}^{\prime}\right) \frac{d\left|\bar{i}_{m s}\right|}{d t},
$$

y

$$
T_{r}^{\prime} \frac{d i_{r y}}{d t}+i_{r y}=\frac{u_{r y}}{R_{r}}-\omega_{s l} T_{r}^{\prime} i_{r x}-\omega_{s l}\left(T_{r}-T_{r}^{\prime}\right)\left(\left|\bar{i}_{m s}\right|\right),
$$

$\operatorname{con} T_{r}=\frac{L_{r}}{R_{r}} \mathrm{y} T_{r}^{\prime}=\frac{L_{r}^{\prime}}{R_{r}}=\frac{L_{r}-L_{m}^{2}}{L_{s}}$.

El acoplamiento entre las componentes de los ejes $x$ e $y$ puede eliminarse considerando que la operación de la máquina se realiza a flujo estatórico constante, es decir $\left(\left|\bar{i}_{m s}\right|=c t e\right)$. Las tensiones de desacoplamiento que deben adicionarse a los controladores de las corriente $i_{r x}$ e $i_{r y}$ están dadas por:

$$
u_{d r x}=-\omega_{s l} L_{r}^{\prime} i_{r y}
$$

y

$$
u_{d r y}=\omega_{s l} L_{r}^{\prime} i_{r x}+\omega_{s l}\left(L_{r}-L_{r}^{\prime}\right)\left|\bar{i}_{m s}\right|
$$

\subsubsection{Control vectorial orientado según flujo estatórico}

En este marco de referencia, las corrientes rotóricas $\left(i_{r x}, i_{r y}\right)$ corresponden directamente a las dos componentes de las corrientes estatóricas $i_{s x}$ e $i_{s y}$ en el marco de referencia de flujo estatórico. Es decir, a partir de la expresión de la corriente $\left(i_{s y}\right)$ dada en $(4.61)$ se puede observar que la corriente en el estator en el eje $y$ es proporcional a la corriente en el rotor 
en el eje $y$. Desarrollando la ecuación para la corriente en el estator en el eje $x$, la cual está dada por:

$$
i_{s x}=\left(\left|\bar{i}_{m s}\right|-i_{r x}\right) \frac{L_{m}}{L_{s}} .
$$

En este caso se asume que la resistencia $R_{s}$ se puede despreciar en estado estacionario Qizhong et al. (2011), lo cual es válido en máquinas de potencias mayores a $10 \mathrm{~kW}$. Además, se asume que la frecuencia $\omega_{1}$ es constante, $\left|\bar{i}_{m s}\right|$ es constante en estado estacionario y la velocidad de la corriente magnetizante $\left(\omega=\frac{d \rho_{s}}{d t}\right)$ es también constante. En este sentido, el módulo del fasor de la tensión de estator puede escribirse como:

$$
\left|\bar{u}_{s}\right|=\omega_{1} L_{m}\left|\bar{i}_{m s}\right|
$$

El módulo de la corriente magnetizante está dado por:

$$
\left|\bar{i}_{m s}\right|=\frac{\left|\bar{u}_{s}\right|}{L_{m} \omega_{1}} \text {. }
$$

De esta forma, se puede reemplazar la ecuación anterior en (4.81) para obtener una nueva expresión para $i_{s x}$ de la siguiente forma:

$$
i_{s x}=\left(\frac{\left|\bar{u}_{s}\right|}{L_{m} \omega_{1}}-i_{r x}\right) \frac{L_{m}}{L_{s}} .
$$

Teniendo en cuenta que $u_{s x}=0, u_{s y}=\left|\bar{u}_{s}\right|$ y $\left|\bar{i}_{m s}\right|=\frac{u_{s y}}{L_{m} \omega_{1}}$ se puede llegar a similares conclusiones que para la corriente en el estator en el eje $y$. Con el objetivo de analizar el intercambio de potencia por parte del DFIG se escriben las ecuaciones de la potencia activa en el estator como:

$$
P_{s}=\frac{3}{2} \operatorname{Re}\left(\bar{u}_{s \lambda s} \bar{i}_{s \lambda s}^{*}\right)=\frac{3}{2}\left(u_{s x} i_{s x}+u_{s y} i_{s y}\right),
$$

y la potencia reactiva en el estator es:

$$
Q_{s}=\frac{3}{2} \operatorname{Im}\left(\bar{u}_{s \lambda s} \bar{i}_{s \lambda s}^{*}\right)=\frac{3}{2}\left(u_{s y} i_{s x}-u_{s x} i_{s y}\right) .
$$

Aplicando las consideraciones previas podemos escribir la potencia activa y reactiva desarrollada en la máquina como:

$$
P_{s}=\frac{3}{2}\left(u_{s y} i_{s y}\right)=\frac{3}{2}\left|\bar{u}_{s}\right| i_{s y},
$$

y

$$
Q_{s}=\frac{3}{2}\left(u_{s y} i_{s x}\right)=\frac{3}{2}\left|\bar{u}_{s}\right| i_{s x} .
$$

De las expresiones de potencia activa y reactiva (4.87) y (4.88) en el marco de referencia según el flujo estatórico podemos observar que la inyección de potencia activa y reactiva puede ser controlado mediante las corrientes de estator en el eje $x$ e $y$ respectivamente.

En la Figura 4.6 se muestra el esquema de una estrategia de control para un DFIG en el cual se monitorea las corrientes del estator y del rotor, la velocidad angular del eje del rotor y las potencias activa y reactiva. En la figura se puede observar que el método requiere que se mida o que se estime la velocidad del rotor $\omega_{r}$. El valor del ángulo $\theta_{r}$ es obtenido integrando 


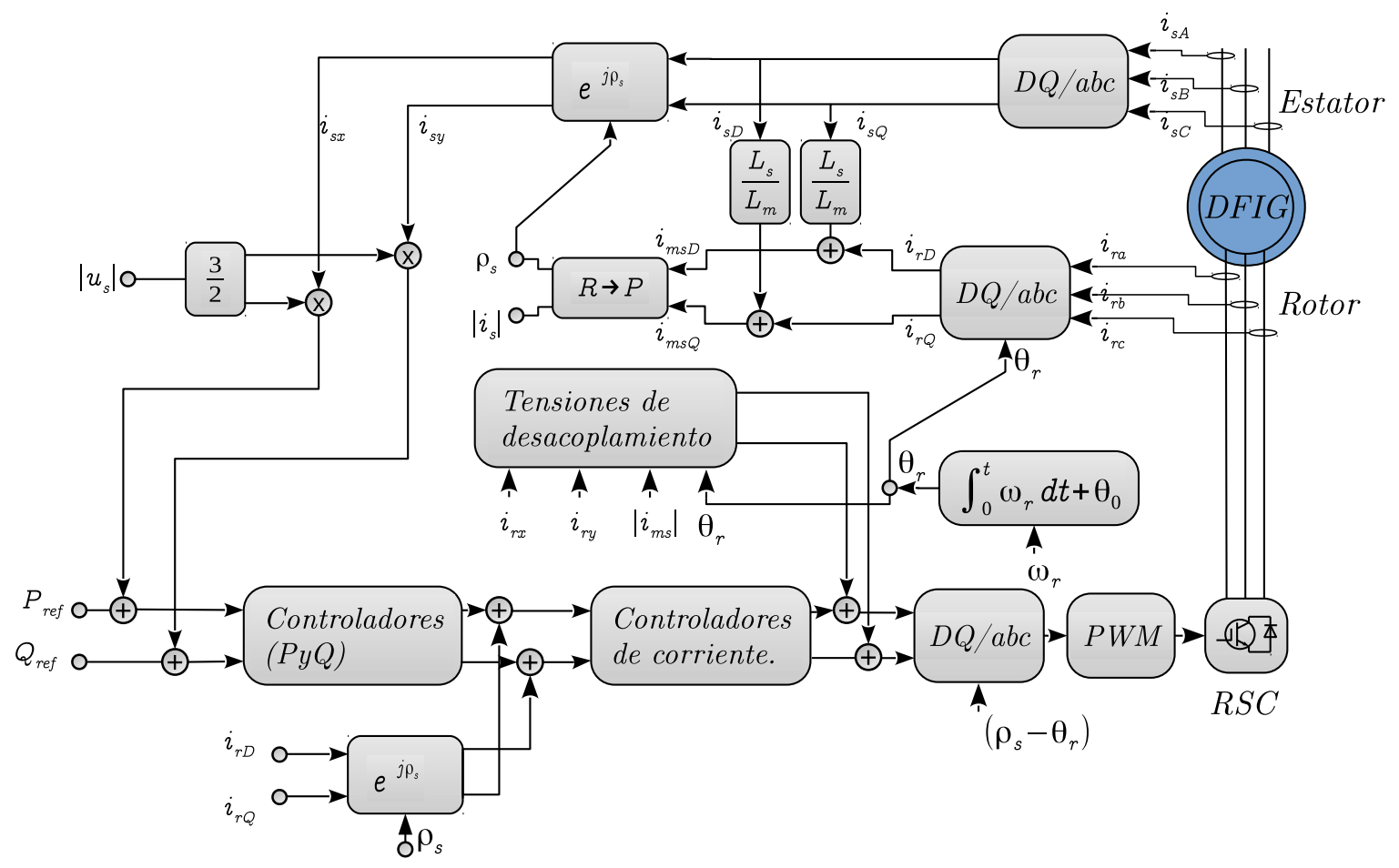

Figura 4.6: Esquema del control de una máquina de rotor bobinado (DFIG) empleando el marco de referencia según el flujo estatórico.

el valor de $\omega_{r}$, donde se considera que $\theta_{0}$ es el valor inicial del ángulo del rotor. Para el cálculo de la potencia activa y reactiva de la máquina se mide el módulo de la tensión de estator $\left|u_{s}\right|$ y la corriente del estator. La corriente del estator es transformada empleando el ángulo $\rho_{s}$. El ángulo de la corriente magnetizante en el estator $\left(\rho_{S}\right)$ es obtenido de las componentes del eje directo $i_{m s D}$ y en cuadratura $i_{m s Q}$ de la corriente estatórica magnetizante, expresada en el marco de referencia estacionario, convirtiendo de coordenadas rectangulares a polares, como se muestra en el esquema de la Figura 4.6. En la parte inferior derecha del esquema se puede observar el RSC el cual se encuentra conectado a los bornes del rotor y las señales de control correspondientes. La señal de control para al módulo PWM se obtiene de un lazo de control de corriente al cual se adicionan las tensiones de desacoplamiento dadas en las expresiones (4.79) y (4.80). Los valores de referencia para el lazo de corriente son obtenidos de un lazo previo que tiene como referencias valores de potencia activa y reactiva. Estos valores de potencia son comparados con el valor de la potencia reactiva y activa medida en el marco de referencia según el flujo estatórico.

\subsubsection{Control vectorial según la tensión de línea o flujo de red}

La estrategia de control orientado según el flujo de red (GFO, por su sigla en inglés Grid-Flux Oriented) puede lograr, de la misma forma que el marco de referencia de flujo estatórico, desacoplar la potencia activa y reactiva de un generador asincrónico de rotor bobinado (DFIG). El marco de referencia de flujo estatórico ante una condición de tensión de la red distorsionada necesita la estimación precisa del fasor de flujo del estator en cada instante (Petersson et al., 2004). 
El esquema de la implementación de la estrategia de control del sistema según el flujo de red se muestra en la Figura 4.7. El método asume que la dirección positiva del eje D coincide con la dirección del fasor de la tensión estatórica y el eje Q está a $\pi / 2$. Además, el sistema que implementa la estrategia de control orientado al vector de tensión del estator está equipado con un lazo de seguimiento de fase PLL con el objetivo de seguir el ángulo y la frecuencia de la tensión de fase en el estator (Petersson et al., 2004).

De acuerdo a Petersson et al. (2004) se propone un flujo virtual como:

$$
\bar{\lambda}_{g}=\frac{\bar{u}_{s}}{j \omega_{g}}=-\frac{j\left|u_{s}\right| e^{j \theta_{g}}}{\omega_{g}},
$$

donde $\omega_{g}$ es la frecuencia de la tensión de la red eléctrica, y $\theta_{g}$ es la fase de la tensión respecto del eje de referencia. A partir de $\omega_{g}$ el flujo virtual es vinculado a la tensión de la red eléctrica. Este nuevo marco de referencia no implica ninguna consideración extra de las que se ya se han desarrollado. Si se desprecia la resistencia del estator es posible escribir la ecuación de tensión en el estator en el nuevo marco de referencia como:

$$
\bar{u}_{s g}=R_{s} \bar{i}_{s g}+\frac{d \bar{\lambda}_{s}}{d t}+j \omega_{1} \bar{\lambda}_{s} \approx j \omega_{1} \bar{\lambda}_{s},
$$

donde $\omega_{1}=\omega_{g}$.

La relación entre $\theta_{1}$ (ángulo sincrónico) y $\theta_{g}$ para un marco de referencia orientado a la tensión de estator es:

$$
\theta_{1}=\theta_{g}-\frac{\pi}{2}
$$

Un esquema detallado del control de un generador de rotor bobinado que emplea control vectorial con flujo orientado de red es mostrado en la Figura 4.7. A diferencia del esquema por flujo estatórico se puede observar en la Figura 4.7 la adición de un PLL que permite obtener el módulo de la tensión del estator como así también el ángulo de fase de la tensión.

Las ecuaciones para la potencia activa y reactiva en un (DFIG) empleando este nuevo marco de referencia son:

$$
P_{s}=\frac{3}{2}\left|\bar{u}_{s}\right| i_{s y},
$$

y

$$
Q_{s}=\frac{3}{2}\left|\bar{u}_{s}\right| i_{s x} .
$$

Estas expresiones son válidas para controlar la inyección de potencia, para todo el rango de funcionamiento de un DFIG a diferencia del control vectorial de campo, en el cual las expresiones (4.87) y (4.88) son válidas únicamente en estado estacionario (Mohseni et al., 2011).

Además, utilizando el marco de referencia orientado según el flujo de red la estabilidad y el amortiguamiento del sistema es independiente de las corrientes del rotor en contraste con el marco de referencia según el flujo estatórico. Esto implica que es posible magnetizar la máquina completamente a través del rotor sin reducir el amortiguamiento del sistema. Por otra parte con las actuales regulaciones acerca de capacidad para manejar una falla, este marco de referencia puede inyectar más potencia reactiva en el punto de conexión beneficiando la estabilidad de la red eléctrica (Petersson et al., 2004). 


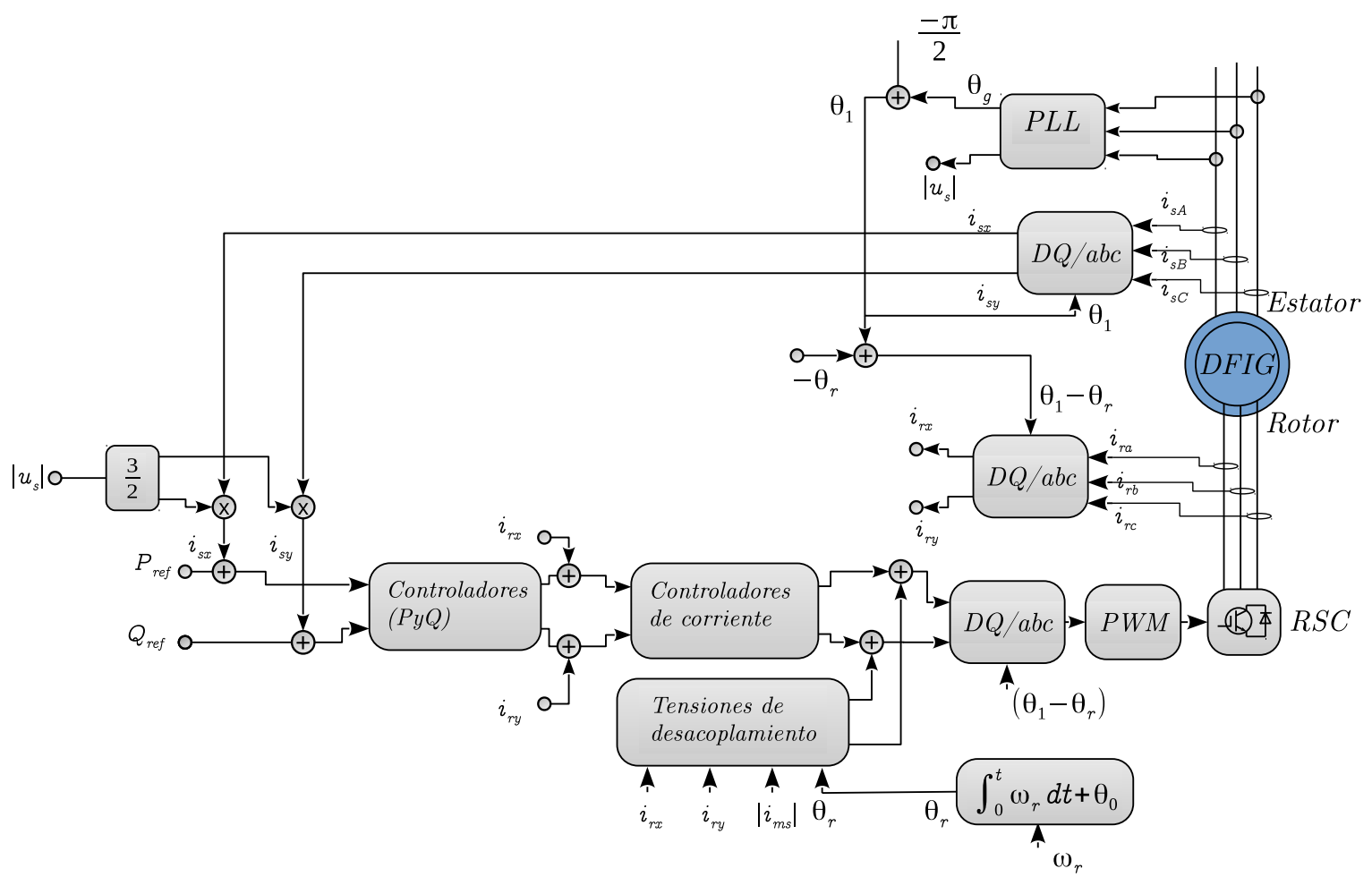

Figura 4.7: Esquema del control de una máquina de rotor bobinado (DFIG) empleando el marco de referencia orientado por flujo de red.

\subsection{Retrospección}

En este capítulo se ha realizado un repaso y análisis del control vectorial en general y del control vectorial orientado según el flujo estatórico. Se han obtenido distintas expresiones para el par electromagnético según el marco de referencia elegido. En particular se puede decir que el control de la máquina de inducción empleando el marco de referencia por flujo estatórico tiene como virtud, en estado estacionario, el desacoplamiento del control de la potencia activa y reactiva del mismo. También se abordó el análisis del marco de referencia orientado según el marco de referencia por flujo de red. Desde un punto de vista referido a la estabilidad del sistema, se ha demostrado en la literatura que el marco de referencia orientado según la tensión de red puede aportar más potencia reactiva que el marco de referencia de flujo orientado. Pensando que un generador de rotor bobinado debe estar preparado para cumplir con las reglamentaciones de red frente a una falla como un hueco de tensión este debería ser capaz de aportar grandes cantidades de potencia reactiva para tratar de restablecer la tensión en el punto de conexión del generador. En este sentido, la estrategia de control empleando el marco de referencia de flujo de red permite la utilización de las expresiones de potencia activa y reactiva son válidas en todo el rango de funcionamiento.

Las herramientas presentadas en este capítulo permiten el análisis de un aerogenerador equipado con un generador de rotor bobinado. El aerogenerador puede vincularse a una red eléctrica para contribuir con la inyección de potencia con capacidades de manejar una falla. 


\section{Capítulo 5}

\section{Control Basado en Pasividad}

\section{Resumen}

En este capítulo se presentan los conceptos básicos de la teoría de Pasividad. Se muestran las propiedades de los sistemas pasivos. Se describe la estabilidad de un sistema en el sentido de Lyapunov. Se presenta la modelización de un sistema empleando conceptos de energía y la modelización usando puertos. En particular se presentan las técnicas de moldeo de energía y de moldeo de potencia las cuales brindan mayor flexibilidad en la obtención de la función de Lyapunov.

También se presenta la adición de un control integral a la estructura basada en las ecuaciones de Brayton-Moser.

\subsection{Introducción}

En los últimos 20 años ha habido un gran desarrollo en técnicas de control no lineales (Kokotovic, 2004; Haddad y VijaySekhar, 2008). Recientemente, se han explorado técnicas de control que aprovechan la estructura del sistema por sobre las técnicas más tradicionales que tratan de imponer un comportamiento dinámico predeterminado a través de la cancelación de las no linealidades del sistema (Van der Schaft y Jeltsema, 2014).

En particular, el control basado en pasividad (CBP) ha surgido como un método de diseño de control no lineal basado en un modelo del sistema que explota las propiedades físicas del mismo.

La energía es un concepto fundamental y existen diferentes motivaciones para modelar un sistema físico utilizando la energía. En principio se puede decir que la energía es un concepto 
común entre diferentes especialidades de las ciencias, lo cual puede facilitar la comunicación en un trabajo interdisciplinario.

Las ideas del control basado en pasividad emplean conceptos básicos de energía. De esta manera, se aportan herramientas de análisis y control que brindan una intuición física de la acción de control. Por otro lado, un sistema físico puede ser estudiado como un subsistema que intercambia energía con el medio y es común que el sistema dinámico pueda ser visto como un dispositivo que transforma la energía. La técnica de control basada en pasividad que aprovecha esta característica es denominada moldeo de energía, la cual balancea o da forma a la función de energía del sistema de lazo cerrado. Los conceptos de la técnica de control mediante balance de energía tienen sus orígenes en trabajos acerca de manipuladores robóticos en la década del 80 (Ortega y Spong, 1989). Además, la acción de control implementada en un controlador puede ser entendida en términos de energía, donde el controlador mediante el intercambio de la misma modifica las características dinámicas del sistema controlado (Ortega et al., 2001a). Es decir, el controlador es interpretado como un dispositivo que intercambia energía con la planta y es diseñado de forma tal que el sistema dinámico de lazo cerrado presente las características deseadas. Además, la interconexión de sistemas de diferentes dominios (mecánicos, eléctricos, etc) puede ser considerada añadiendo las contribuciones de cada uno individualmente. El control por pasividad ha sido extendido a diferentes aplicaciones como sistemas mecánicos, electromecánicos, convertidores electrónicos, etc, (Ortega, 1998).

Por otra parte, la teoría de sistemas hamiltonianos con puertos está basada en conceptos de mecánica clásica lo cual muchas veces permite la sintonización de controladores aprovechando la estructura física del sistema y permite tratar de forma unificada sistemas que provienen de diferentes dominios físicos (Batlle y Dòria-Cerezo, 2010).

Una formulación de control basado en pasividad conocida como "interconexión y amortiguamiento" fue introducida por Ortega y coautores en (Ortega et al., 2001a). En este contexto, la gestión del control debe ser interpretada en términos de acciones dirigidas a la construcción de una función de energía que modifique la energía del sistema no controlado para dar garantías de estabilidad y correspondencia con el comportamiento deseado.

Además del enfoque de balance de energía se ha propuesto un formalismo que moldea la potencia de un sistema (Ortega et al., 2003a). El control por moldeo de potencia basado en pasividad, tiene sus orígenes en el estudio de circuitos eléctricos (Brayton y Moser, 1964a,b). Los resultados obtenidos por esta técnica de control son de interés para su aplicación en sistemas de control y resulta una alternativa al método de moldeo de energía (Jeltsema y Scherpen, 2007). La técnica de control por moldeo de potencia es la que se eligió para plantear los problemas a resolver a lo largo de la tesis.

\subsection{Conceptos previos}

En esta sección se presentan las propiedades y los conceptos básicos relacionados a los sistemas pasivos. Para una interpretación más clara también se resumen conceptos de la estabilidad en el sentido de Lyapunov. Por último, la estabilidad en los sistemas pasivos es presentada con el objeto de su posterior utilización. 


\subsubsection{Disipatividad y pasividad}

Tanto los sistemas disipativos como los pasivos son una clase de sistemas en los cuales el intercambio de energía con el medio ambiente juega un rol importante. Pasividad es la propiedad tal que la tasa de incremento del almacenamiento es no mayor que la tasa suministrada. En otras palabras un sistema pasivo no puede almacenar más energía que la que es suministrada por la fuente externa siendo la diferencia la energía disipada en el mismo. Cualquier incremento en el almacenamiento de energía en un sistema pasivo es debido a un aporte de una fuente externa. De esta manera, la interpretación de la pasividad del sistema a partir de los conceptos de energía esta íntimamente relacionada con la física del sistema y en particular con su estabilidad.

\section{Disipatividad}

A principios de 1970, se sistematizaron los conceptos de disipatividad respecto a las nociones de funciones de almacenamiento $H(x)$ y a la tasa de suministro $w(u, y)$, donde $x \in \mathbb{R}^{n}$ son los estados del sistema, $u \in \mathbb{R}^{m}$ es la entrada e $y \in \mathbb{R}^{m}$ la salida (Willems, 1972). La función de almacenamiento mide la cantidad de energía que es almacenada en el sistema, mientras que la función de tasa de suministro es utilizada para analizar la energía que fluye en el sistema.

Sea un sistema dinámico $\sum$ modelado a través de ecuaciones diferenciales ordinarias con un vector de entrada $u$ y un vector de salida $y$, dado por

$$
\sum:\left\{\begin{array}{l}
\dot{x}=f(x, u) \\
y=h(x, u)
\end{array},\right.
$$

y $x(0)=x_{0} \in \mathbb{R}^{n}$. La ecuación (5.1) define un operador dinámico causal como $\sum: \mathcal{L}_{2 e} \rightarrow$ $\mathcal{L}_{2 e}: u \rightarrow y^{1}$ con una función de suministro $w(u, y): \mathbb{R}^{m} \times \mathbb{R}^{m} \rightarrow \mathbb{R}$, la cual es localmente integrable, es decir

$$
\int_{t_{0}}^{t_{1}}|w(u(t), y(t))| d t \leq \infty \forall t_{0} \leq t_{1}
$$

y sea $\mathcal{X}$ un subconjunto $\mathbb{R}^{n}$ que contiene el origen. Entonces, podemos decir que el sistema $\sum$ es disipativo en $\mathcal{X}$ con tasa de suministro $w(u, y)$ si existe una función de almacenamiento de energía diferenciable $(H(x))$ tal que $H(x) \geq 0$ y $H(0)=0$

$$
H\left(x\left(t_{1}\right)\right)-H(x(0)) \leq \int_{0}^{t_{1}} w(u(t), y(t)) d t
$$

para todo $u$ y todo $t_{1} \geq 0$ tal que $x(t) \in \mathcal{X}$ para todo $t \in\left[0, t_{1}\right]$.

La desigualdad (5.2) es llamada desigualdad de disipación y expresa que la energía en un tiempo futuro $t_{1}$ menos la energía almacenada en el tiempo $t=0$ es menor o igual a la energía suministrada en el intervalo de tiempo $\left[0, t_{1}\right]$.

\footnotetext{
${ }^{1}$ Donde, la definición del espacio $\mathcal{L}_{2 e}$ es llevada a cabo considerando el conjunto $\Xi$ de todas las funciones del tiempo con valores reales medibles, $f(t): \mathbb{R}^{+} \rightarrow \mathbb{R}^{n}$, (donde $\mathbb{R}^{+}=[0, \infty)$ ). El espacio extendido $\mathcal{L}_{2 e}$ es definido como

$$
\mathcal{L}_{2 e} \triangleq\left\{x \in \Xi \mid\|f\|_{2 T}^{2} \triangleq \int_{0}^{t_{1}}\|f\|^{2} d t<\infty \forall t_{1}\right\}
$$

con la norma estándar euclídea $\|$.$\| . Este conjunto forma un espacio vectorial normado sobre el campo de los$ números reales con norma 2 (Ortega, 1998).
} 


\section{Pasividad}

Pasividad es un caso especial en el marco de disipatividad, en el cual dado un sistema $\sum$, expresado como (5.1), es pasivo si éste es disipativo con tasa de suministro $w(u, y)=u^{T} y$ (Ortega, 1998; Kokotovic y Arcak, 2001). Es decir, si la función de almacenamiento es diferenciable, se puede escribir:

$$
\dot{H}(x(t)) \leq u^{T} y,
$$

donde $u^{T} y$, generalmente es interpretado como la potencia suministrada al sistema (Van der Schaft y Jeltsema, 2014).

La forma integral de la desigualdad anterior puede escribirse como

$$
H\left(x\left(t_{1}\right)\right)-H(x(0)) \leq \int_{0}^{t_{1}} u^{T} y d t .
$$

La expresión anterior muestra que un sistema pasivo no puede almacenar más energía que la suministrada por la fuente externa $\left(\int_{0}^{t_{1}} u^{T} y d t\right)$ por lo tanto la diferencia entre dichas energías es la energía disipada por el sistema. Por otra parte, el sistema es llamado sin pérdidas si cumple con la igualdad de la expresión (5.3).

Se dice que un sistema $\sum$ es estrictamente pasivo con respecto a la entrada si es disipativo con tasa de suministro

$$
w(u, y)=u^{T} y-\delta_{i}\|u\|^{2},
$$

donde $\delta_{i}>0$. Además, $\sum$ es estrictamente pasivo con respecto a la salida, si es disipativo con tasa de suministro de energía

$$
w(u, y)=u^{T} y-\delta_{0}\|y\|^{2},
$$

$\operatorname{con} \delta_{0}>0$ (Ortega, 1998).

\subsubsection{Interconexión de sistemas pasivos}

Los métodos de diseño basados en pasividad suelen explotar la estructura del sistema a partir de la interpretación de la interconexión de subsistemas. Existen dos interconexiones básicas que son la interconexión en paralelo y por realimentación. Como se demuestra a continuación la interconexión de los sistemas $S_{1}$ y $S_{2}$ en paralelo o por realimentación es pasiva.

\section{Interconexión en paralelo}

En la Figura 5.1 se pueden observar dos sistemas $S_{1}$ y $S_{2}$ pasivos o estrictamente pasivos con respecto a la salida, conectados en paralelo con entrada común $u$ y salidas $y_{1}$ e $y_{2}$. Se asume que los sistemas $S_{1}$ y $S_{2}$ están expresados en la forma de (5.1).

Esta interconexión constituye un nuevo sistema dado por

$$
\sum_{1}=\left\{\begin{array}{rl}
\dot{x_{1}} & =f_{1}\left(x_{1}, u\right) \\
\dot{x_{2}} & =f_{2}\left(x_{2}, u\right) \\
y & =h_{1}\left(x_{1}, u\right)+h_{2}\left(x_{2}, u\right)
\end{array},\right.
$$


donde la última expresión que representa el sistema en paralelo está bien definida en el sentido que existe una solución para las ecuaciones diferenciales ordinarias.

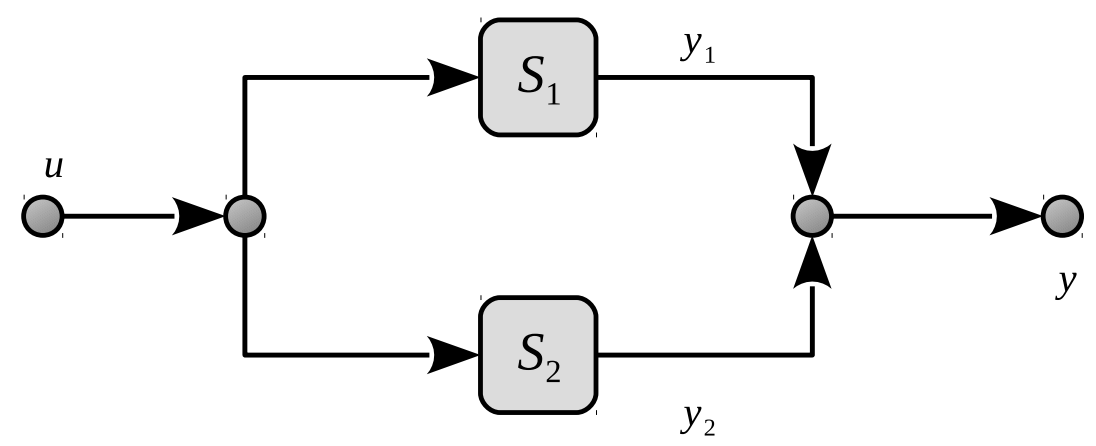

Figura 5.1: Interconexión en paralelo de sistemas pasivos.

La propiedad de pasividad de la conexión en paralelo se puede demostrar a partir de las funciones de almacenamiento $H_{1}\left(x_{1}\right)$ y $H_{2}\left(x_{2}\right)$ de los respectivos sistemas $S_{1}$ y $S_{2}$ de forma tal que

$$
H_{i}\left(x_{i}\left(t_{1}\right)\right)-H_{i}\left(x_{i}(0)\right) \leqslant \int_{0}^{t_{1}} u_{i}^{T} y_{i} d t, i=1,2
$$

con los estados $x$ definido como $x=\left(x_{1}, x_{2}\right)^{T}$. Sumando la expresión (5.8) con $i=1$ y 2 y definiendo como función de almacenamiento del sistema completo a $H(x)=H_{1}\left(x_{1}\right)+H_{2}\left(x_{2}\right)$ (siendo $H(x)$ semidefinido positivo)

$$
H\left(x\left(t_{1}\right)\right)-H(x(0)) \leqslant \int_{0}^{t_{1}}\left(u^{T} y_{1}+u^{T} y_{2}\right) d t=\int_{0}^{t_{1}} u^{T} y d t .
$$

Esta última expresión es igual a la definición de pasividad dada previamente considerando que la tasa de suministro es $w(u, y)=u^{T} y$ con $y=y_{1}+y_{2}$. De esta forma, se demuestra que la interconexión en paralelo de dos sistemas pasivos es pasiva.

\section{Interconexión en realimentación}

La interconexión en realimentación se presenta en la Figura 5.2. De dicho esquema es posible escribir las entradas de los sistemas $S_{2}$ y $S_{1}$ como $u_{2}=y_{1}$ y $u_{1}=r-y_{2}$, respectivamente.

El nuevo sistema queda representado como

$$
\sum_{2}=\left\{\begin{aligned}
\dot{x_{1}} & =f_{1}\left(x_{1},-h_{2}\left(x_{2}, h_{1}\left(x_{1}\right)\right)+r\right) \\
\dot{x_{2}} & =f_{2}\left(x_{2}, h\left(x_{1}\right)\right) \\
y & =h\left(x_{1}\right)
\end{aligned}\right.
$$

De la misma forma que en el caso anterior, es decir, sumando las expresiones $H_{1}(x) \mathrm{y}$ $H_{2}(x)$ para diferentes índices $i$ entonces $x=\left(x_{1}, x_{2}\right)^{T}$ y $H(x)=H_{1}(x)+H_{2}(x)$, resulta

$$
H\left(x\left(t_{1}\right)\right)-H(x(0)) \leqslant \int_{0}^{t_{1}}\left(u_{1}^{T} y_{1}+u_{2}^{T} y_{2}\right) d t .
$$




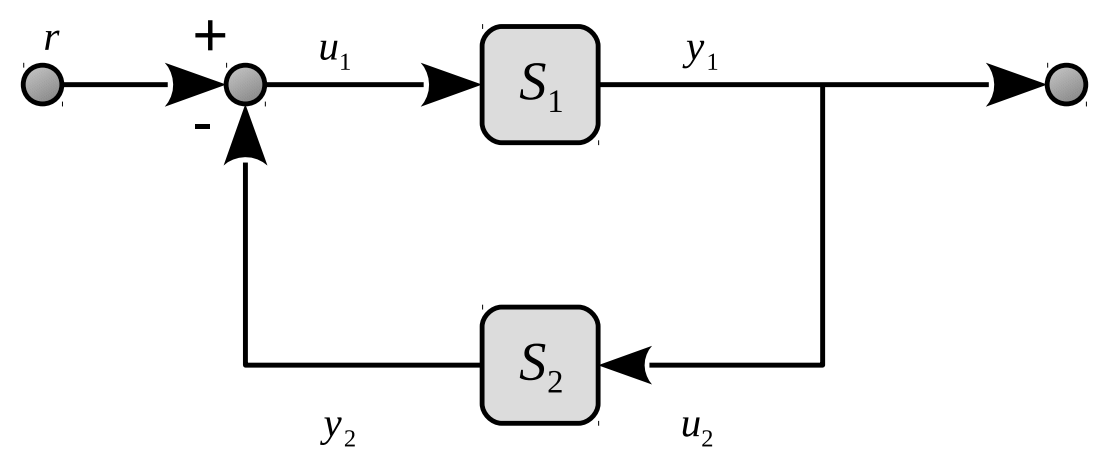

Figura 5.2: Interconexión en realimentación de sistemas pasivos.

Reemplazando en la ecuación anterior por las entradas $u_{1}=r-y_{2}$ y $u_{2}=y_{1}$ se obtiene

$$
H\left(x\left(t_{1}\right)\right)-H(x(0)) \leqslant \int_{0}^{t_{1}} r^{T} y_{1} d t
$$

lo cual demuestra que la interconexión de dos sistemas pasivos por realimentación es pasiva.

\section{Pre y post multiplicación por una matriz}

A menudo en la interconexión de sistemas suele ocurrir una transformación de la entrada y de la salida de un sistema pasivo $S(x)$ mediante una matriz $M(x)$ dependiente de los estados del mismo. Este tipo de transformación se observa en la Figura 5.3, siendo de interés saber si se retienen las propiedades de pasividad del sistema $S(x)$.

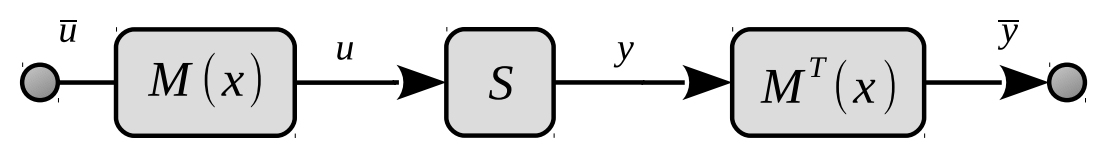

Figura 5.3: Pre y post multiplicación por una matriz dependiente de los estados.

Observando la Figura 5.3 es posible escribir la entrada para el sistema $S$ como $u=M(x) \bar{u}$. La salida luego de la pre y post multiplicación por la matriz $M(x)$ es $\bar{y}=M^{T}(x) y$. Si $S$ es pasivo con una función de almacenamiento $H(x)$

$$
H\left(x\left(t_{1}\right)\right)-H(x(0)) \leqslant \int_{0}^{t_{1}} u^{T} y d t
$$

entonces el sistema transformado es también pasivo con la misma función de almacenamiento, ya que:

$$
H\left(x\left(t_{1}\right)\right)-H(x(0)) \leqslant \int_{0}^{t_{1}} u^{T} y d t=\int_{0}^{t_{1}} \bar{u}^{T} M^{T}(x) y d t=\int_{0}^{t_{1}} \bar{u}^{T} \bar{y} d t .
$$

De esta forma la propiedad de pasividad del sistema afectado por la matriz $M(x)$ se mantiene.

Además, puede demostrarse que el sistema que resulta de la interconexión de los sistemas pasivos $S_{1}$ y $S_{2}$ retiene las propiedades de pasividad si uno o ambos son pre multiplicados por $M\left(x_{1}, x_{2}\right)$ y post multiplicados por una matriz $M^{T}\left(x_{1}, x_{2}\right)$ que depende de los estados $x=\left(x_{1}, x_{2}\right)^{T}$. 


\subsubsection{Exceso y falta de pasividad}

Frente a la interconexión de dos sistemas uno de los cuales es pasivo y el otro no, resulta de interés conocer si la misma es pasiva. En este sentido, se comentan las siguientes definiciones con respecto al exceso y la falta de pasividad en un sistema interconectado.

Se dice que

- un sistema en realimentación en la salida es pasivo, (OFP, por sus siglas en inglés Output Feedback Passive) si este es disipativo con respecto a $w(u, y)=u^{T} y-\rho y^{T} y$ para algún $\rho \in \mathbb{R}^{+}$.

- un sistema es feedforward de entrada pasiva, (IFP, por sus siglas en inglés Input Feedforward Passive) si este es disipativo con respecto a $w(u, y)=u^{T} y-\nu u^{T} u$ para algún $\nu \in \mathbb{R}$.

De esta forma se puede cuantificar el exceso o la falta de pasividad con la notación $O F P(\nu)$ y $\operatorname{IFP}(\rho)$. De acuerdo a la convención desarrollada, un signo positivo para $\rho$ y $\nu$ significa que el sistema tiene un exceso de pasividad y viceversa.

Otro concepto análogo a IFP $(\nu>0)$ en pasividad es el concepto de sistemas estrictamente pasivos el cual requiere que

$$
\int_{0}^{t_{1}} u^{T} y d t \geqslant \nu \int_{0}^{t_{1}} u^{T} u d t+\beta
$$

para algún $\nu>0$ y $\beta \in \mathbb{R}$.

Por ejemplo, considerando un sistema simple $y=-k u$, donde $k>0$. Este sistema es estático y su espacio de estados es nulo, además, la única función de almacenamiento es $H=0$, con $u y=-k u^{2}$. Aplicando la definición de la expresión (5.4), la integral es negativa, lo cual viola la definición de pasividad.

Evaluando un sistema pasivo $S$ con una interconexión en paralelo con $-k I$, para un caso multivariable, donde $I$ es la matriz identidad de $m \times m$ y $k>0$. Para que esta interconexión sea pasiva su tasa de suministro $u^{T} \bar{y}$ tiene que satisfacer la expresión (5.4). Siendo $\bar{y}=y-k u$, es decir

$$
u^{T} y=u^{T} \bar{y}+k u^{T} u .
$$

A su vez por la definición de la interconexión en paralelo de $S$ con $-k I$, el nuevo sistema es pasivo si $S$ es disipativo con $\nu \geq k$ y respecto a la tasa de suministro

$$
w(u, y)=u^{T} y-\nu u^{T} u,
$$

si verifica

$$
H\left(x\left(t_{1}\right)\right)-H(x(0)) \leq \int_{0}^{t_{1}} u^{T} \bar{y}-(\nu-k) u^{T} u d t .
$$

En el caso de la interconexión en realimentación de $S$ con $-k I$. La entrada al sistema $S$ es $u=r+k y$. El sistema interconectado es pasivo con una función de almacenamiento $H(x)$, con respecto a la tasa de suministro

$$
w(u, y)=u^{T} y-\rho y^{T} y,
$$


si

$$
H\left(x\left(t_{1}\right)\right)-H(x(0)) \leq \int_{0}^{t_{1}} y^{T} r-(\rho-k) y^{T} y d t \leq \int_{0}^{t_{1}} y^{T} r d t .
$$

Con $\rho \geq k$, se cumple que la conexión es pasiva. En cada uno de los dos casos la falta de pasividad de $-k I$ es compensada por el exceso de pasividad de $S$. Este exceso de pasividad garantiza la pasividad del sistema interconectado.

\subsubsection{Estabilidad en el sentido de Lyapunov}

Los resultados obtenidos por Lyapunov presentan un marco de trabajo fuerte para analizar la estabilidad de sistemas dinámicos no lineales así como el diseño de controladores que garanticen la estabilidad del lazo cerrado. En esta subsección se presenta brevemente la estabilidad según Lyapunov en base a Sepulchre et al. (1997).

\section{Tipos de estabilidad}

Considere el siguiente sistema dado por

$$
\dot{x}=f(x),
$$

donde $x \in \mathbb{R}^{n}$ y $f: \mathbb{R}^{n} \rightarrow \mathbb{R}^{n}$ es localmente Lipschitz continua y sea $x=x^{*}$ un punto de equilibrio, es decir las raíces de la ecuación (5.19) que describe el sistema. Específicamente, un punto de equilibrio se puede decir que es estable si para una pequeña perturbación el sistema permanece en las cercanías del punto de equilibrio.

Una de las propiedades de los sistemas como (5.19) es que existe una única solución y puede ser expresada como $x\left(t ; x_{0}, t_{0}\right)$.

La solución del sistema (5.19) es invariante ante un desplazamiento en el tiempo. Es decir, $x\left(t+T ; x, t_{0}+T\right)=x\left(t ; x_{0}, t\right)$.

Las propiedades de estabilidad son uniformes y no dependen de $t_{0}$, por lo tanto, sin pérdida de generalidad asumimos que $t_{0}=0$. De esta forma podemos escribir la solución como $x\left(t ; x_{0}\right)$ en vez de $x\left(t ; x_{0}, 0\right)$.

La estabilidad de Lyapunov es una propiedad continua de $x\left(t ; x_{0}\right)$ con respecto a $x_{0}$. Si el estado inicial $x_{0}$ es perturbado a $\tilde{x}_{0}$, entonces, la solución del sistema perturbado $x\left(t ; \tilde{x}_{0}\right)$ es requerida para estar próximas a $x\left(t ; x_{0}\right) \forall t \geq 0$. Además, el error entre las soluciones $x\left(t ; \tilde{x}_{0}\right)$ y $x\left(t ; x_{0}\right)$ debe converger a cero para $t \rightarrow \infty$ para estabilidad asintótica. Luego la solución es de $x\left(t ; x_{0}\right)$, que permiten definir la estabilidad del equilibrio de (5.19), pueden ser:

- Acotada, si existe una constante $K\left(x_{0}\right)$ tal que

$$
\left\|x\left(t ; x_{0}\right)\right\| \leq K\left(x_{0}\right), \forall t \geq 0 .
$$

- Estable, si para cada $\epsilon>0 \exists \delta(\epsilon)>0$ tal que

$$
\left\|\tilde{x}_{0}-x_{0}\right\|<\delta \Rightarrow\left\|x\left(t ; \tilde{x}_{0}\right)-x\left(t ; x_{0}\right)\right\|<\epsilon, \forall t \geq 0 .
$$


- Atractivo, si $\exists r\left(x_{0}\right)>0$ tal que

$$
\left\|\tilde{x}_{0}-x_{0}\right\|<r\left(x_{0}\right) \Rightarrow \lim _{t \rightarrow \infty}\left\|x\left(t ; \tilde{x}_{0}\right)-x\left(t ; x_{0}\right)\right\|=0 .
$$

- Asintóticamente estable, si es estable y atractivo.

- Inestable, si el sistema no es estable.

Se define un punto de equilibrio $\left(x^{*}\right)$ como globalmente estable (GE), si $x^{*}$ es estable y si todas las soluciones del sistema (5.19) son acotadas.

El punto de equilibrio $x^{*}$ es globalmente asintóticamente estable (GAE), si es asintóticamente estable y su región de atracción es $\mathbb{R}^{n}$.

\section{Estabilidad}

Sea $x=0$ un punto de equilibrio de un sistema dinámico dado por (5.19), una función $f(x)$ Lipschitz continua y además sea una función $V(x): \mathbb{R}^{n} \rightarrow \mathbb{R}^{+}$, continua y diferenciable (donde, $\mathbb{R}^{+}=[0, \infty)$ ) y radialmente no acotada de forma tal que

$$
\dot{V}=\frac{\partial V}{\partial x}(x) f(x) \leq 0, \forall x \in \mathbb{R}^{n}
$$

Entonces si el punto de equilibrio $(x=0)$ es globalmente estable (GE) y todas las soluciones de la expresión (5.19) convergen al conjunto donde $\dot{V}(x)=0$ y si además $\dot{V}$ es definida negativa, entonces $x=0$ es globalmente asintóticamente estable (GAE). Esta función $V(x)$ se la conoce como función de Lyapunov.

Las funciones de Lyapunov no son únicas y la tarea de construirlas no es fácil. Esto es debido a que la teoría de Lyapunov no es un método constructivo.

En general, las funciones de Lyapunov se han aplicado con el objetivo de estudiar la estabilidad de un sistema. El problema de control consiste en obtener una ley de control para el sistema a lazo cerrado

$$
\dot{x}=f(x, u(x)),
$$

de forma tal que posee un punto de funcionamiento estable en el origen, es decir, se puede buscar una acción de control $u(x)$ que logre que $\dot{V}(x, u(x))$ sea definida negativa.

La relación entre Pasividad y la estabilidad de Lyapunov puede ser establecida empleando la función de almacenamiento como función de Lyapunov.

\section{Principio de invariancia de Barbashin-Krasovskii-LaSalle}

Sea un conjunto $\Omega \subset D$ compacto que es positivamente invariante ${ }^{2}$ con respecto al sistema dinámico $\dot{x}=f(x)$ y una función $V: \mathbb{R}^{n} \rightarrow \mathbb{R}$ definida positiva que es continuamente

\footnotetext{
${ }^{2}$ Un conjunto $H$ se dice positivamente invariante si:

$$
x(0) \epsilon H \Rightarrow x(t) \epsilon H, \forall t \geq 0 .
$$
}


diferenciable tal que $\dot{V} \leq 0$ en $\Omega$. Se define un conjunto $E \subset \Omega$ donde se cumple que $\dot{V}=0$ y además sea $M$ el mayor conjunto invariante en $E$. Entonces, cada solución que comienza en $\Omega$ converge a $M$ cuando $t \rightarrow \infty$ (Sastry, 1999; Sepulchre et al., 1997). Una aplicación del principio de invariancia es la siguiente condición asintótica de estabilidad.

\section{Corolario estabilidad asintótica}

Sea $x=0$ el punto de equilibrio del sistema $\dot{x}=f(x), V(x)$ una función continuamente diferenciable definida de la misma forma que en el principio de invariancia y un conjunto $E=\left\{x \in \mathbb{R}^{n} \mid \dot{V}(x)=0\right\}$ suponiendo que ninguna solución puede permanecer en $E$ más que la trivial, entonces el origen es asintóticamente estable (Sastry, 1999; Sepulchre et al., 1997).

\subsubsection{Lema de Kalman-Yakubovich-Popov (KYP)}

Un resultado clave que conecta los conceptos de pasividad con la existencia de una función cuadrática lineal es el lema KYP, también conocido como lema real positivo (Sepulchre et al., 1997; Kokotovic y Arcak, 2001). El lema puede considerarse como una generalización de las funciones de Lyapunov en la teoría de estabilidad. El lema puede ser utilizado en las definiciones de margenes de estabilidad para sistemas no lineales. Además, involucra una relación entre una representación en el espacio de estados y una condición en el dominio de la frecuencia.

Considerando un sistema lineal e invariante en el tiempo (LTI), completamente controlable y observable estable con una representación mínima (Ortega, 1998) dado por

$$
\left\{\begin{array}{l}
\dot{x}=A x+B u \quad ; x(0)=x_{0} \\
y=C x
\end{array}\right.
$$

donde $x \in \mathbb{R}^{n}$ y $u, y \in \mathbb{R}^{m}$, la matriz de transferencia es $G(s)=C(s I-A)^{-1} B$ es real positiva y se satisfacen las siguientes condiciones equivalentes

- Todos los polos están sobre el semi-plano izquierdo y los autovalores de la matriz $A$ sobre el eje imaginario son simples y su Hermitiano es una matriz definida positiva (Ortega, 1998).

$$
G(j \omega)+G^{T}(-j \omega) \geq 0, \forall \omega \in \mathbb{R} .
$$

- El sistema (5.24) es pasivo si y solo si existe una matriz $P=P^{T}>0$ y $Q=Q^{T} \geq 0$ tal que

$$
\begin{gathered}
A^{T} P+P A=-Q \\
P B=C^{T}
\end{gathered}
$$

- El operador $G: u \mapsto y$ es pasivo con una función de almacenamiento $V(x)=\frac{1}{2} x^{T} P x$.

Como consecuencia, si $G(s)$ es real positiva, entonces para una realización mínima de $G(s)$, existe $P>0$ que satisface las condiciones de pasividad (Sepulchre et al., 1997). 


\subsubsection{Estabilidad de sistemas pasivos}

Para utilizar los conceptos de estabilidad en el sentido de Lyapunov en el contexto de disipatividad para sistemas no lineales se definen los conceptos de estado cero detectable y observable.

Sea el sistema pasivo $S$ con entrada cero, esto es $\dot{x}=f(x, 0), y=h(x, 0)$ y sea $Z \subset \mathbb{R}^{n}$ el mayor conjunto positivamente invariante contenido en $\left\{x \in \mathbb{R}^{n} \mid y=h(x, 0)=0\right\}$, podemos decir que el sistema $S$ es de estado cero detectable (ECD) si $x=0$ es condicionalmente asintóticamente estable a $Z$. Si $Z=0$, entonces $S$ es de estado cero observable (ECO).

Uno de los beneficios de la propiedad de detectabilidad es que la pasividad y la estabilidad están conectadas incluso cuando la función de almacenamiento $H(x)$ es sólo semidefinida positiva aunque el principal beneficio es que la estabilidad asintótica se logra con una realimentación simple (Sepulchre et al., 1997).

\section{Teoremas acerca de Pasividad y estabilidad}

Sea $S$ un sistema pasivo con una función de almacenamiento $H(x)$ de clase $C^{1}$, es decir, continua y diferenciable con un punto de equilibrio $x=0$, el mayor conjunto positivamente invariante $Z \subset \mathbb{R}^{n}$ contenido en $\left\{x \in \mathbb{R}^{n} \mid y=h(x, 0)=0\right\}$ y $h(x, u)$ continua y diferenciable. Entonces, se cumplen las siguientes propiedades:

- Si $H(x)$ es definido positivo, entonces el equilibrio $x=0$ de $S$ con $u=0$ es estable.

- Si el sistema $S$ es estado cero detectable (ECD), entonces el equilibrio $x=0$ de $S$ con $u=0$ es estable.

- Cuando $y=h(x)$, la realimentación $u=-y$ logra estabilidad asintótica de $x=0$ si y solo si $S$ es estado cero detectable.

\section{Demostración:}

- Si $S$ es pasivo, entonces con una entrada $u=0$, la función de almacenamiento $H(x)$ satisface $\dot{H}(x) \leq 0$. Si $H(x)$ es definido positivo, el punto de equilibrio $x=0$ de $\dot{x}=f(x, 0)$ es estable debido a que cumple con las condiciones para una función candidata de Lyapunov.

- Para comprobar la estabilidad de $x=0$ cuando la función de almacenamiento de energía $H(x)$ es solamente semidefinida positiva, entonces primero se muestra que

$$
H(x)=0 \Rightarrow h(x, 0)=0 .
$$

Puesto que $H(x) \geq 0, \forall x, \dot{H}(x) \leq u^{T} y=u^{T} h(x, u)$, debe ser no negativo para todo $u$ siempre que $H(x)=0$. Debido a que $h(x, u)$ es una función del tipo $C^{1}$ en $u$, si $y=h(x, u)=h(x, 0)+\eta(x, u) u$. Se obtiene, $\forall x \in\{x \mid H(x)=0\}$ y todo $u$

$$
0 \leq \dot{H}(x) \leq u^{T} h(x, 0)+u^{T} \eta^{T}(x, u) u .
$$

La única posibilidad de que la expresión anterior sea satisfecha para todo $u$ es que $h(x, 0)=0$ siempre que $H(x)=0$. 
Como una consecuencia, el mayor conjunto positivo invariante $Z$ de $\dot{x}=f(x, 0)$ contenido en $\{x \mid H(x)=0\}$ es también contenido en $\{x \mid h(x, 0)=0\}$. Debido a la propiedad ECD, $x=0$ es condicionalmente asintóticamente estable a $Z$, lo que prueba la estabilidad de $x=0$.

- Debido a que $h$ es independiente de $u$, el lazo de realimentación $u=-y$ está bien definido. Para $u=-y$, se satisface que

$$
\dot{H}(x) \leq-y^{T} y \leq 0 .
$$

La estabilidad es testeada como en el punto anterior.

Mediante el Principio de invariancia de Barbashin-Krasovskii-LaSalle (5.2.4), también conocido como principio de invariancia de LaSalle las soluciones acotadas de $\dot{x}=f(x, y)$ convergen al mayor conjunto invariante de $\dot{x}=f(x, 0)$ contenido en el conjunto $D$, definido previamente, $D\{x \mid h(x)=0\}$. Si $S$ es ECD, con $x=0$ se prueba la estabilidad asintótica. En cambio, si el punto de equilibrio $x=0$ del sistema $\dot{x}=f(x,-y)$, entonces es condicionalmente asintóticamente estable al subconjunto $Z$.

Finalmente, si $H(x)$ es radialmente no acotada y $\dot{H}(x) \leq 0$, entonces todas la soluciones son acotadas y las propiedades de estabilidad son globales (Sepulchre et al., 1997).

\subsection{Control basado en pasividad a partir del balance de energía}

En esta sección se presentan los conceptos que son la base del control por balance de energía para la síntesis de un controlador. Como se mencionó anteriormente, el análisis desde la perspectiva de la energía permite interpretar la dinámica de un sistema como una transformación de la energía del mismo. Esta perspectiva es particularmente útil para estudiar la dinámica de un sistema complejo no lineal. En estos últimos años la técnica de balance de energía basada en pasividad ha ganado gran popularidad y se han desarrollado diferentes aplicaciones en el área del diseño de controladores para convertidores electrónicos, sistemas electromecánicos, sistemas de levitación magnética, etc, (Ortega et al., 2001b, 2012). Además, empleando los conceptos de energía, se han diseñado observadores de estados para diferentes sistemas.

El Control Basado en Pasividad es un nombre general para definir una metodología para sintetizar un controlador que logra la estabilización haciendo pasivo al sistema con respecto a una función de energía deseada inyectando amortiguamiento. Se pueden definir dos maneras de realizar lo anterior (Ortega et al., 2002a):

- La forma clásica, que involucra definir una función de energía y sintetizar el controlador correspondiente.

- A partir de considerar una estructura de lazo cerrado deseable del sistema, se obtiene la familia de todas las funciones de energía, de las cuales debe elegirse la función adecuada, compatible con la estructura del sistema. 
En esta sección consideramos el segundo de los items, donde las aplicaciones más importantes se encuentran asociadas a los métodos de asignación de interconexión y amortiguamiento (IDA-PBC, por la sigla en inglés de Interconnection and Damping Assignment Passivity-Based Control) como así también en una técnica de control basada en pasividad llamada moldeo o balance de potencia (PS, por la sigla en inglés de Power shaping). Luego, en la etapa de diseño, a partir de las especificaciones de lazo cerrado es necesario resolver una ecuación en derivadas parciales que permite la obtención de una función de Lyapunov de una forma constructiva.

\subsubsection{Balance de energía}

Las ideas principales de la técnica de control son resumidas a continuación:

El sistema físico puede escribirse en la forma del modelo hamiltoniano según Ortega et al. (2002a)

$$
\left\{\begin{array}{l}
\dot{x}=(\mathcal{J}(x)-\mathcal{R}(x)) \frac{\partial H(x)}{\partial x}+g(x) u=f(x)+g(x) u, \\
y=g^{T}(x) \frac{\partial H(x)}{\partial x},
\end{array}\right.
$$

donde $x \in \mathbb{R}^{n}$ es el vector de estados, $u \in \mathbb{R}^{m}$ es el vector de entradas, con $m<n$, los pares $\mathcal{J}(x)$ y $g(x)$ capturan la estructura de la interconexión del sistema, con $g(x)$ modelando en particular los puertos del sistema, $\mathcal{R}(x)=\mathcal{R}(x)^{T} \geq 0$ considera la disipación del sistema en función de los estados, $H: \mathbb{R}^{n} \rightarrow \mathbb{R}$ es una función suave de los estados que representa la energía total almacenada en el sistema (Ortega et al., 2002a; Ortega y Garcia-Canseco, 2004). La matriz $\mathcal{J}(x)$ es antisimétrica $\left(\mathcal{J}(x)=-\mathcal{J}(x)^{T}\right)$ y revela la conservación de la energía en el modelo.

Dado un sistema expresado por (5.30) con una salida $g^{T}(x) \frac{\partial H(x)}{\partial x}$ es posible escribir las condiciones de pasividad para alguna función de almacenamiento de energía $H(x)$ de la siguiente forma

$$
\underbrace{H\left(x\left(t_{1}\right)\right)-H(x(0))}_{\text {Energía almacenada }} \leq \underbrace{\int_{0}^{t_{1}} u^{T}(t) y(t) d t}_{\text {Energía suministrada }} .
$$

La energía del sistema, dada por la desigualdad (5.31) no se incrementa para el sistema sin control $(u=0)$ y debido a la disipación puede decrementarse, $H[x(t)] \leq H[x(0)]$. Si la energía se encuentra acotada por debajo, el sistema se detendrá en el mínimo de energía.

La desigualdad (5.31) refleja una propiedad universal de los sistemas físicos y es de interés conservarla en sistemas de lazo cerrado. Una primera aproximación al problema es definir una función de almacenamiento de energía a lazo cerrado $H_{d}(x)$, como la suma de la función de energía del sistema a lazo abierto y de la función de energía que intercambia el controlador $H_{a}(x)$, es decir

$$
H_{d}(x)=H(x)+H_{a}(x),
$$

donde $H_{d}: \mathbb{R}^{n} \rightarrow \mathbb{R}^{+}$y $H_{a}: \mathbb{R}^{n} \rightarrow \mathbb{R}$. En un sistema de lazo cerrado es deseable asignar un nuevo punto de trabajo aislado $\left(x^{*}\right)$ para el sistema y que además se mantenga la desigualdad de balance de energía, la cual queda expresada como

$$
H_{d}\left(x\left(t_{1}\right)\right)-H_{d}(x(0)) \leq \int_{0}^{t_{1}} u^{T}(t) \tilde{y}(t) d t .
$$


Para una nueva función de salida $\tilde{y}=\tilde{h}(x)$ y una función de almacenamiento total de energía $H_{d}(x)$ definida positiva que califica como una función de Lyapunov (Astolfi et al., 2002; Jeltsema et al., 2004). La asignación del nuevo punto de trabajo puede ser lograda mediante el aporte de energía por parte del controlador del sistema $\left(H_{a}(x)\right)$.

\section{Procedimiento de diseño}

El procedimiento de diseño consiste en fijar las matrices de interconexión y de disipación manteniendo la estructura que conserva las propiedades de un sistema hamiltoniano. De acuerdo con la técnica de control de inyección de amortiguamiento, el sistema a lazo cerrado está dado por:

$$
\dot{x}=\left[\mathcal{J}_{d}(x)-\mathcal{R}_{d}(x)\right] \frac{\partial H_{d}(x)}{\partial x},
$$

donde $\mathcal{J}_{d}$ y $\mathcal{R}_{d}$ son las nuevas matrices de interconexión y de disipación que cumplen con $\mathcal{J}_{d}(x)=-\mathcal{J}_{d}^{T}(x), \mathcal{R}_{d}(x)=\mathcal{R}_{d}^{T}(x) \geq 0$ y $H_{d}: \mathbb{R}^{n} \rightarrow \mathbb{R}$ es el nuevo hamiltoniano (Batlle $\mathrm{y}$ Dòria-Cerezo, 2010).

Dado un sistema definido por la expresión

$$
\dot{x}=f(x)+g(x) u
$$

asumiendo que existe un aniquilador a izquierda $\left(g^{\perp}(x)\right)$ de $g(x)^{3}, \mathcal{J}_{d}(x)=-\mathcal{J}_{d}^{T}(x)$, $\mathcal{R}_{d}(x)=\mathcal{R}_{d}^{T}(x) \geq 0$ y una función de energía de lazo cerrado $H_{d}: \mathbb{R}^{n} \rightarrow \mathbb{R}$ resolviendo la siguiente ecuación en derivadas parciales (PDE, por su sigla en ingles "Partial Differential Equation")

$$
g^{\perp}(x) f(x)=g^{\perp}(x)\left[\mathcal{J}_{d}(x)-\mathcal{R}_{d}(x)\right] \frac{\partial H_{d}}{\partial x},
$$

es posible encontrar una función de almacenamiento de energía $H_{d}(x)$ que verifica

$$
x^{*}=\arg \min H_{d}(x)
$$

con $x^{*} \in \mathbb{R}^{n}$ el punto de equilibrio a ser estabilizado.

La PDE (5.36) resulta de igualar las expresiones (5.34) y (5.35) y de multiplicar por el aniquilador a izquierda de $g(x)$. Cabe señalar que, la ecuación en derivadas parciales (5.36) caracteriza todas las funciones de energía que pueden ser asignadas a lazo cerrado en un sistema de puertos hamiltoniano con las matrices de interconexión $\left(\mathcal{J}_{d}(x)\right)$ y de disipación $\left(\mathcal{R}_{d}(x)\right)$ dadas. Las condiciones en las que existe una ley de control para el sistema dado en (5.35) son llamadas matching conditions y están representadas por la PDE (5.36) (Ortega et al., 2002b). Entonces, el sistema a lazo cerrado toma la forma

$$
\dot{x}=\left[\mathcal{J}_{d}(x)-\mathcal{R}_{d}(x)\right] \frac{\partial H_{d}}{\partial x},
$$

con $u=\beta(x)$, donde

$$
\beta(x)=\left[g^{T}(x) g(x)\right]^{-1} g^{T}(x)\left[\mathcal{J}_{d}(x)-\mathcal{R}_{d}(x)\right] \frac{\partial H_{d}}{\partial x}-f(x) .
$$

\footnotetext{
${ }^{3}$ Tal que $g^{\perp}(x) g(x)=0$, donde el rango $\left(g^{\perp}(x)\right)=n-m$.
} 
Como se comentó previamente, si se encuentra una solución para la PDE (5.36) de tal forma que $x^{*}=\arg \min H_{d}(x)$ la estabilidad del punto de equilibrio es asegurada (Jeltsema et al., 2004).

El punto de equilibrio es asintóticamente estable si el mayor conjunto invariante a lazo cerrado contenido en

$$
\left\{x \in \mathbb{R}^{n} \mid\left[\frac{\partial H_{d}}{\partial x}\right]^{T} \quad \mathcal{R}_{d}(x) \frac{\partial H_{d}}{\partial x}=0\right\},
$$

es igual a $x^{*}$. Un estimado de su dominio de atracción es dado por el mayor conjunto acotado $x \in \mathbb{R}^{n} \mid H_{d}(x) \leq c$, con $c>0$ (Ortega y García-Canseco, 2004).

\section{Disipación pervasiva}

La técnica por balance de energía es obstaculizada por la existencia de la disipación pervasiva. Este último es un término que se refiere a la existencia de elementos disipativos cuyas potencias no se hacen cero en el punto de equilibrio deseado. En sistemas mecánicos puede ser que no esté presente esta limitación, pero en sistemas electromecánicos es común. Para analizar el problema de la disipación pervasiva se considera el sistema pasivo (5.30) con una función de almacenamiento $H(x)$ y un punto de equilibrio $x^{*}$. Es posible encontrar una función $\beta(x)$ tal que $H_{d}(x)=H(x)+H_{a}(x)$ tenga un mínimo en el punto de equilibrio $\left(x^{*}\right)$, es decir $x^{*}=\arg \min H_{d}(x)$.

La energía que intercambia el controlador con el sistema puede expresarse como:

$$
H_{a}\left(x\left(t_{1}\right)\right)-H_{a}(x(0))=\int_{0}^{t_{1}} u^{T} y d t
$$

donde el término de la derecha de la expresión anterior es la energía que entrega el controlador.

Tomando la derivada de la ecuación anterior se obtiene:

$$
\nabla H_{a}(x)^{T} \dot{x}=u^{T} y .
$$

Para construir una función $H_{a}(x)$, es necesario resolver la ecuación anterior (Ortega et al., 2001a, 2002a; Jeltsema et al., 2004) que evaluada en el punto de equilibrio $x^{*}$ es

$$
\left.\nabla H_{a}(x)^{T} \dot{x}\right|_{x=x^{*}}=u^{T} y=0 .
$$

Esta última expresión representa una condición para lograr resolver la PDE, es decir, $\dot{x}=0$ y por lo tanto $\left(u^{T} y\right)=0$ en el punto de equilibrio. Lo que quiere decir que el aporte de potencia por el controlador es nulo. Sin embargo, el producto $\left(u^{T} y\right)$, en muchas aplicaciones, no es cero en el punto de equilibrio.

A partir de los conceptos de balance de energía y con la idea de superar los problemas de la disipación pervasiva surge un nuevo concepto llamado moldeo de potencia que propone dar forma a una función de potencia que representa la dinámica del sistema mediante una función que califica como una función de Lyapunov. 


\subsection{Control basado en pasividad a partir del balance de Potencia}

En base a los estudios de Brayton-Moser (década del 60) sobre algunos circuitos eléctricos no lineales se definieron criterios de estabilidad para redes RLC que se pueden representar por una función de potencial mixto (Brayton y Moser, 1964a,b). Los conceptos anteriores y los de pasividad dieron lugar a un nuevo marco de trabajo llamado moldeo de potencia, como una alternativa a la técnica de control basada en pasividad llamada balance de energía (Ortega et al., 2003a,b). En los años sucesivos, la técnica de control por moldeo de potencia ha sido extendida a diferentes sistemas físicos, por ejemplo en sistemas mecánicos (Jeltsema y Scherpen, 2007), reactores químicos (Favache y Dochain, 2010), manipuladores robóticos (Dirksz y Scherpen, 2012a), convertidores electrónicos (Jeltsema y Scherpen, 2012; Van der Schaft y Jeltsema, 2014), etc.

\subsubsection{Modelado basado en potencia utilizando las ecuaciones de Brayton- Moser}

Las ecuaciones de Brayton-Moser están basadas en las ecuaciones diferenciales que capturan la dinámica de un circuito no lineal RLC. Brayton y Moser demostraron la existencia de una función de potencial mixto para redes eléctricas topológicamente completas, es decir para redes que pueden ser escritas por un conjunto independiente de corrientes de inductor y tensiones de capacitor. Además, la función de potencial mixto puede ser utilizada para construir una función Lyapunov (Brayton y Moser, 1964a,b). Las ecuaciones de BraytonMoser tienen la siguiente forma

$$
\begin{aligned}
& -L(i) \frac{d i}{d t}=\frac{\partial P(i, v)}{\partial i}, \\
& C(v) \frac{d v}{d t}=\frac{\partial P(i, v)}{\partial v},
\end{aligned}
$$

donde $L(i)$ y $C(v)$ son inductores y capacitores probablemente no lineales y $P(i, v)$ es una función de potencial mixto. Esta función de potencial mixto captura toda la información necesaria acerca de la estructura topológica y las características de los elementos resistivos contenidos en el circuito. La misma puede ser formada añadiendo las funciones potenciales de cada elemento que compone el sistema. Se asume que $L(i)$ y $C(v)$ son matrices definidas positivas, simétricas (Brayton y Moser, 1964a).

Las ecuaciones diferenciales que representan a un circuito eléctrico (5.44) y (5.45) pueden escribirse de forma matricial como:

$$
Q(x) \dot{x}=\frac{\partial P(x)}{\partial x},
$$

donde $Q(x)=Q(i, v)$ es una matriz de rango completo de $n \times n$ la cual contiene los valores de $C(v)$ y $L(i)$ y es dada por la siguiente expresión:

$$
Q(i, v)=\left[\begin{array}{cc}
-L(i) & 0 \\
0 & C(v)
\end{array}\right]
$$




\subsubsection{Síntesis de un controlador empleando moldeo de potencia}

Las ideas principales de los trabajos Ortega et al. (2003a,b); Jeltsema y Scherpen (2007); García-Canseco et al. (2007) y García-Canseco et al. (2010) se resumen a continuación.

Asumiendo un sistema dinámico

$$
\left\{\begin{array}{l}
\dot{x}=f(x)+g(x) u \\
y=h(x)
\end{array},\right.
$$

donde $x \in \mathbb{R}^{n}$ es el vector de estados del sistema, $u \in \mathbb{R}^{m}$ es el vector de entrada con $m \leq n$, $f(x): \mathbb{R}^{n} \rightarrow \mathbb{R}^{n}$ y $g(x): \mathbb{R}^{n} \rightarrow \mathbb{R}^{n} \times \mathbb{R}^{m}$. El sistema (5.48) puede ser descripto empleando las ecuaciones de Brayton-Moser

$$
Q(x) \dot{x}=\frac{\partial P(x)}{\partial x}+g(x) u
$$

donde $Q: \mathbb{R}^{n} \rightarrow \mathbb{R}^{n \times n}$ es una matriz de rango completo y $P(x)$ es una función de potencial mixto (García-Canseco et al., 2010). Cabe señalar que si $Q+Q^{T} \leq 0$, entonces el sistema satisface la siguiente desigualdad del balance de potencia García-Canseco et al. (2007):

$$
P(x(t))-P(x(0)) \leq \int_{0}^{t} u^{T}(\tau) \tilde{y}(\tau) d \tau,
$$

$\operatorname{con} \tilde{y}=\tilde{h}(x, u) \mathrm{y}$

$$
\tilde{h}(x, u):=-G^{T}(x) Q^{-1}(x)[\nabla P+G(x) u] .
$$

Por lo general, el punto de equilibrio de la función de potencial mixto no es un punto de trabajo de interés y es de utilidad lograr estabilizar el sistema en otro punto de equilibrio admisible. Por lo tanto, la función de potencial mixto es moldeada mediante la acción de control $u=\widehat{u}$ donde

$$
G(x) \widehat{u}=\nabla P_{a},
$$

para alguna función $P_{a}: \mathbb{R}^{n} \rightarrow \mathbb{R}$. Esto produce el siguiente sistema de lazo cerrado:

$$
Q(x) \dot{x}=\nabla P_{d}(x)
$$

donde la función total de potencia está dada por $P_{d}(x)=P(x)+P_{a}(x)$, y el punto de equilibrio puede ser estable si $x^{*}=\arg \min P_{d}(x)$. De esta manera, la potencia del sistema a lazo cerrado es balanceada mediante la diferencia entre la potencia del sistema a lazo abierto y la potencia que suministra el controlador. Además, la función total de potencia califica como una función de Lyapunov.

Por otra parte, la técnica de moldeo de potencia a diferencia de la técnica de balance de energía puede ser aplicada a sistemas con disipación pervasiva. Permitiendo incorporar elementos disipativos de una forma natural.

En contraste con el marco que brinda el hamiltoniano, una ventaja práctica del balance de potencia para los sistemas eléctricos es que su dinámica puede ser descripta por variables como tensiones y corrientes. Esto es de especial interés para realizar la realimentación del sistema ya que las variables pueden ser fácilmente medidas.

De la misma forma que el control basado en el balance de energía, el control por moldeo de potencia explota la estructura del sistema. 
Las ecuaciones que capturan la dinámica de un sistema empleando la teoría de sistemas hamiltonianos con puertos son escritas en función de las variables físicas. En consecuencia, en circuitos eléctricos que contengan elementos como inductores y capacitores es razonable modelar el sistema empleando el formalismo hamiltoniano. En el caso que el sistema incorpore fuentes de tensión controladas, fuentes de corrientes y elementos disipativos resulta más adecuado modelizar el sistema empleando las ecuaciones de Brayton-Moser.

\section{Procedimiento de síntesis}

De acuerdo a García-Canseco et al. (2007) y García-Canseco et al. (2010), las siguientes proposiciones son usadas para sintetizar un controlador empleando las ideas de moldeo de potencia:

1. Existe una matriz de rango completo $Q(x)$, no singular $(|Q(x) \neq 0|)$, que resuelve la ecuación diferencial

$$
\nabla(Q(x) f(x))=[\nabla(Q(x) f(x))]^{T},
$$

y además verifica que

$$
Q(x)+Q(x)^{T} \leq 0 .
$$

2. Existe una función escalar $P_{a}: \mathbb{R}^{n} \rightarrow \mathbb{R}$, definida positiva que en la vecindad de un punto de equilibrio $x^{*}$ verifica la siguiente ecuación en derivadas parciales

$$
g^{\perp}(x) Q^{-1}(x) \nabla P_{a}(x)=0,
$$

donde

$$
g^{\perp}(x) g(x)=0,
$$

siendo

$$
\operatorname{rank}\left(g^{\perp}(x)\right)=n-m .
$$

La elección del aniquilador a izquierda, $g^{\perp}(x)$, de la misma forma que en la técnica de moldeo de energía, puede reducir la complejidad de la solución de la expresión (5.53).

3. El punto de equilibrio $x^{*}$ es asintóticamente estable, con una función de Lyapunov $P_{d}(x)$, tal que $\left(x^{*}\right)=\arg \min P_{d}(x)$, entonces el gradiente y el Hessiano de la función $P_{d}(x)$ son:

$$
\begin{gathered}
\nabla P_{d}\left(x^{*}\right)=0 \\
\nabla^{2} P_{d}\left(x^{*}\right)>0
\end{gathered}
$$

donde la función total de potencia esta dada por

$$
P_{d}(x)=P(x)+P_{a}(x),
$$

con

$$
P(x)=\int[Q(x) f(x)]^{T} d x,
$$

además $P(x)$ satisface

$$
\nabla P(x)=Q(x) f(x) .
$$


Bajo estas condiciones, la ley de control

$$
u_{P S}=\left[g^{T} Q^{T} Q g\right]^{-1} g^{T} Q^{T} \nabla P_{a},
$$

asegura que el punto de equilibrio $x^{*}$ es asintóticamente estable y una estimación de su dominio de atracción es dada por

$$
\left\{x \in \mathbb{R}^{n} \mid P_{d}(x) \leq c\right\}
$$

con $c>0$.

\section{Demostración}

Para probar estabilidad se define la matriz $G(x)$ y su aniquilador a izquierda de rango completo de la siguiente forma

$$
G(x):=Q(x) g(x)
$$

y

$$
G^{\perp}(x):=g^{\perp}(x) Q^{-1}(x) .
$$

Con estas dos matrices se define una tercera matriz de rango completo como

$$
G_{1}(x)=\left[\begin{array}{l}
G^{\perp}(x) \\
G^{T}(x)
\end{array}\right] .
$$

La primera prueba consiste en mostrar que bajo la suposición (5.51) y (5.52) es posible escribir el sistema dinámico (5.48) en la forma de las ecuaciones de Brayton - Moser (5.49). Invocando el lema de Poincaré se obtiene que la expresión (5.51) es equivalente a la existencia de una función de potencial mixto $P: \mathbb{R}^{n} \rightarrow \mathbb{R}$ de forma tal que

$$
Q f=\nabla P .
$$

Entonces, multiplicando a izquierda la expresión (5.66) por la ecuación anterior y teniendo en cuenta que $P=P_{d}-P_{a}$ se obtiene

$$
\left[\begin{array}{c}
G^{\perp} \\
G^{T}
\end{array}\right] Q \dot{x}=\left[\begin{array}{c}
G^{\perp}\left(\nabla P_{d}-\nabla P_{a}\right) \\
G^{T}\left(\nabla P_{d}-\nabla P_{a}\right)+G^{T} G u
\end{array}\right] .
$$

Sustituyendo la acción de control, dada por (5.61), en la expresión anterior y empleando la matching condition $g^{\perp} Q(x)^{-1} \nabla P_{a}(x)=0$, finalmente llegamos a la siguiente ecuación de lazo cerrado $Q \dot{x}=\nabla P_{d}$.

Entonces, tomando la derivada en función del tiempo alrededor de la dinámica de lazo cerrado se obtiene

$$
\dot{P}_{d}=\frac{1}{2} \dot{x}^{T}\left(Q+Q^{T}\right) \dot{x} .
$$

Teniendo en cuenta que $P_{d}(x)$ califica como una función candidata de Lyapunov y observando la expresión anterior, donde debe ser $\dot{P}_{d} \leq 0$, entonces se puede concluir que se tiene que cumplir $Q+Q^{T} \leq 0$. Aplicando el principio de invariancia de LaSalle es posible demostrar que la estabilidad del sistema es asintótica. 
Una desventaja de la aplicación de las técnicas de control tanto por moldeo de potencia como de balance de energía es que es necesario resolver una ecuación en derivadas parciales que no siempre es fácil. En un trabajo reciente, la necesidad de resolver una ecuación en derivadas parciales aplicando balance de energía para una clase de sistemas mecánicos es evitada (Donaire et al., 2015).

\subsubsection{Representación alternativa de los pares $Q(x), P(x)$}

En el caso de no disponer de una matriz $Q(x)$ que cumpla con las condiciones $\left(\nabla(Q(x) f(x))=[\nabla(Q(x) f(x))]^{T}\right)$ y $\left(Q(x)+Q(x)^{T} \leq 0\right)$, es posible proponer un nuevo par $(\tilde{Q}(x), \tilde{P}(x))$ que represente de forma equivalente la dinámica del sistema. De esta manera, con estos nuevos pares $\tilde{Q}(x)$ y $\tilde{P}(x)$ es posible aplicar la técnica de moldeo de potencia para sintetizar un controlador que explote las características del sistema.

El nuevo par $\tilde{Q}(x)$ y $\tilde{P}(x)$ representa el sistema de forma equivalente a las expresiones de Brayton-Moser (5.49) siendo

$$
\tilde{Q} \dot{x}=\nabla \tilde{P}+\tilde{G} u
$$

donde

$$
\tilde{Q}(x)=\left[\frac{1}{2} \nabla(Q f) M+\frac{1}{2} \nabla^{T}(M Q f)+\lambda I\right] Q,
$$

donde $\tilde{Q}(x)$ también cumple con

$$
\nabla(\tilde{Q}(x) f(x))=[\nabla(\tilde{Q}(x) f(x))]^{T},
$$

$\mathrm{y}$

$$
\tilde{Q}(x)+\tilde{Q}(x)^{T} \leq 0,
$$

$\tilde{P}(x)$ es dado por

$$
\tilde{P}(x)=\lambda \int(Q f)^{T} d x+\frac{1}{2} f^{T} Q^{T} M Q f,
$$

$\mathrm{y}$

$$
\tilde{G}=\tilde{Q} g .
$$

donde $\lambda \in \mathbb{R}$ y $M=M^{T}: \mathbb{R}^{n} \rightarrow \mathbb{R}^{n x n}$, son arbitrarias (Jeltsema, 2005; García-Canseco et al., 2007). La solución de la ecuación en derivadas parciales, (5.53), nos permite diseñar una función $P_{a}(x)$.

\section{Equivalencias entre formalismos}

Existe una relación entre la estructura hamiltoniana y las ecuaciones de Brayton-Moser. De esta manera, es posible plantear una equivalencia entre los dos formalismos. Para un sistema hamiltoniano dado por las expresiones (5.30). Con una matriz de rango completo $\mathcal{J}(x)-\mathcal{R}(x)$ una solución trivial de $\nabla(Q(x) f(x))=[\nabla(Q(x) f(x))]^{T}$ es obtenida configurando $Q(x)$ como:

$$
Q(x)=[\mathcal{J}(x)-\mathcal{R}(x)]^{-1} .
$$

Aunque, en tal caso la función potencial asociada no es modificada y se mantiene la energía total almacenada en vez de la potencia como es deseado (Jeltsema y Scherpen, 2003; García-Canseco et al., 2007). 


\subsubsection{Balance de potencia con Control Integral}

Es bien conocida la capacidad de los controladores con acción integral para compensar errores de estado estacionario causados por perturbaciones o incertezas en el modelo. En un sistema de puertos hamiltonianos es posible agregar una acción integral en un sistema pasivo en la salida pasiva de forma tal que la estabilidad del sistema a lazo cerrado es preservada. A continuación resumimos la adición de una acción de control integral en un sistema hamiltoniano de puertos expresado como

$$
\text { Sistema }\left\{\begin{array}{l}
\dot{x}=F(x) \nabla H+g(x)\left(u+d_{1}\right) \\
y=g^{T}(x) \nabla H(x)
\end{array},\right.
$$

en la salida pasiva con una perturbación constante $d_{1} \in \mathbb{R}^{m}$, donde $x \in \mathbb{R}^{n}, u \in \mathbb{R}^{m}$, $g: \mathbb{R}^{n} \rightarrow \mathbb{R}^{n x m}, H: \mathbb{R}^{n} \rightarrow \mathbb{R}$ es la función de energía total y por una cuestión de claridad en la explicación se define $F(x)=\mathcal{J}(x)-\mathcal{R}(x)$, donde se cumple que $F(x)+F(x)^{T} \leq 0$. Se agrega una acción integral con el objetivo de hacer cero la salida pasiva tomada como un error (Ortega y García-Canseco, 2004; Ortega y Romero, 2011):

$$
\begin{aligned}
& \dot{\eta}=k_{i} y, \\
& u=-\eta,
\end{aligned}
$$

donde $k_{i}$ es definida positiva. El sistema a lazo cerrado con el control integral queda representado como

$$
\left[\begin{array}{c}
\dot{x} \\
\dot{\eta}
\end{array}\right]=\left[\begin{array}{cc}
F(x) & g(x) k_{i} \\
-k_{i} g(x)^{T} & 0
\end{array}\right] \nabla W(x, \eta),
$$

donde $W(x, \eta)$ es una nueva función candidata de Lyapunov definida como

$$
W(x, \eta):=H(x)+\frac{1}{2}\left(\eta-d_{1}\right)^{T} k_{i}^{-1}\left(\eta-d_{1}\right) .
$$

La adición de una acción integral al sistema hamiltoniano de puertos conserva la estructura y la demostración de estabilidad puede llevarse a cabo empleando el teorema de LaSalle (Ortega y Romero, 2011). Otra posibilidad de plantear un control integral en un sistema hamiltoniano preservando la estructura del hamiltoniano, en el cual se plantea un cambio de coordenadas es reportado en (Donaire y Junco, 2009).

Empleando la equivalencia entre formalismos citada anteriormente para puertos hamiltonianos y las ecuaciones de BM es posible agregar un control integral a este último. Se considera un control con acción integral con una perturbación constante que en lazo cerrado retenga la estructura de las ecuaciones de Brayton-Moser.

Las ecuaciones de Brayton-Moser (5.49) pueden ser escritas como

$$
Q(x) \dot{x}=\frac{\partial P}{\partial x}+g(x)(u-d),
$$

donde $d$ es la perturbación constante.

Entonces, la acción de control (Dirksz y Scherpen, 2010):

$$
u=u_{P S}+\tau,
$$


estabiliza el sistema (5.80) asintóticamente, siendo $u_{P S}$ la acción de control diseñada por moldeo de potencia con una nueva función de potencia total $P_{d}$ y $\tau$ el estado integral con dinámica

$$
\dot{\tau}=-K_{i} g^{T}(x) \tilde{Q}^{-1}(x) \frac{\partial P_{d}(x)}{\partial x},
$$

empleando una representación alternativa para la matriz $Q(x)$, donde la nueva función total de potencia esta dada por

$$
\tilde{P}_{i}(x)=P_{d}(x)+\frac{1}{2} \bar{\tau}^{T} K_{i}^{-1} \bar{\tau},
$$

con una constante definida positiva $K_{i}$ y $\bar{\tau}=\tau-d$.

Para verificar que la adición del integrador al sistema de lazo cerrado conserva la estructura de Brayton Moser es posible escribir

$$
\left[\begin{array}{cc}
\tilde{Q}(x) & 0 \\
0 & I
\end{array}\right]\left[\begin{array}{c}
\dot{x} \\
\dot{\bar{\tau}}
\end{array}\right]=\left[\begin{array}{cc}
I & \tilde{Q}(x) Q^{-1}(x) g(x) K_{i} \\
-K_{i} g^{T}(x) Q^{-T}(x) & 0
\end{array}\right]\left[\begin{array}{c}
\frac{\partial \tilde{P}_{i}}{\partial x} \\
\frac{\partial \tilde{P}_{i}}{\partial \bar{\tau}}
\end{array}\right] .
$$

Luego

$$
\left[\begin{array}{c}
\dot{x} \\
\dot{\bar{\tau}}
\end{array}\right]=\left[\begin{array}{cc}
\tilde{Q}^{-1}(x) & Q^{-1}(x) g(x) K_{i} \\
-K_{i} g^{T}(x) Q^{-T}(x) & 0
\end{array}\right]\left[\begin{array}{c}
\frac{\partial \tilde{P}_{i}}{\partial x} \\
\frac{\partial \tilde{P}_{i}}{\partial \bar{\tau}}
\end{array}\right] .
$$

Se puede observar que la última expresión y (5.78) tienen la misma estructura, con lo cual las propiedades de las ecuaciones de Brayton-Moser se mantienen con la adicción de la acción integral. Se puede observar que $K_{i} g^{T}(x) Q^{-T}(x) \tilde{Q}(x) Q^{-1}(x) g K_{i}$ es diferente a cero, por lo cual la matriz (5.84) es no singular. De esta manera se prueba que (5.84) puede ser invertible y da una descripción de Brayton Moser del sistema de lazo cerrado (Dirksz y Scherpen, 2012b).

Esta metodología para agregar un control integral en un sistema que ya posee un controlador calculado por moldeo de potencia resulta de interés debido a que no es necesario resolver una nueva PDE para obtener una acción de control.

En este caso se considera una perturbación constante en el sistema. La adición de un control integral con una perturbación desconocida en la salida (pasiva/ no pasiva) puede realizarse empleando una transformación de coordenadas. Este nuevo diseño puede llevarse a cabo mediante una transformación de coordenadas (Ortega y Romero, 2011, 2012; Romero et al., 2013).

\subsection{Retrospección}

En este capítulo se presentó, en la primera parte, la teoría de pasividad aplicada al control de sistemas. También se comentó la estabilidad de un sistema en el sentido de Lyapunov.

Se realizó un resumen con las principales ideas de los formalismos de control basado en la energía y en la potencia. Además, se han presentado las ventajas y desventajas de cada técnica.

El control basado en pasividad, llamado de moldeo de potencia preserva la estructura de las ecuaciones de Brayton-Moser. Esta preservación de la estructura permite reutilizar los resultados de Brayton-Moser para estudiar la estabilidad del sistema controlado. Además, la 
modelización de un sistema eléctrico se lleva a cabo empleando variables como tensiones y corrientes. Estas variables se pueden medir a través de sensores comunes. Lo cual nos permite una implementación sencilla de la ley de control.

También se presentó, en la sección de moldeo de potencia, una técnica para agregar un control integral preservando la estructura de las ecuaciones de Brayton-Moser. Este resultado es importante ya que la adición de un control integral fue propuesto por Ortega y co-autores para un formalismo y luego estos conceptos fueron extrapolados para aplicarlos a un sistema modelado con las ecuaciones de Brayton-Moser. 


\title{
Capítulo 6
}

\section{Control por moldeo de potencia en Generación Distribuida RenOVABLE}

\section{Resumen}

\begin{abstract}
Este capítulo se centra en presentar las aplicaciones desarrolladas en el trabajo de tesis. Se aplica la técnica de control basada en conceptos de moldeo de potencia a diferentes problemas referidos a sistemas de generación de energía renovable. En particular, se desarrolla un control para la potencia activa y reactiva de un aerogenerador de 1,5 MW conectado a una red eléctrica. Adicionalmente, utilizando las ideas del aerogenerador equivalente se realiza el control de un parque eólico con seis aerogeneradores. Finalmente, se controla un sistema de almacenamiento de energía en el contexto de una micro red.
\end{abstract}

\subsection{Introducción}

En los últimos años ha aumentado vertiginosamente la potencia instalada de generación de energía eólica como así también la potencia nominal de las unidades de generación. En función de la creciente demanda de energía y el renovado interés en las energías renovables se espera que esta tendencia se mantenga incrementando considerablemente la capacidad eólica instalada a nivel mundial aún con los recientes cambios en el precio del petróleo (EWEA, 2014; GWEC, 2014).

Históricamente los aerogeneradores han sido predominantemente operados para maximizar la extracción de la energía eléctrica. Sin embargo, en estas últimas décadas los sistemas de conversión de energía eólica son operados con el objetivo de lograr una buena integración a la red (Bianchi et al., 2006). 
En general, los sistemas de generación distribuida que emplean energías renovables como fuentes de generación eléctrica operan conectados a una red de distribución, siendo los valores de tensión y frecuencia impuestos por la misma. La conexión de sistemas de conversión de energía renovable en redes de distribución debe cumplir con los códigos de red para asegurar la calidad de la potencia y también deben compartir la responsabilidad de garantizar la demanda de potencia de la red de distribución. En muchos casos, cuando el sistema está trabajando de forma aislada los valores de tensión y de frecuencia pueden ser provistos por un generador sincrónico impulsado por un motor diésel, mientras que los sistemas de generación distribuida renovable (GDR) pueden aportar potencia con el objetivo de minimizar el consumo de combustible por parte del impulsor.

Los sistemas de generación distribuida renovable presentan características y fenómenos difíciles de predecir y/o modelar mediante las herramientas tradicionales de análisis y control. En particular, los sistemas de GDR son no lineales y están sometidos a la variabilidad no solo de la carga, sino también del recurso eólico. Luego, la aplicación de técnicas convencionales (PI, PID) no siempre pueden garantizar un buen desempeño en todo el rango de operación del sistema de conversión.

Los sistemas de GDR se estructuran sobre la base de una conexión a la red eléctrica preexistente, la cual generalmente resulta ser débil. Esto último puede introducir importantes restricciones cuando la inyección local de potencia es fluctuante por razones inherentes al mismo recurso renovable. En este sentido, la utilización de técnicas de control avanzadas como moldeo de potencia (PS, por su sigla en inglés Power Shaping) o de ganancias programadas $\mathcal{H}_{\infty}$ pueden dar mayores garantías para mejorar el comportamiento del sistema (Muhando et al., 2011).

Recientemente, las micro redes han sido objeto de estudio debido a la creciente demanda de energía eléctrica confiable, segura, eficiente, limpia y sustentable. Como se comentó en el capítulo relacionado a redes eléctricas, podemos decir que una micro red está compuesta por un conjunto de cargas interconectadas y diferentes sistemas de generación de energía distribuidos con el objetivo de operar como si fuese una sola unidad de generación. Las micro redes son subsistemas autónomos con sistemas de control que tienen como objetivo asegurar la calidad de la energía para las cargas conectadas a la misma (Bidram et al., 2014). Además, una de las características sobresalientes de una micro red es que puede operar conectada a la red de distribución y de forma aislada. Cuando la micro red se encuentra conectada a la red de distribución las unidades de generación, si tienen capacidad de suministrar más potencia, pueden aportarla al punto de conexión. Si las unidades de generación están basadas en energías renovables el aporte de potencia depende de la disponibilidad del recurso renovable.

Por otra parte, cuando el recurso renovable es escaso, la red de distribución puede asistir a la micro red aportando potencia. Adicionalmente, en el modo conectado a red los valores de tensión y frecuencia son impuestos por la red de distribución. En el caso de que exista una falla en la red de distribución la micro red se puede desconectar de la red de distribución y es capaz de operar de forma autónoma hasta que se solucione el desperfecto en la red de distribución.

Los sistemas de generación distribuida renovable pueden formar parte de los sistemas de generación de energía de una micro red con una alta penetración. Además, con la inclusión de sistemas de generación renovable, en algunas topologías se agregan sistemas de almacenamiento de energía con el fin de amortiguar variaciones rápidas de potencia o incluso para entregar energía por un tiempo más prolongado. El dimensionamiento del 
sistema de almacenamiento de energía depende de las características de compensación que debe incorporar al sistema eléctrico de potencia (Barton y Infield, 2005).

Con el objetivo de estudiar el control de una pequeña red eléctrica mediante técnicas avanzadas de control, como son las técnicas basadas en pasividad y empleando un enfoque deductivo para realizar un análisis del problema, primero se analizó el control de un aerogenerador equipado con un generador de rotor bobinado (DFIG). En este sentido, se desarrolló un controlador para la potencia activa y reactiva de un aerogenerador de 1,5 MW conectado a una red eléctrica (Peña et al., 2013; Peña et al., 2014). Además, empleando los conceptos de un aerogenerador equivalente se propuso el control de un grupo de 6 aerogeneradores conectados a una red eléctrica (Peña et al., 2014). Posteriormente, como parte de la actividades que dan lugar a este trabajo, se analizó una estructura de una micro red como la que se propuso en el trabajo Toccaceli et al. (2012b) donde el control propuesto gobierna el sistema de almacenamiento de energía para tratar de mitigar variaciones de potencia activa y reactiva producidas por variaciones de la carga o por variaciones del recurso eólico.

\subsection{Control de aerogeneradores con conexión a red}

El control de la potencia activa es utilizado para balancear la demanda de energía, la cual contribuye a la estabilidad de frecuencia del sistema de potencia, mientras que el control de la potencia reactiva es comúnmente utilizado en la regulación de tensión en el punto de conexión del sistema de generación. En este trabajo se considera un aerogenerador equipado con un generador DFIG operando a velocidad variable. Este tipo de generador es uno de los más empleados en parques eólicos y además la operación a velocidad variable ofrece ventajas significativas cuando se compara con generadores que operan a velocidad fija. Estas ventajas incluyen mejor desempeño en el control de la potencia activa y reactiva inyectada en el punto de conexión. La velocidad de la máquina puede variar en $\pm 30 \%$ alrededor de la velocidad sincrónica y la regulación de la velocidad es esencial para la operación óptima del sistema eólico. Por otra parte este tipo de generadores, adecuadamente controlados, tienen la capacidad de permanecer vinculados a la red en el caso que exista una falla.

En la Figura 6.1 se presenta un esquema de un aerogenerador conectado a la red, equipado con un generador de rotor bobinado, los convertidores RSC y GSC y su sistema de control. Como se mencionó en los capítulos anteriores el estator de la máquina se encuentra unido a la red y el rotor al RSC. El vínculo entre la turbina eólica y el generador asincrónico se lleva a cabo mediante una caja multiplicadora que adapta las velocidades de la turbina y del generador.

El seguimiento de la velocidad se lleva a cabo con el objetivo de extraer el máximo de potencia disponible en el viento teniendo en cuenta la curva de potencia de la turbina. Este objetivo es logrado a partir del control del convertidor conectado al rotor del generador DFIG. Por lo tanto, se necesita un diseño eficaz del controlador del convertidor RSC para cumplir con los objetivos señalados previamente.

Generalmente, para el control de la máquina se utiliza control vectorial. Diferentes marcos de referencia pueden ser utilizados para el control del DFIG, como por ejemplo control de campo orientado o control orientado según el flujo de red (Petersson et al., 2004).

En este trabajo de tesis se optó por utilizar la técnica de control orientado según el flujo de 


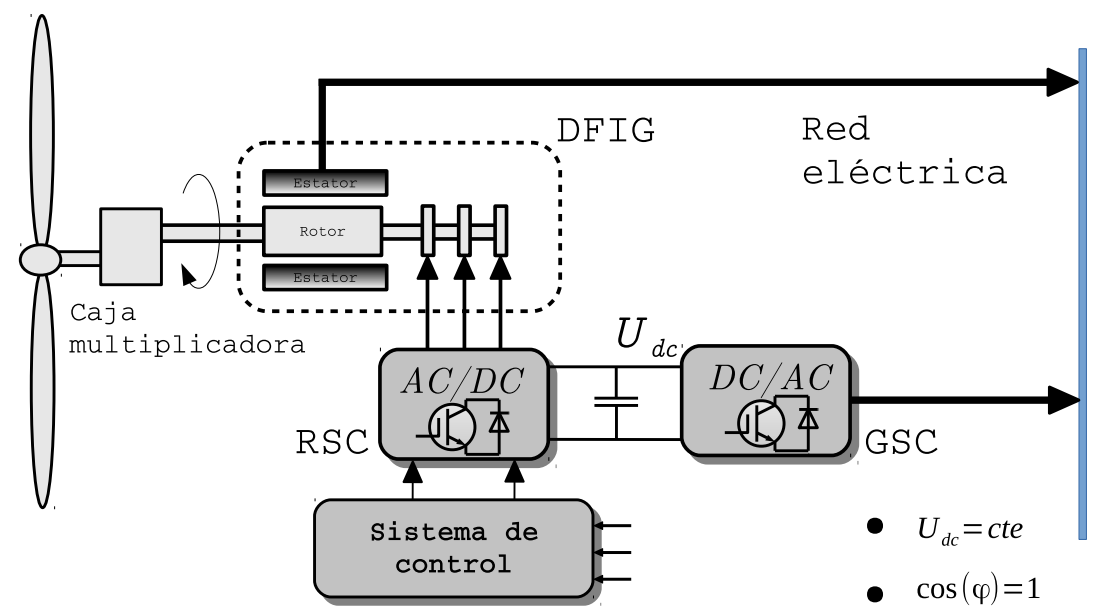

Figura 6.1: Sistema de conversión con generador asincrónico de rotor bobinado conectado a una red de distribución.

red, debido a las ventajas enumeradas en el capítulo de control vectorial. Como resultado de la utilización de la técnica de control vectorial se pueden obtener dos variables desacopladas entre sí que corresponden a las tensiones del rotor. Este desacoplamiento permite el control de forma independiente de las potencias activa y reactiva que aporta el generador al sistema de potencia.

\subsubsection{Modelo dinámico del aerogenerador}

El sistema de la Figura 6.1 se representa con un modelo de estados:

$$
\begin{aligned}
& \dot{x}=f(x)+g(x) u \\
& y=h(x)
\end{aligned}
$$

Las expresiones de la tensión en el estator y en el rotor dadas en el capítulo referido a control vectorial pueden simplificarse de la siguiente forma:

$$
\begin{gathered}
\bar{u}_{s g}=R_{s} \bar{i}_{s g}+j \omega \bar{\lambda}_{s g}, \\
\bar{u}_{r g}=R_{r} \bar{i}_{r g}+j\left(\omega-\omega_{r}\right) \bar{\lambda}_{r g}+\frac{d \bar{\lambda}_{r g}}{d t} .
\end{gathered}
$$

Reemplazando en la ecuación anterior por el valor del flujo en el rotor en un marco de referencia general, $\bar{\lambda}_{r g}=L_{r} \bar{i}_{r g}+L_{m} \bar{i}_{s g}$, se obtiene:

$$
\bar{u}_{r g}=R_{r} \bar{i}_{r g}+j\left(\omega-\omega_{r}\right)\left(L_{r} \bar{i}_{r g}+L_{m} \bar{i}_{s g}\right)+\frac{d}{d t}\left(L_{r} \bar{i}_{r g}+L_{m} \bar{i}_{s g}\right) .
$$

La tensión en el rotor en un marco de referencia general puede escribirse, como:

$$
\bar{u}_{r g}=R_{r} \bar{i}_{r g}+L_{r} \frac{d i_{r g}}{d t}+u_{d},
$$


donde

$$
u_{d}=j\left(\omega-\omega_{r}\right)\left(L_{r} \bar{i}_{r g}+L_{m} \bar{i}_{s g}\right)+L_{m} \frac{d \bar{i}_{s g}}{d t} .
$$

La expresión anterior permite trabajar con un sistema lineal debido a que $u_{d}$, en la implementación del controlador, simplemente se puede sumar a la tensión del rotor. Además, todas las cantidades de la ecuación (6.6) son valores medibles.

Las expresiones de la derivada de la corriente en el rotor de la máquina de inducción en un marco de referencia general según la tensión $(x, y)$ son:

$$
\begin{aligned}
\frac{d i_{r x}}{d t} & =-\frac{R_{r}}{L_{r}} i_{r x}+\frac{1}{L_{r}} u_{r x}, \\
\frac{d i_{r y}}{d t} & =-\frac{R_{r}}{L_{r}} i_{r y}+\frac{1}{L_{r}} u_{r y} .
\end{aligned}
$$

Por otro lado la aceleración angular del eje del rotor del generador está dada por:

$$
\frac{d \omega_{r}}{d t}=\frac{n p}{J}\left(T_{e}-B_{r} \omega_{r}-T_{m}\right)
$$

donde $n p$ es el número de pares de polos, $J$ es la inercia del sistema, $B_{r}$ es el coeficiente de fricción de la carga, $\omega_{r}$ es la velocidad angular del rotor y por último $T_{e}$ y $T_{m}$ son los pares electromagnético y mecánico impulsor, respectivamente.

El par electromagnético está dado por:

$$
T_{e}=\frac{3}{2} n p\left(\lambda_{s x} i_{s y}-\lambda_{s y} i_{s y}\right)
$$

y el par impulsor de la turbina eólica fue definido en el capítulo referido al recurso eólico y está dado por:

$$
T_{m}=\frac{1}{2} \pi \rho R^{3} \frac{C_{p}(\lambda, \beta)}{\lambda} v^{2},
$$

donde $R$ es la longitud de la pala, $v$ es la velocidad del viento, $C_{p}(\lambda, \beta)$ es el coeficiente de potencia que depende de $\lambda=\frac{\Omega R}{v}=\frac{\omega_{r} R}{k_{c} v}$, con $k_{C}$ la relación de transmisión de la caja multiplicadora y el ángulo de paso de la pala $\beta$.

A partir de las ecuaciones de las derivadas de las corrientes en el rotor (6.7), (6.8) y la ecuación mecánica de la aceleración angular (6.9) es posible escribir las ecuaciones del sistema de acuerdo la expresión (6.1):

$$
\dot{x}=\left(\begin{array}{l}
-\frac{R_{r}}{L_{r}} x_{1} \\
-\frac{R_{r}}{L_{r}} x_{2} \\
2\left(-\frac{B_{r} x_{3}}{J}-\frac{3 U x_{2} L_{m}}{J \omega L_{s}}\right)
\end{array}\right)+\left(\begin{array}{ll}
0 & \frac{1}{L_{r}} \\
\frac{1}{L_{r}} & 0 \\
0 & 0
\end{array}\right) u
$$

donde $x^{T}=\left[i_{r x}, i_{r y}, \omega_{r}\right]$.

Con el objetivo de diseñar un controlador para un DFIG, la dinámica asociada a los convertidores "RSC" puede no ser tenida en cuenta, debido a que estos pueden modificar la corriente muy rápidamente, aproximadamente en el orden de medio ciclo de red (Peña et al., 1996). 


\subsubsection{Control por moldeo de potencia}

Para diseñar un controlador empleando la técnica de control por moldeo de potencia para un sistema dinámico (6.1), es necesario representar al mismo empleando las ecuaciones de Brayton-Moser en la forma:

$$
Q(x) \dot{x}=\nabla P+G(x) u .
$$

Como se comentó en el capítulo referido a la técnica de control basada en pasividad utilizando moldeo de potencia, existen condiciones para la matriz $Q(x)$, la cual debe ser de rango completo y debe cumplir con:

$$
\nabla(Q(x) f(x))=[\nabla(Q(x) f(x))]^{T},
$$

además,

$$
Q(x)+Q(x)^{T} \leq 0 .
$$

De forma genérica, la matriz $Q(x)+Q(x)^{T}$ tiene la forma:

$$
Q+Q^{T}=\left[\begin{array}{ccc}
2 q_{11} & q_{12}+q_{21} & q_{13}+q_{31} \\
q_{12}+q_{21} & 2 q_{22} & q_{23}+q_{32} \\
q_{13}+q_{31} & q_{23}+q_{32} & 2 q_{33}
\end{array}\right]
$$

El producto de las matrices $Q(x) f(x)$ es:

$$
Q f=\left[\begin{array}{l}
-q_{11} \frac{R_{r}}{L_{r}} x_{1}-q_{12} \frac{R_{r}}{L_{r}} x_{2}+q_{13} \frac{-T_{m}-B_{r} x_{3}-\frac{3 U x 2 L_{m}}{w L_{s}}}{J} \\
-q_{21} \frac{R_{r}}{L_{r}} x_{1}-q_{22} \frac{R_{r}}{L_{r}} x_{2}+q_{23} \frac{-T_{m}-B_{r} x_{3}-\frac{3 U x 2 L_{m}}{w L_{s}}}{J} \\
-q_{31} \frac{R_{r}}{L_{r}} x_{1}-q_{32} \frac{R_{r}}{L_{r}} x_{2}+q_{33} \frac{-T_{m}-B_{r} x_{3}-\frac{3 U x 2 L_{m}}{w L_{s}}}{J}
\end{array}\right] .
$$

Luego, la matriz $\nabla Q f$ es:

$$
\left[\begin{array}{ccc}
-q_{11} \frac{R_{r}}{L_{r}} & -q_{21} \frac{R_{r}}{L_{r}} & -q_{31} \frac{R_{r}}{L_{r}} \\
-q_{12} \frac{R_{r}}{L_{r}}-3 q_{13} \frac{U L_{m}}{J w L_{s}} & -q_{22} \frac{R_{r}}{L_{r}}-3 q_{23} \frac{U L_{m}}{J w L_{s}} & -q_{32} \frac{R_{r}}{L_{r}}-3 q_{33} \frac{U L_{m}}{J w L_{s}} \\
-q_{13} \frac{B_{r}}{J} & -q_{23} \frac{B_{r}}{J} & -q_{33} \frac{B_{r}}{J}
\end{array}\right]
$$

Con la finalidad de encontrar una matriz $Q(x)$ que represente el sistema dinámico (6.12) es posible construir un sistema de ecuaciones empleando la condición (6.14). Observando el elemento 1,2 de la matriz $\nabla Q f(1,2)$ y comparando con el elemento de 1,2 de la matriz $\nabla[Q f]^{T}(1,2)$ se obtiene

$$
\nabla Q f(1,2)=[\nabla(Q f)]^{T}(1,2) \Rightarrow-q_{21} \frac{R_{r}}{L_{r}}=-\left(q_{12} \frac{R_{r}}{L_{r}}+3 q_{13} \frac{U L_{m}}{J w L_{s}}\right) .
$$

De igualar los elementos 1,3 de las matriz (6.18) y su transpuesta resulta

$$
\nabla Q f(1,3)=[\nabla(Q f)]^{T}(1,3) \Rightarrow q_{31} \frac{R_{r}}{L_{r}}=q_{13} \frac{B_{r}}{J} .
$$

Aplicando un procedimiento similar para el elemento 2,3 se obtiene

$$
\nabla Q f(2,3)=[\nabla(Q f)]^{T}(2,3) \Rightarrow q_{32} \frac{R_{r}}{L_{r}}+3 q_{33} \frac{U L_{m}}{J w L_{s}}=q_{23} \frac{B_{r}}{J} .
$$


Para reducir el número de ecuaciones involucradas en la expresión (6.14), los siguientes valores para los elementos de $Q(x)$ son adoptados:

$$
q_{21}=0, q_{13}=0, q_{23}=0 .
$$

De esta manera, observando la expresión (6.21) los elementos $q_{31}=0$ y $q_{12}=0$. De la ecuación (6.21) se puede despejar el valor del elemento $q_{32}$ :

$$
q_{32}=-q_{33} 3 \frac{U L_{m} L_{r}}{J w L_{s} R_{r}} .
$$

Luego, la matriz $Q(x)$ es:

$$
Q(x)=\left[\begin{array}{ccc}
q_{11} & 0 & 0 \\
0 & q_{22} & 0 \\
0 & q_{32} & q_{33}
\end{array}\right] .
$$

Una vez definida la matriz $Q(x)$, se eligen los valores de cada elemento considerando:

$$
Q(x)+Q(x)^{T}=\left[\begin{array}{ccc}
2 q_{11} & 0 & 0 \\
0 & 2 q_{22} & q_{32} \\
0 & q_{32} & 2 q_{33}
\end{array}\right] \leq 0 .
$$

El primer menor principal de la matriz $Q(x)+Q^{T}(x)$ es:

$$
A_{1}=2 q_{11} \leq 0,
$$

entonces, $q_{11}=-1$ comprueba la condición. A partir de la misma metodología, los menores principales que cumplen con la condición indicada son:

$$
\begin{gathered}
q_{22}=-\frac{18}{6}\left(\frac{U L_{m} L_{r}}{J R_{r} w L_{s}}\right)^{2}, \\
q_{32}=-q_{33} 3 \frac{U L_{m} L_{r}}{J w L_{s} R_{r}},
\end{gathered}
$$

y $q_{33}=-1$.

Luego, la matriz $Q(x)$, que cumple con las condiciones (6.14) y (6.15) está dada por:

$$
Q(x)=\left[\begin{array}{ccc}
-1 & 0 & 0 \\
0 & -\frac{18}{6}\left(\frac{U L_{m} L_{r}}{J R_{r} w L_{s}}\right)^{2} & 0 \\
0 & 3 \frac{U L_{m} L_{r}}{J w L_{s} R_{r}} & -1
\end{array}\right] .
$$

\section{Determinación de la función de energía mediante la solución de una PDE}

El balance de potencia resulta de definir una función de potencia total $P_{d}(x)$ como la suma de la función de potencial mixto $P(x)$ y la potencia que entrega el controlador $P_{a}(x)$ de la siguiente forma:

$$
P_{d}(x)=P(x)+P_{a}(x) .
$$


El punto de equilibrio es estable si $x^{*}=\arg \min P_{d}\left(x^{*}\right)$. La PDE a resolver fue presentada en el Capítulo 5 y está dada por:

$$
g^{\perp}(x) Q^{-1}(x) \nabla P_{a}(x)=0,
$$

donde $g^{\perp}(x)$ es el aniquilador a izquierda de $g(x)$, es decir, $g^{\perp}(x) g(x)=0$. Además, como se comentó en el capítulo referido a la técnica de control basada en el balance de energía, la PDE (6.31) caracteriza todas las funciones admisibles de energía a lazo cerrado con el sistema descripto por las ecuaciones de Brayton-Moser. La solución de la expresión (6.31) permite diseñar una función $P_{a}(x)$ que moldea la función de potencial mixto mediante el control de forma tal que $x^{*}=\arg \min \left[P_{d}(x)\right]$.

Tal como han sido desarrolladas las actividades que dan lugar a este trabajo de tesis, respecto del control de aerogeneradores, en primer lugar se propuso un controlador para la potencia activa de un DFIG (Peña et al., 2014) y en segundo término se propuso uno que tiene la capacidad de controlar las potencias activa y reactiva de un aerogenerador (Peña et al., 2013; Peña et al., 2015).

\subsubsection{Control de la potencia activa}

Para el primer caso analizado se plantea el control de la potencia activa de un aerogenerador equipado con un DFIG, entonces, eligiendo un marco de referencia orientado según la tensión de línea las potencias activas y reactivas quedan desacopladas entre sí como se comentó previamente en el Capítulo 4. La matriz $g(x)$ para el control de la potencia activa es:

$$
g(x)=\left[\begin{array}{c}
0 \\
\frac{1}{L_{r}} \\
0
\end{array}\right]
$$

La elección del aniquilador a izquierda (6.33) no es única y dicha elección aporta un grado más de libertad a la hora de resolver la PDE (6.31). Una posible elección para $g^{\perp}(x)$ es:

$$
g^{\perp}(x)=\left[\begin{array}{lll}
1 & 0 & 0 \\
0 & 0 & 1
\end{array}\right]
$$

la cual cumple con las condiciones para ser un aniquilador a izquierda de $g(x)$.

Para una clara interpretación de la PDE se representa la inversa de la matriz $Q(x)$, como:

$$
Q(x)^{-1}=\left[\begin{array}{ccc}
\frac{1}{q_{11}} & 0 & 0 \\
0 & \frac{1}{q_{22}} & 0 \\
0 & -\frac{q_{32}}{q_{22} q_{33}} & \frac{1}{q_{33}}
\end{array}\right]=\left[\begin{array}{ccc}
p_{11} & 0 & 0 \\
0 & p_{22} & 0 \\
0 & p_{32} & p_{33}
\end{array}\right]
$$

La ecuación en derivadas parciales (6.31) para esta aplicación es:

$$
g^{\perp}(x) Q^{-1}(x) \nabla P_{a}(x)=\left[\begin{array}{ccc}
1 & 0 & 0 \\
0 & 0 & 1
\end{array}\right]\left[\begin{array}{ccc}
p_{11} & 0 & 0 \\
0 & p_{22} & 0 \\
0 & p_{32} & p_{33}
\end{array}\right]\left[\begin{array}{c}
\frac{\partial P_{a}(x)}{\partial x_{1}} \\
\frac{\partial P_{a}(x)}{\partial x_{2}} \\
\frac{\partial P_{a}(x)}{\partial x_{3}}
\end{array}\right]=\left[\begin{array}{l}
0 \\
0
\end{array}\right] \Rightarrow
$$




$$
g^{\perp}(x) Q^{-1}(x) \nabla P_{a}(x)=\left\{\begin{array}{cc}
p_{11} \frac{\partial P_{a}(x)}{\partial x_{1}} & =0 \\
p_{32} \frac{\partial P_{a}}{\partial x_{2}}+p_{33} \frac{\partial P_{a}(x)}{\partial x_{3}} & =0
\end{array}\right.
$$

La solución de la PDE para este problema da como resultado una función $\Psi_{p}\left(x_{2}, x_{3}\right)$, dada por:

$$
\Psi_{p}\left(x_{2}, x_{3}\right)=\left(-\frac{k_{2}}{k_{1}} x_{2}+x_{3}\right)
$$

con $\Psi_{p}: \mathbb{R}^{2} \rightarrow \mathbb{R}$, de forma tal que $P(x)+P_{a}(x)$ tenga un mínimo en $x^{*}$.

La función $P_{a}(x)$ propuesta está dada por:

$$
P_{a}(x)=-\frac{R_{r} x_{1}^{*} x_{1}}{2 L_{r}}+k_{a}\left(z-z^{*}\right)^{2}+k_{b}\left(z-z^{*}\right),
$$

donde:

$$
\begin{aligned}
& z=-\frac{k_{2}}{k_{1}} x_{2}+x_{3}, \\
& z^{*}=-\frac{k_{2}}{k_{1}} x_{2}^{*}+x_{3}^{*}, \\
& k_{b}=\frac{R_{r} q_{32}}{L_{r}} x_{2}^{*}, \\
& k_{a}=1, \\
& k_{1}=\frac{-q_{32}}{q_{22} q_{33}}, \\
& k_{2}=\frac{1}{q_{33}} .
\end{aligned}
$$

La función de potencial mixto $P(x)$ para este problema es:

$$
P(x)=\int[Q(x) f(x)]^{T} d x=\frac{T_{m} x_{3}}{J}+\frac{B_{r} x_{3}}{2 J}+\frac{R_{r} x_{1}^{2}}{2 L_{r}}+\frac{3 U^{2} x_{2}^{2} L_{m}^{2} L_{r}}{2 J^{2} R_{r} \omega^{2} L_{s}^{2}} .
$$

El gradiente de la función $P_{d}(x)$, evaluada en el punto de equilibrio, para el controlador de potencia activa es:

$$
\left.\nabla P_{d}(x)\right|_{x=x^{*}}=\left[\begin{array}{c}
\frac{R_{r}}{2 L_{r}} x_{1}-\frac{R_{r}}{2 L_{r}} x_{1}^{*} \\
\frac{3 x_{2} L_{r}}{R_{r}}\left(\frac{U L_{m}}{J \omega L_{s}}\right)^{2}+\left(-k_{b} \frac{k_{2}}{k_{1}}\right)-2 k_{a} \frac{k_{2}}{k_{1}}\left(z-z^{*}\right) \\
\frac{T_{m} x_{3}}{J}+\frac{B_{r} x_{3}}{J}+k_{b}+2 k_{a}\left(z-z^{*}\right)
\end{array}\right]=0_{3 x 1} .
$$

El primer término de la ecuación (6.38) puede ser considerado como un valor de compensación sobre la familia de curvas definidas por la solución de la PDE (6.37). Por otra parte, la segunda fila de (6.40) debe verificar:

$$
\frac{3 x_{2} L_{r}}{R_{r}}\left(\frac{U L_{m}}{J \omega L_{s}}\right)^{2}+\left(-k_{b} \frac{k_{2}}{k_{1}}\right)-2 k_{a} \frac{k_{2}}{k_{1}}\left(z-z^{*}\right)=0 .
$$

Luego, la correcta elección de $k_{b}, k_{1}$ y $k_{2}$, permite que el gradiente sea nulo. Evaluando el gradiente, el último término de (6.41) es cero. Mientras que cuando se evalúa el Hessiano, esta expresión asegura que sea definido positivo.

Por último, el gradiente de $P_{d}$, con respecto a la variable $x_{3}$ debe cumplir con:

$$
\frac{T_{m} x_{3}}{J}+\frac{B_{r} x_{3}}{2 J}+k_{b}+2 k_{a}\left(z-z^{*}\right)=0
$$


En la expresión anterior, el último término $\left(z-z^{*}\right)$ es cero en el punto de equilibrio, además es necesario buscar una condición para que $k_{b}$ anule los dos primeros términos. Entonces, teniendo en cuenta que el par electromagnético está dado por:

$$
T_{e}=\frac{3 U L_{m}}{\omega L_{s}} x_{2}
$$

y observando que los dos primeros términos de (6.42) pueden representarse como:

$$
\frac{T_{e}^{*}}{J}=\left(\frac{T_{m}}{J}+\frac{B_{r} x_{3}}{J}\right)
$$

entonces,

$$
\frac{T_{e}^{*}}{J}=k_{b} \rightarrow k_{b}=q_{33} \frac{T_{e}}{J}
$$

El Hessiano para el controlador de potencia activa es:

$$
\nabla^{2} P_{d}(x)=\left[\begin{array}{ccc}
\frac{R_{r}}{L_{r}} & 0 & 0 \\
0 & t h_{1} & t h_{3} \\
0 & t h_{3} & t h_{2}
\end{array}\right]>0,
$$

donde

$$
\begin{gathered}
t h_{1}=2\left(\frac{k_{2}}{k_{1}}\right)^{2} k_{a}+\frac{3\left(U L_{m}\right)^{2} L_{r}}{R_{r}\left(J \omega L_{s}\right)^{2}}, \\
t h_{2}=\frac{B_{r}}{J}+2 k_{a}, \\
t h_{3}=-2 k_{a} \frac{k_{2}}{k_{1}} .
\end{gathered}
$$

Los menores principales de la expresión (6.44) están dados por:

$$
\begin{array}{r}
A_{1}=\frac{R_{r}}{L_{r}}>0, \\
A_{2}=\frac{R_{r}}{L_{r}} t_{h 1}>0, \\
A_{3}=\frac{R_{r}}{L_{r}}\left[t_{h 1} t_{h 2}-t_{h 3}^{2}\right]>0 .
\end{array}
$$

Los valores $A_{1}, A_{2}, A_{3}$ son valores positivos, de esta forma se verifica que la función $P_{d}(x)$ tiene un mínimo en el punto de equilibrio $x^{*}$.

\section{Cálculo de la acción de control para la potencia activa}

Partiendo de la expresión enunciada en el Capítulo 5:

$$
u=\left[g^{T} Q^{T} Q g\right]^{-1} g^{T} Q^{T} \nabla P_{a} .
$$

Entonces, la acción de control para la potencia activa está dada por:

$$
u_{P}=\left(\frac{L_{r} q_{22}}{q_{22}^{2}+q_{32}^{2}}\right) \frac{\partial P_{a}(x)}{\partial x_{2}}+\left(\frac{L_{r} q_{32}}{q_{22}^{2}+q_{32}^{2}}\right) \frac{\partial P_{a}(x)}{\partial x_{3}} .
$$




\section{Análisis del controlador de potencia activa}

En esta subsección el controlador desarrollado en la sección previa es evaluado. En la Figura 6.2 se muestra un aerogenerador de una potencia de 1,5 MW conectado a un sistema de distribución eléctrica de $25 \mathrm{kV}$. El cual exporta potencia a una red de $120 \mathrm{kV}$ a través de una línea de $30 \mathrm{~km}$ (Miller et al., 2003). Esta arquitectura simple, que ya ha sido utilizada en diversos trabajos facilita la evaluación del comportamiento del control propuesto (Evangelista et al., 2012; Fernández et al., 2010).

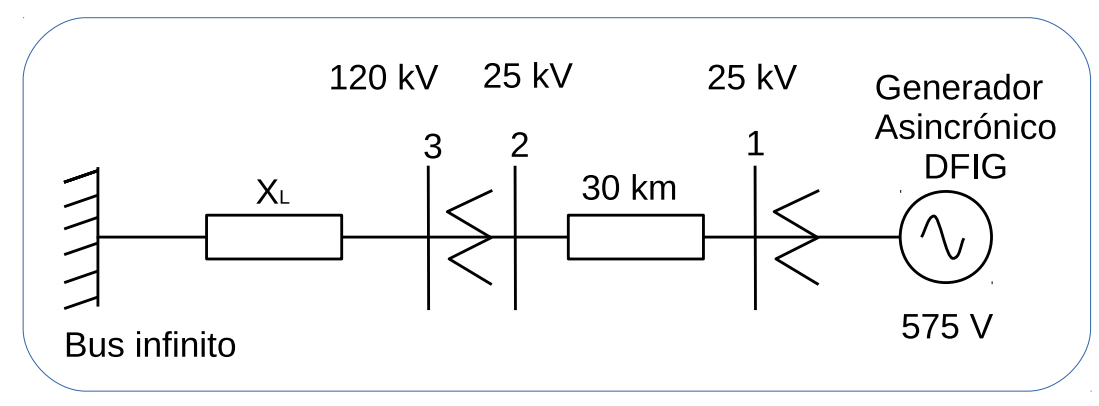

Figura 6.2: Esquema del sistema considerado.

Cuando la velocidad del viento es menor a la velocidad nominal, el ángulo de la pala del aerogenerador es mínimo con el objetivo de maximizar la potencia capturada por la turbina eólica. En régimen de viento superior al nominal se adapta el ángulo de paso para capturar la potencia nominal de forma controlada hasta la velocidad de corte.

En la zonas de vientos inferiores al nominal, la contribución de potencia activa inyectada a la red de distribución se puede llevar a cabo controlando la velocidad de giro del generador a partir de la siguiente expresión para la velocidad de referencia del eje del aerogenerador (Miller et al., 2003):

$$
\Omega_{\text {ref }}=-0,67 P_{t}^{2}+1,42 P_{t}+0,51,
$$

siendo $P_{t}$ la potencia extraída del viento. La curva de seguimiento de potencia (6.50) se muestra en la Figura 6.3, donde también se presentan las curvas características de potencia en función de diferentes velocidades de viento.

La regulación de la tensión en el punto de conexión del aerogenerador es realizada controlando la potencia reactiva que éste suministra a la red de la Figura 6.2. En este sentido, el regulador de tensión en el caso de que exista un apartamiento de la tensión de referencia puede contribuir tratando de estabilizar la tensión inyectando potencia reactiva. En este caso, donde el control por moldeo de potencia solo se aplica a la potencia activa, un controlador PI es utilizado para el control de la potencia reactiva, el cual es desarrollado en (Miller et al., 2003).

Dos casos son examinados para analizar la operación del sistema de control, una variación en el recurso eólico y una caída de tensión en el sistema de $120 \mathrm{kV}$.

El primer caso considerado es un cambio abrupto en la velocidad del viento. Aunque, un escalón en la velocidad del viento resulta una situación que no se pondrá de manifiesto en el funcionamiento habitual del aerogenerador resulta de utilidad para estudiar el comportamiento del sistema ante una exigencia importante. Debido al contenido armónico, las variaciones debidas a escalones son mucho más demandantes que perfiles reales de variación del viento. En este sentido, en la Figura 6.4 a) se observa un escalón en la velocidad del viento 


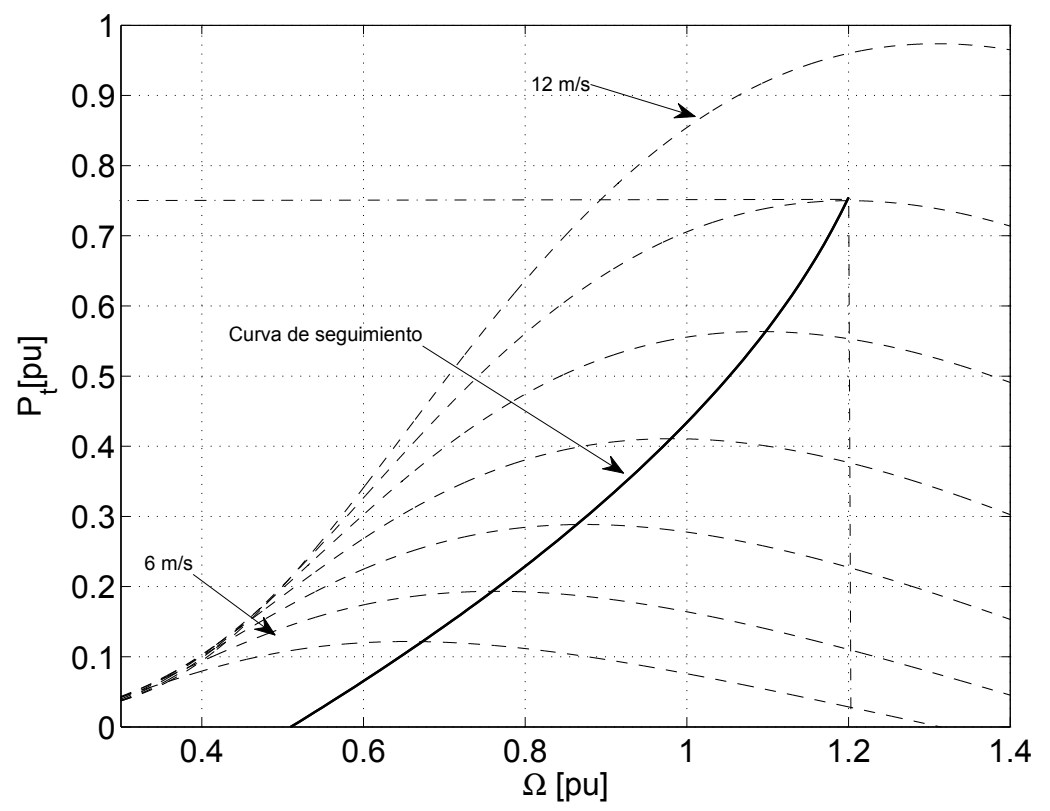

Figura 6.3: Curva de seguimiento del aerogenerador.

que implica un cambio en el par de la turbina eólica que da lugar a un par acelerador que incrementa la velocidad del eje del generador, Figura 6.4 c). Como consecuencia del aumento de velocidad de la turbina eólica, la potencia extraída del aerogenerador se incrementa como se observa en la Figura 6.4 b). Entonces, la estrategia de control propuesta asegura que la característica transitoria evolucione hacia el punto de funcionamiento de máxima eficiencia de $10 \mathrm{~m} / \mathrm{s}$. La velocidad de la variación de potencia, relativamente lenta es debida a que la referencia para el lazo de potencia es una curva de seguimiento de la potencia mecánica que depende de la inercia de la turbina. Esta potencia no varía hasta que se produce un cambio en la velocidad del eje del rotor producto de la variación en la velocidad del viento.

El segundo caso considerado es un hueco de tensión en el sistema de potencia de $120 \mathrm{kV}$ de la Figura 6.2. La tensión cae a 0,6 p.u. y tiene una duración de 0,6 segundos, como se muestra en línea continua en la Figura 6.5 a). Con línea de trazos se observa la tensión en el punto de conexión del aerogenerador. Como se observa en la Figura 6.5 b) una fuerte inyección de potencia activa es provista por el DFIG para prevenir que la velocidad del eje se incremente. Este hecho puede ser verificado en la Figura $6.5 \mathrm{c}$ ), donde no se observan variaciones que provoquen que el aerogenerador se desconecte de la red de distribución. En $t>10,6$ segundos la falla es superada y el sistema evoluciona a un valor de estado estacionario. 

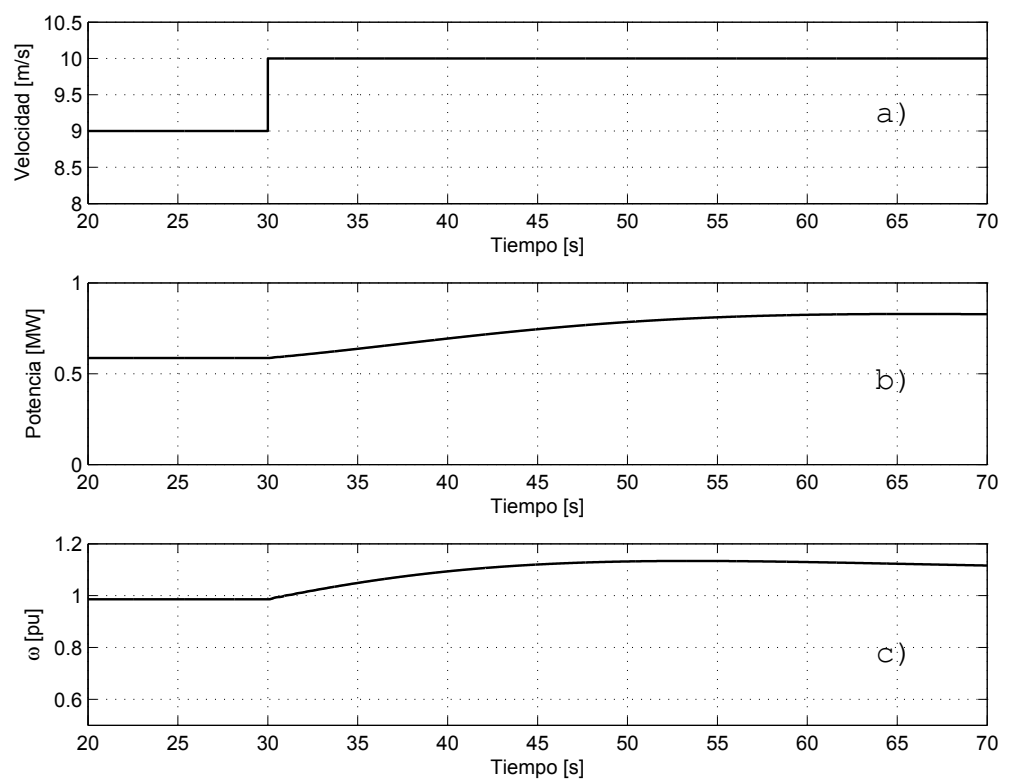

Figura 6.4: Comportamiento del sistema analizado ante un escalón de viento. a) Escalón de viento.

b) Potencia activa. c) Velocidad del eje del aerogenerador.
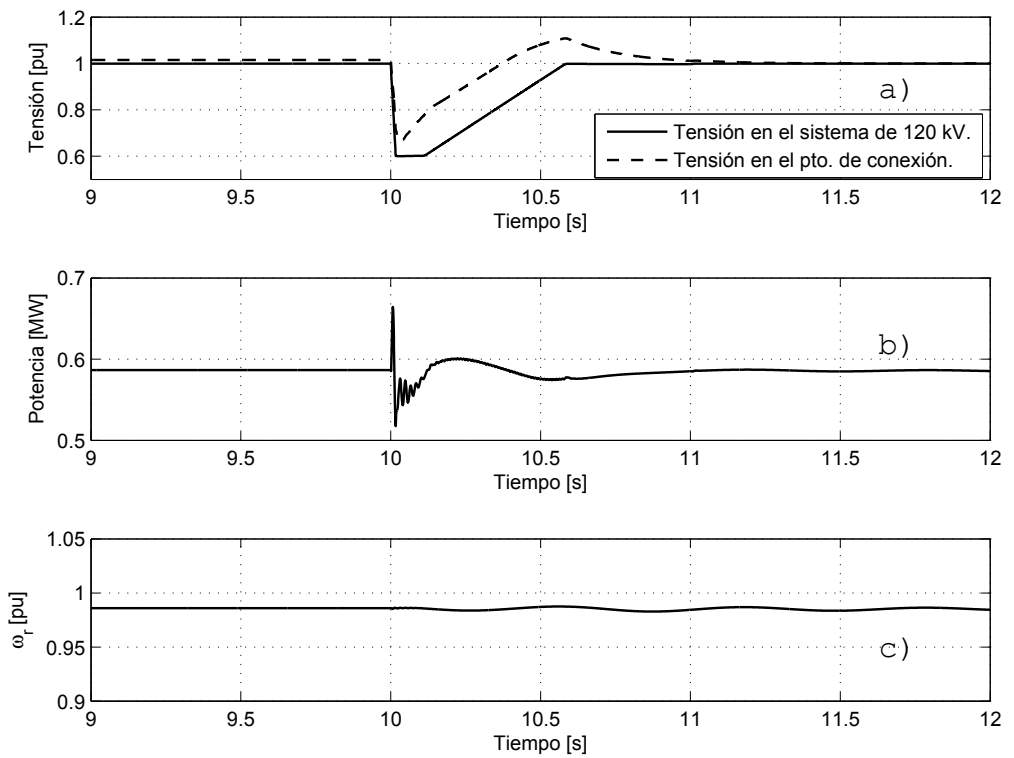

Figura 6.5: Hueco de tensión. a) Tensión sobre el sistema de $120 \mathrm{kV}$ y el punto de conexión. b) Potencia activa. c) Velocidad del eje del aerogenerador.

\subsubsection{Controladores de potencias activa y reactiva con acción integral}

Aprovechando la elección de un marco de referencia de flujo orientado según la tensión de red, se pueden diseñar dos controladores independientes que logran controlar las potencias activa y reactiva que aporta un generador asincrónico de rotor bobinado. Empleando el 
modelo de la ecuación (6.12) $g(x)$ es dividido en:

$$
g(x)=\left[\begin{array}{ll}
g_{a}(x) & g_{r}(x)
\end{array}\right]
$$

con

$$
g_{a}(x)=\left[\begin{array}{c}
0 \\
1 / L_{r} \\
0
\end{array}\right], g_{r}(x)=\left[\begin{array}{c}
1 / L_{r} \\
0 \\
0
\end{array}\right]
$$

donde $g_{a}(x)$ y $g_{r}(x)$ son las submatrices de $g(x)$ para el control de la potencias activa y reactiva, respectivamente.

Se proponen dos funciones para el cálculo de los controladores, una para cada potencia, que deben cumplir simultáneamente con $x^{*}=\arg \min P_{d}(x)$, con $P_{d}(x)=P(x)+P_{a}(x)$. Luego, la función $P_{a}(x)$ está dada por:

$$
P_{a}(x)=P_{a P}(x)+P_{a Q}(x),
$$

donde $P_{a P}$ y $P_{a Q}$ son las funciones que moldean la potencia activa y reactiva, respectivamente. Dado que las acciones de control están desacopladas, para la potencia activa se propone la siguiente función:

$$
P_{a P}(x)=k_{a}\left(z-z^{*}\right)^{2}+k_{b}\left(z-z^{*}\right) .
$$

Para proponer una función que balancee la potencia reactiva, es necesario resolver la PDE (6.31). Para ello se elige el aniquilador a izquierda $\left(g_{Q}^{\perp}(x)\right)$ de $g_{r}(x)$ como:

$$
g_{Q}^{\perp}(x)=\left[\begin{array}{lll}
0 & 1 & 0 \\
0 & 0 & 1
\end{array}\right] .
$$

Luego:

$$
g_{Q}^{\perp} Q^{-1} \nabla P_{a Q}=\left[\begin{array}{ccc}
0 & p_{22} & 0 \\
0 & p_{32} & p_{33}
\end{array}\right] \nabla P_{a Q}=0 .
$$

Las ecuaciones en derivadas parciales son:

$$
g_{Q}^{\perp} Q^{-1} \nabla P_{a Q}=\left\{\begin{array}{cl}
p_{22}\left(\frac{\partial P_{a Q}(x)}{\partial x_{2}}\right) & =0, \\
p_{32}\left(\frac{\partial P_{a Q}(x)}{\partial x_{2}}\right)+p_{33}\left(\frac{\partial P_{a Q}(x)}{\partial x_{3}}\right) & =0 .
\end{array}\right.
$$

Entonces, la $P_{a Q}(x)=\Psi_{Q}\left(x_{1}\right)$ propuesta es:

$$
P_{a Q}(x)=\left(z_{1}-z_{1}^{*}\right)+\left(z_{1}-z_{1}^{*}\right)^{2},
$$

con:

$$
\begin{aligned}
& z_{1}=c_{2} x_{1}, \\
& z_{1}^{*}=c_{2} x_{1}^{*}, \\
& c_{2}=\frac{q_{11} R_{r}}{2 L_{r}} .
\end{aligned}
$$


Debido a que se tienen dos controladores uno de potencia activa y otro de potencia reactiva, el gradiente de la función potencial total está dado por:

$$
\left.\nabla P_{d}(x)\right|_{x=x^{*}}=\left[\begin{array}{c}
\frac{R_{r}}{2 L_{r}} x_{1}+q_{11} \frac{R_{r}}{L_{r}} x_{1}+2 c_{2}\left(z_{1}-z_{1}^{*}\right) \\
\frac{3 x_{2} L_{r}}{R_{r}}\left(\frac{U L_{m}}{J \omega L_{s}}\right)^{2}+\left(-k_{b} \frac{k_{2}}{k_{1}}\right)+2 k_{a}\left(z-z^{*}\right) \\
\frac{T_{m} x_{3}}{J}+\frac{B_{r} x_{3}}{J}+k_{b}+2 k_{a}\left(z-z^{*}\right)
\end{array}\right]=0_{3 x 1} .
$$

Como $q_{11}=-1$, y como $\left(z_{1}-z_{1}^{*}\right)=0$ en la primera fila de la última expresión (6.60), cumple con la condición del gradiente. Las siguientes filas de la expresión del gradiente no sufren modificaciones con respecto a la ecuación (6.40).

El Hessiano para el sistema con el controlador de potencia activa y de potencia reactiva queda definido por la expresión (6.44). De esta forma se cumplen con las condiciones del gradiente y del Hessiano.

\section{Cálculo de las acciones de control}

Las acciones de control son calculadas empleando la expresión (6.48). Entonces para el controlador de potencia activa del generador asincrónico de rotor bobinado la acción de control resulta (Peña et al., 2015):

$$
u_{P}=\left(\frac{L_{r} q_{22}}{q_{22}^{2}+q_{32}^{2}}\right) \frac{\partial P_{a P}(x)}{\partial x_{2}}+\left(\frac{L_{r} q_{32}}{q_{22}^{2}+q_{32}^{2}}\right) \frac{\partial P_{a P}(x)}{\partial x_{3}},
$$

y la acción de control para la potencia reactiva está dada por (Peña et al., 2015):

$$
u_{Q}=\frac{L_{r}}{q_{11}} \frac{\partial P_{a Q}(x)}{\partial x_{1}},
$$

siendo $q_{11}, q_{22}$ y $q_{32}$ los elementos de la matriz $Q(x)$ presentada en la ecuación (6.24), $P_{a P}(x)$ y $P_{a Q}(x)$ son las funciones propuestas (6.54) y (6.58).

Como se comentó en el capítulo anterior, un controlador con acción integral tiene la capacidad de compensar errores de estado estacionario causados por perturbaciones de baja frecuencia o incertezas en el modelo. De esta forma, agregando una acción de control integral sobre la señal de error a ser regulada se logra un diseño robusto del controlador (Romero et al., 2013). En este sentido, asumiendo una perturbación constante en la entrada es posible proponer un control por moldeo de potencia con acción integral en lazo cerrado que conserva la estructura de las expresiones de Brayton-Moser. En este caso las ecuaciones de BraytonMoser pueden ser escritas como se mencionó en el Capítulo 5:

$$
Q(x) \dot{x}=\frac{\partial P}{\partial x}+g(x)(u-d),
$$

donde $d$ es una perturbación constante, la acción de control está dada por la acción de control calculada mediante moldeo de potencia $\left(u_{P S}\right)$ más la acción integral $(\tau)$ :

$$
u=u_{P S}+\tau \text {. }
$$

siendo $\tau$ el estado integral con la dinámica:

$$
\dot{\tau}=-k_{i} g^{T}(x) Q(x)^{-1} \frac{\partial P_{d}(x)}{\partial x},
$$


con $k_{i}>0$.

Si la señal error resulta ser una salida pasiva la estabilidad se conserva a pesar de la adición de una acción integral (Romero et al., 2013).

Las acciones de control para los controladores de la potencia activa y la potencia reactiva para el DFIG, evaluando la expresión (6.63) son:

$$
u_{F P}=\left(\frac{L_{r} q_{22}}{q_{22}^{2}+q_{32}^{2}}\right) \frac{\partial P_{a P}(x)}{\partial x_{2}}+\left(\frac{L_{r} q_{32}}{q_{22}^{2}+q_{32}^{2}}\right) \frac{\partial P_{a P}(x)}{\partial x_{3}}+\tau_{a},
$$

y

$$
u_{F Q}=\left(\frac{L_{r}}{q_{11}}\right) \frac{\partial P_{a Q}(x)}{\partial x_{1}}+\tau_{r},
$$

donde las acciones integrales $\tau_{a}$ y $\tau_{r}$ son obtenidas a partir de la ecuación (5.82), siendo sus dinámicas:

$$
\dot{\tau_{a}}=\left(-\frac{k_{i a}}{L_{r} q_{22}}\right) \frac{\partial P_{d}(x)}{\partial x_{2}},
$$

y

$$
\dot{\tau_{r}}=\left(-\frac{k_{i r}}{q_{11} L_{r}}\right) \frac{\partial P_{d}(x)}{\partial x_{1}},
$$

con $P_{d}(x)$ dada por la ecuación $(6.30), k_{i a}>0$ y $k_{i r}>0$.

\subsubsection{Control de una granja eólica}

Consideremos ahora otro trabajo, desarrollado en el marco de la tesis en el que se analizó una granja eólica con seis aerogeneradores de 1,5 MW cada uno. Es bien conocido que, las expresiones que capturan la dinámica de los aerogeneradores son complejas y por lo tanto el costo computacional para la simulación de un parque eólico empleando modelos detallados es alto. Para analizar un sistema de estas características es necesario desarrollar modelos equivalentes de las granjas eólicas. Entonces, con el objetivo de evaluar la técnica de control por moldeo de potencia en un grupo de seis aerogeneradores se emplean los conceptos relacionados a un aerogenerador equivalente o modelo agregado desarrollado en Slootweg y Kling (2003); Matevosyan et al. (2011) para reducir el tiempo de simulación. Para representar el funcionamiento de los seis aerogeneradores son utilizados los conceptos referidos a un aerogenerador equivalente. La potencia nominal del aerogenerador equivalente es de $9 \mathrm{MW}$. Los aerogeneradores aportan potencia al punto de conexión de la misma forma que en el caso del esquema de la Figura 6.2.

Para este caso, se plantea una referencia de par para el control indirecto de la potencia activa. Esta es obtenida a partir de la curva de seguimiento del óptimo de la siguiente forma:

$$
T_{r e f}\left(\omega_{r}\right)=\frac{P_{r e f}\left(\omega_{r}\right)}{\omega_{r}},
$$

la cual permite extraer la máxima potencia del viento. $P_{r e f}$ es la potencia de referencia, la cual puede ser obtenida mediante el seguimiento de la potencia óptima o a partir de resolver la expresión (6.50) (Miller et al., 2003).

Además, considerando que el sistema eólico debe contribuir a la calidad de la energía eléctrica suministrada a la carga, dos estrategias para el control de la potencia reactiva en 
los aerogeneradores son analizadas. La primera estrategia fue planteada con la finalidad de que toda la potencia reactiva que consume la granja sea provista mediante el control de los convertidores conectados a los rotores de los generadores DFIG. Es decir, el grupo de aerogeneradores es visto, desde el punto de vista de la potencia reactiva como neutral a la red. La segunda estrategia fue diseñada con el objetivo de aportar potencia reactiva para tratar de contribuir a la regulación de la tensión en el punto de conexión de la granja.

En este sentido podemos resumir estos objetivos de la siguiente forma:

- Estrategia 1, control de la potencia activa acorde a la ecuación (6.69) y control de la potencia reactiva neutral a la red, es decir $Q_{r e f}=0$.

- Estrategia 2, control de la potencia activa a través de (6.69) y el control de la potencia reactiva (regulación de tensión), entonces $Q_{r e f}=f(U)$.

A partir de las expresiones de la corriente en el estator con el eje "y" del marco de referencia alineado con la tensión de red, la corriente $i_{r x}$ puede ser expresada como:

$$
i_{r x}=\frac{U^{2}-(2 / 3) Q_{s} \omega L_{s}}{\omega L_{m} U} .
$$

Entonces, la referencia de corriente para $Q_{r e f}=0$ es:

$$
i_{\text {rxref }}=\frac{U}{\omega L_{m}} .
$$

Mientras que para la segunda estrategia, el control de $Q$ para regulación de tensión, se propone la siguiente corriente de referencia:

$$
i_{r x r e f}=\left(\frac{U_{r e f}-U}{L_{m} \omega}\right) k_{1}+k_{2},
$$

donde $U_{\text {ref }}$ es la tensión de referencia ( $1 \mathrm{pu}$ ), $k_{1}$ es una constante de ajuste y $k_{2}$ asegura que la potencia reactiva inyectada por el generador es cero cuando la tensión en el punto de conexión es la tensión nominal. La ley de control proporcional (6.72) implica que una corriente de referencia $\left(i_{\text {rxref }}\right)$ intenta restaurar la tensión en el punto de conexión, incrementando la inyección de potencia reactiva por parte de los DFIG.

Un esquema del sistema de control puede observarse en la Figura 6.6. En la misma se ponen de manifiesto los dos controladores por moldeo de potencia y además se puede observar las dos estrategias elaboradas, $\left(Q_{r e f}=0\right.$ y $\left.Q_{r e f}=f(U)\right)$, para el control de la potencia reactiva que entrega el aerogenerador.

\section{Resultados de simulación}

Tres casos de prueba fueron evaluados para comprobar la validez de los controladores de potencias activa y reactiva en (Peña et al., 2015). El primer caso analizado fue una variación en el recurso eólico como la que se presentó en la Figura 6.4. La Figura 6.7 considera este primer caso, empleando la estrategia reactivo neutro, para la granja eólica. En la parte a) de esta figura se puede observar la variación en la velocidad del viento la cual induce una variación de par mecánico (Figura 6.7 c)) que incrementa la velocidad del eje del generador 


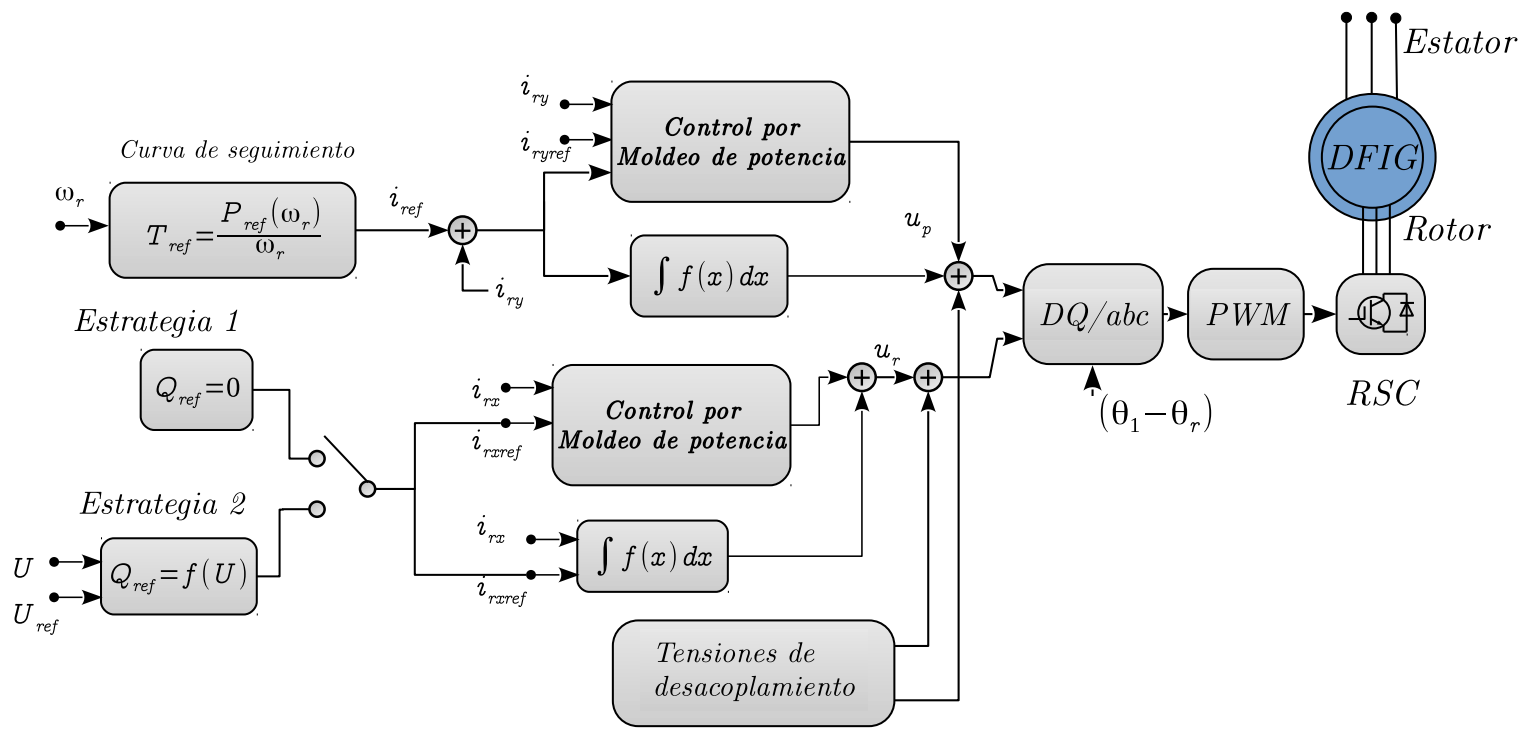

Figura 6.6: Esquema de la implementación de la técnica de control con acción integral para la granja eólica.

(Figura $6.7 \mathrm{~d}$ ) ). Finalmente, la potencia activa que aporta el aerogenerador a la red es mostrada en la Figura 6.7 c).

El controlador propuesto fuerza al sistema a seguir la referencia de par, según la ecuación (6.69), incrementando la potencia entregada a la red eléctrica dada por la velocidad de la turbina. Es importante notar que el par mecánico experimenta un cambio abrupto debido a la variación en el recurso eólico pero la potencia eléctrica generada sigue la referencia de la velocidad de giro. Tanto el par mecánico como el par eléctrico solo coinciden en estado estacionario. La contribución del término integral del controlador es mostrado en la Figura 6.8 b), frente a la perturbación de viento mostrada en la Figura 6.8 a). La salida del integrador varía para eliminar el efecto de la perturbación constante sobre la corriente (Figura 6.8 b)). El error en la corriente $\left(i_{r y}\right)$ se muestra en la Figura $6.8 \mathrm{c}$ ).

El segundo caso considerado es una caída de tensión simétrica en el sistema de potencia de $120 \mathrm{kV}$ de 0,4 pu y con una duración de 0,6 segundos. La consideración de este tipo de fallas es útil para evaluar el cumplimiento de los requerimientos para la conexión de parques eólicos a la red. Uno de los requisitos más importantes es la capacidad del sistema para manejar una falla sin salir de funcionamiento (FRT, por su sigla en inglés, Fault ride-through). Es decir, en el caso que exista una falla en la red eléctrica como por ejemplo una caída de tensión, los aerogeneradores deben contribuir con la red eléctrica intentando mitigar el problema (Piwko et al., 2010; Ezzat et al., 2013). En la Figura 6.9 a) se observa la tensión en el sistema de $120 \mathrm{kV}$ en línea continua y la tensión en el punto de conexión del parque eólico en tres situaciones: control reactivo neutral ("SC") en línea de trazos (ecuación (6.71)), control de la tensión propuesto en este trabajo de tesis ("CC") en línea continua (ecuación 6.72), y un controlador ("PI") desarrollado en (Miller et al., 2003) en línea de trazos y puntos.

El controlador indicado como ("PI") tiene como objetivo regular la tensión en el punto de conexión de la granja eólica actuando sobre la potencia reactiva que los aerogeneradores pueden entregar. Las corrientes entregadas por el parque eólico en los casos "SC" (línea de trazos), "CC" (línea continua) y "PI" (línea de trazos y punto) pueden ser observadas en la Figura 6.9 b). 

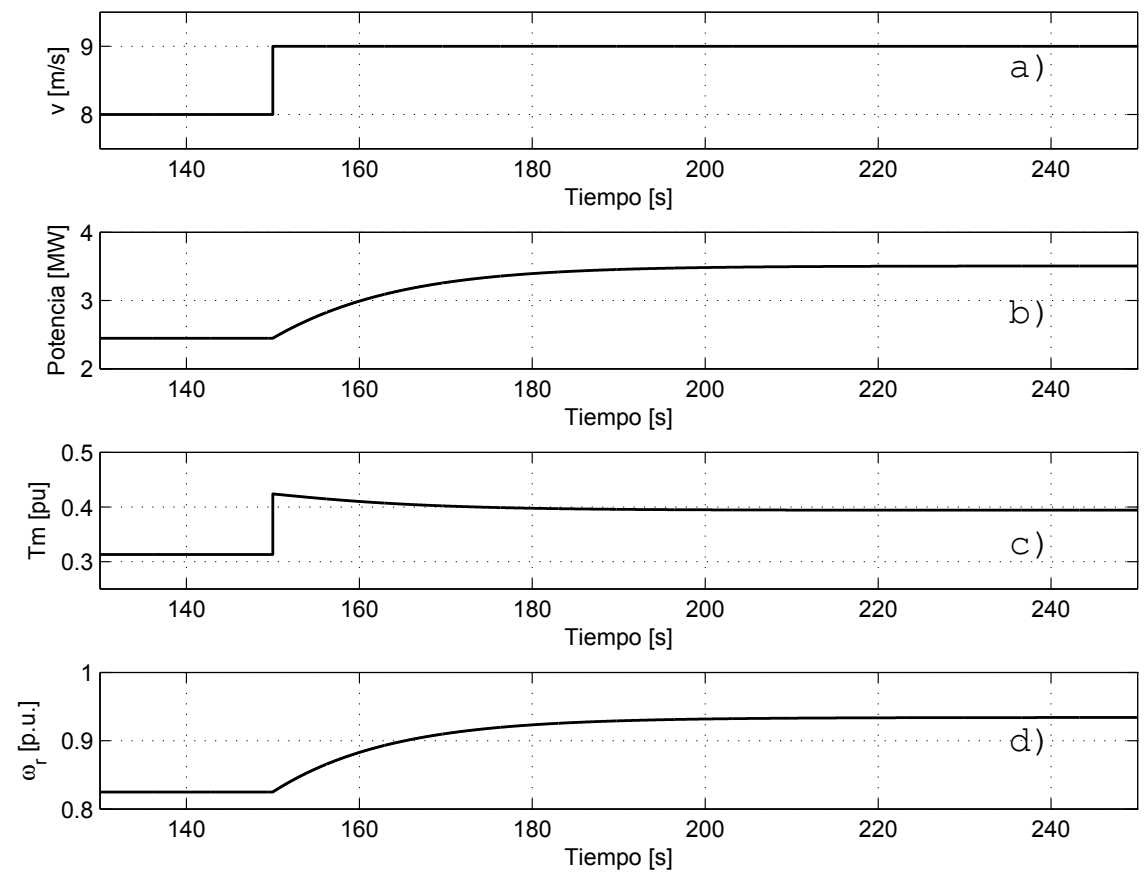

Figura 6.7: Sistema analizado frente a un escalón de viento. a) Escalón en la velocidad del viento. b) Potencia activa. c) Par mecánico. d) Velocidad en el eje.
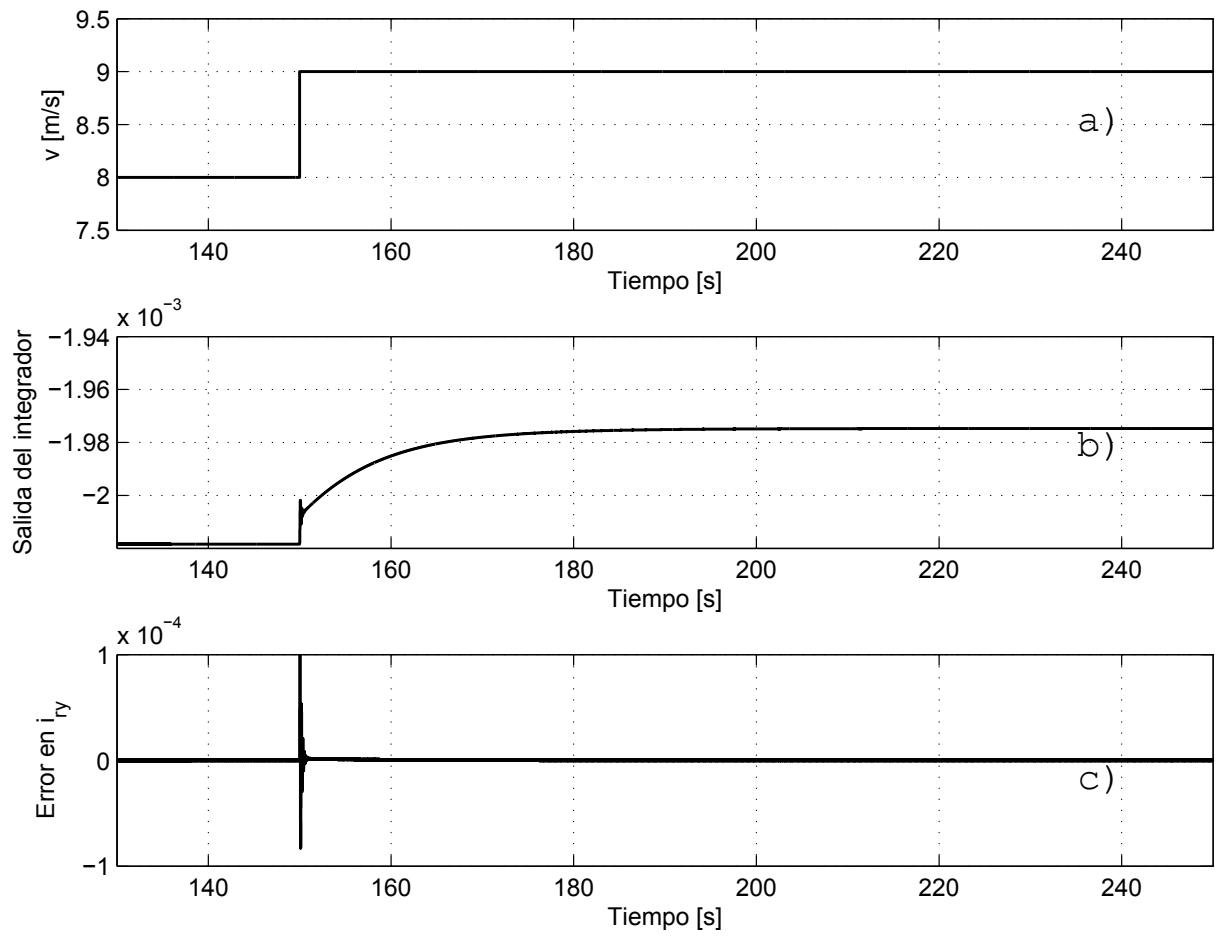

Figura 6.8: Sistema analizado frente a un escalón de viento. a) Escalón en la velocidad del viento. b) Acción integral. c) Error en $i_{r y} ;\left(x_{2}-x_{2}^{*}\right)$. 
Antes de que se produzca el hueco de tensión, el valor de estado estacionario de la corriente es tal que la potencia reactiva es nula (Figura $6.9 \mathrm{c}$ )). Cuando las estrategias "CC" y "PI" son utilizadas, luego de la falla, un incremento en la corriente es detectado (Figura 6.9 b)). Como consecuencia, mejora la tensión en el punto de conexión de la granja eólica (Figura 6.9 a)).

Comparando los resultados de las tres estrategias de control de potencia reactiva, se observa que la estrategia que es denominada neutral a la red, presenta un buen desempeño para condiciones normales aunque, esto no contribuye eficientemente para restaurar la tensión en el punto de conexión. Tanto la estrategia "PI" como la "CC" inyectan potencia reactiva a la red eléctrica intentando recuperar la tensión en el punto de conexión del parque eólico, como se observa en la Figura 6.9 a). En el inicio de la falla, debido a la inyección de potencia reactiva, Figura 6.9 c), la estrategia "PI" es más lenta que la estrategia "CC". Entonces, la caída de tensión en el punto de conexión inicialmente es más profunda en la estrategia "PI", la cual, también presenta un sobrepaso cuando el sistema se recupera de la falla Figura 6.9 a). Además, luego de que la falla es superada, la potencia reactiva en la estrategia "PI" lentamente recupera su valor en estado estacionario (Figura 6.9 c)). Una vez que el sistema se recupera de la falla, la tensión en el punto de conexión es restaurada (Figura 6.9 a) y Figura 6.10 a)).

La Figura 6.10 muestra el desempeño del controlador de potencia activa en presencia de la caída de tensión en el sistema de $120 \mathrm{kV}$. Por una cuestión de claridad, la Figura 6.9 a) es repetida en la Figura 6.10 a). En la Figura 6.10 b) puede observarse el aporte de la potencia activa, particularmente cómo la potencia es restaurada al valor de estado estacionario luego de que el sistema se restablece de la falla. En la Figura 6.10 c) una pequeña variación de la velocidad del eje en relación a la magnitud de la falla es observada. Nuevamente puede concluirse que la acción de control con la estrategia propuesta posee un valor máximo del orden del valor obtenido con la estrategia "PI".

El efecto de la acción integral sobre la variable $i_{r x}$ es mostrada en la Figura 6.11. La contribución de la acción integral es presentada en la Figura 6.11 b), la cual logra error de estado estacionario cero (Figura $6.11 \mathrm{c}$ )).

El tercer caso considerado es una caída de tensión desbalanceada de 0.4 p.u. y 0.6 segundos de duración en una de las fases empleando la estrategia "CC". Cuando el sistema esta balanceado, las corrientes en el DFIG solo son de secuencia positiva, si ocurre un desbalance hay una corriente de secuencia negativa (Martinez et al., 2012). En la Figura 6.12 a) se muestran las corrientes de secuencia positivas y negativas en línea continua y en línea de trazos, respectivamente. Las potencias activa y reactiva entregadas por el aerogenerador se presentan en la Figura 6.12 b) y en la Figura 6.12 c). Finalmente, en la Figura 6.12 d) se muestran las características del par electromagnético frente a una variación de tensión desbalanceada. Es conocido que la presencia de desbalances implican un comportamiento oscilatorio tanto del par del aerogenerador como de la tensión en el bus de continua cuando dicho desbalance no es tomado en cuenta en la estrategia de control. Luego, como trabajo futuro se establecerá un controlador que a partir de la separación de secuencias permita corregir los problemas asociados a las fallas desbalanceadas. 

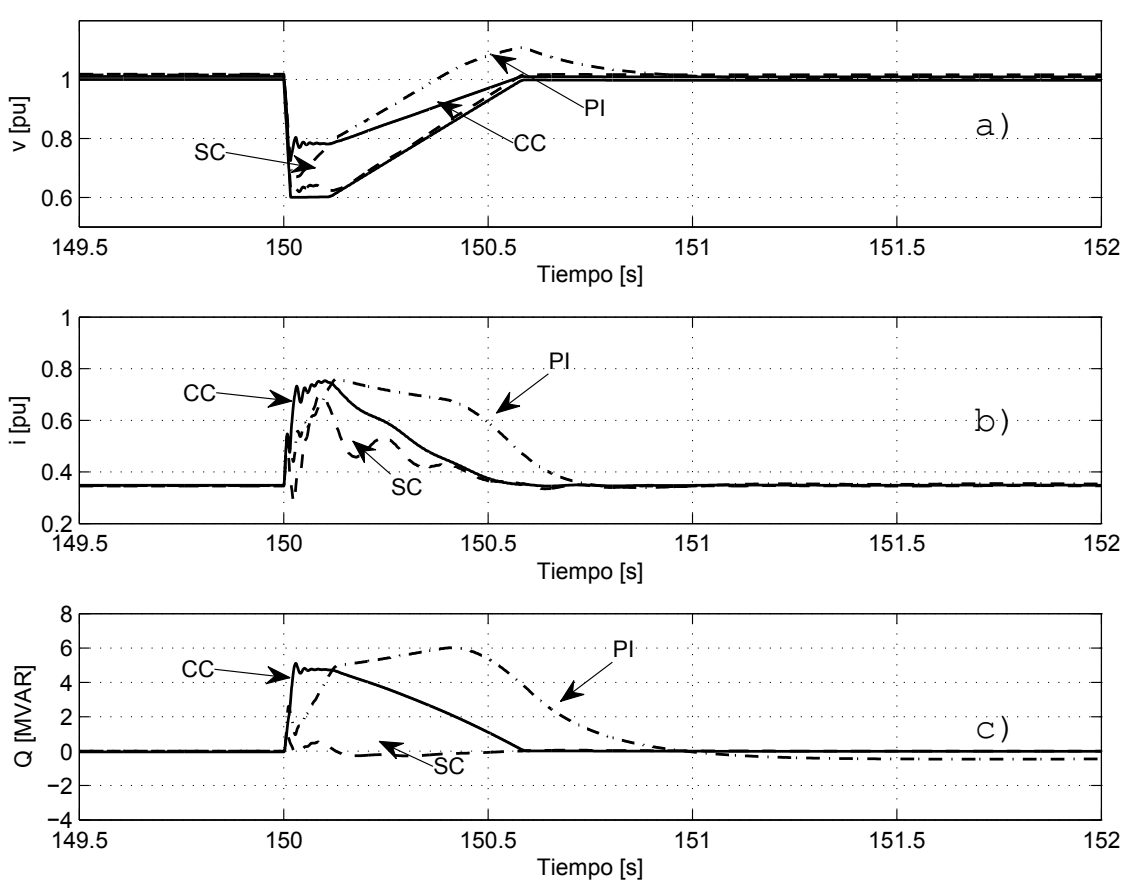

Figura 6.9: a) Tensión en el bus de $120 \mathrm{kV}$ (línea continua y tensión en el punto de conexión utilizando CC(línea continua), SC (línea de trazos) y una estrategia PI (línea de trazos y puntos). b) Corriente inyectada por la granja eólica c) Potencia reactiva en los tres casos analizados.
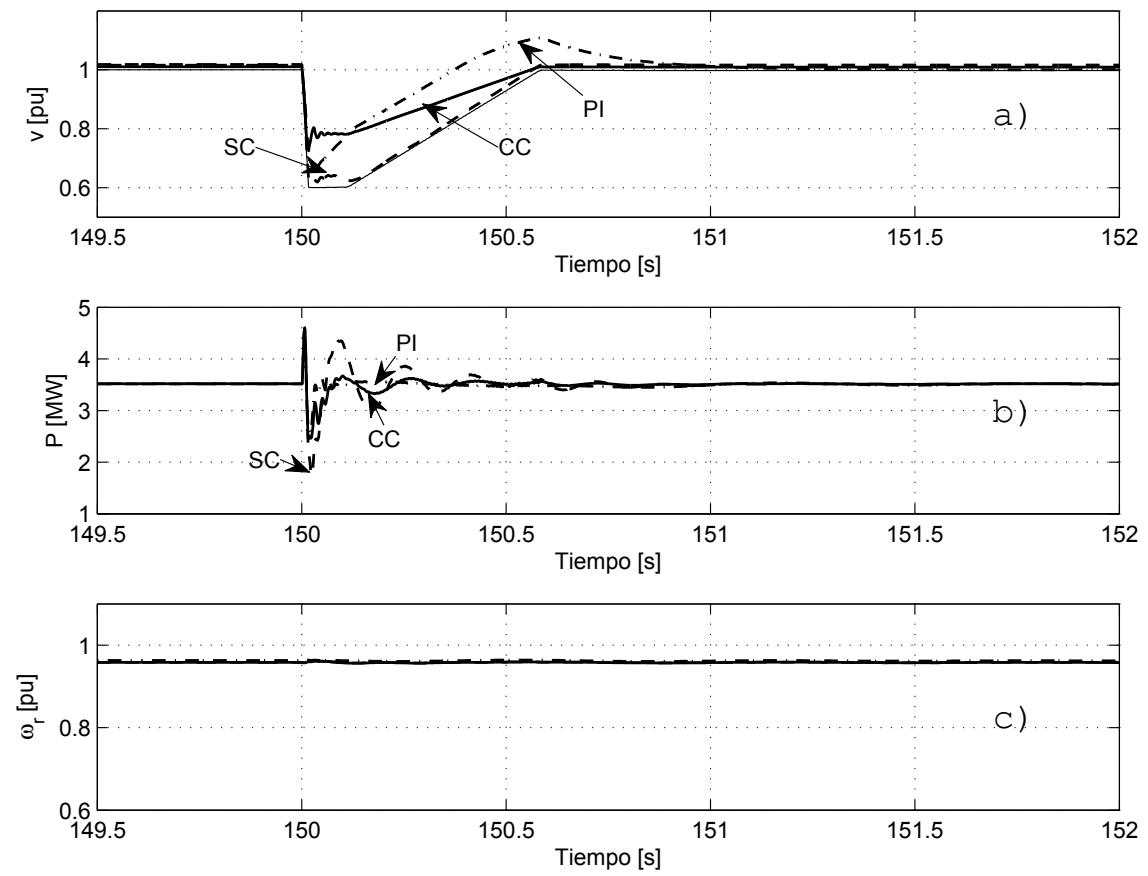

Figura 6.10: a) Tensión en el bus de $120 \mathrm{kV}$ (línea continua) y tensión en el punto de conexión para los tres casos analizados. b) Potencia activa. c) Velocidad del eje del rotor. 

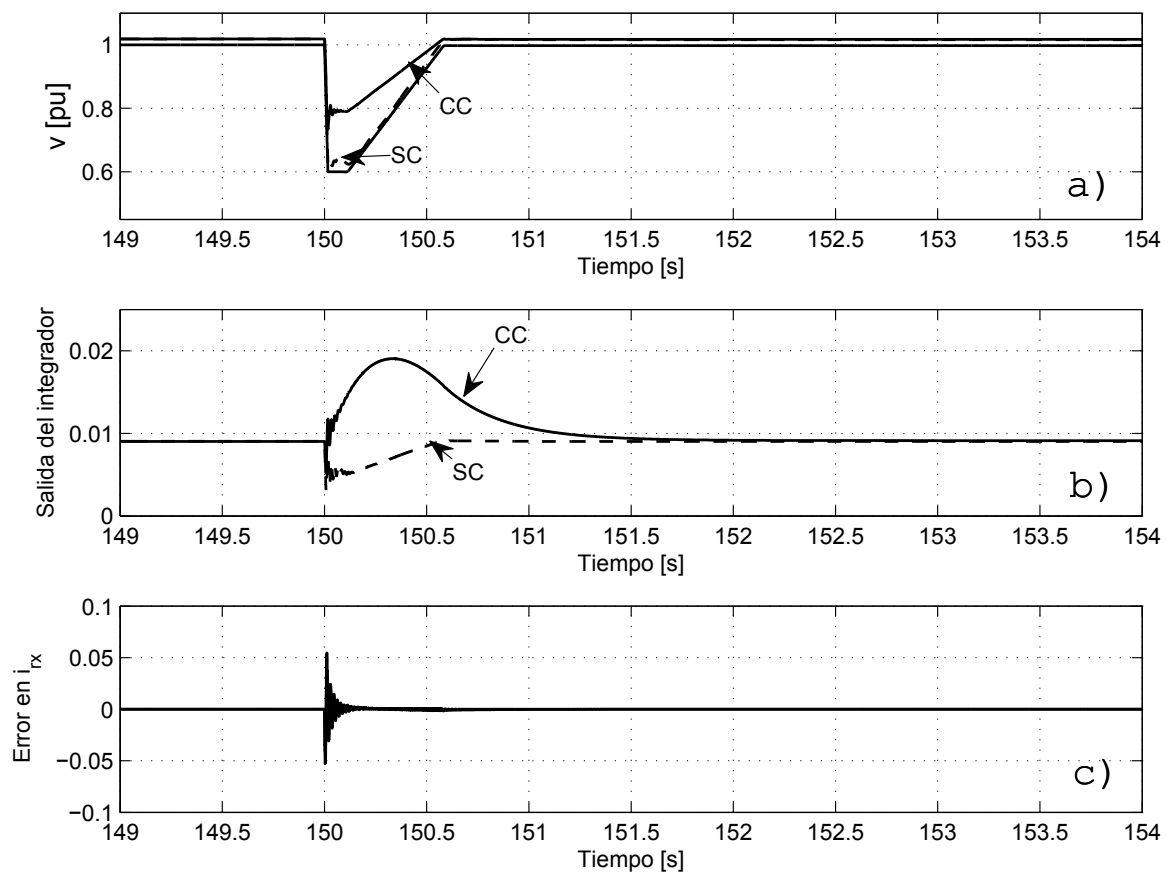

Figura 6.11: a) Tensión en el bus de $120 \mathrm{kV}$ (línea continua) y tensión en el punto de conexión para las dos estrategias analizadas. b) Salida del integrador. c) Error en $i_{r x} ;\left(x_{1}^{*}-x_{1}\right)$.
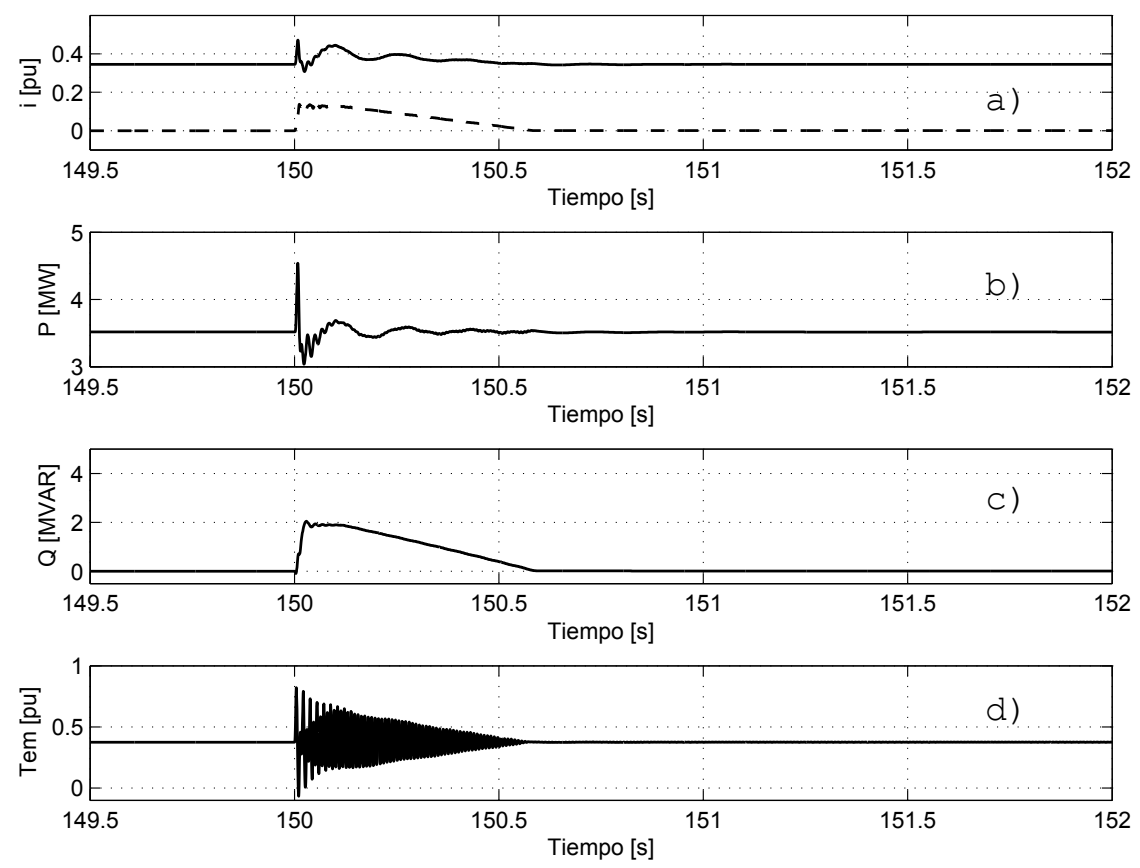

Figura 6.12: a) Corriente de secuencia positiva (línea continua) y corriente de secuencia negativa (línea de trazos) en el punto de conexión. b) Potencia activa. c) Potencia reactiva. d) Par electromagnético. 


\subsection{Aplicación a una micro red}

Como se indicó en el capítulo referido a redes eléctricas, existen diferentes definiciones de micro redes, todas ellas involucran un pequeño sistema de potencia, un conjunto de cargas, sistemas de generación y sistemas de almacenamiento de energía. La utilización de sistemas de generación distribuida renovable junto con la incorporación de sistemas de almacenamiento de energía representan un aporte beneficioso para asegurar tanto el funcionamiento sustentable como estable de las micro redes.

A partir de lo anterior, puede decirse que los conceptos de micro redes ofrecen características sobresalientes para los clientes del sistema de potencia en el sentido de que se puede mejorar la confiabilidad del sistema de potencia, la mayor calidad de la energía a través del control de la carga, la reducción de las emisiones de gases de efecto invernadero mediante la utilización de sistemas de generación de energía empleando recursos renovables, la operación económica debido a la reducción de costos de transporte y además es posible seleccionar las unidades de generación menos costosas.

Con el objetivo de analizar la estabilidad y el comportamiento dinámico de una micro red se han propuesto modelos linealizados de los dispositivos que la componen, como se muestra en Tang et al. (2009). Un análisis de autovalores y sus sensibilidades revela como este sistema está influenciado por la configuración y además por la variabilidad de la generación renovable. Si bien la linealización del sistema es útil en un entorno de trabajo, esta técnica no brinda suficientes garantías en un rango amplio de operación. A pesar de esto, importantes resultados pueden ponerse de manifiesto en cuanto a la variación del recurso renovable, la cual provoca una fluctuación en la potencia en la red eléctrica. Estas perturbaciones en el sistema de potencia pueden ser mitigadas mediante el empleo de sistemas de almacenamiento de energía (Tang et al., 2009, 2014).

En este sentido, los sistemas de almacenamiento de energía son de vital importancia para la operación de la micro red cuando funciona de forma aislada de la red de distribución. Además, la transición entre el estado conectado y desconectado de la micro red no se puede predecir debido a que la desconexión se produce ante una falla inesperada en la red de distribución. La transición entre el estado conectado y desconectado debe ser suave. En este contexto, la instalación de un sistema de almacenamiento de energía (ESS) con la capacidad de absorber y entregar energía con tiempos de respuesta lo suficientemente rápidos pueden ayudar a la estabilidad de la micro red (Espinar y Mayer, 2011).

El empleo del moldeo de potencia permite no solamente utilizar las variables tensiones y corrientes en el diseño del controlador sino que también permite considerar cargas de dinámicas no lineales en el sistema de potencia asegurando la convergencia a la estabilidad del mismo gracias al empleo de una función de Lyapunov.

\subsubsection{Topología base y componentes}

En esta tesis, como primera aproximación a las micro redes se estudió una topología en base a los trabajos (Toccaceli et al., 2012b,a, 2014), que se presenta en la Figura 6.13 y puede operar conectada a una red de distribución y también de forma aislada. El sistema está compuesto por: un aerogenerador, un generador sincrónico, un sistema de almacenamiento 
de energía y una carga conectada al bus.

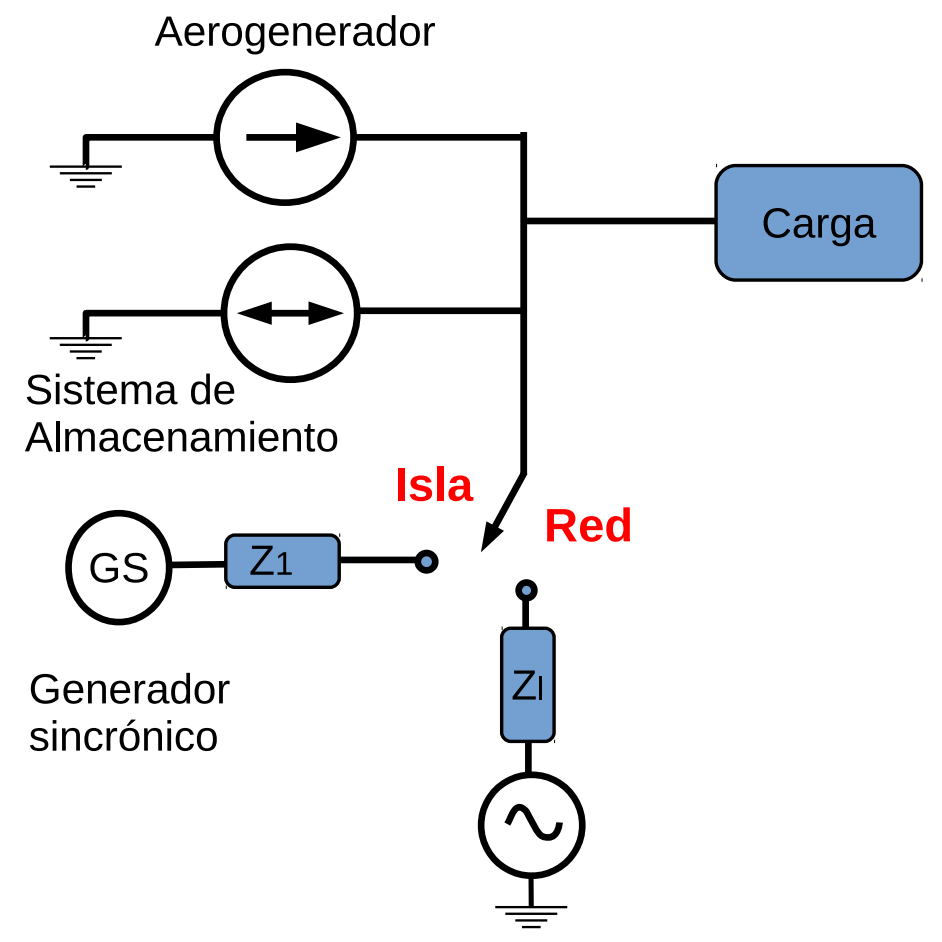

Figura 6.13: Esquema de la red considerada.

La operación de forma aislada se logra a partir del aporte de potencia del generador sincrónico y del aerogenerador, donde el primero impone la tensión y la frecuencia en la micro red. De esta forma, se puede asegurar la provisión de energía en los casos en que la red queda fuera de servicio.

En esta sección se considera la operación del sistema aislado, quedando como una etapa posterior a esta tesis el análisis de la conexión y desconexión de la red de distribución.

En la Figura 6.14 se muestra un esquema del sistema de potencia considerado durante la operación en isla. Este consiste de un generador sincrónico acoplado a un motor diésel $\left(G S_{1}\right)$, una línea de distribución $\left(Z_{1}\right)$, un aerogenerador $\left(G A_{1}\right)$, un sistema de almacenamiento de energía (ESS) y una carga dinámica considerando que son predominantemente motores de inducción conectados al bus 1. La línea considerada que une el generador sincrónico y el nodo 1 es un conductor de dos kilómetros de longitud con una resistividad de $0,065\left[\frac{\Omega}{\mathrm{km}}\right]$.

La adición de un ESS en un sistema de potencia puede tener múltiples objetivos. Con el objetivo de amortiguar variaciones de potencia, producto de variaciones rápidas en la carga, el ESS debe aportar energía con un tiempo de respuesta lo suficientemente rápido. En este trabajo de tesis el principal objetivo es controlar las potencias activa y reactiva que intercambia un sistema de almacenamiento de energía. En función de las necesidades del sistema de potencia el sistema de almacenamiento de energía puede estar compuesto por volantes de inercia o supercapacitores, los cuales tienen tiempos de respuestas del orden de los milisegundos, poseen buen ciclo de vida y además tienen un bajo costo por kWh (Espinar y Mayer, 2011; Boicea, 2014). Los ESS mencionados anteriormente se conectan a través de un convertidor de potencia. En el caso de las ruedas de inercia, el sistema de conexión se 


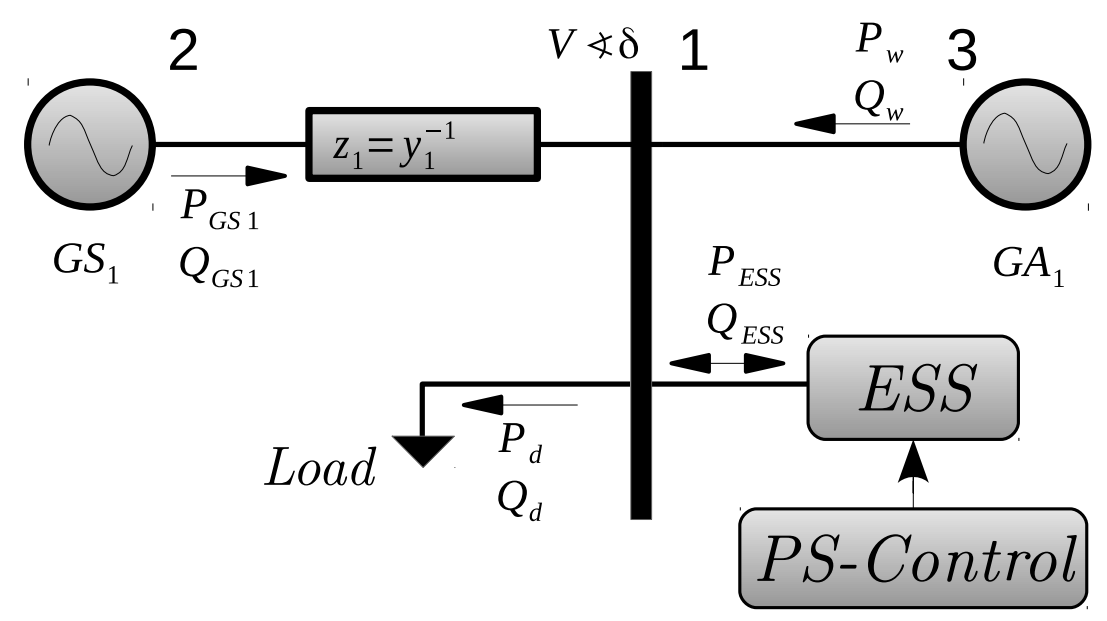

Figura 6.14: Esquema de la micro red considerada.

denomina indirecto empleando un convertidor espalda contra espalda con la misma estructura que se utiliza en el rotor de un aerogenerador con un DFIG. Mientras que un ESS basado en supercapacitores utiliza directamente un inversor electrónico para intercambiar energía con el punto de conexión de forma muy rápida. El cuello de botella en este ESS es el tiempo de respuesta del elemento de almacenamiento de energía, que en el caso de un supercondensador es del orden de los milisegundos.

Un convertidor asociado a un DFIG puede modificar la potencia que suministra en sus bornes de forma muy rápida en comparación con los tiempos de la red. De esta manera, se asume que el convertidor asociado al ESS tiene características similares a uno utilizado en un DFIG y por esta razón la dinámica asociada al convertidor del ESS no es considerada en comparación con los tiempos de la red eléctrica.

La dinámica del sistema considerado es capturada escribiendo las ecuaciones asociadas a la red eléctrica. La sumatoria de potencias activas para el sistema de la Figura 6.14 es:

$$
P_{n 1}-P_{d}+P_{w} \pm P_{E S S}=0 \Rightarrow P_{n 1}=P_{d}-P_{w} \pm P_{E S S}
$$

y la sumatoria de potencias reactivas en el sistema es:

$$
Q_{n 1}-Q_{d}+Q_{w} \pm Q_{E S S}=0 \Rightarrow Q_{n 1}=Q_{d}-Q_{w} \pm Q_{E S S}
$$

donde $P_{d}, Q_{d}, P_{w}, Q_{w}, P_{E S S}, Q_{E S S}, P_{n 1}$ y $Q_{n 1}$ son las potencias activas y reactivas consumida por la carga dinámica, inyectadas en el punto de conexión por el aerogenerador, las intercambiadas por el sistema de almacenamiento de energía y las que inyecta el generador sincrónico $\left(P_{G S 1}\right.$ y $\left.Q_{G S 1}\right)$ al nodo 1 a través de $Z_{1}$, respectivamente. Las potencias que intervienen en la red eléctrica se muestran en el esquema de la Figura 6.14.

Es posible escribir las ecuaciones de las potencias activa y reactiva para el sistema de la Figura 6.14 empleando las definiciones introducidas en el Capítulo 3. Entonces, para el sistema analizado se puede escribir la potencia activa para una línea resistiva en el nodo 1 como:

$$
P_{n 1}=\left(V_{1}\right)^{2} G_{11}+V_{1} V_{2} G_{11} \cos \left(\theta_{k m}\right)
$$


y la potencia reactiva como:

$$
Q_{n 1}=V_{1} V_{2} G_{11} \operatorname{sen}\left(\theta_{k m}\right)
$$

donde $V_{1}$ es la tensión en el nodo $1, V_{2}$ es la tensión en los bornes del generador sincrónico $G S_{1}, G_{11}$ es la conductancia de la línea que conecta el nodo 1 con el generador sincrónico y $\theta_{k m}$ es el ángulo de la tensión comprendido entre los nodos 1 y 2. Asumiendo, que el valor del ángulo en el nodo 2 es cero entonces $\theta_{k m}=\theta_{k}=\theta_{1}$.

\section{Ecuaciones del generador sincrónico}

Las expresiones que representan las características dinámicas de un generador sincrónico y del controlador de tensión del generador fueron presentadas en el Capítulo 3. Se asume que el par mecánico suministrado por el impulsor del generador sincrónico y el flujo son constantes. Con las consideraciones anteriores, las ecuaciones que describen la dinámica del generador sincrónico y el regulador de tensión son:

$$
\text { Generador Sincrónico }\left\{\begin{array}{clcc}
\dot{\delta_{1}} & = & \omega_{1}-\omega_{r}, \\
M_{1} \dot{\omega_{1}}+D_{1} \omega_{1} & = & P_{m}-P_{e}, \\
T_{A} \dot{E}_{f d i} & = & -E_{f d i}+K_{a}\left(V_{\text {ref }}-V\right),
\end{array}\right.
$$

donde las dos primeras expresiones son conocidas como las ecuaciones de oscilación (swing) y la última expresión representa el controlador de tensión del generador sincrónico. En la ecuación (6.77) $\omega_{1}$ es la velocidad angular, $\omega_{r}$ es la frecuencia de la línea de referencia, $M_{1}$ es la constante de inercia, $D_{1}$ es la fricción interna de la máquina, $P_{m}$ es la potencia suministrada por el impulsor, $P_{e}$ es la potencia eléctrica, $E_{f d i}$ es la tensión de campo, $V_{r e f}$ es la tensión de referencia para el regulador de tensión, $K_{a}$ y $T_{a}$ son constantes para el controlador de tensión.

\section{Carga dinámica}

Los modelos de cargas en un sistema eléctrico de potencia son de gran importancia para el análisis de estabilidad de tensión del sistema, la simulación y el control del sistema dinámico. En particular, para analizar los problemas asociados a los colapsos de tensión, un modelo estático para la carga no es adecuado para caracterizarla.

En los sistemas de potencia, y en particular en aquellos que incorporan sistemas de generación distribuida renovable es de importancia analizar la incidencia de las máquinas de inducción como cargas. Generalmente, estos sistemas de potencia tienen un vínculo débil con los sistemas eléctricos externos. Es de notar que, un porcentaje del 60 al $70 \%$ de las cargas son motores de inducción conectados a la red eléctrica (Kundur, 1993).

La carga dinámica considerada en el esquema de la Figura 6.14, desarrollada en el Capítulo 3, es:

$$
\begin{gathered}
P_{d}=P_{0}+P_{1}+k_{p w} \dot{\delta}+k_{p v}(V+T \dot{V}), \\
Q_{d}=Q_{0}+Q_{1}+k_{q w} \dot{\delta}+k_{q v} V+k_{q v 2} V^{2},
\end{gathered}
$$

donde $P_{0}$ y $Q_{0}$ son las potencias activa y reactiva constantes, $P_{1}$ y $Q_{1}$ representan la carga $\mathrm{PQ}, V$ es la tensión en el punto de conexión y $\dot{\delta}$ es la frecuencia. Los parámetros en p.u. 
para las ecuaciones (6.78) y (6.79) se presentan en Dobson y Chiang (1989) y son: $k_{p w}=0,4$, $k_{p v}=0,3, T=8,5, k_{q w}=-0,03, k_{q v}=-2,8, k_{q v 2}=2,1, P_{0}=0,6$ у $Q_{0}=1,3$.

Luego, a partir de la expresiones de las potencias activa y reactiva (6.78) y (6.79) de la carga dinámica y teniendo en cuenta los diferentes aportes de potencia es posible obtener las siguientes expresiones para $\dot{\delta}$ y $\dot{V}$ :

$$
\dot{\delta}=\frac{\left(Q_{A}-Q_{0}-Q_{1}-k_{q v} V-k_{q v 2} V^{2}\right)}{k_{q w}},
$$

$\operatorname{con} Q_{A}=Q_{n 1}+Q_{w} \pm Q_{E S S}$

$$
\dot{V}=\frac{\left(P_{A}-P_{0}-P_{1}-k_{p w} \dot{\delta}-k_{p v} V\right)}{\left(k_{p v} T\right)}
$$

$\operatorname{con} P_{A}=P_{n 1}+P_{w} \pm P_{E S S}$.

\section{Modelo de estados}

Si el aerogenerador conectado al nodo 1 de la red de la Figura 6.14 está aportando una potencia constante al nodo, el modelo de estados dominantes a partir de las consideraciones previas está compuesto por la carga dinámica y el generador sincrónico, explicados anteriormente.

$$
\left\{\begin{array}{l}
\dot{x}=f(x)+g(x) u \\
y=h(x)
\end{array},\right.
$$

donde $x^{T}=\left[\begin{array}{llll}\omega_{1} & E_{f d i} & \delta & V\end{array}\right], u$ es el vector de entrada. Las ecuaciones que constituyen $f(x)$ y $g(x)$ corresponden a la carga dinámica y al generador sincrónico.

De esta manera el sistema queda definido como:

$$
f(x)=\left[\begin{array}{l}
f_{1}(x) \\
f_{2}(x) \\
f_{3}(x) \\
f_{4}(x)
\end{array}\right],
$$

donde

$$
\begin{aligned}
f_{1}(x) & =\left(\frac{P_{m}-P_{e}-D_{1} x_{1}}{M_{1}}\right) \\
f_{2}(x) & =\frac{\left(-x_{2}+K_{a}\left(V_{r e f}-V\right)\right)}{T_{a}} \\
f_{3}(x) & =\frac{\left(Q_{A}-Q_{0}-Q_{1}-k_{q v} x_{4}-k_{q v 2} x_{4}^{2}\right)}{k_{q w}}, \\
f_{4}(x) & =\frac{\left(P_{A}-P_{0}-P_{1}-k_{p w} \dot{x_{3}}-k_{p v} x_{4}\right)}{\left(k_{p v} T\right)} .
\end{aligned}
$$

y por el vector:

$$
g(x)=\left[\begin{array}{cc}
0 & 0 \\
0 & 0 \\
\frac{1}{k_{q w}} & 0 \\
0 & \frac{1}{\left(k_{p v} T\right)}
\end{array}\right]
$$




\subsubsection{Ley de control a partir de moldeo de potencia}

Para aplicar la técnica de control por moldeo de potencia para el sistema de almacenamiento de energía es necesario representar el sistema dinámico (6.82) utilizando las ecuaciones de Brayton-Moser (6.13). En este sentido, es posible proponer pares alternativos $(\tilde{Q}(x)$ y $\tilde{P}(x))$ que pueden asegurar la condición $\tilde{Q}(x)+\tilde{Q}(x)^{T} \leq 0^{1}$

El nuevo par $\tilde{Q}(x)$ y $\tilde{P}(x)$ representan el sistema dado por

$$
\tilde{Q} \dot{x}=\nabla \tilde{P}+\tilde{G} u,
$$

siendo

$$
\tilde{Q}(x)=\left[\frac{1}{2} \nabla(Q f) M+\frac{1}{2} \nabla^{T}(M Q f)+\lambda I\right] Q,
$$

donde $\lambda \in \mathbb{R}$ y $M=M^{T}: \mathbb{R}^{n} \rightarrow \mathbb{R}^{n x n}$, son arbitrarios.

A su vez, $\tilde{P}(x)$ está dado por:

$$
\tilde{P}(x)=\lambda \int(Q f)^{T} d x+\frac{1}{2} f^{T} Q^{T} M Q f
$$

$\mathrm{y}$

$$
\tilde{G}=\tilde{Q} g .
$$

Las matrices $M$ y $Q$ propuestas determinan una estructura simétrica para la matriz $\tilde{Q}$. La elección de las matrices $M, Q$ y el aniquilador a izquierda de $g(x)$ brinda una forma para la PDE.

El aniquilador a izquierda de $g(x)$ propuesto, por una cuestión de simplicidad, para este problema es:

$$
g^{\perp}(x)=\left[\begin{array}{llll}
1 & 0 & 0 & 0 \\
0 & 1 & 0 & 0
\end{array}\right] .
$$

Para el diseño de un par alternativo $(Q(x)$ y $P(x))$ se seleccionó:

$$
\begin{gathered}
M=0_{4}, \\
\tilde{Q}(x)=-1 I_{4} .
\end{gathered}
$$

La ecuación en derivadas parciales empleando la matriz alternativa $\tilde{Q}(x)$ y el aniquilador a izquierda de $g(x)$ es:

$$
g^{\perp} \tilde{Q}^{-1} \nabla P_{a}(x)=\left[\begin{array}{l}
q_{11} \frac{\partial P_{a}(x)}{\partial x_{1}} \\
q_{22} \frac{\partial P_{a}(x)}{\partial x_{2}}
\end{array}\right]=0 .
$$

En esta última expresión nosotros podemos observar que la función $P_{a}(x)$ no depende de las variables $x_{1}$ y $x_{2}$. De esta forma se puede proponer una función $P_{a}(x)$, que sólo dependa de las variables $x_{3}$ y $x_{4}$, respectivamente. Además, la función candidata de Lyapunov

\footnotetext{
${ }^{1}$ Como parte de los trabajos realizados, el empleo de pares alternativos también fue utilizado en Fernández et al. (2015) para proponer una ley de control para un generador sincrónico de imanes permanentes.
} 
$P_{d}(x)=P(x)+P_{a}(x)$ debe alcanzar un mínimo local en el punto de equilibrio $x^{*}$, es decir $\left(\nabla P_{d}\left(x^{*}\right)=0\right.$ y $\left.\nabla^{2} P_{d}(x)>0\right)$. La función $P_{a}(x)$ fue diseñada considerando que el sistema de almacenamiento de energía tiene una cantidad de energía finita. El ESS solamente puede entregar energía cuando el sistema se ve afectado por una perturbación. La función $P_{a}(x)$ propuesta está dada por:

$$
P_{a}(x)=k_{1}\left(x_{3}-x_{3}^{*}\right)^{2}+k_{2}\left(x_{4}-x_{4}^{*}\right)^{2} .
$$

\section{Verificación del Hessiano}

Para verificar que $P_{d}(x)$ es una función candidata de Lyapunov, el Hessiano debe ser definido positivo en el punto de equilibrio. El Hessiano está dado por:

$$
\nabla^{2} P_{d}(x)=\left(\begin{array}{cccc}
\frac{D_{i}}{M_{i}} & 0 & 0 & 0 \\
0 & \frac{1}{T_{A}} & 0 & 0 \\
0 & 0 & 2 k_{1} & 0 \\
0 & 0 & 0 & 2 k_{2}
\end{array}\right)>0
$$

La condición $\left(\nabla^{2} P_{d}\left(x^{*}\right)>0\right)$ puede ser verificada chequeando los menores principales de la matriz (6.98) para lo cual pueden ajustarse los valores de $k_{1}$ y $k_{2}$.

La ley de control empleando la expresión (6.48) está dada por:

$$
u=\left[\begin{array}{c}
-k_{q w} \frac{\partial P_{a}(x)}{\partial x_{3}} \\
-k_{p v} T \frac{\partial P_{a}(x)}{\partial x_{4}}
\end{array}\right] .
$$

El ajuste del controlador tiene interpretación a partir de la expresión (6.97) que moldea la función de potencial mixto $P(x)$. En efecto, a partir del ajuste de $k_{1}$ y $k_{2}$ en la expresión de $P_{a}(x)$ se puede lograr modificar la función de potencia total $P_{d}(x)$ realimentando en una dirección especifica.

\section{Acondicionamiento de la referencia mediante modo deslizante}

Con el objetivo de considerar las limitaciones físicas de un ESS con respecto a las potencias activa y reactiva que puede intercambiar con el sistema de potencia es posible aplicar un método de acondicionamiento de la referencia. En particular, se utilizaron conceptos de acondicionamiento de la referencia por modo deslizante (SMRC, sliding mode reference conditioning) para modificar la referencia para la ley de control para el ESS (Garelli et al., 2011).

El lazo de acondicionamiento del ESS es mostrado en la Figura 6.15. Por una cuestión de claridad, el lazo correspondiente a la potencia reactiva se ha omitido. Cuando el ESS trabaja en el rango de potencias permitidas, es decir $P^{-}=P_{\text {lim }}^{-} \leq P \leq P_{\text {lim }}^{+}=P^{+}$la señal $w$ es cero y el lazo de acondicionamiento de la referencia no está activo. El lazo se activa cuando se supera su cota superior, $s=P^{+}{ }_{-} P<0$, $w$ cambia a $w^{-}$. De forma similar, si $P$ cae por debajo de su límite inferior, la variable $w$ conmuta a $w^{+}$. 


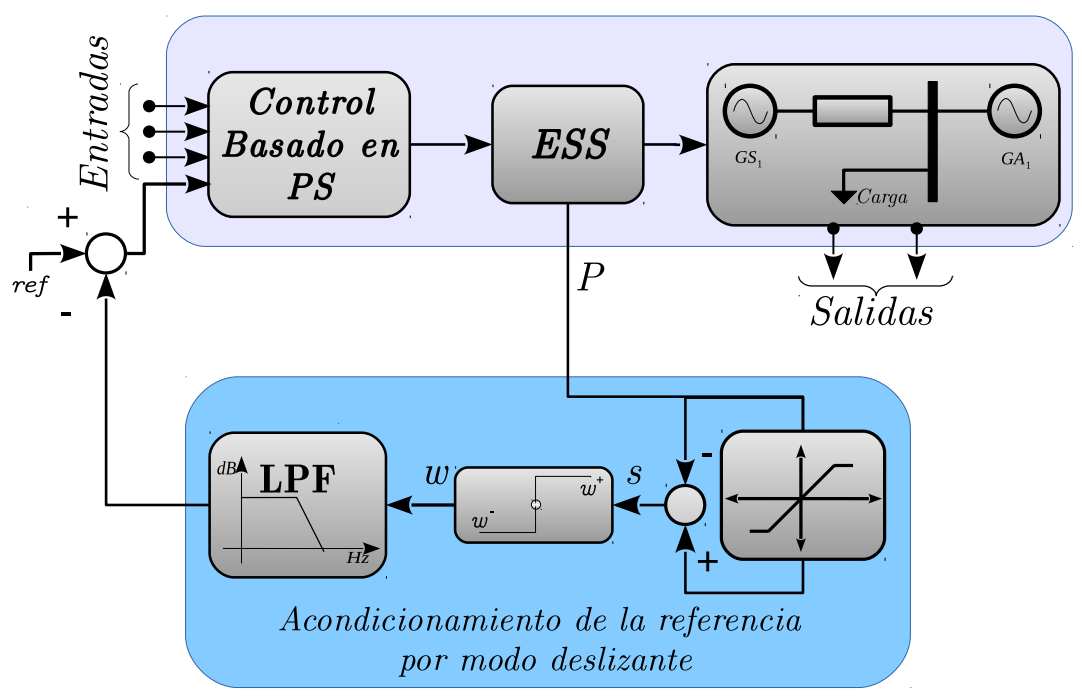

Figura 6.15: Esquema de la implementación del lazo de acondicionamiento de la referencia.

A partir de un punto de vista práctico, debido a que la dinámica de la red eléctrica es lenta, se asume que las conmutaciones de $w$ directamente afectan a la primera derivada del tiempo de la señal limitada $P$ y de la función de conmutación $s$. Además, asumiendo una señal discontinua los suficientemente grande $w$ la lógica de conmutación asegura que:

$$
\begin{cases}\dot{s}>0 & \text { si } s<0 \\ \dot{s}<0 & \text { si } s>0\end{cases}
$$

De esta manera, cuando $P$ supera uno de sus límites, $w$ modifica la referencia para asegurar que la variable $P$ regrese a su región de funcionamiento permitida, donde la variable $w$ es igual a cero. Mientras la variable $P$ intente superar el límite de potencia del ESS, la señal $w$ conmutará entre $0 \mathrm{y} w^{-}$en alta frecuencia y el sistema evolucionará de manera que $s=0$. En consecuencia, la señal de referencia acondicionada se ajustará continuamente de forma tal que la salida $P$ nunca exceda el límite.

\subsubsection{Evaluación de la ley de control}

En la Figura 6.16 se pueden observar las curvas de nivel sobre el plano $\left(x_{3}, x_{4}\right)$ y la superficie que representa a la función de potencial mixto $P(x)$, es decir de lazo abierto. Como se observa en la Figura (6.16), el sistema eléctrico de potencia tiene un punto de equilibrio, el cual puede ser calculado a partir de las ecuaciones que describen el sistema, y es representado como $x^{*}$.

En la Figura (6.16) también puede observarse que si bien existe un punto de equilibrio las curvas de nivel se abren en la región cercana a dicho punto.

La función $P_{a}(x)$ propuesta para este problema, la cual repetimos a continuación para mejor claridad, modifica la función a lazo abierto en:

$$
P_{a}(x)=k_{1}\left(x_{3}-x_{3}^{*}\right)^{2}+k_{2}\left(x_{4}-x_{4}^{*}\right)^{2} .
$$




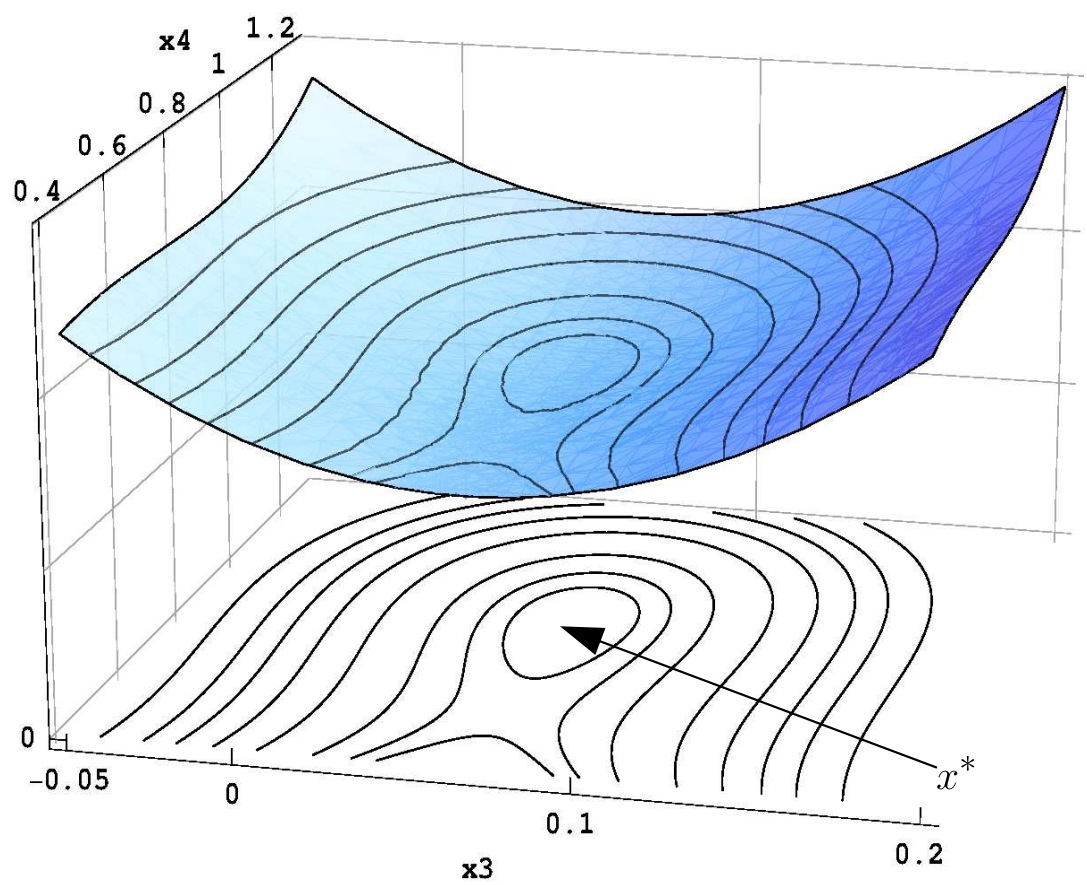

Figura 6.16: Curvas de nivel y superficie de la función $P(x)$ (lazo abierto).

Observando la Figura 6.16, el dominio de atracción puede ser ampliado modificando la función $P(x)$ en la dirección de la variable $x_{4}$. Esto es posible modificando el valor de $k_{2}$ en la función $P_{a}(x)$, la cual modifica la función $P_{d}(x)$ en dicha dirección.

En la Figura 6.17 se muestra la función de lazo cerrado $P_{d}(x)$ y las curvas de nivel sobre el plano para una elección de las constantes $k_{1}$ y $k_{2}$. En este sentido, también modificando estas constantes es posible modificar la función $P_{a}(x)$ para que el sistema realimentado alcance el punto de equilibrio rápidamente. Además, en la Figura 6.18 se representan sobre el plano $x_{3}-x_{4}$ las curvas de nivel de las funciones de potencial mixto $P(x)$ y la función de lazo cerrado $P_{d}(x)$. Con líneas de trazos se representa la función $P(x)$ y con línea sólida se dibuja la función $P_{d}(x)$, el punto de equilibrio es $x^{*}$.

En la Figura 6.19 se muestran las funciones $P_{d}(x)$ para dos elecciones de $k_{1}$ y $k_{2}$. En la Figura 6.19 a) se configura la función $P_{a}(x)$ con $k_{1}=k_{2}=1$ y en la Figura $6.19 \mathrm{~b}$ ) los valores configurados con $k_{1}=1$ y $k_{2}=2$. Estas dos elecciones de ganancias permiten observar en las curvas de nivel de la Figura 6.19 cómo la función $P_{a}(x)$ consigue dar forma a la función de potencial mixto. Además, con esta elección de $P_{a}(x)$ se logra modificar la curvatura de la función $P_{d}(x)$ sobre uno de los ejes de forma simple. De esta manera, se puede lograr que las curvas de nivel de la función de lazo cerrado cubran una mayor vecindad del punto de equilibrio, como se puede observar en la Figura 6.18. 


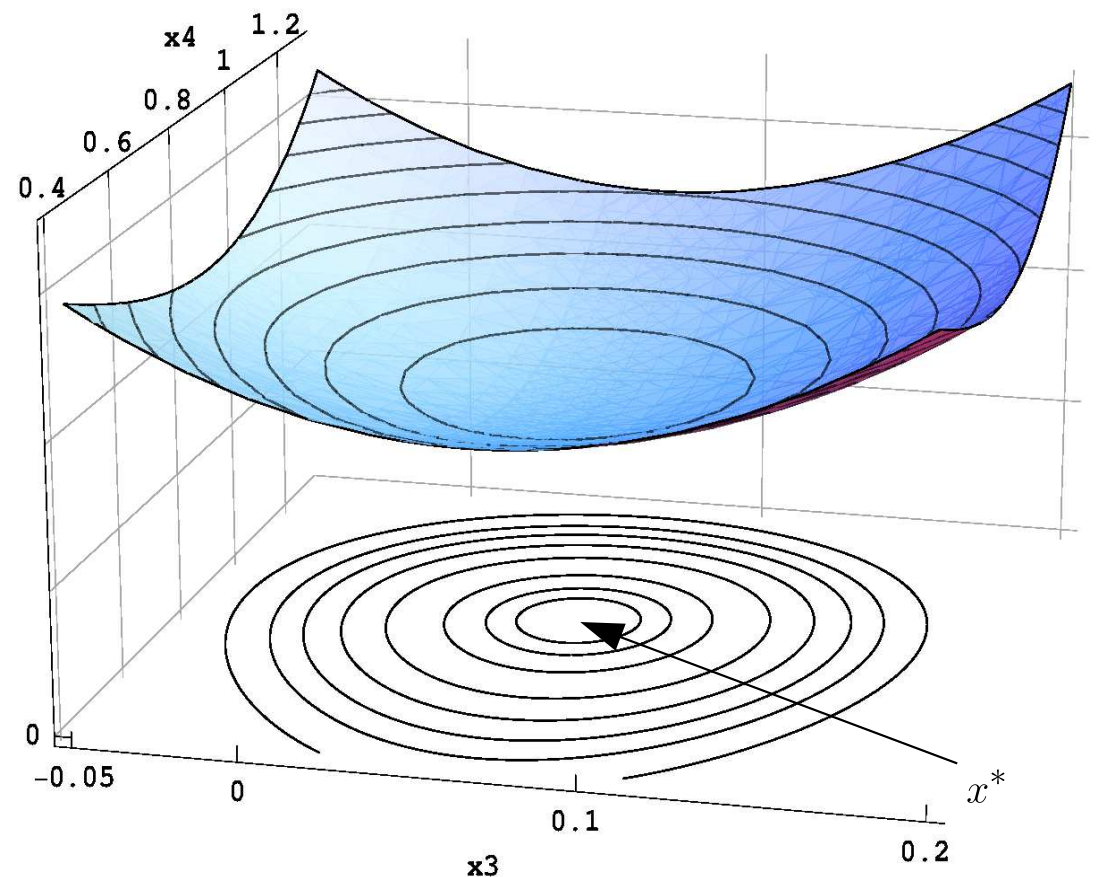

Figura 6.17: Curvas de nivel y superficie de la función $P_{d}(x)$.

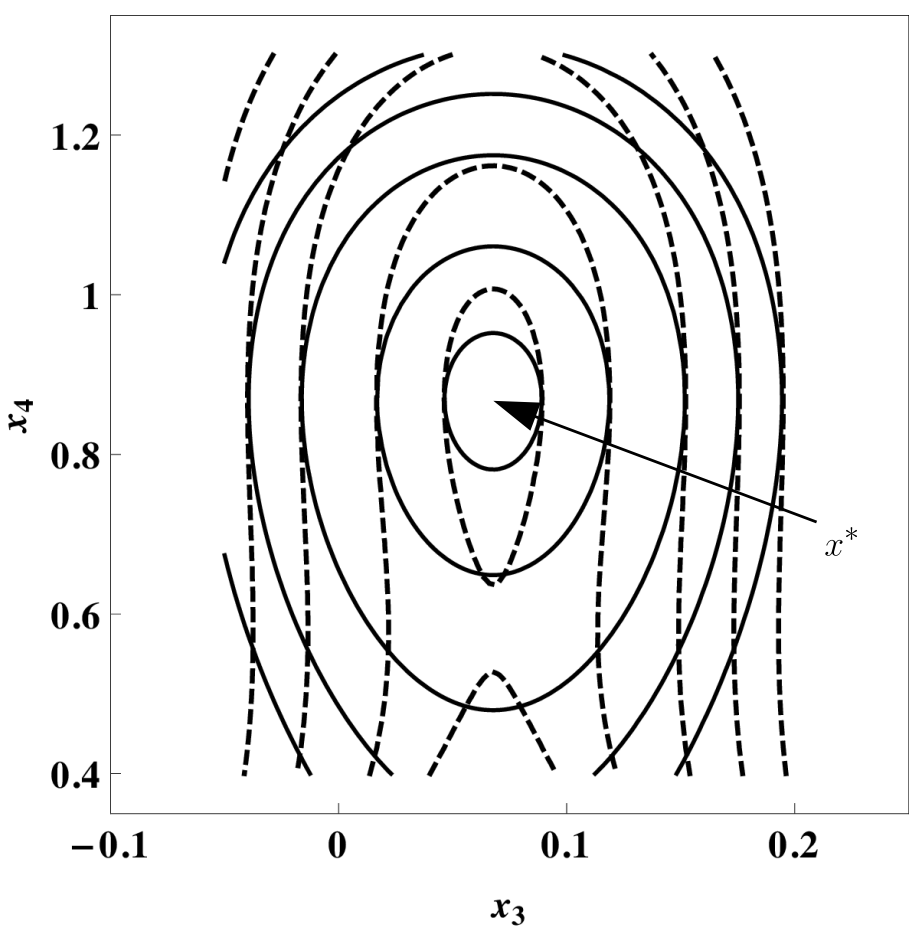

Figura 6.18: Curvas de nivel de las funciones $P(x)$ (líneas de trazos) y $P_{d}(x)$ (en líneas llenas).

\section{Resultados de simulación}

Para evaluar el desempeño de los controladores, se consideran perturbaciones en las potencias activa y reactiva sobre el nodo 1 de la Figura 6.14. Las mismas fueron diseñadas tanto para evaluar el buen comportamiento del control por moldeo de potencia como el 

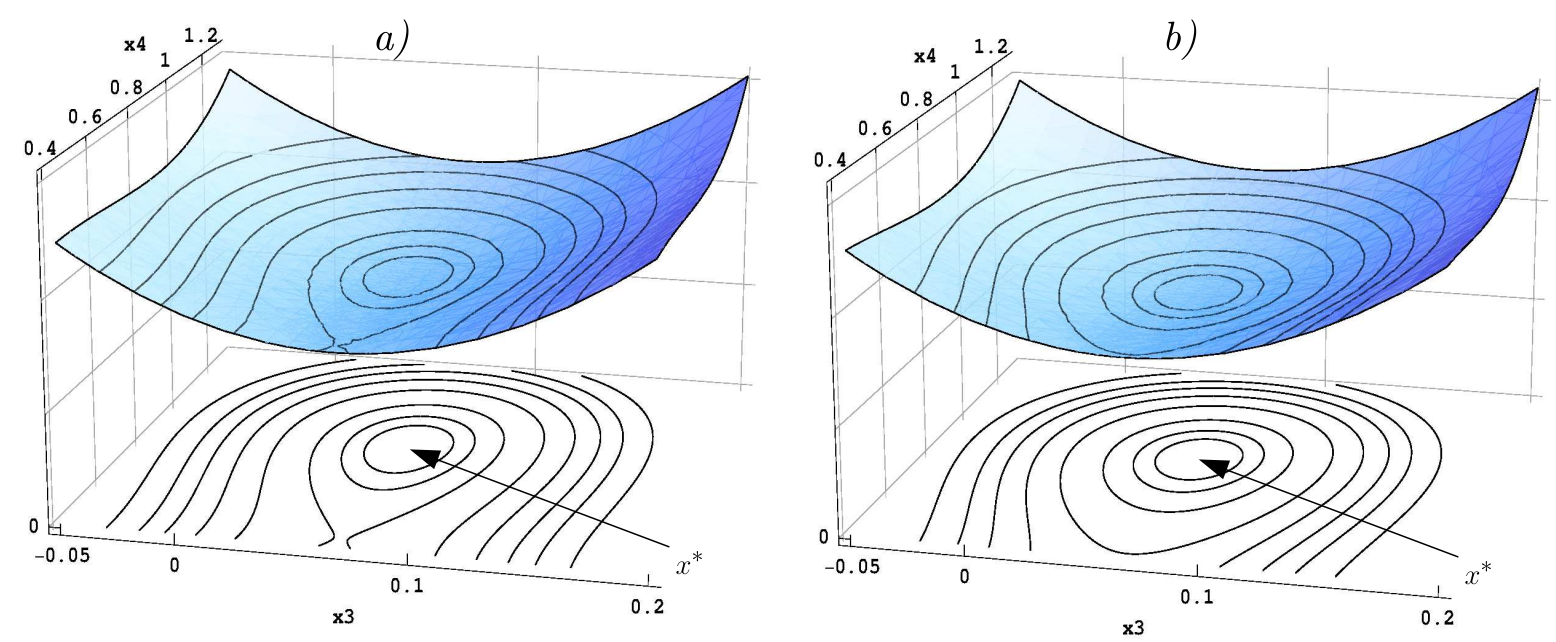

Figura 6.19: Curvas de nivel y superficie de la función $P_{d}(x)$ para dos elecciones del par $\left(k_{1}, k_{2}\right)$.

correcto funcionamiento del sistema de acondicionamiento de señal (Figura 6.15). Los límites máximos para el aporte de potencia activa y reactiva para el ESS son: $P_{\max }=200 \mathrm{~kW}$ y $Q_{\max }=75 \mathrm{kVAr}$.

Dos casos han sido analizados, en el primer caso se considera una perturbación de potencia activa mientras que en el segundo caso se considera una de potencia reactiva. Las variables de estado del sistema eléctrico, tensión y ángulo se presentan en la Figura 6.20, en línea sólida se muestran cuando actúa el control y en línea de trazos cuando el mismo no está conectado. En dicha figura se observan las ventajas del control aplicado al ESS sobre las variables eléctricas. En particular, la tensión para los dos tipos de perturbaciones de potencia mejora con la adición de un ESS. Un comportamiento similar puede observarse en el ángulo. Por otra parte, en las Figuras 6.21 y 6.22 se muestran las potencias activa y reactiva que intercambian los elementos del sistema eléctrico considerado. En las mismas se representa con línea continua cuando interviene el ESS, mientras que con líneas de trazos cuando el ESS no esta conectado.

En la Figura 6.21 se presenta el primero de los casos. Una perturbación de $250 \mathrm{~kW}$ se aplica a $\mathrm{t}=1,5$ segundos, Figura 6.21 a), y se incrementa a $375 \mathrm{~kW}$ en $\mathrm{t}=2,5$ segundos desapareciendo a los 5 segundos. Es de notar, que perturbaciones importantes en la potencia activa pueden ser ocasionadas tanto por causa de variaciones en el recurso eólico como por variaciones en la carga.

Mientras que la contribución de las potencias activa del generador sincrónico y del aerogenerador $\left(P_{w}+P_{G S 1}\right)$ junto con la potencia de la carga es mostrada en la Figura 6.21 b) y c), respectivamente, en la Figura 6.21 d) es presentada la acción de control del ESS, es decir la potencia intercambiada por el sistema de almacenamiento de energía.

El control en $t>1,5$ segundos detecta la variación de tensión que se muestra en la Figura 6.20 a) y el ESS contribuye con potencia activa según indica la Figura 6.21 d). En dicha figura el sistema de almacenamiento de energía alcanza su máximo en $t>2,5$ segundos verificando el correcto funcionamiento de la técnica de acondicionamiento de señal adecuando el valor de la entrada de referencia del ESS para que su salida no supere el valor máximo.

En la Figura 6.21 b) se puede observar que sin la contribución del ESS (línea de trazos), el 

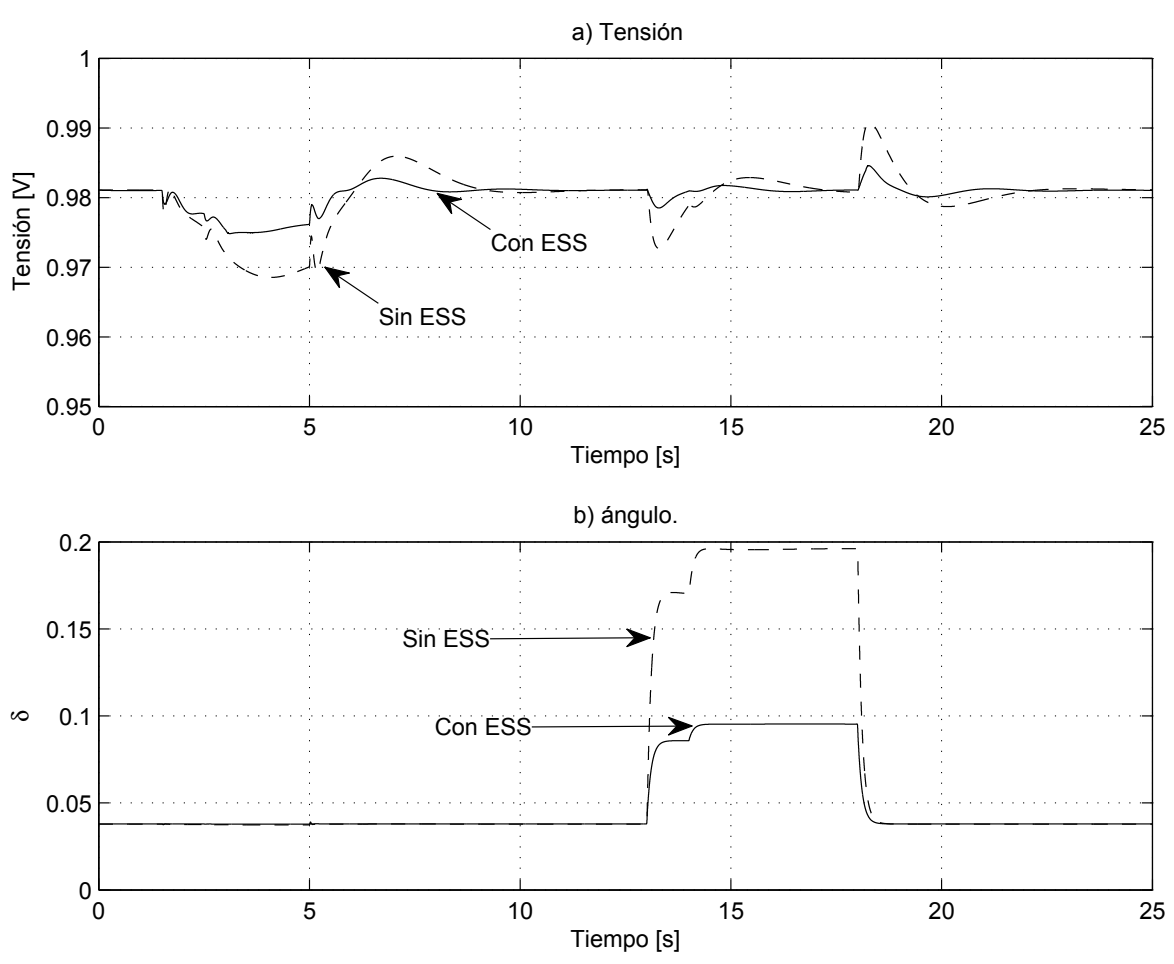

Figura 6.20: Tensión y $\delta$ en el nodo 1.

generador sincrónico tiene que suministrar toda la potencia requerida por la perturbación si el aerogenerador entrega potencia constante. La contribución del sistema de almacenamiento de energía (línea continua) reduce la potencia suministrada por el generador sincrónico, de esta manera considerando mucho ciclos de carga y descarga del ESS se minimiza tanto el consumo de combustible del motor diésel como el esfuerzo en el eje.

El segundo caso considerado es una perturbación en la potencia reactiva, el cual se presenta en la Figura 6.22.

Una perturbación de $100 \mathrm{kVAr}$ se aplica en $t=13$ segundos, Figura 6.22 a), y se incrementa a $120 \mathrm{kVAr}$ en $t=14$ segundos desapareciendo a los 18 segundos. Es de notar, que perturbaciones importantes en la potencia reactiva pueden ser ocasionadas por arranques de motores de inducción conectados en la micro red. La contribución de las potencias reactiva del generador sincrónico $\left(Q_{G S 1}\right)$, considerando que el aerogenerador esta configurado como reactivo neutro y la potencia de la carga son mostradas en la Figura 6.22 b) y c), respectivamente. En la Figura 6.22 d) se presenta la acción de control del ESS, en $t>13$ segundos detecta la variación del ángulo que se muestra en la Figura 6.20 b) y el ESS contribuye con potencia reactiva.

En dicha figura el sistema de almacenamiento de energía alcanza su máximo en $t>14$ segundos verificando el correcto funcionamiento de la técnica de acondicionamiento de señal adecuando el valor de la entrada de referencia del ESS para que su salida no supere el valor máximo. En la Figura 6.22 se observa que una leve perturbación en la potencia reactiva causa una variación en la tensión del nodo 1 y a su vez un cambio mayor en $\delta$, como se observa en Figura 6.20. 


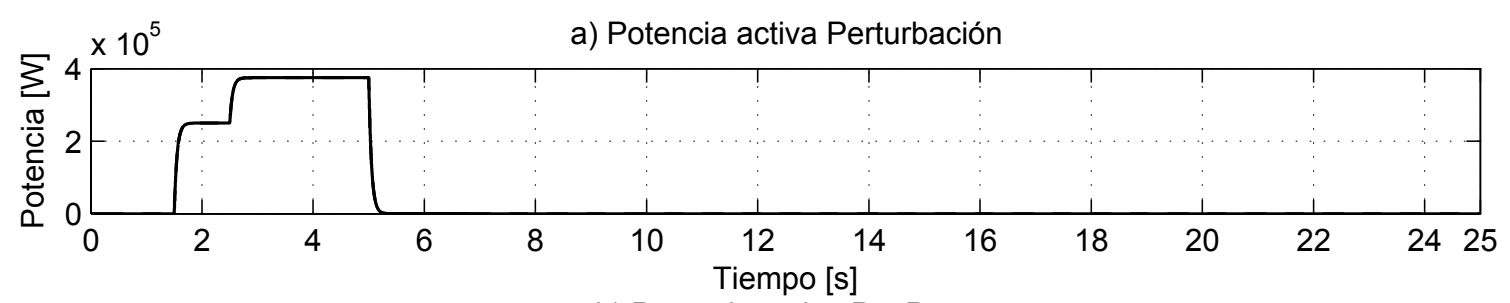

b) Potencia activa $\mathrm{P}_{\mathrm{w}}+\mathrm{P}_{\mathrm{GS} 1}$
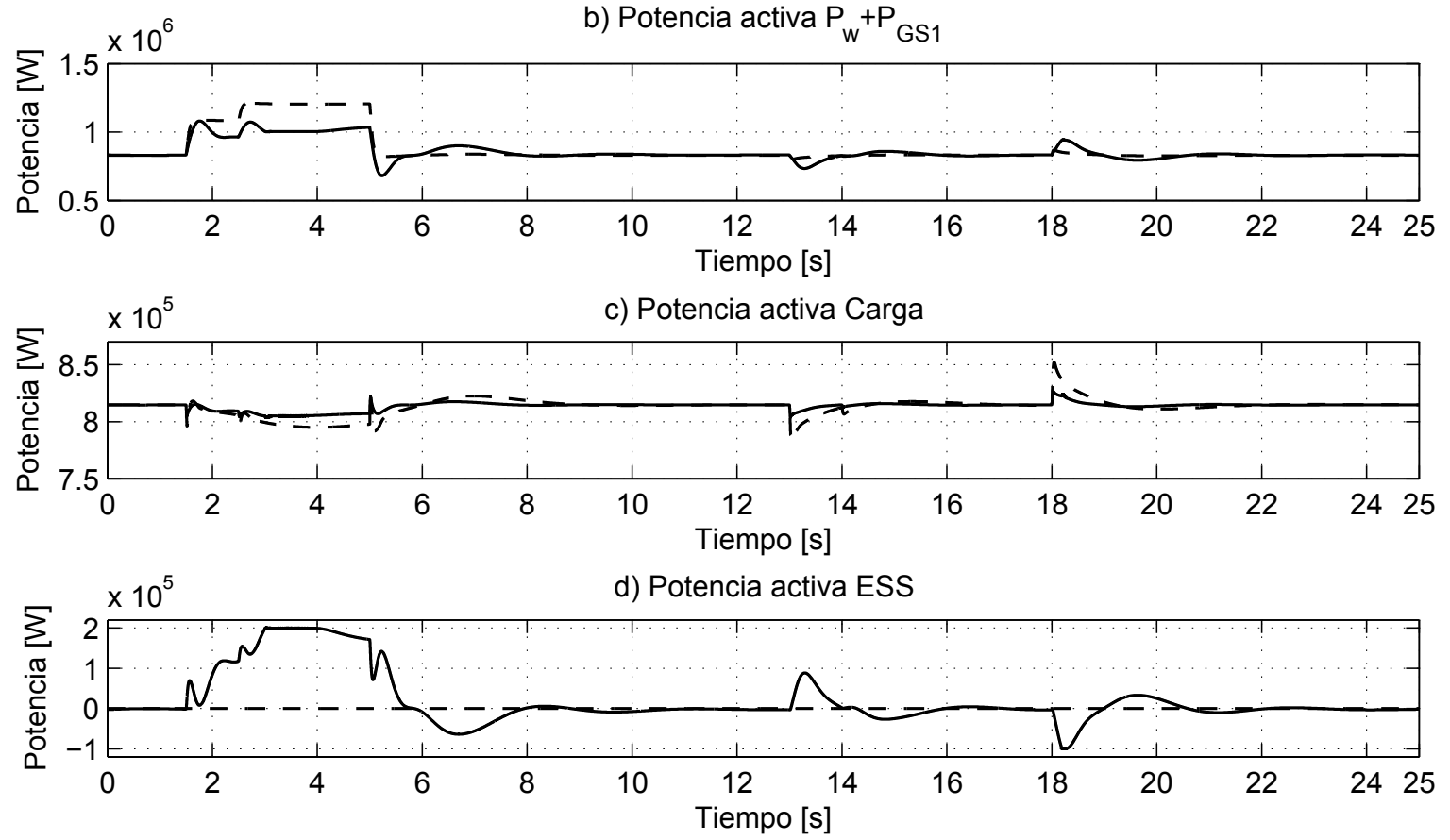

Figura 6.21: Potencia activa en la red eléctrica (en línea continua se representa cuando interviene el ESS y en línea de trazos cuando el ESS no participa). 


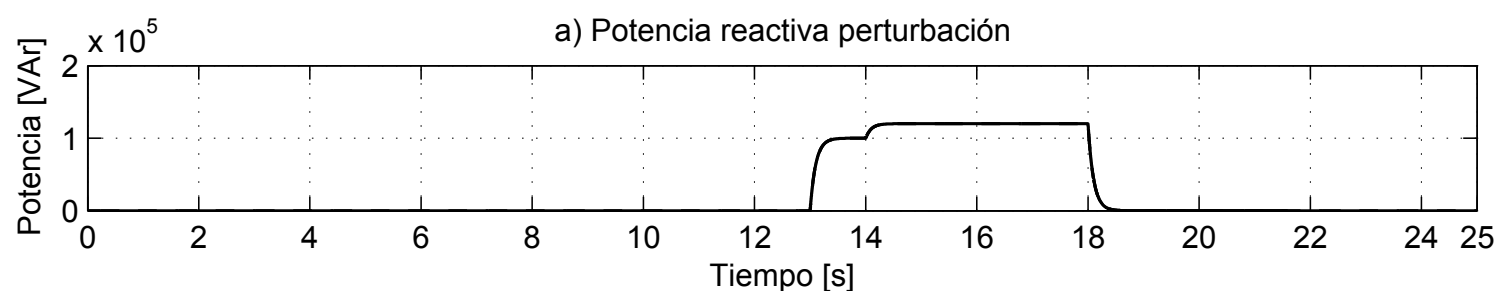

b) Potencia reactiva $\mathrm{GS}_{1}$

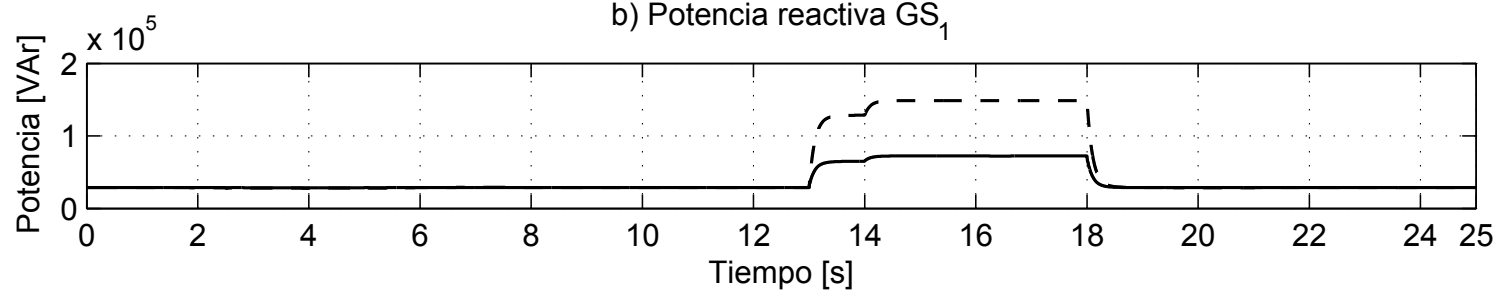

c) Potencia reactiva carga
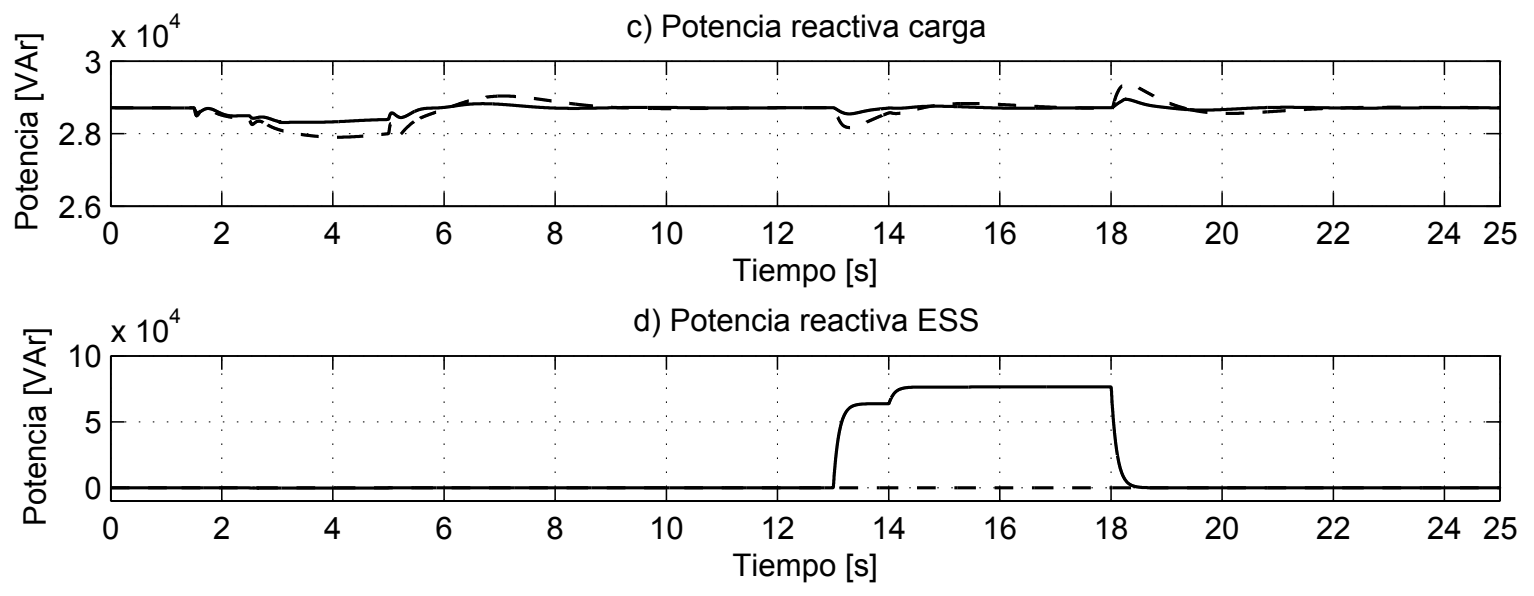

Figura 6.22: Potencia reactiva en la red eléctrica (en línea continua se representa cuando interviene el ESS y en línea de trazos cuando el ESS no participa). 


\subsection{Retrospección}

En este capítulo se analizó el control de la potencia activa y reactiva para un aerogenerador equipado con un generador de rotor bobinado y el control de potencia activa y reactiva de un sistema de almacenamiento de energía.

La técnica de control empleada para realizar el control de la máquina y para el sistema de almacenamiento de energía es balance de potencia, la cual garantiza la estabilidad del sistema. Además, sobre las estrategias de control basadas en pasividad se agregó una acción de control integral la cual robustece la implementación de los controladores frente a perturbaciones constantes. La estrategia de control propuesta permite extender los resultados a un parque eólico utilizando los conceptos del aerogenerador equivalente.

En el marco de una micro red, el control de un sistema de almacenamiento de energía conectado a una pequeña micro red es añadido con el objetivo de mejorar la calidad de la energía eléctrica en el punto de conexión y además para mejorar el aprovechamiento del recurso eólico renovable. Con respecto a la técnica de control cabe destacar que la acción de control propuesta no requiere la coordinación con los otros controladores de la micro red analizada y que además mediante el ajuste de las ganancias de la función $P_{a}(x)$ es posible modificar la función de potencia total $\left(P_{d}(x)\right)$ en una dirección especifica. En este sentido, el diseño presentado permite aumentar el dominio de atracción del sistema realimentado.

También se implementó un sistema de acondicionamiento de la referencia mediante modo deslizante con el objetivo de adecuar los valores de entrada de referencia del ESS para que su salida no supere los límites de potencia. 


\section{Capítulo 7}

\section{Conclusiones}

\subsection{Evaluación de resultados}

Los trabajos que han dado lugar a esta tesis se centraron en dos aspectos. El primero de ellos consistió en el control de un aerogenerador DFIG y el segundo en el de un sistema de almacenamiento para una micro red. En ambos casos la técnica utilizada fue Control Basado en Pasividad, en particular Moldeo de Potencia.

En principio se realizó el control de las potencias activa y reactiva de un aerogenerador DFIG. El agregado de una acción integral se realizó en el marco de la Teoría de Pasividad asegurando la estructura de Brayton-Moser del conjunto lo que garantiza las propiedades de robustez del control propuesto. Por otra parte, el enfoque anterior se extendió al control de una granja eólica compuesta por 6 generadores eólicos explotando el concepto de aerogenerador equivalente. Al igual que para el control de un aerogenerador se diseñó un controlador desacoplado para las potencias activa y reactiva.

Los controladores desarrollados en este trabajo de tesis fueron evaluados para condiciones severas tales como variaciones abruptas en el viento y en la tensión en el sistema de potencia al cual se encontraban conectados los DFIG mediante una línea de transmisión.

Dos casos fueron considerados para el control de la potencia reactiva que aporta la granja eólica a la red eléctrica. Con el objetivo que la máquina de inducción equivalente no consuma potencia reactiva de la red se diseñó una estrategia que cumpla con este objetivo denominada neutral a la red. La segunda estrategia propuesta, denominada regulación de tensión, tuvo como objetivo aportar potencia reactiva al punto de conexión en función de la desviación de tensión. En este caso, a pesar de que los aerogeneradores no tienen la capacidad de solucionar la falla que se produce en la red eléctrica, contribuyen aportando potencia reactiva.

La estrategia por regulación de tensión presentó un mejor comportamiento que la estrategia denominada neutral a la red frente a una variación de la tensión en el bus infinito. 
Tanto para el control de la potencia activa como reactiva, la adición del término integral aseguró la convergencia al valor de referencia de estado estacionario. A partir del análisis de una caída de tensión desbalanceada en el bus infinito, se puede observar un comportamiento característico que será evaluado en tareas de investigación futuras.

Por otra parte, el segundo aspecto estudiado involucró una micro red basada en un generador sincrónico, un aerogenerador, un sistema de almacenamiento y una carga dinámica que representa el consumo. Debido a que un porcentaje grande de la carga en una red eléctrica son motores de inducción, la carga dinámica fue representada por éstos. Luego, el modelo de carga equivalente presentó características no lineales.

Las acciones de control que contribuyeron a estabilizar y a amortiguar las variaciones de potencia en una micro red fueron implementadas sobre un ESS. Para la síntesis del controlador MIMO que se implementó sobre las potencias activa y reactiva del ESS, se utilizó Moldeo de Potencia.

Adicionalmente, se pudo verificar el incremento en el dominio de atracción garantizado por la nueva función de Lyapunov cuando ésta se comparó frente a la función de Energía obtenida con el control del generador sincrónico únicamente. También se mostró que, mediante el ajuste de la función potencia $P_{a}(x)$, es posible modificar la función de potencia total $\left(P_{d}(x)\right)$ en una dirección especifica. Lo anterior, permitiría ajustar la convergencia a la estabilidad del sistema a lazo cerrado en micro redes que posean inconvenientes en su estabilidad en la dirección de alguna de las variables de estado a lazo abierto.

Por otro lado, la acción de control propuesta no requiere coordinación con otros controladores a través de la estructura analizada. Esto permitiría estudiar casos para utilizar una estrategia de control del aerogenerador tendiente a estabilizar la micro red cuando el mismo posea capacidad de actuación.

\section{2. $\quad$ Futuras Líneas de investigación}

A continuación se exponen las líneas que se piensan seguir a corto y mediano plazo a partir de este trabajo de Tesis.

Como se comenta en Cardenas et al. (2013) aproximadamente el $50 \%$ de los aerogeneradores instalados están equipados con generadores de rotor bobinado. En este sentido, es de interés el desarrollo de técnicas de control avanzadas que garanticen la estabilidad y la optimización de la generación de energía eléctrica basada en un recurso renovable. Específicamente, es de interés estudiar los problemas de variaciones de tensión de forma desbalanceada en el contexto de técnicas de control basadas en pasividad y en particular en moldeo de potencia.

Actualmente, en el campo de las micro redes y en el empleo de generación renovable existe un renovado interés en este campo de investigación en función de sus características. En cuanto a la micro red eléctrica analizada, en principio, los trabajos futuros involucrarán el diseño de controladores cuando la micro red este funcionando de forma aislada o vinculada a la red de distribución.

Adicionalmente se explorarán otras técnicas derivadas del empleo de sincrofasores (Wache 
y Murray, 2011) o utilizando observadores de estado a los efectos de asegurar tanto la estabilidad de la micro red conectada o desconectada de la red principal.

En todos los casos es necesario considerar las restricciones en las acciones de control, incorporando técnicas de acondicionamiento de referencia o equivalentes.

En este trabajo de tesis no se requirió la coordinación del control propuesto con ningún otro controlador de la red, como por ejemplo el control del generador sincrónico. Esto agregó un grado de flexibilidad en el diseño. No obstante, podría explorarse la posibilidad de obtener una única función de Lyapunov para toda la micro red considerada y, en función de la disponibilidad en las acciones de control o la importancia de la perturbación en la red eléctrica, establecer una estrategia de control que pueda definir acciones prioritarias o coordinadas en función de la necesidad. 


\section{BIBLIOGRAFÍA}

Ackermann, T. Wind power in power systems. John Wiley Sons, Hoboken (2012). ISBN 978-0470974162.

Anderson, P. M. Power system control and stability. IEEE Press Wiley-Interscience, Piscataway, N.J (2003). ISBN 978-0471238621.

Arraña, I., J. Chemes, F. Fernández, P. Frattin, F. Zelaschi, y N. Di Ruscio. Análisis del recurso eólico de la ciudad de rosario. En Jóvenes Investigadores Tecnológicos 2014 (2014).

Astolfi, A., R. Ortega, y R. Sepulchre. Stabilization and disturbance attenuation of nonlinear systems using dissipativity theory. European Journal of Control, 8(5):408 - 431 (2002). ISSN 0947-3580.

Barton, J. y D. Infield. Energy storage and its use with wind power. En Power Engineering Society General Meeting, 2005. IEEE, págs. 1934-1938 Vol. 2 (2005).

Batlle, C. y A. Dòria-Cerezo. De la mecánica clásica y las leyes de kirchhoff a los sistemas hamiltonianos con puertos. La Gaceta de la Real Sociedad Matemática Española, 13(1) (2010). ISSN 1138-8927.

Bianchi, F. D., H. De Battista, y R. J. Mantz. Wind turbine control systems: Principles, modelling and gain scheduling design. Advances in Industrial Control series. SpringerVerlag London Ltd., London (2006).

Bidram, A., F. Lewis, y A. Davoudi. Distributed control systems for small-scale power networks: Using multiagent cooperative control theory. IEEE Control Systems, 34(6):5677 (2014). ISSN 1066-033X.

Boicea, V. Energy storage technologies: The past and the present. Proceedings of the IEEE, 102(11):1777-1794 (2014). ISSN 0018-9219.

Brayton, R. K. y J. Moser. A theory of nonlinear networks i. Quaterly of applied mathematics, XXII(1):1-32 (1964a).

Brayton, R. K. y J. Moser. A theory of nonlinear networks, ii. Quaterly of applied mathematics, XXII(2):81 - 104 (1964b). 
Burton, T., N. Jenkins, D. Sharpe, y E. Bossanyi. Wind energy handbook. Wiley, Chichester, West Sussex (2011). ISBN 978-0-470-69975-1.

Bustos, G., L. Vargas, F. Milla, D. Saez, H. Zareipour, y A. Nunez. Comparison of fixed speed wind turbines models: A case study. En IECON 2012 - 38th Annual Conference on IEEE Industrial Electronics Society, págs. 961-966 (2012). ISSN 1553-572X.

Cardenas, R., R. Peña, S. Alepuz, y G. Asher. Overview of control systems for the operation of dfigs in wind energy applications. Industrial Electronics, IEEE Transactions on, 60(7):2776-2798 (2013). ISSN 0278-0046.

Chen, Z. y F. Blaabjerg. Wind farm - A power source in future power systems. Renewable and Sustainable Energy Reviews, 13(6-7):1288-1300 (2009). ISSN 1364-0321.

Chica Leal, A., C. Rodríguez, y F. Piedrahita. A proposal for microgrids control architecture as aggregator. En Innovative Smart Grid Technologies Latin America (ISGT LATAM), 2015 IEEE PES, págs. 473-478 (2015).

Chowdhury, S., S. Chowdhury, y P. Crossley. Microgrids and active distribution networks. Institution of Engineering and Technology, London (2009). ISBN 978-1849190145.

Di Marzio, G., J. Eek, J. Tande, y O. Fosso. Implication of grid code requirements on reactive power contribution and voltage control strategies for wind power integration. En Clean Electrical Power, 200\%. ICCEP '0\%. International Conference on, págs. 154-158 (2007).

Dirksz, D. y J. Scherpen. Power-based setpoint control: Experimental results on a planar manipulator. Control Systems Technology, IEEE Transactions on, 20(5):1384-1391 (2012a). ISSN 1063-6536.

Dirksz, D. A. y J. M. Scherpen. Power-based control: Canonical coordinate transformations, integral and adaptive control. Automatica, 48(6):1045 - 1056 (2012b). ISSN 0005-1098.

Dirksz, D. A. y J. M. A. Scherpen. Power-based adaptive and integral control of standard mechanical systems. En Decision and Control (CDC), 2010 49th IEEE Conference on, págs. 4612-4617 (2010). ISSN 0743-1546.

Dobson, I. y H.-D. Chiang. Towards a theory of voltage collapse in electric power systems. Systems \& Control Letters, 13(3):253 - 262 (1989). ISSN 0167-6911.

Donaire, A. y S. Junco. On the addition of integral action to port-controlled hamiltonian systems. Automatica, 45(8):1910 - 1916 (2009). ISSN 0005-1098.

Donaire, A., R. Mehra, R. Ortega, S. Sumeet, J. Romero, F. Kazi, y N. Singh. Shaping the energy of mechanical systems without solving partial differential equations. Automatic Control, IEEE Transactions on, $\operatorname{PP}(99): 1-1$ (2015). ISSN 0018-9286.

Espinar, B. y D. Mayer. The role of energy storage for mini-grid stabilization. Informe técnico, International energy agency. (2011).

Evangelista, C., F. Valenciaga, y P. Puleston. Multivariable 2-sliding mode control for a wind energy system based on a double fed induction generator. International Journal of Hydrogen Energy, 37(13):10070 - 10075 (2012). ISSN 0360-3199.

EWEA. Wind energy scenearios for 2020. Informe técnico, The European Wind Energy Association (2014). 
Ezzat, M., M. Benbouzid, S. Muyeen, y L. Harnefors. Low-voltage ride-through techniques for dfig-based wind turbines: state-of-the-art review and future trends. En Industrial Electronics Society, IECON 2013 - 39th Annual Conference of the IEEE, págs. 7681-7686 (2013). ISSN 1553-572X.

Farias, M., P. Battaiotto, y M. Cendoya. Investigation of upqc for sag compensation in wind farms to weak grid connections. En Industrial Technology (ICIT), 2010 IEEE International Conference on, págs. 937-942 (2010).

Favache, A. y D. Dochain. Power-shaping control of reaction systems: The CSTR case. Automatica, 46(11):1877 - 1883 (2010). ISSN 0005-1098.

Fernández, R., R. Mantz, y P. Battaiotto. Linear and non-linear control of wind farms. contribution to the grid stability. International Journal of Hydrogen Energy, 35(11):6019 - 6024 (2010). ISSN 0360-3199.

Fernández, R., F. Valenciaga, y R. Peña. Nonlinear control of a permanent magnet synchronous wind generator. En 2015 IEEE PES Innovative Smart Grid Technologies Latin America (ISGT LATAM) (2015 ISGT-LA), págs. 236-239. Montevideo, Uruguay (2015).

Galli, S., A. Scaglione, y Z. Wang. For the grid and through the grid: The role of power line communications in the smart grid. Proceedings of the IEEE, 99(6):998-1027 (2011). ISSN 0018-9219.

García-Canseco, E., D. Jeltsema, R. Ortega, y J. M. Scherpen. Power-based control of physical systems. Automatica, 46(1):127 - 132 (2010). ISSN 0005-1098.

García-Canseco, E., R. Ortega, J. M. A. Scherpen, y D. Jeltsema. Power shaping control of nonlinear systems: A benchmark example. Lecture notes in control and Information sciences, 366(366):135 - 146 (2007). ISSN 01708643.

Garelli, F., R. J. Mantz, y H. De Battista. Advanced control for constrained processes and systems. Institution of Engineering and Technology, Stevenage (2011). ISBN 9781849192620 .

Girsang, I., J. Dhupia, E. Muljadi, M. Singh, y L. Pao. Gearbox and drivetrain models to study dynamic effects of modern wind turbines. En Energy Conversion Congress and Exposition (ECCE), 2013 IEEE, págs. 874-881 (2013).

Grbovic, P. Ultracapacitors in power conversion systems : applications, analysis, and design from theory to practice. Wiley, Chichester, West Sussex, United Kingdom (2014). ISBN 978-1118356265.

Guerrero, J., J. Vasquez, J. Matas, L. de Vicuña, y M. Castilla. Hierarchical control of droopcontrolled ac and dc microgrids; a general approach toward standardization. Industrial Electronics, IEEE Transactions on, 58(1):158-172 (2011). ISSN 0278-0046.

GWEC. Global wind energy outlook. Informe técnico, Global Wind Energy Council Greenpeace (2014).

Hacha, E. Power electronic control in electrical systems.. Newnes (2002).

Haddad, W. y C. VijaySekhar. Nonlinear dynamical systems and control : a Lyapunov-based approach. Princeton University Press, Princeton (2008). ISBN 978-0691133294. 
Hamsic, N., A. Schmelter, A. Mohd, E. Ortjohann, E. Schultze, A. Tuckey, y J. Zimmermann. Increasing renewable energy penetration in isolated grids using a flywheel energy storage system. En Power Engineering, Energy and Electrical Drives, 200\%. POWERENG $200 \%$. International Conference on, págs. 195-200 (2007).

Hansen, A. D., P. Sórensen, F. Iov, y F. Blaabjerg. Centralised power control of wind farm with doubly fed induction generators. Renewable Energy, 31(7):935 - 951 (2006). ISSN 0960-1481.

Heier, S. Grid integration of wind energy : onshore and offshore conversion systems. Wiley, Chichester, West Sussex, United Kindgom (2014). ISBN 978-1119962946.

Hill, D. Nonlinear dynamic load models with recovery for voltage stability studies. Power Systems, IEEE Transactions on, 8(1):166-176 (1993). ISSN 0885-8950.

Hossain, E., E. Kabalci, R. Bayindir, y R. Perez. Microgrid testbeds around the world: State of art. Energy Conversion and Management, 86(0):132 - 153 (2014). ISSN 0196-8904.

Hou, J., Z. Xu, y Z. Y. Dong. Load modeling practice in a smart grid environment. En Electric Utility Deregulation and Restructuring and Power Technologies (DRPT), 2011 4th International Conference on, págs. 7-13 (2011).

Iov, F. B. Converter Application in Future European Electricity Network. Informe técnico, UNIFLEX-PM (2006).

Jeltsema, D. Modeling and Control of Nonlinear Networks: A Power-Based Perspective. Delft University of Technology (2005). ISBN 9789085590484.

Jeltsema, D., R. Ortega, y J. M. Scherpen. An energy-balancing perspective of interconnection and damping assignment control of nonlinear systems. Automatica, 40(9):1643 - 1646 (2004). ISSN 0005-1098.

Jeltsema, D. y J. M. A. Scherpen. A dual relation between port-hamiltonian systems and the Brayton-Moser equations for nonlinear switched RLC circuits. Automatica, 39(6):969 - 979 (2003). ISSN 0005-1098.

Jeltsema, D. y J. M. A. Scherpen. A power-based description of standard mechanical systems. Systems 83 Control Letters, 56(5):349 - 356 (2007). ISSN 0167-6911.

Jeltsema, D. y J. M. A. Scherpen. Dynamics and Control of Switched Electronic Systems: Advanced Perspectives for Modeling, Simulation and Control of Power Converters, capítulo Power-Based Modelling, págs. 245-271. Springer London, London (2012). ISBN 978-1-44712885-4.

Kaldellis, J. Stand-alone and hybrid wind energy systems, Technology, energy storage and applications. Woodhead Publishing Limited, Cambridge (2010). ISBN 9781845695279.

Karlsson, D. y D. Hill. Modelling and identification of nonlinear dynamic loads in power systems. Power Systems, IEEE Transactions on, 9(1):157-166 (1994). ISSN 0885-8950.

Katiraei, F., R. Iravani, N. Hatziargyriou, y A. Dimeas. Microgrids management. Power and Energy Magazine, IEEE, 6(3):54-65 (2008). ISSN 1540-7977.

Khan, R. H. y J. Y. Khan. Wide area pmu communication over a wimax network in the smart grid. En Smart Grid Communications (SmartGridComm), 2012 IEEE Third International Conference on, págs. 187-192 (2012). 
Kokotovic, P. Recent advances in nonlinear control. En Control, Communications and Signal Processing, 2004. First International Symposium on, págs. 721- (2004).

Kokotovic, P. y M. Arcak. Constructive nonlinear control: a historical perspective. Automatica, 37(5):637 - 662 (2001). ISSN 0005-1098.

Krause, P., O. Wasynczuk, y S. D. Sudhoff. Analysis of electric machinery. IEEE Press, New York (1995). ISBN 978-0780311015.

Kundur, P. Power Systems Stability and Control.. McGraw-Hill (1993).

Lasseter, R. y P. Paolo. Control and Design of Microgrid Components, Final project Report. Power Systems Engineering Research Center, Wisconsin-Madison (2006).

Ma, J., R. He, y D. Hill. Composite load modeling via measurement approach. En Power Engineering Society General Meeting, 2006. IEEE, págs. 1 pp.- (2006).

Martin, G. Wind turbines types, economics and development. Nova Science, New York (2010). ISBN 978-1-60741-849-8.

Martinez, M., G. Tapia, A. Susperregui, y H. Camblong. Sliding-mode control for dfig rotorand grid-side converters under unbalanced and harmonically distorted grid voltage. Energy Conversion, IEEE Transactions on, 27(2):328-339 (2012). ISSN 0885-8969.

Matevosyan, J., S. Martinez Villanueva, S. Djokic, J. Acosta, S. Mat Zali, F. Resende, y J. Milanovic. Aggregated models of wind-based generation and active distribution network cells for power system studies - literature overview. En PowerTech, 2011 IEEE Trondheim, págs. 1-8 (2011).

Miller, N., J. J. Sanchez-Gasca, W. Price, y R. Delmerico. Dynamic modeling of ge 1.5 and 3.6 MW wind turbine-generators for stability simulations. En Power Engineering Society General Meeting, 2003, IEEE, tomo 3, págs. 1977-1983 Vol. 3 (2003).

Mohseni, M., M. Masoum, y S. Islam. Emergency control of dfig-based wind turbines to meet new european grid code requirements. En Innovative Smart Grid Technologies (ISGT), 2011 IEEE PES, págs. 1-6 (2011).

Mokui, H., M. Masoum, y M. Mohseni. Review on australian grid codes for wind power integration in comparison with international standards. En Power Engineering Conference (AUPEC), 2014 Australasian Universities, págs. 1-6 (2014).

Montoya, M., R. Sherick, P. Haralson, R. Neal, y R. Yinger. Islands in the storm: Integrating microgrids into the larger grid. Power and Energy Magazine, IEEE, 11(4):33-39 (2013). ISSN 1540-7977.

Muhando, E., T. Senjyu, A. Uehara, y T. Funabashi. Gain-scheduled $\mathcal{H}_{\infty}$ control for wecs via lmi techniques and parametrically dependent feedback part I: Model development fundamentals. Industrial Electronics, IEEE Transactions on, 58(1):48-56 (2011). ISSN 0278-0046.

Olimpo, L., N. Jenkins, J. Ekanayake, P. Cartwright, y M. Hughes. Wind energy generation modelling and control. John Wiley Sons, Hoboken, NJ (2009). ISBN 9780470714331.

Ortega, R. Passivity-based control of euler-lagrange systems : mechanical, electrical and electromechanical applications. Springer-Verlag London Ltd, Londres (1998). ISBN 1852330163. 
Ortega, R., A. V. der Schaft, B. Maschke, y G. Escobar. Interconnection and damping assignment passivity-based control of port-controlled hamiltonian systems. Automatica, 38(4):585 - 596 (2002a). ISSN 0005-1098.

Ortega, R., G. Espinosa-Perez, y A. Astolfi. Theory for the user and application examples of the passivity-based control for ac electric machines. En Industrial Electronics (ISIE), 2012 IEEE International Symposium on, págs. 758-763 (2012). ISSN 2163-5137.

Ortega, R. y E. García-Canseco. Interconnection and damping assignment passivity-based control: A survey. European Journal of Control, 10(5):432 - 450 (2004). ISSN 0947-3580.

Ortega, R. y E. Garcia-Canseco. Interconnection and damping assignment passivity-based control: towards a constructive procedure - part i. En Decision and Control, 2004. CDC. 43rd IEEE Conference on, tomo 4, págs. 3412-3417 Vol.4 (2004). ISSN 0191-2216.

Ortega, R., D. Jeltsema, y J. M. A. Scherpen. Power shaping: a new paradigm for stabilization of nonlinear RLC circuits. Automatic Control, IEEE Transactions on, 48(10):1762-1767 (2003a). ISSN 0018-9286.

Ortega, R., D. Jeltsema, y J. M. A. Scherpen. Stabilization of nonlinear RLC circuits: power shaping and passivation. En Decision and Control, 2003. Proceedings. 42nd IEEE Conference on, tomo 6, págs. 5597-5602 Vol.6 (2003b). ISSN 0191-2216.

Ortega, R. y J. Romero. Robust integral control of port-hamiltonian systems: The case of non-passive outputs with unmatched disturbances. En Decision and Control and European Control Conference (CDC-ECC), 2011 50th IEEE Conference on, págs. 3222-3227 (2011). ISSN 0743-1546.

Ortega, R. y J. G. Romero. Robust integral control of port-hamiltonian systems: The case of non-passive outputs with unmatched disturbances. Systems \& Control Letters, 61(1):11 - 17 (2012). ISSN 0167-6911.

Ortega, R., M. Spong, F. Gomez-Estern, y G. Blankenstein. Stabilization of a class of underactuated mechanical systems via interconnection and damping assignment. Automatic Control, IEEE Transactions on, 47(8):1218-1233 (2002b). ISSN 0018-9286.

Ortega, R. y M. W. Spong. Adaptive motion control of rigid robots: A tutorial. Automatica, 25(6):877 - 888 (1989). ISSN 0005-1098.

Ortega, R., A. Van der Schaft, I. Mareels, y B. Maschke. Putting energy back in control. IEEE Control Systems, 21(2):18-33 (2001a). ISSN 1066-033X.

Ortega, R., A. J. van der Schaft, I. Mareels, y B. Maschke. Energy shaping control revisited, págs. 277-307. Springer London, London (2001b). ISBN 978-1-84628-570-7.

Palese, C., J. M. Gardiol, y J. L. Lassig. Mapas de velocidad media del viento en el centro-este de la provincia de neuquen. Meteorologica, 34(1):17-27 (2009).

Parhizi, S., H. Lotfi, A. Khodaei, y S. Bahramirad. State of the art in research on microgrids: A review. Access, IEEE, 3:890-925 (2015). ISSN 2169-3536.

Park, R. Two-reaction theory of synchronous machines generalized method of analysis-part i. American Institute of Electrical Engineers, Transactions of the, 48(3):716-727 (1929). ISSN 0096-3860. 
Peña, R., J. Clare, y G. Asher. Doubly fed induction generator using back-to-back pwm converters and its application to variable-speed wind-energy generation. Electric Power Applications, IEE Proceedings, 143(3):231-241 (1996). ISSN 1350-2352.

Peña, R., R. Fernández, y R. Mantz. Passivity control via power shaping of a wind turbine in a dispersed network. International Journal of Hydrogen Energy, 39(16):8846 - 8851 (2014). ISSN 0360-3199.

Peña, R., R. Fernández, R. Mantz, y P. Battaiotto. Power-based control with integral action for wind turbines connected to the grid. International Journal of Control, 88(10):2143-2153 (2015).

Peña, R., R. Fernández, R. Mantz, y P. Battaiotto. Control por moldeo de potencia de un aerogenerador en generación distribuida. En Reunión de Trabajo en Procesamiento de la Información y Control (RPIC 2013), págs. 1-6 (2013).

Petersson, A., L. Harnefors, y T. Thiringer. Comparison between stator-flux and grid-fluxoriented rotor current control of doubly-fed induction generators. En Power Electronics Specialists Conference, 2004. PESC 04. 2004 IEEE 35th Annual, tomo 1, págs. 482-486 Vol.1 (2004). ISSN 0275-9306.

Piagi, P. y R. Lasseter. Autonomous control of microgrids. En Power Engineering Society General Meeting, 2006. IEEE, págs. 8 pp.-(2006).

Piwko, R., N. Miller, R. Girad, J. MacDowell, K. Clark, y A. Murdoch. Generator fault tolerance and grid codes. Power and Energy Magazine, IEEE, 8(2):18-26 (2010). ISSN 1540-7977.

Qizhong, L., Y. Lan, y W. Guoxiang. Comparison of control strategy for double-fed induction generator (dfig). En Measuring Technology and Mechatronics Automation (ICMTMA), 2011 Third International Conference on, tomo 1, págs. 741-744 (2011).

Ribeiro, P., B. Johnson, M. Crow, A. Arsoy, y Y. Liu. Energy storage systems for advanced power applications. Proceedings of the IEEE, 89(12):1744-1756 (2001). ISSN 0018-9219.

Romero, J. G., A. Donaire, y R. Ortega. Robust energy shaping control of mechanical systems. Systems $\&$ Control Letters, 62(9):770 - 780 (2013). ISSN 0167-6911.

Salam, F., J. Marsden, y P. Varaiya. Arnold diffusion in the swing equations of a power system. Circuits and Systems, IEEE Transactions on, 31(8):673-688 (1984). ISSN 00984094.

Sastry, S. Nonlinear system : analysis, stability, and control. Springer, New York (1999). ISBN 978-0387985138.

Sauer, P. y M. Pai. Power system dynamics and stability. Prentice Hall, Upper Saddle River, N.J (1998). ISBN 0-13-678830-0.

Sepulchre, R., M. Jankovic, y P. V. Kokotovic. Constructive nonlinear control. Springer, London New York (1997). ISBN 978-1447112457.

Shanker, T. y R. Singh. Wind energy conversion system: A review. En Engineering and Systems (SCES), 2012 Students Conference on, págs. 1-6 (2012). 
Slootweg, J. y W. Kling. Aggregated modelling of wind parks in power system dynamics simulations. En Power Tech Conference Proceedings, 2003 IEEE Bologna, tomo 3, págs. 6 pp. Vol.3- (2003).

Song, J., E. Cotilla-Sanchez, y T. Brekken. Load modeling methodologies for cascading outage simulation considering power system stability. En Technologies for Sustainability (SusTech), 2013 1st IEEE Conference on, págs. 78-85 (2013).

Tande, J. O. G. Exploitation of wind-energy resources in proximity to weak electric grids. Applied Energy, 65(1-4):395 - 401 (2000). ISSN 0306-2619.

Tang, X., W. Deng, y Z. Qi. Research on micro-grid voltage stability control based on supercapacitor energy storage. En Electrical Machines and Systems, 2009. ICEMS 2009. International Conference on, págs. 1-6 (2009).

Tang, X., W. Deng, y Z. Qi. Investigation of the dynamic stability of microgrid. Power Systems, IEEE Transactions on, 29(2):698-706 (2014). ISSN 0885-8950.

Toccaceli, G., P. Battaiotto, M. Cendoya, y R. Peña. GDR in weak grid for desalination plant. system topology and control strategy. En Transmission and Distribution: Latin America Conference and Exposition (T D-LA), 2012 Sixth IEEE/PES, págs. 1-8 (2012a).

Toccaceli, G., R. Peña, M. Cendoya, y P. Battaiotto. Sistema de generación distribuida renovable con capacidad de operación en isla. En AADECA 2012, págs. 1-6 (2012b).

Toccaceli, G., R. Peña, M. Cendoya, y P. Battaiotto. Rdg system in remote areas with utility or local feed topology and operation strategy. Journal of Mechanics Engineering and Automation, 4(7):575-583 (2014). ISSN 2159-5275.

Trombotto, V. G. Complejo hidroeléctrico río grande central en caverna de acumulación por bombeo. Boletín Energético, 1(14):1-11 (2004). ISSN 1668-1525.

Tsili, M. y S. Papathanassiou. A review of grid code technical requirements for wind farms. Renewable Power Generation, IET, 3(3):308-332 (2009). ISSN 1752-1416.

Ustun, T. S., C. Ozansoy, y A. Zayegh. Recent developments in microgrids and example cases around the world-A review. Renewable and Sustainable Energy Reviews, 15(8):4030 - 4041 (2011). ISSN 1364-0321.

Van der Hoven, I. Power spectrum of horizontal wind speed in the frequency range from 0.0007 to 900 cycles per hour. Automatica, 14(2):160-164 (1957).

Van der Schaft, A. y D. Jeltsema. Port-Hamiltonian Systems Theory: An Introductory Overview. Foundations and Trends in Systems and Control, NOW Publishing Inc. (2014). ISBN 978-1-60198-786-0.

Vas, P. Sensorless Vector And Direct Torque Control. Oxford Science Publications (1998).

Wache, M. y D. C. Murray. Application of synchrophasor measurements for distribution networks. En 2011 IEEE Power and Energy Society General Meeting, págs. 1-4 (2011). ISSN 1932-5517.

Walve, K. Modelling of power system components at severe disturbances. CIGRE (1986).

Willems, J. Dissipative dynamical systems part i: General theory. Archive for Rational Mechanics and Analysis, 45(5):321-351 (1972). Cited By 1246. 
Wu, B., Y. Lang, N. Zargari, y S. Kouro. Power Conversion and Control of Wind Energy Systems.. Wiley-IEEE Press (2011). ISBN 978-0-470-59365-3. 